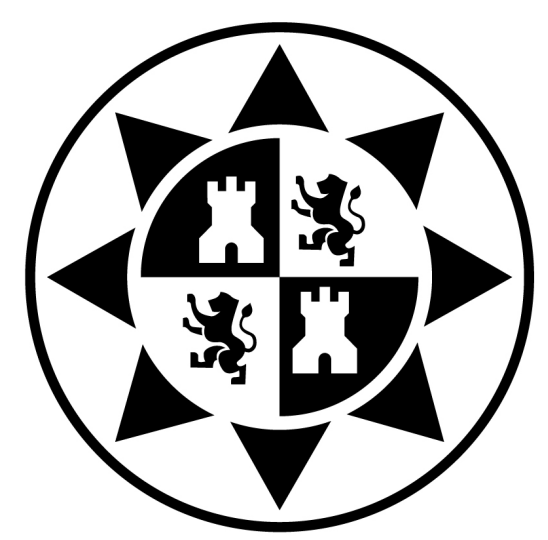

UNIVERSIDAD POLITÉCNICA DE CARTAGENA Unidad Predepartamental de Ingeniería Civil

\title{
ANÁLISIS DE LA VARIABILIDAD Y TENDENCIAS DE EVENTOS EXTREMOS DE PRECIPITACIÓN EN EL CONTEXTO DEL CAMBIO CLIMÁTICO: DESARROLLO DE UNA HERRAMIENTA DE SEGUIMIENTO DINÁMICO DE INUNDACIONES
}

Juan Diego Giraldo Osorio 



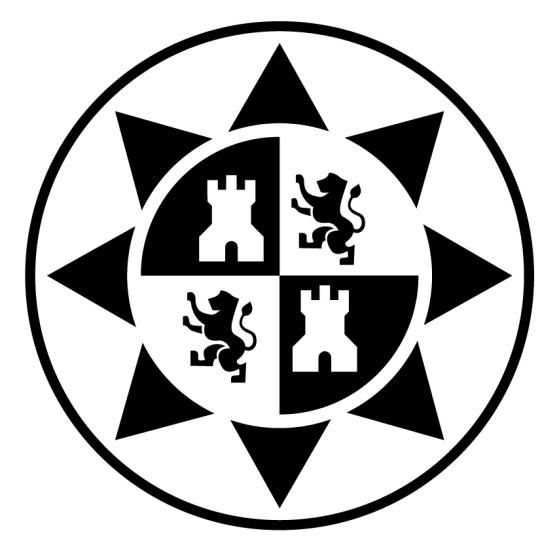

UNIVERSIDAD POLITÉCNICA DE CARTAGENA Unidad Predepartamental de Ingeniería Civil

\section{ANÁLISIS DE LA VARIABILIDAD Y TENDENCIAS DE EVENTOS EXTREMOS DE PRECIPITACIÓN} EN EL CONTEXTO DEL CAMBIO CLIMÁTICO: DESARROLLO DE UNA HERRAMIENTA DE SEGUIMIENTO DINÁMICO DE INUNDACIONES

Memoria presentada por Juan Diego Giraldo Osorio para optar al grado de Doctor por la Universidad Politécnica de Cartagena.

Dirigida por la Dra. Ingeniera de Caminos, Canales y Puertos Sandra G. García Galiano 

Esta memoria se presenta en la modalidad de compendio de publicaciones. Los artículos y registros de propiedad intelectual que constituyen la Tesis son los siguientes:

Artículo 1: García Galiano, S. G. and Giraldo Osorio, J. D., 2010. Analysis of impacts on hydrometeorological extremes in the Senegal river basin from REMO RCM. Meteorologische Zeitschrift, 19 (4), 375-384. doi 10.1127/0941-2948/2010/0457.

Artículo 2: Karambiri, H., García Galiano, S. G., Giraldo, J. D., Yacouba, H., Ibrahim, B., Barbier, B. Polcher, J., 2011. Assessing the impact of climate variability and climate change on runoff in West Africa: the case of Senegal and Nakambe River basins, Atmospheric Science Letters. AMMA Special Issue, 12: 109-115. doi: 10.1002/asl.317.

Artículo 3: Giraldo Osorio, J. D. and García Galiano, S. G., 2011. Building hazard maps of extreme daily rainy events from PDF ensemble, via REA method, on Senegal River Basin. Hydrology Earth System Science, 15, 3605-3615. doi: 10.5194/hess-153605-2011.

Artículo 4: Giraldo Osorio, J. D, and García Galiano, S. G., 2012. Development of a sub-pixel analysis method applied to dynamic monitoring of floods, International Journal of Remote Sensing, 33 (7), 2277-2295.

http://dx.doi.org/10.1080/01431161.2011.608091.

Registro de propiedad intelectual 1: Giraldo Osorio, J. D. y García Galiano, S. G., 2011. Subpixels Toolbox. Técnica de downscaling de imágenes satélite. Seguimiento dinámico de avenidas en grandes extensiones. Comandos GRASS desarrollados: i.subpixel, i.dod, i.isolate. UPCT RPI 08/2011/173.

Registro de propiedad intelectual 2: Giraldo Osorio, J. D. y García Galiano, S. G., 2011. Spells Toolbox. Procesamiento de series temporales de mapas ráster para la estimación de estadísticos de rachas. Extracción de las series temporales puntuales de rachas. Comandos GRASS desarrollados: r.series.spell, r.series.spell.point. UPCT RPI 08/2011/176.

Registro de propiedad intelectual 3: Giraldo Osorio, J. D. y García Galiano, S. G., 2011. Series Toolbox. Procesamiento de series temporales de mapas ráster para la estimación de estadísticos y extracción de series temporales puntuales. Comandos GRASS desarrollados: r.series.new, r.series.point. UPCT RPI 08/2011/177.

Capítulo de Libro Internacional: Garcia Galiano, S. G., Giraldo Osorio, J. D., 2011. Non stationary analysis of spatial patterns of extreme rainfall events in West Africa, In: Hydro-climatology: variability and change (Eds. S.W. Franks, E. Boegh, E. Blyth, D.M. Hannah, K.K. Yilmaz). IAHS-AISH Publication, 344, 75-81. IAHS Press, Wallingford, Oxfordshire, United Kingdom. ISBN 978-1-907161-19-3. ISSN 0144-7815. 



\section{Universidad}

Politécnica

de Cartagena

DT-12

\section{CONFORMIDAD DE SOLICITUD DEAUTORIZACIÓN DE DEPÓSITO DE} TESIS DOCTORAL POR EL/LA DIRECTOR/A DE LA TESIS

Da. Sandra G. García Galiano, Director/a de la Tesis doctoral "Análisis de la variabilidad y tendencias de eventos extremos de precipitación en el contexto del cambio climático: desarrollo de una herramienta de seguimiento dinámico de inundaciones".

\section{INFORMA:}

Que la referida Tesis Doctoral, ha sido realizada por D. Juan Diego Giraldo Osorio, dando mi conformidad para que sea presentada ante la Comisión de Doctorado.

La rama de conocimiento por la que esta tesis ha sido desarrollada es:

Ciencias

Ciencias Sociales y Jurídicas

$X$ Ingeniería y Arquitectura

En Cartagena, a 12 de Diciembre de 2011.

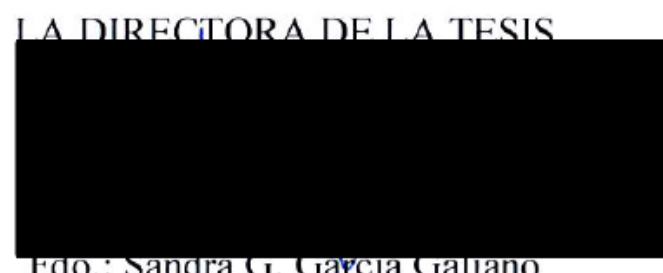

rao.: Sanara G. Garcla Galıano 



\title{
Universidad Politécnica de Cartagena
}

\section{CONFORMIDAD DE DEPOSITO DE TESIS DOCTORAL POR LA COMISIÓN ACADÉMICA DEL PROGRAMA}

\author{
D. Victoriano Martínez Álvarez, Presidente Delegado de la Comisión Académica del \\ Programa Gestión de Recursos Hídricos.
}

Da . Sandra G. García Galiano, Profesora Titular de Escuela Universitaria en la Unidad

Predepartamental de Ingeniería Civil.

\section{INFORMAN:}

Que la Tesis Doctoral titulada, "Análisis de la variabilidad y tendencias de eventos extremos de precipitación en el contexto del cambio climático: desarrollo de una herramienta de seguimiento dinámico de inundaciones", ha sido realizada por D. Juan Diego Giraldo Osorio, bajo la dirección y supervisión de la Dra. Sandra G. García Galiano, dando su conformidad a la misma la Comisión Académica, con la finalidad de que sea presentada ante la Comisión de Doctorado. Los siguientes artículos y registros de propiedad intelectual serán incluidos en la Tesis Doctoral, que será presentada mediante la alternativa de compendio de publicaciones:

- Artículo 1: García Galiano, S. G. and Giraldo Osorio, J. D., 2010. Analysis of impacts on hydrometeorological extremes in the Senegal river basin from REMO RCM. The 2nd Lund Regional-scale Climate Modelling Workshop: Part II. Meteorologische Zeitschrift, 19 (4), 375-384.

- Artículo 2: Karambiri, H., García Galiano, S. G., Giraldo, J. D., Yacouba, H., Ibrahim, B., Barbier, B. Polcher, J., 2011. Assessing the impact of climate variability and climate change on runoff in West Africa: the case of Senegal and Nakambe River basins, Atmospheric Science Letters. AMMA Special Issue, 12: 109115.

- Artículo 3: Giraldo Osorio, J. D. and García Galiano, S. G., 2011. Building hazard maps of extreme daily rainy events from PDF ensemble, via REA method, on Senegal River Basin. Hydrol. Earth Syst. Sci., 15, 3605-3615.

- Artículo 4: Giraldo Osorio, J. D, García Galiano, S. G., 2012. Development of a sub-pixel analysis method applied to dynamic monitoring of floods, International Journal of Remote Sensing, 33 (7), 2277-2295. Revista con clasificación A1 Colciencias para el año 2010.

- Registro de propiedad intelectual 1: Giraldo Osorio, J. D. y García Galiano, S. G., 2011. Subpixels Toolbox. Técnica de downscaling de imágenes satélite. Seguimiento dinámico de avenidas en grandes extensiones. Comandos GRASS desarrollados: i.subpixel, i.dod, i. isolate. UPCT RPI 08/2011/173.

- Registro de propiedad intelectual 2: Giraldo Osorio, J. D. y García Galiano, S. G., 2011. Spells Toolbox. Procesamiento de series temporales de mapas ráster para la estimación de los estadísticos de rachas. Extracción de las series temporales puntuales de rachas. Comandos GRASS desarrollados: r.series.spell, r.series.spell.point. UPCT RPI 08/2011/176.

- Registro de propiedad intelectual 3: Giraldo Osorio, J. D. y García Galiano, S. G., 2011. Series Toolbox. Procesamiento de series temporales de mapas ráster para la estimación de estadísticos y extracción de series temporales puntuales. Comandos GRASS desarrollados: r.series.new, r.series.point. UPCT RPI 08/2011/177.

- Capítulo de Libro Internacional: Garcia Galiano, S. G., Giraldo Osorio, J. D., 2011. Non stationary analysis of spatial patterns of extreme rainfall events in West Africa. In: Hydro-climatology: variability and change. IAHS Publ. 344, IAHS Press, Wallingford, Oxfordshire, United Kingdom.

Los artículos y los registros de propiedad intelectual, no serán presentados como parte de otra tesis doctoral en la modalidad de compendio de publicaciones. En todos los citados artículos el doctorando ha realizado una aportación relevante. 

Universidad

Politécnica

de Cartagena

\section{DT-13}

La Rama de conocimiento por la que esta tesis ha sido desarrollada es:

$\square$ Ciencias

$\square$ Ciencias Sociales y Jurídicas

$\mathrm{X}$ Ingeniería y Arquitectura

En Cartagena, a 05 de Diciembre de 2011.

EL PRESIDENTE DELEGADO

DE LA COMISIÓN ACADÉMICA

DEL PROGRAMA
LA DIRECTORA DE LA TESIS

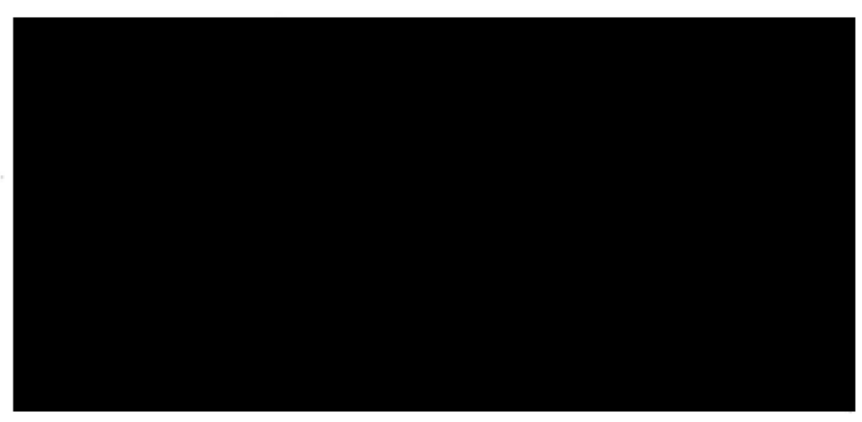

Fdo: D ${ }^{\mathrm{a}}$. Sandra G. García Galiano.

Fdo: D. Victoriano Martínez Álvarez. 



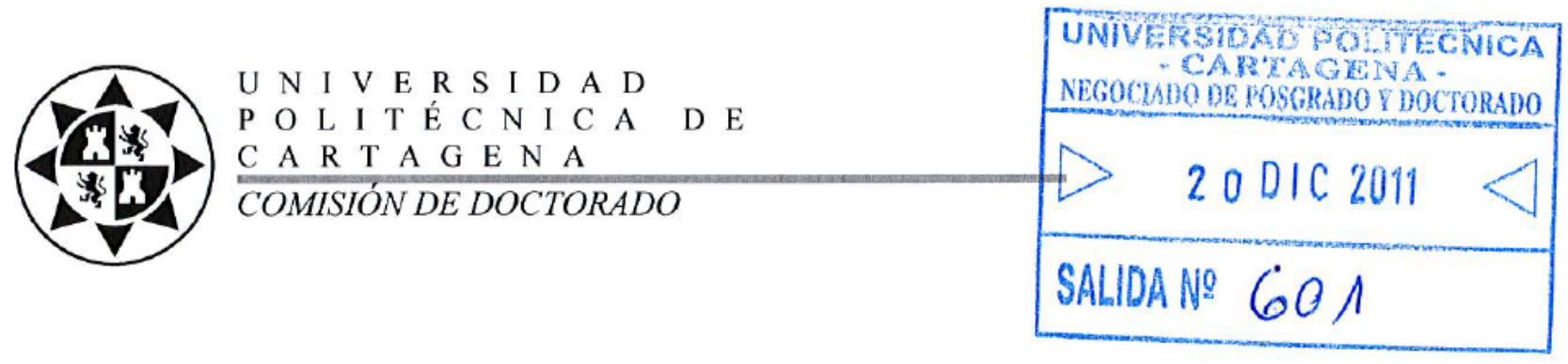

\section{Juan Diego Giraldo Osorio}

Vistos los informes favorables de los Directores de Tesis y el $\mathrm{V}^{\circ} \mathrm{B}^{\circ}$ de la Comisión Académica para la presentación de la Tesis Doctoral en la modalidad de "compendio de publicaciones" solicitada por D. Juan Diego Giraldo Osorio, la Comisión de Doctorado de la Universidad Politécnica de Cartagena, en reunión celebrada el 19 de diciembre de 2011, considerando lo dispuesto en el artículo 33 del Reglamento de Estudios Oficiales de Máster y Doctorado de la UPCT, aprobado en Consejo de Gobierno de 13 de abril de 2011,

\section{ACUERDA}

Autorizar la presentación de la Tesis Doctoral a D. Juan Diego Giraldo Osorio en la modalidad de compendio de publicaciones.

Contra el presente acuerdo, que no agota la vía administrativa, podrá formular recurso de alzada ante el Sr. Rector-Magnífico de la Universidad Politécnica de Cartagena, en el plazo de un mes a partir de la notificación de la presente.

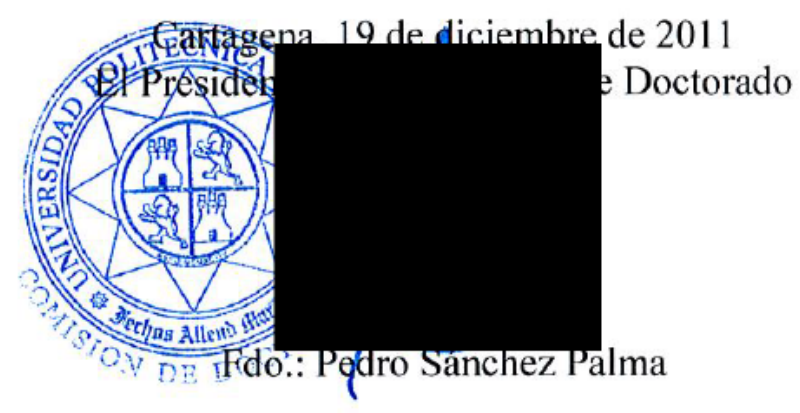





\section{AGRADECIMIENTOS}

Se agradece especialmente el apoyo recibido dentro del Proyecto AMMA FP6-004089 "African Monsoon Multidisciplinary Analysis". Basado en una iniciativa francesa, AMMA está constituido por un grupo científico multidisciplinario de carácter internacional. AMMA está financiado por un gran número de organismos, especialmente de Francia, Reino Unido, Estados Unidos de América y del Continente Africano. El Proyecto AMMA ha sido beneficiario de la mayor aportación económica dentro del Sexto Programa Marco de Investigación de la Comunidad Europea. Información detallada sobre la coordinación científica y los recursos financieros están disponibles en la página electrónica de AMMA Internacional http://www.amma-international.org.

Se agradece a la Secretaría de Estado de Investigación del Ministerio de Ciencia e Innovación de España, por el apoyo y financiación recibidos dentro del Proyecto I+D CGL2008-02530/BTE EVISA “Evaluación del Impacto de Sequías y del Uso del Suelo en el Ciclo Hidrológico mediante Asimilación de Datos de Teledetección en Modelos Hidrológicos".

Asimismo, se aprecia el soporte recibido desde la Confederación Hidrográfica del Segura (Ministerio de Medio Ambiente, Medio Rural y Marino), en el marco del Convenio de I+D denominado "Desarrollo de indicadores de sequía y escasez hídrica en el contexto del cambio climático: Apoyo al Mandato Water Scarcity and Droughts".

A mi Directora de Tesis Dra. Sandra G. García Galiano, Investigadora principal del Grupo de I+D Gestión de Recursos Hídricos de la UPCT. Muchas gracias por darme la oportunidad de crecer profesionalmente. Su decidido apoyo, su actitud abierta a la discusión, sus continuos consejos y acertados apuntes para refinar el trabajo y, especialmente, su paciencia fueron fundamentales para el éxito de la investigación.

A tantas personas maravillosas con las que me he topado durante estos seis años. La amistad y el apoyo que me brindaron son un valiosísimo recuerdo. 



\section{TABLA DE CONTENIDO}

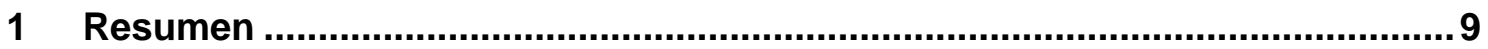

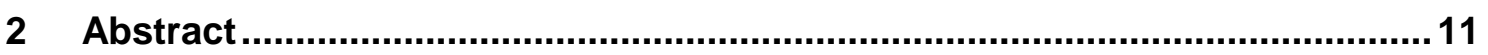

3 Motivación y Objetivos......................................................................................13

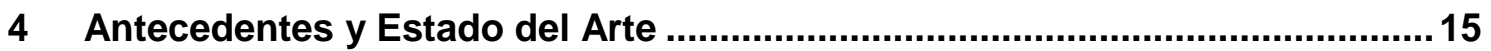

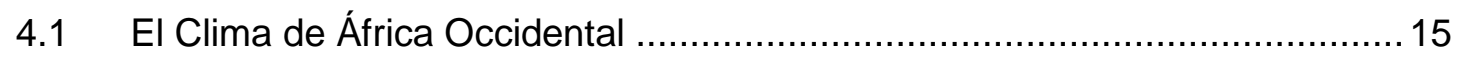

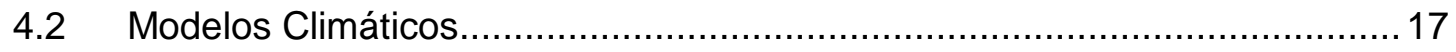

4.2.1 Modelos de circulación general ....................................................... 17

4.2.2 Modelos climáticos regionales para África Occidental ..........................20

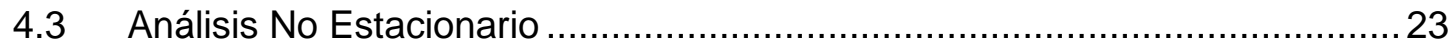

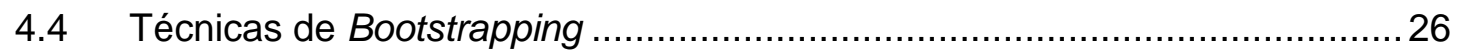

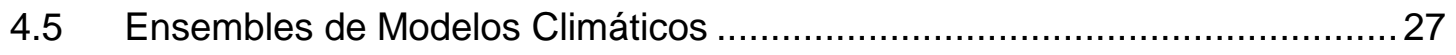

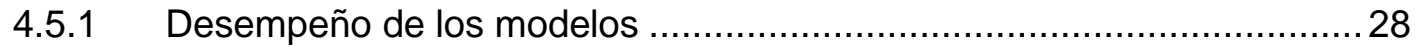

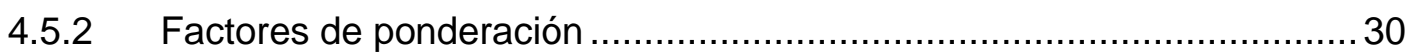

4.5.3 Métricas utilizadas para calcular los factores de ponderación..................31

4.6 Riesgo de Eventos Extremos................................................................... 32

4.7 Teledetección Aplicada a la Detección de Inundaciones.................................33

5 Publicaciones que Constituyen la Tesis .........................................................37

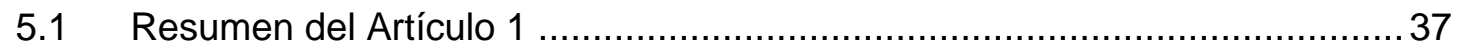

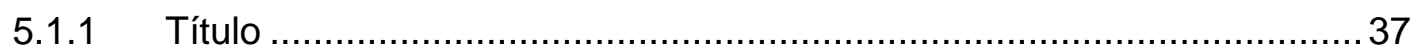

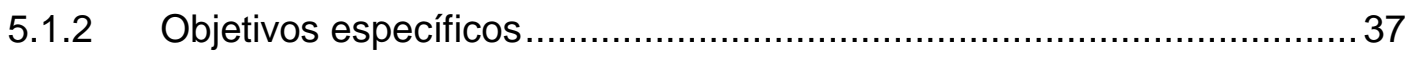

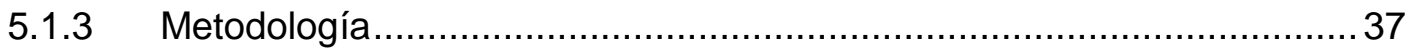

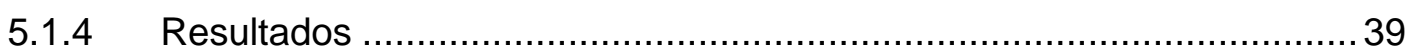

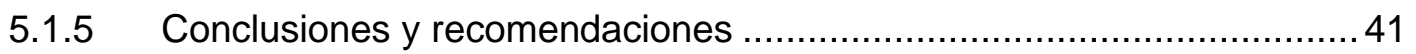

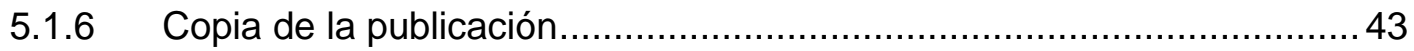

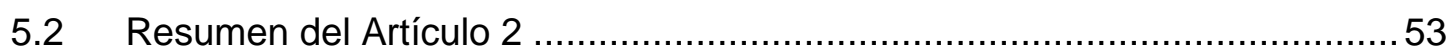

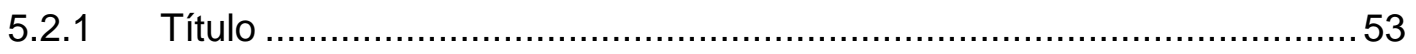

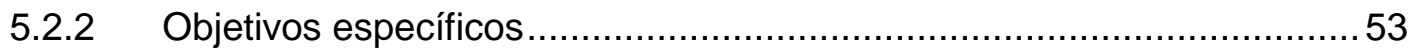

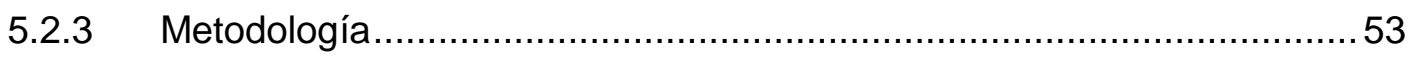

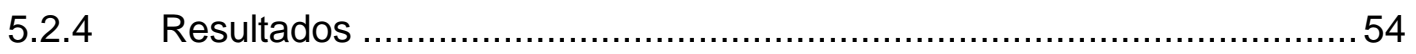

5.2.5 Conclusiones y recomendaciones …………………...................... 56 


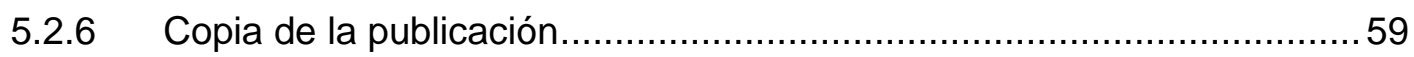

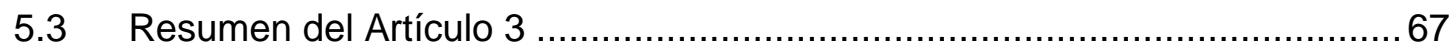

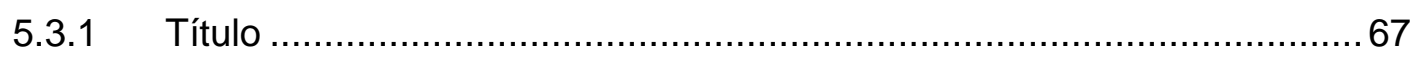

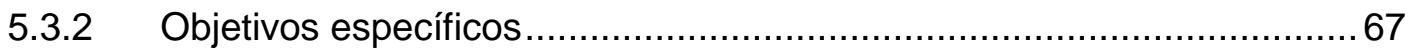

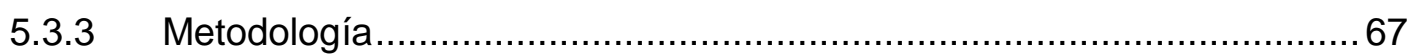

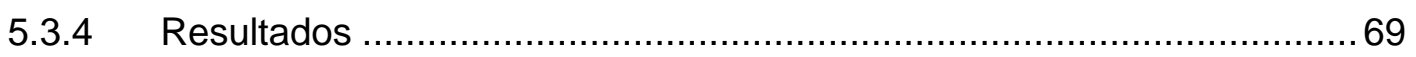

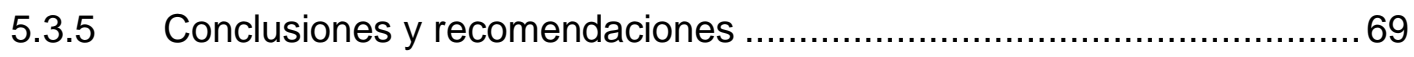

5.3.6 Copia de la publicación.............................................................. 71

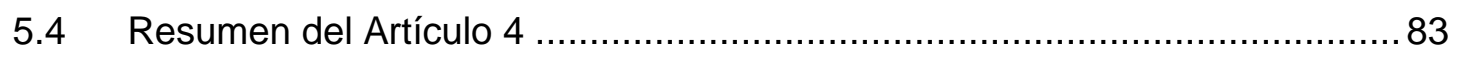

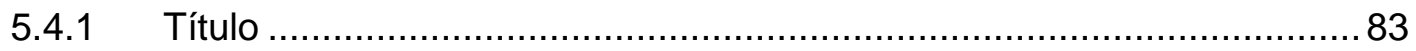

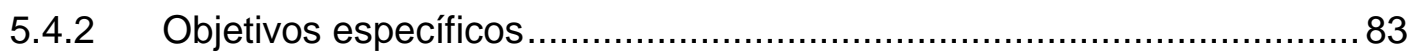

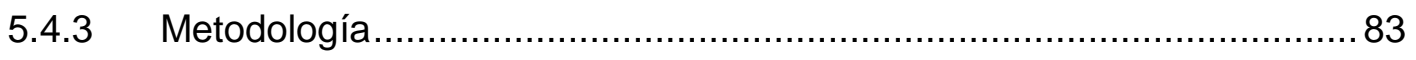

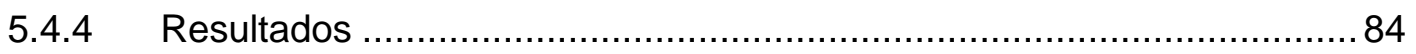

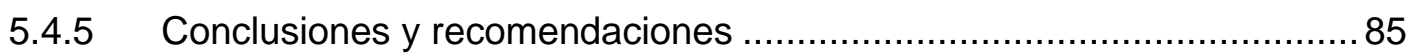

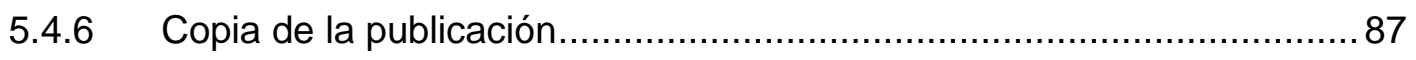

5.5 Resumen del Capítulo de Libro Internacional ........................................... 107

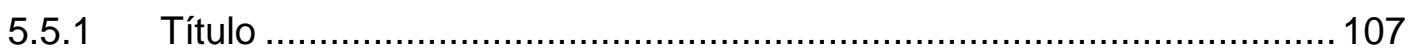

5.5.2 Objetivos específicos ......................................................... 107

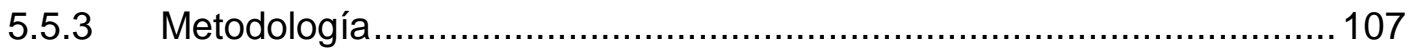

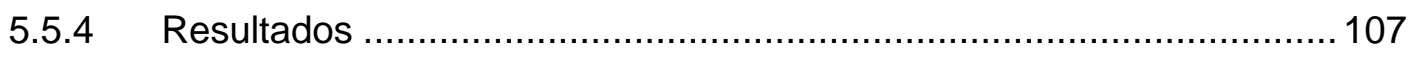

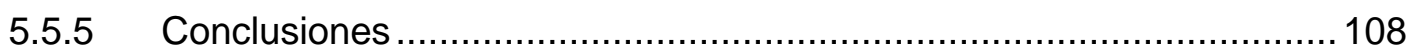

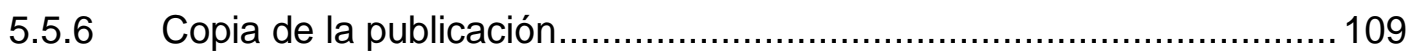

6 Registros de Propiedad Intelectual ............................................................117

6.1 Registro de Propiedad Intelectual 1 (Subpixels Toolbox) .......................... 117

6.1.1 Aportación al trabajo realizado ..................................................... 117

6.1.2 Copia del registro ............................................................... 119

6.2 Registro de Propiedad Intelectual 2 (Spells Toolbox)................................ 121

6.2.1 Aportación al trabajo realizado …................................................... 121

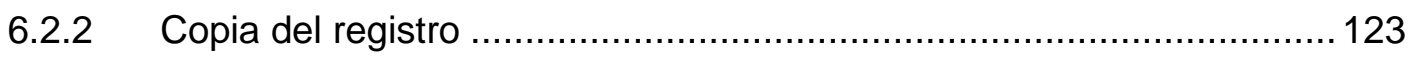

6.3 Registro de Propiedad Intelectual 3 (Series Toolbox) ............................... 125

6.3.1 Aportación al trabajo realizado ...................................................... 125

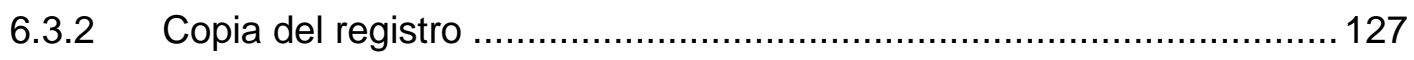


$7 \quad$ Resumen de los Resultados y Conclusiones ..................................................129

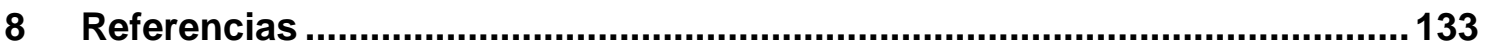

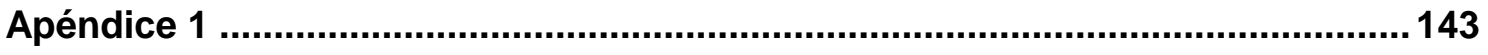

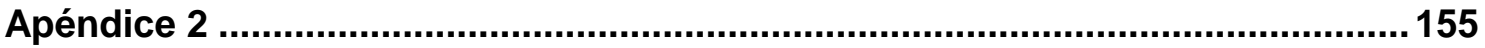

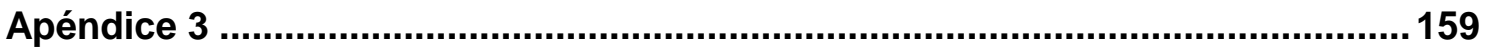

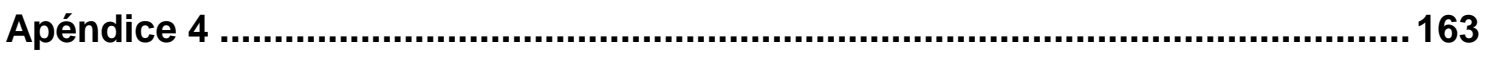





\section{LISTA DE FIGURAS}

Figura 1. Zona de estudio: (a) Precipitación media anual ( $\mathrm{mm}$ ) en África Occidental en el periodo 1961-1990 (base de datos CRU). (b) Ecorregiones al interior de la Cuenca del Río Senegal.

Figura 2. Representación esquemática de un GCM. El planeta es dividido en una malla esférica tridimensional. En cada celda de la malla se ejecutan diferentes proceso físicos del modelo. 18

Figura 3. África Occidental. (a) Dominio espacial de los RCM construidos para la región dentro del experimento RT3 del Proyecto ENSEMBLES. (b) Ubicación de la Cuenca del Río Senegal. 21

Figura 4. Ejemplo de análisis GAMLSS a la serie de AMDR del sitio 16 para el GKSS/CLM RCM. (a) Serie temporal de AMDR para el RCM (gris) y para los datos IRD (negro), en conjunto con las curvas de los centiles (5 al 95\%, en línea discontinua) que representan la distribución de probabilidad GAMLSS ajustada; (b) worm-plot y (c) qq-plot de los residuales. Se presentan además los estadísticos de los residuales. Se rechaza la hipótesis de normalidad si el coeficiente de Filliben es menor que 0.984 (Material suplementario en la discusión abierta del artículo Giraldo Osorio and García, 2011).

Figura 5. Diagrama de flujo para construir las PDF ensemble de las AMDR en cada sitio de análisis. Los diferentes procedimientos son: (a) lectura de los datos, (b) análisis del desvío, (c) análisis de convergencia a futuro, (d) cálculo REA, (e) análisis no estacionario, y (f) construcción de la PDF ensemble.

Figura 6. Métrica definida por Perkins et al. (2007). El $S_{S C O R E}$ es la medida del área bajo ambas curvas $f_{1}(x)$ y $f_{2}(x)$ (área sombreada). 32

Figura 7. Valor de la significancia de la prueba de hipótesis para la igualdad de la media de la precipitación anual (calculada con CRU y REMO) en el periodo 19611990 para África Occidental. (a) REMO-901, (b) REMO-902 y (c) REMO-903..... 40

Figura 8. Análisis GAMLSS de las AMDSL. (a) SAS en la Cuenca del Senegal, (b) WSS en la Cuenca del Senegal y (c) Cuenca del Nakambé. Las curvas del los centiles (5-95\%) están representadas por líneas discontinuas. .55

Figura 9. Diagrama de flujo de la metodología de análisis de sub-píxeles desarrollada para la detección de inundaciones. Se destacan los comandos GRASS desarrollados (tomado de Giraldo Osorio and García Galiano, 2012)..... 85 



\section{LISTA DE TABLAS}

Tabla 1. Familias de escenarios de GHGE del SRES. Se presenta el rango

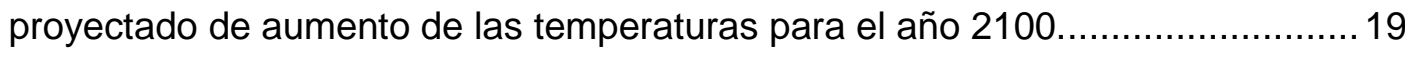

Tabla 2. Resumen de los RCMs utilizados, junto con las características de las bases de datos observados IRD y CRU........................................................... 22 



\section{RESUMEN}

La región Sudano Saheliana en África Occidental, una de las más pobres del planeta, se caracteriza por una gran variabilidad de las precipitaciones y un rápido crecimiento demográfico. Dada su vulnerabilidad climática, en la región los eventos extremos (sequías e inundaciones), causan cuantiosos daños que con frecuencia involucran perjuicios a la propiedad y pérdida de vidas humanas.

El conocimiento de las tendencias plausibles de los eventos extremos de precipitación es crucial desde una perspectiva política, para la administración de los recursos hídricos a escala de cuenca. Los análisis basados en datos observados y obtenidos desde Modelos Climáticos Regionales (Regional Climate Models -RCM-), han revelado un comportamiento no estacionario de las variables hidrometeorológicas. Los análisis de frecuencia tradicionales son estacionarios, pero se han detectado tendencias en las series históricas que obligan a utilizar modelos estadísticos no estacionarios para ajustar los parámetros de la Función de Densidad Probabilidad (Probability Density Function, PDF). Además, las proyecciones de cambio en los valores de los eventos extremos han mostrado gran divergencia entre los diferentes RCMs, incrementando la incertidumbre en las predicciones.

En este contexto, metodologías que consideren tanto la variabilidad proporcionada por cada RCM, como la naturaleza no estacionaria de las series temporales, deberían ser aplicadas para la construcción de mapas de riesgo de eventos hidrometeorológicos extremos. En la presente Tesis, se ha modelado la naturaleza no estacionaria de las series mediante las herramientas GAMLSS (Generalized Additive Models for Location, Scale and Shape), que permiten ajustar con mucha flexibilidad los parámetros de las PDF seleccionadas. Para enfrentar el problema de la incertidumbre generada por la divergencia en las predicciones, se ha optado por construir PDF ensemble utilizando técnicas de bootstrapping. Si bien, en los primeros trabajos presentados no se construyeron ensembles puesto que se disponía de muy pocos RCMs. Cuando el Proyecto Europeo ENSEMBLES hizo disponible a la comunidad científica todos los RCMs considerados para el dominio espacial África Occidental, se justificó la construcción de los ensembles multimodelo. Desde entonces, el trabajo de Tesis se ha centrado en la identificación y desarrollo de metodologías robustas y reproducibles de construcción de PDF ensemble de aplicación regional. El aporte de cada RCM para la construcción de las PDF ensemble se ha estimado de acuerdo con el método REA (Reliability Ensemble Average). Los factores REA se calcularon de acuerdo con una medida cuantitativa simple que mide el acuerdo entre la distribución de referencia 
(observada desde los datos históricos, o estimada para el futuro como una combinación de los RCMs), y las distribuciones provistas desde los datos de los RCMs. Se ha trabajado en la Cuenca del Río Senegal, considerando como variables la Longitud Máxima Anual de las Rachas Secas (Annual Maximum Dry Spell Length, AMDSL), y la Precipitación Diaria Máxima Anual (Annual Maximum Daily Rainfall, AMDR), con el objetivo de generar mapas de riesgo y evaluar impactos futuros.

El análisis regional de las AMDSL, considerando las ecorregiones al interior de la cuenca, ha predicho aumentos en su media y desviación estándar. Para las AMDR se construyeron mapas interpolados, desde estadísticos derivados de la PDF ensemble para cada sitio de análisis, junto con los intervalos de confianza. La diferencia calculada para mapas construidos en dos años de referencia (1990 como año de referencia del cambio, y 2050 como horizonte de predicción), reveló las tendencias espacio-temporales de las diferentes variables estudiadas. La distribución espacial de las tendencias de las AMDR demuestra que las precipitaciones extremas aumentarán significativamente en el Valle del Río Senegal debido al incremento en la media y la desviación estándar en esta región. Las conclusiones del trabajo, especialmente las distribuciones espaciales, constituyen verdaderos aportes a los procesos de toma de decisión, y al desarrollo de estrategias de mitigación y adaptación, para enfrentar el aumento de recurrencia de los eventos extremos debido al cambio climático.

Concretamente en el Valle del Río Senegal se ha observado una clara tendencia a aumentar de las precipitaciones máximas diarias para el horizonte 2050, en una zona especialmente sensible a las inundaciones debido a la alta densidad demográfica y su dependencia de la agricultura de recesión. Por ello, como medida de adaptación al cambio climático, se considera el seguimiento y alerta de inundaciones basadas en teledetección. Esta alternativa es aún más atractiva cuando los medios para realizar mediciones en tierra son escasos y contienen información poco confiable, como en el caso del Valle del Río Senegal. En este sentido, se desarrolló una herramienta computacional para el seguimiento dinámico de inundaciones utilizando imágenes de satélite. Ello requiere la utilización de imágenes con alta resolución temporal, con el inconveniente de que tales imágenes usualmente presentan resoluciones espaciales bajas. Con el fin de aumentar la resolución espacial, se ha desarrollado una metodología innovadora de Análisis de Sub-píxeles (Sub-pixels Analysis, SA) que, considera la topografía digital junto con algunos de sus atributos derivados. Aplicando esta herramienta para el seguimiento de un evento de inundación en el Valle del Río Senegal, se ha obtenido una mejora sustancial en la delimitación de las áreas inundadas con el SA, comparándolo con un método de clasificación supervisado tradicional que ha utilizado las mismas imágenes de satélite. 


\section{ABSTRACT}

The Sudano Sahelian region in West Africa, one of the poorest in the World, is characterized by the great variability of rainfall and the rapid population growth. Given its climate vulnerability, extreme events of drought and rainfall cause extensive damage, which often involve property damage and human lives losses.

The knowledge about plausible trends of extreme events is crucial from a policy perspective, for water resources management at basin level. Analyses based on observed data and obtained from Regional Climate Model ( $R C M)$ have revealed a nonstationary behaviour of hydrometeorological variables. The traditional frequency analysis suppose stationarity. Nevertheless, non-stationarity probabilistic models should be used to adjust the Probability Density Function (PDF) parameters, because have been detected significant trends in the historical data. Also, change projections of extreme events have shown great divergence between RCMs, increasing the forecast uncertainty.

In this context, methodologies should be applied to take into account the variability provided by RCMs, as well as non-stationary nature of time series in order to draw risk maps of extreme events (drought and extreme rainfall). The GAMLSS tool (Generalized Additive Models for Location, Scale and Shape) has been chosen to model non-stationary nature of time series obtained from RCMs. GAMLSS is flexible to adjust the parameters of the selected PDF. It was decided to build PDF ensemble using bootstrapping techniques, to face the uncertainty problem generated by the models forecast divergence. The early papers presented in the compendium, ensembles were not built because a small number of models were available at that time. However, releasing to the scientific community all of the RCMs which were considered for Africa domain, by the ENSEMBLE European Project, the construction of ensembles was justified. Since then, the Thesis has been focused on identifying and developing robust and reproducible methods to build PDF ensemble of regional application. The weight of each RCM in the PDF ensemble was estimated via REA method (Reliability Ensemble Average). The REA factors were calculated according to a simple quantitative metric which measures the "distributional agreement" between the reference distribution (from historical data, or estimated for the future as a combination of RCMs), and distributions from data provided by RCMs. The Senegal River Basin has been the study zone. The Annual Maximum Dry Spell Length (AMDSL) and the Annual Maximum Daily Rainfall (AMDR) have been considered as work variables, with the objective of drawing risk maps and evaluating future impacts. 
The AMDSL were regionally analyzed, taking into account the identified ecoregions within the basin. The analysis has shown that, in general, the models predict increases in mean and standard deviation of the AMDSL. Interpolated maps were built from statistics derived from the PDF ensemble at each grid-site, together with confidence intervals for the AMDR. The difference between the maps for two reference years (1990 as reference year of change, and 2050 as a plausible prediction horizon) was assessed, and spatiotemporal trends of the different variables studied were obtained. The spatial distribution of trends shows that extreme precipitation will increase significantly in the Senegal River Valley, due to the increase of the AMDR mean and standard deviation at the region. The outcomes of the work, specially the maps with the spatial distributions, constitute real contributions to decision making processes, and to develop mitigation and adaptation strategies to cope with extreme events associated with climate change.

In the Senegal River Valley, the results have shown a clear rising trend of maximum daily rainfall for the forecast horizon 2050. The Senegal River Valley is particularly sensitive to floods, due to the large number of people who are settled on the riverside area and depend on recession agriculture. Therefore, as an adaptation measure to climate change, the flood monitoring and warning based on remote sensing has been considered. The monitoring of natural phenomena with remote sensing in extensive areas is a reliable and economical alternative. It is even more attractive when the means to conduct ground-based measurements are sparse and contain unreliable information, such as the Senegal River Valley. In this sense, a computational tool for floods detection, using satellite imagery was developed. However, the flood monitoring requires using satellite images with high temporal resolution due to its highly dynamic nature, with the drawback that such images usually have low spatial resolutions. In order to improve the spatial resolution, a Sub-pixels Analysis (SA) tool has been developed, taking into account the underlying digital topography, together with some derived attributes. The tool was applied to monitoring a flood event in the Senegal River Valley. A significant improvement in the flooded areas delineation with SA was achieved, compared with a supervised classification method that used the same satellite images. 


\section{MOTIVACIÓN Y OBJETIVOS}

El continente Africano se caracteriza por su compleja topografía y por los marcados gradientes de vegetación, coberturas del suelo, precipitación, etc. Esta diversidad afecta significativamente la dinámica atmosférica de escala sinóptica y el forzamiento climático del océano (Sylla et al., 2010b). El continente es conocido por sus extensas zonas semiáridas, donde la variabilidad de la precipitación amenaza los recursos hídricos.

Entre otras regiones, el Sahel (África Occidental) fue una de las más duramente golpeadas por la variabilidad climática durante el siglo XX. Ello se ha debido principalmente a la estrecha relación entre el clima y las actividades socioeconómicas, siendo la población en África Occidental más vulnerable a la variabilidad de la precipitación y sus cambios debidos al calentamiento global. La región ha sufrido severas sequías desde finales de la década de los ' 60 , si cabe más graves porque el rápido crecimiento de la población incrementa las presiones sobre el medio ambiente y los recursos naturales, acarreando profundos cambios en los usos del suelo. Durante el periodo 1990-2020, se espera que la población de la zona se duplique, con pronósticos de crecimiento aún mayores para algunas ciudades en el Valle del Río Senegal (Cour, 2001). Las fluctuaciones climáticas en el Sahel han tenido un efecto devastador (hambrunas, refugiados climáticos y lento crecimiento económico), revelando la gran vulnerabilidad de la región (Lebel et al., 2009; Paeth et al., 2009).

Debido a que la evidencia sobre la intensificación del ciclo hidrológico es robusta, es necesario mejorar la destreza para el seguimiento y la predicción de los impactos asociados con el cambio del régimen hidrológico (Huntington, 2006). El incremento de las tasas de precipitación y evaporación, causado por el calentamiento global, se está convirtiendo en una amenaza para la población, dado el incremento en la frecuencia de los eventos extremos (Kundzewicz et al., 2007). Estas amenazas están afectando de una manera desigual a los países en vías de desarrollo, dónde los recursos para adoptar medidas de adaptación o mitigación son limitados (Huntington, 2006).

Por todo lo anterior, el estudio del aumento en los gases de efecto invernadero, y la respuesta del clima, ha recibido mayor atención en la región en los últimos años. Además, en estas condiciones, la presunción de estacionariedad climática no puede ser garantizada debido a la interacción de diferentes forzamientos (entre otros, cambios en el uso del suelo y el crecimiento de la población). En este sentido, los RCMs se han convertido en una importante herramienta para mejorar el entendimiento de los procesos clave involucrados en la descripción de los mecanismos climáticos 
(Sánchez et al., 2009), para la simulación de escenarios plausibles con una resolución apropiada para el estudio de impactos a escala de cuenca. Sin embargo, los RCMs construidos para el África Occidental aún son sensibles a la parametrización física, la resolución espacio-temporal y la variabilidad interna (Paeth et al., 2011).

Es necesario, entonces, avanzar en el conocimiento de los impactos climáticos que afectarán la región. Disponer, para las comunidades afectadas en el África Occidental, del conocimiento sobre los riesgos climáticos, permitirá el desarrollo de estrategias encaminadas a construir "capacidad adaptativa" para la gestión. De acuerdo con el Panel Intergubernamental del Cambio Climático (Intergovernmental Panel on Climate Change, IPCC), la "capacidad adaptativa" puede ser entendida como la "habilidad para tolerar, adaptarse o recuperarse de los efectos de un riesgo o peligro", o en este caso, del cambio climático. La capacidad adaptativa es considerada una condición necesaria para diseñar e implementar estrategias efectivas de adaptación, y puede ser lograda incrementando el conocimiento sobre los riesgos climáticos potenciales que se ciernen sobre las diferentes cuencas (European Commission, 2009).

Considerando la motivación descrita, el objetivo principal del Proyecto de Tesis Doctoral consiste en mejorar las predicciones de los impactos del cambio y variabilidad climática en los eventos meteorológicos extremos. El estudio se centra en la Cuenca del Río Senegal (África Occidental).

Como objetivos complementarios, se han planteado los siguientes:

- Integrar la información de precipitación diaria, a partir de campos o mallas derivados desde Modelos Climáticos Regionales y Globales, en un ambiente de Sistemas de Información Geográfica (SIG).

- Obtener los patrones espacio-temporales de la precipitación en la zona de estudio, analizando el comportamiento interanual y las tendencias de las propiedades estadísticas de la lluvia.

- Caracterizar la tipología de eventos pluviométricos, y analizar los cambios en su frecuencia temporal.

- Desarrollar una metodología de aplicación de los resultados obtenidos para la toma de decisiones, gestión del riesgo y estrategias de adaptación. Ello conllevará el desarrollo de herramientas informáticas en base a imágenes de satélite y SIG, en conjunto con Modelos de Elevación Digital (Digital Elevation Model, DEM), para la detección de zonas inundadas.

Cada uno de los artículos del compendio tiene objetivos específicos que se han definido en su correspondiente resumen. 


\section{ANTECEDENTES Y ESTADO DEL ARTE}

\subsection{El Clima de África Occidental}

La distribución espacial de la precipitación se relaciona con la ocurrencia de Sistemas Convectivos de Mesoescala (Mesoscale Convective Systems, MCS) y con el forzamiento de la topografía. La variabilidad temporal está modulada por el ciclo estacional impuesto por el desplazamiento meridional de la Zona de Convergencia Intertropical (Intertropical Convergence Zone, ITCZ). En el invierno boreal (DJF), la ITCZ alcanza su posición más austral, entonces las áreas al norte del ecuador son predominantemente secas, entre ellas África Occidental. La ITCZ luego migra hacia el Norte, alcanzando su posición más septentrional en el verano boreal (JJA), cuando el Monzón de África Occidental (West African Monsoon, WAM) presenta máxima actividad.

Entender la complejidad del WAM es de crucial importancia para la estabilidad de una región, cuya economía es principalmente agrícola. La dinámica del WAM, y su conexión con la variabilidad de la precipitación, ha sido explicada por varios autores (Diedhiou et al., 1999; Paeth et al., 2005; Lebel and Ali, 2009). La ITCZ es el principal forzamiento que controla la precipitación de gran escala sobre África Occidental. Sin embargo, a escala regional, la variabilidad interanual de la precipitación está determinada también por el Chorro del Este de África (African Easterly Jet, AEJ; Lebel and Ali, 2009; Grist and Nicholson, 2001). El AEJ aparece durante el verano boreal como resultado del fuerte gradiente meridional de humedad superficial y temperatura entre el Desierto del Sahara y el Golfo de Guinea (Sylla et al., 2010b). Para la zona del Sahel, la formación de esta corriente es de suma importancia porque las bandas tormentosas de los MCSs se alinean al norte del AEJ. Durante los años más secos en la región, se ha observado que el $A E J$ se encuentra más cerca del ecuador que en los años húmedos (Sylla et al., 2010a).

De acuerdo con Paeth et al., (2005), diferentes factores interactúan no sólo en la generación del AEJ, sino también en el origen de las Ondas Tropicales del Este (African Easterly Waves, AEW) y el WAM. Estos factores incluyen la Temperatura Superficial del Océano (Sea Surface Temperature, SST), cambios en la cobertura del suelo, fenómenos tropicales de gran escala como El Niño - Oscilación del Sur (El Niño - Southern Oscillation, ENSO), y extratropicales como la Oscilación del Atlántico Norte (North Atlantic Oscillation, NAO). En cualquier caso, los diferentes fenómenos climáticos (WAM, AEJ, AEW e ITCZ) deberían ser observados como un único sistema con múltiples interconexiones. 
La presente Tesis ha concentrado su estudio en la Cuenca del Río Senegal, ubicada en el extremo occidental de África. El régimen hidrológico del Río Senegal es muy irregular, forzado por la variabilidad climática impuesta por el WAM. La precipitación media anual al interior de la cuenca presenta un fuerte gradiente latitudinal, creciente hacia el Sur, debido a las condiciones extremadamente secas impuestas por el Desierto del Sahara al Norte, y la humedad de la zona montañosa y las aguas del Golfo de Guinea al Sur (Figura 1a). Vinculado con este gradiente de la precipitación, se han identificado tres ecorregiones al interior de la cuenca (Figura 1b), de acuerdo con el trabajo de Olson et al. (2001): Sabana Boscosa Guineana (Guinean Forest Savanna, GFS, precipitación media anual $P>1000 \mathrm{~mm} / \mathrm{año}$ ); Sabana Sudaniana Occidental (West Sudanian Savanna, WSS, $P$ entre 600-1000 mm/año); y Sabana de Acacias del Sahel (Sahelian Acacia Savanna, SAS, $P$ entre 100-600 mm/año). La principal fuente de recursos de la cuenca se encuentra en las montañas del Fouta Djalon, al interior de la GFS, donde se pueden superar los 2000 mm/año, mientras que en el Sahel se tienen zonas con precipitación por debajo de 200 mm/año (Andersen et al., 2001). El Valle del Río Senegal está totalmente contenido en la zona árida del Sahel. En el valle se asienta una población continuamente creciente, que depende de la agricultura de recesión. El valle es una zona donde la degradación del suelo y la pérdida de cobertura vegetal es un problema persistente, debido a la aridez del terreno, las prácticas agrícolas inadecuadas y el sobrepastoreo (Lebel et al., 2009). En el futuro, estos problemas ambientales se agravarán, porque los ciclos de sequía e inundaciones serán cada vez más frecuentes e intensos debido al calentamiento global.

Debido a la vulnerabilidad de la región, el entendimiento de la variabilidad climática contribuirá para reducir las amenazas sobre la seguridad alimentaria de la población. Aunque los impactos sobre la región de la variabilidad climática observada y el calentamiento global están bien documentados (Descroix et al., 2009; Favreau et al., 2009; Séguis et al., 2011), y existe evidencia de respuestas desiguales de los sistemas hidrológicos, especialmente en la región del Sahel del África Occidental (Leduc et al., 2001; Mahé et al., 2005; Mahé and Paturel, 2009), aún debe profundizarse en el estudio de la variabilidad debida al cambio climático futuro, y sus impactos sobre la escorrentía. Este asunto es una de las principales cuestiones abordadas por el Proyecto Europeo de Investigación Análisis Multidisciplinario del Monzón Africano (African Monsoon Multidisciplinary Analysis, AMMA; Polcher et al., 2011). El Proyecto AMMA, en estrecha colaboración con el Proyecto Europeo ENSEMBLES, permitió la construcción de modelos climáticos de alta resolución que simularan adecuadamente 
el gradiente climático que se observa en África Occidental, con el objetivo de abordar adecuadamente los retos impuestos por el calentamiento global.
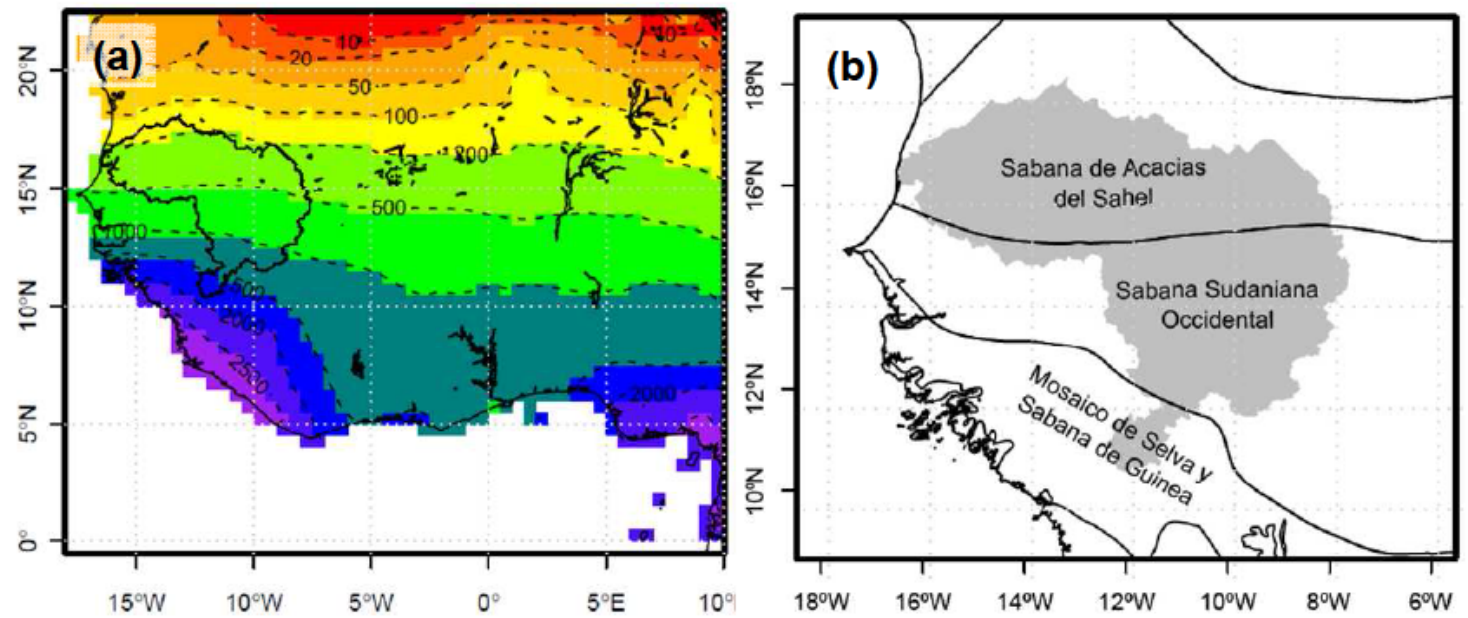

Figura 1. Zona de estudio: (a) Precipitación media anual $(\mathrm{mm})$ en África Occidental en el periodo 1961-1990 (base de datos CRU). (b) Ecorregiones al interior de la Cuenca del Río Senegal.

\subsection{Modelos Climáticos}

\subsubsection{Modelos de circulación general}

Un Modelo de Circulación General (General Circulation Model, GCM) es una abstracción matemática compleja de los componentes del sistema climático a nivel planetario (atmósfera, océanos, criosfera, geosfera y biosfera.). Los GCM resuelven ecuaciones diferenciales basadas en leyes básicas de la física, la dinámica de fluidos y la química sobre una malla (o grilla) tridimensional (ver Figura 2) Cada una de las celdas de la malla representa una ubicación geográfica y una altura determinadas. En cada una de ellas, se resuelven ecuaciones específicas para cada componente climático y se calcula la evolución de diferentes variables climáticas con el tiempo, permitiendo el intercambio de flujos de calor, masa y momento entre las celdas, interactuando como un sistema acoplado.

La principal ventaja de los GCM es su capacidad para incorporar información histórica para determinar el estado real de la atmósfera terrestre (como lo haría la asimilación de datos para ejecutar un reanálisis meteorológico), o ejecutar múltiples experimentos utilizando como forzamiento diferentes escenarios de emisión de gases de efecto invernadero (greenhouse gases emission, GHGE). El rango y la probabilidad asociada con las tendencias climáticas es mejor entendido si se ejecutan múltiples experimentos, con diferentes GCM, forzados con distintos escenarios GHGE. 


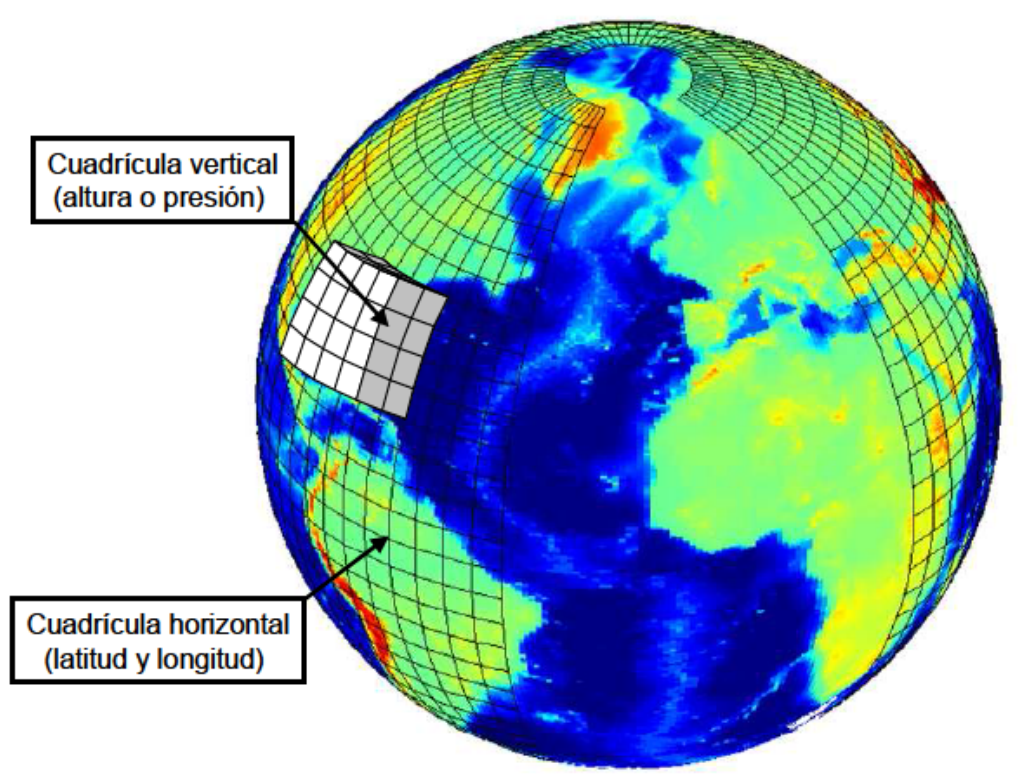

Figura 2. Representación esquemática de un GCM. El planeta es dividido en una malla esférica tridimensional. En cada celda de la malla se ejecutan diferentes proceso físicos del modelo.

EI IPCC fue creado en 1988 por la Organización Meteorológica Mundial (World Meteorological Organization, WMO) y el Programa Ambiental de las Naciones Unidas (United Nations Environment Programme, UNEP) con el objetivo de evaluar científicamente los efectos y los aspectos socio-económicos del cambio climático, así como estudiar y proponer alternativas de mitigación y adaptación.

El Informe Especial sobre Escenarios de Emisión (Special Report on Emissions Scenarios, SRES) del IPCC (IPCC SRES, 2000), definió los GHGE. Estos son establecidos por forzamientos tales como el desarrollo demográfico, socioeconómico y tecnológico. A pesar de la profunda reflexión para elaborarlos, su evolución futura es altamente incierta, y ninguno de ellos es considerado, por ahora, más probable de ocurrir. El documento agrupa los GHGE en "familias de escenarios", que se pueden resumir de acuerdo con la Tabla 1.

La familia A1 supone integración global. Está caracterizada por un rápido crecimiento económico, un pico de población en el año 2050 (9 mil millones de personas) para luego declinar gradualmente, una rápida asimilación global de tecnologías novedosas y eficientes, y un mundo convergente (en ingresos económicos y estilo de vida debido a la globalización cultural). Dentro de la familia A1 existen subconjuntos de escenarios, de acuerdo con sus énfasis tecnológicos: A1FI (combustibles fósiles), A1B (balance entre distintas fuentes de energía) y A1T (combustibles no fósiles).

La familia A2 de escenarios supone un mundo muy heterogéneo. Se caracteriza por un mundo con naciones autosuficientes que actúan de forma independiente, con 
menor intercambio cultural, incremento constante de la población y desarrollos económicos y tecnológicos enfocados regionalmente (los más lentos entre todas las familias de escenarios).

La familia B1 de escenarios supone un mundo ampliamente integrado, y mucho más amigable con el medio ambiente. Se caracteriza por un rápido crecimiento económico, tanto como la familia $A 1$, pero con un rápido giro hacia una economía de servicios e información. El comportamiento de la población es igual que la familia A1, se reduce la consumición de materiales y se introducen tecnologías limpias y eficientes para explotar los recursos. Finalmente, triunfan las soluciones globales para garantizar la estabilidad económica, social y ambiental.

La familia B2 de escenarios supone un mundo fragmentado, pero amigable con el ambiente. Se caracteriza por un incremento constante de la población, aunque menor que en la familia $\mathrm{A} 2$, se imponen las soluciones locales para garantizar la sostenibilidad económica, social y ambiental. El nivel de desarrollo económico es intermedio, y el intercambio tecnológico es más lento y fragmentado que en las familias $A 1$ y $B 1$.

Tabla 1. Familias de escenarios de GHGE del SRES. Se presenta el rango proyectado de aumento de las temperaturas para el año 2100.

\begin{tabular}{|c|c|c|}
\hline & $\overline{\text { Enfoque más económico }}$ & Enfoque más ambiental \\
\hline \multirow{3}{*}{$\begin{array}{c}\text { Globalización } \\
\text { (homogeneidad } \\
\text { global) }\end{array}$} & $\overline{A 1}$ & B1 \\
\hline & $\begin{array}{l}\text { Rápido crecimiento económico } \\
\text { (arupos } \mathrm{A} 1 \mathrm{~T}, \mathrm{~A} 1 \mathrm{~B}, \mathrm{~A} 1 \mathrm{FI})\end{array}$ & Sostenibilidad ambiental global \\
\hline & {$[1.4 ; 6.4]^{\circ} \mathrm{C}$} & {$[1.1 ; 2.9]^{\circ} \mathrm{C}$} \\
\hline \multirow{3}{*}{$\begin{array}{c}\text { Regionalización } \\
\text { (heterogeneidad } \\
\text { global) }\end{array}$} & A2 & B2 \\
\hline & Desarrollo económico regional & Sostenibilidad ambiental a nivel \\
\hline & {$[2.0 ; 5.4]^{\circ} \mathrm{C}$} & {$[1.4 ; 3.8]^{\circ} \mathrm{C}$} \\
\hline
\end{tabular}

Líneas evolutivas estimadas para cada escenario son presentadas en el Informe Resumido del $4^{\circ}$ Informe de Valoración del IPCC (IPCC AR4, 2007), donde se representa la evolución promedio del calentamiento superficial (relativo al periodo 1980-1999), presentados como una continuación de la simulación para el siglo XX, así como el rango definido por una desviación estándar alrededor del promedio, obtenidos desde las simulaciones.

Está bien aceptado por la comunidad científica que los GCM tienen capacidades para simular la distribución sinóptica de las principales variables climáticas y aspectos del cambio climático observado. Sin embargo, presentan deficiencias a escalas regionales 
debido a su gruesa resolución espacial (generalmente del orden de cientos de kilómetros). A pesar de que la capacidad computacional se ha incrementado, los GCM no simulan adecuadamente características climáticas de mesoescala. Además, algunas veces estos modelos simplifican o parametrizan inadecuadamente procesos climáticos no lineales complejos. Estas desventajas, que se atribuyen principalmente a la baja resolución espacial, se han intentado superar mediante la construcción de RCM con resoluciones más finas, anidados en los GCMs. De esta manera, los GCMs imponen las condiciones de frontera (o contorno) a los RCMs.

\subsubsection{Modelos climáticos regionales para África Occidental}

El downscaling de un GCM usando un RCM es una práctica común para obtener información de alta resolución acerca de los escenarios de cambio climático proyectado. De acuerdo con Rummukainen (2010), las técnicas de downscaling pueden agruparse en estadísticas y dinámicas. Las primeras requieren de relaciones estadísticas fuertes entre las variables del clima de gran escala (e. g. el nivel medio del mar, los campos de presión, etc.) y las locales (temperatura, precipitación, etc.). El downscaling dinámico, de acuerdo con Herrera et al. (2010), consiste en solucionar las ecuaciones de la física climática con una resolución espacial mejorada para una región particular, utilizando algún GCM como condición de contorno.

Para África Occidental, los RCM fueron construidos por el experimento RT3 del Proyecto Europeo ENSEMBLE (Christensen et al., 2009) en estrecha colaboración con el Proyecto AMMA (Polcher et al., 2011). El Proyecto ENSEMBLE se benefició al contar con información para evaluar sus modelos, diseñados originalmente para Europa, en otro régimen climático. Para la mayoría de las simulaciones, el dominio fue $19.8^{\circ} \mathrm{S}-35.2^{\circ} \mathrm{N}$ y $35.2^{\circ} \mathrm{W}-312^{\circ} \mathrm{E}$ (Figura 3). África Occidental tiene un gradiente climático muy fuerte, como muestra la Figura 1(a), desde el Golfo de Guinea al sur, hasta el Desierto del Sahara al norte, el cual no es resuelto adecuadamente por los GCMs. Por lo tanto era interesante evaluar si los RCMs con resolución espacial más fina podían reducir las incertidumbres y aumentar la confianza en las predicciones climáticas futuras. Los RCMs fueron forzados con datos ERA-Interim (Dee et al., 2011) con propósitos de evaluación, lo que significó que sólo el periodo 1990-2007 estaba disponible para este tipo de simulaciones. La base de datos ERA-Interim fue utilizada porque ha demostrado mejorar significativamente la simulación del ciclo hidrológico en zonas tropicales (Christensen et al., 2009). A futuro se ejecutaron simulaciones con 50 km de resolución espacial entre 1991-2050, las cuales fueron forzadas de acuerdo con SRES-A1B y tres GCMs. 


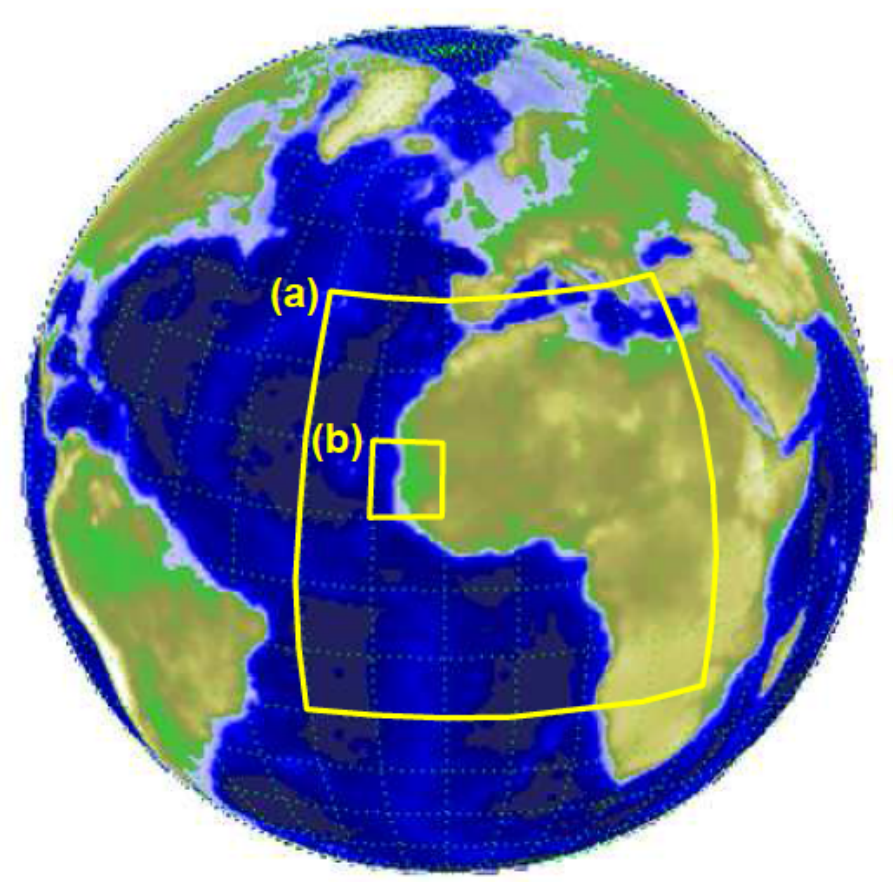

Figura 3. África Occidental. (a) Dominio espacial de los RCM construidos para la región dentro del experimento RT3 del Proyecto ENSEMBLES. (b) Ubicación de la Cuenca del Río Senegal.

Las características de los diferentes RCMs utilizados en esta Tesis, junto con las bases de datos observados, se resumen en la Tabla 2. La selección de los RCMs estuvo basada en su coincidencia temporal con la base de datos observados IRD (Institut de Recherche pour le Développement, Francia). Todas las bases de datos están constituidas por mallas de datos espacialmente continuas para África Occidental, excepto los datos IRD. Los datos IRD presentaron discontinuidad espacial que restringió los análisis de sesgo realizados con ellos en varios trabajos que constituyen la Tesis (García Galiano and Giraldo Osorio, 2010; Karambiri et al., 2011; García Galiano and Giraldo Osorio, 2011; Giraldo Osorio and García Galiano, 2011).

Una vez publicados los datos RCM, comenzaron los estudios sobre impactos del cambio climático en África Occidental. La Universidad Politécnica de Cartagena (UPCT, España) se sumó a esta iniciativa como socio del Proyecto AMMA, como subraya el documento de Christensen et al. (2009):

"2iE in Ouagadougou and UPCT in Cartagena concentrate on the regional climate change simulations. They look at the projected evolution of the dry spells which occur during the rainy season and how the intra-seasonal characteristics of the monsoon might evolve in a warmer climate. Also water resources impact studies are made for the upper Volta basin (The Nakambé) for estimates of water resources until 2050" 
“El 2iE (Institut International de l'Ingénierie de l'Eau et de l'Environnement) en Ouagadougou (Burkina Faso) y la UPCT en Cartagena (España) se concentran en la simulación del cambio climático a nivel regional. Ellos estudian la evolución proyectada de las rachas secas que ocurren durante la temporada lluviosa y como las características intra-estacionales del monzón evolucionarán enmarcadas por un clima más cálido. Además, llevarán a cabo estudios del impacto sobre los recursos hídricos para la cuenca alta del Río Volta (Río Nakambé) para estimar la disponibilidad de los recursos hasta el año 2050".

Tabla 2. Resumen de los RCMs utilizados, junto con las características de las bases de datos observados IRD y CRU.

\begin{tabular}{c|c|c|c|c|c}
\hline \hline Nombre & Instituto & GCM & RCM & $\begin{array}{c}\text { Resolución } \\
\text { espacial }\end{array}$ & $\begin{array}{c}\text { Cobertura } \\
\text { temporal }\end{array}$ \\
\hline \hline IRD & $\mathrm{IRD}^{(1)}$ & \multicolumn{2}{|c|}{ Datos observados diarios } & $1^{\circ}(\sim 100 \mathrm{~km})$ & $1970-1990$ \\
$\mathrm{CRU}$ & $\mathrm{CRU}^{(2)}$ & \multicolumn{2}{|c|}{ Datos observados mensuales } & $0.5^{\circ}(\sim 50 \mathrm{~km})$ & $1960-2000$ \\
$\mathrm{GKSS} / \mathrm{CLM}$ & $\mathrm{GKSS}^{(3)}$ & ECHAM5 & $\mathrm{CLM}$ & $50 \mathrm{~km}$ & $1961-2050$ \\
$\mathrm{INM} / \mathrm{RCA}$ & $\mathrm{INM}^{(4)}$ & HadCM3Q0 & $\mathrm{RCA}$ & $50 \mathrm{~km}$ & $1951-2099$ \\
$\mathrm{METO} \mathrm{HC} / \mathrm{HAD}$ & $\mathrm{HC}^{(5)}$ & HadCM3Q0 & HadRM3P & $50 \mathrm{~km}$ & $1951-2099$ \\
$\mathrm{KNMI/RACMO}$ & $\mathrm{KNMI}^{(6)}$ & ECHAM5-r3 & RACMO & $50 \mathrm{~km}$ & $1970-2050$ \\
$\mathrm{MPI}-\mathrm{M} / \mathrm{REMO}$ & $\mathrm{MPI}^{(7)}$ & ECHAM5-r3 & REMO & $50 \mathrm{~km}$ & $1950-2050$ \\
SMHI/RCA & $\mathrm{SMHI}^{(8)}$ & HadCM3Q0 & RCA & $50 \mathrm{~km}$ & $1951-2100$ \\
\hline \hline
\end{tabular}

${ }^{(1)}$ Institut de Recherche pour le Développement, Francia. ${ }^{(2)}$ Climate Research Unit, University of East Anglia, Reino Unido. ${ }^{(3)}$ GKSS Research Centre, Alemania. ${ }^{(4)}$ Instituto Nacional de Meteorología, España. ${ }^{(5)}$ Hadley Centre, Reino Unido. ${ }^{(6)}$ Koninklijk Nederlands Meteorologisch Instituut, Países Bajos. ${ }^{(7)}$ Max-Planck-Institut für Meteorologie, Alemania. ${ }^{(8)}$ Swedish Meteorological and Hydrological Institute, Suecia.

Aunque se han logrado notables avances en la simulación de la dinámica del WAM (Fontaine et al., 2011; Paeth et al., 2011; Ruti et al., 2011), aún se observan dispersiones importantes entre los resultados de los RCMs, lo que incrementa la incertidumbre de las predicciones. Además, los RCMs exhiben niveles de "habilidad" desiguales en la simulación de los procesos, sobre diferentes regiones y considerando variables hidrometeorológicas distintas, haciendo difícil identificar los modelos más confiables. En consecuencia, se justifica la construcción de ensembles que consideren la variabilidad simulada por todos los modelos climáticos. Las proyecciones climáticas y la estimación de las incertidumbres asociadas están mejor basadas en la combinación de la información suministrada utilizando una aproximación ensemble desde las diferentes simulaciones de RCMs (Giorgi and Mearns, 2002; Paeth et al., 2011). Finalmente, las tendencias observadas en las variables hidrometeorológicas obligan a cuestionar los análisis estadísticos estacionarios. Deben utilizarse 
herramientas innovadoras que permitan simular la no estacionariedad de los parámetros de las distribuciones.

\subsection{Análisis No Estacionario}

El calentamiento global ha transformado los patrones de la precipitación y, en consecuencia, ha cambiado la frecuencia y magnitud de los eventos extremos (Labat et al., 2004; Huntington, 2006; Kundzewicz et al., 2007). Coincidiendo con la opinión de varios autores (Milly et al., 2008; Villarini et al., 2010), deberían utilizarse modelos probabilísticos no estacionarios capaces de reproducir la variación temporal de los parámetros de las distribuciones de probabilidad asociadas con estas variables.

La estacionariedad de las series hidrológicas no puede ser garantizada, por lo que se requiere una metodología de modelización que permita considerar la no estacionariedad de los parámetros de las distribuciones de probabilidad. En esta Tesis, se han utilizado GAMLSS, suponiendo distribuciones de probabilidad paramétricas para la variable explicada (en nuestro caso, $Y=A M D S L$ ó $Y=A M D R$ ), cuyos parámetros se han modelado como función de la variable explicativa (tiempo $t$ ) utilizando splines cúbicos (cubic-spline) como funciones de suavizado. Una ampliación de los aspectos teóricos de GAMLSS es presentada por Rigby and Stasinopoulos (2005) y Stasinopoulos and Rigby (2007), mediante una detallada discusión sobre la selección y ajuste del modelo estadístico.

El número de parámetros utilizados para ajustar los modelos estadísticos depende del tipo de distribución escogida, pero suele ser menor que cuatro (el primer parámetro de ubicación, el segundo de escala, y el tercero y cuarto de forma). Se ha considerado que no se justifican distribuciones con más de dos parámetros debido a la corta longitud de los registros (81 observaciones anuales entre 1970-2050), dificultando el correcto ajuste de los parámetros de forma. Por lo tanto, se seleccionaron cuatro distribuciones de dos parámetros ampliamente utilizadas para la modelización estadística de series hidrológicas (Gamma -GA-, Gumbel -GU-, Lognormal -LN-, Weibull -WEI-), que se esperaba tuvieran una flexibilidad suficiente para describir los datos de las variables escogidas. La relación entre los parámetros de la distribución con $E[Y]$ and $\operatorname{Var}[Y]$, es explicada por Stasinopoulos et al. (2008).

No se supuso una relación lineal de los parámetros con el tiempo. En lugar de eso, se utilizó la formulación de GAMLSS aditiva semiparamétrica en la cual los parámetros son función del tiempo a través de cubic-spline optimizados. El uso de los cubic-spline debe hacerse con cautela, ya que otorgar valores altos a los grados de libertad efectivos $\lambda$ puede suponer una pérdida importante de la parsimonia del modelo. Debe 
anotarse que cuando $\lambda$ tiende a cero, los cubic-spline tienden a una línea recta, por lo que se puede considerar que la dependencia lineal de los parámetros con el tiempo está incluida y es un caso especial de los cubic-spline. Siguiendo el procedimiento sugerido por Stasinopoulos and Rigby (2007), se ajustaron los modelos utilizando el Criterio Schwarz Bayesian (Schwarz Bayesian Criterion, SBC), limitando los grados de libertad efectivos a $\lambda \leq 4$.

Una vez obtenido $\lambda$ para cada una de las distribuciones consideradas en cada punto, se ha seleccionado aquella que tuviera el mayor valor de máxima verosimilitud. Sin embargo, la máxima verosimilitud no es un indicador definitivo sobre la calidad del ajuste, por lo que debe analizarse la independencia y normalidad de los residuos de cuantiles aleatorios normalizados (Rigby and Stasinopoulos, 2005), para asegurar que el modelo seleccionado describe adecuadamente los datos. En esta Tesis, se ha verificado la independencia y normalidad de los residuos calculando su media, varianza, asimetría, curtosis, y el coeficiente de correlación de Filliben (Filliben, 1975). También la inspección de los qq-plot (gráficos de cuantiles) y los worm-plot (van Buuren and Fredriks, 2001) se llevó a cabo para verificar la normalidad de los residuales. El paquete GAMLSS para $R$, fue utilizado para realizar el modelado estadístico no estacionario descrito (Stasinopoulos et al., 2008).

La Figura 4 presenta un ejemplo del análisis GAMLSS, junto con las pruebas de normalidad de los residuos. Este gráfico pertenece al análisis realizado en el artículo Giraldo Osorio and García Galiano (2011), considerado como material suplementario (http://www.hydrol-earth-syst-sci.net/15/3605/2011/hess-15-3605-2011.html).

En la Figura 4 se presentan un ejemplo de análisis de residuales (estadísticos básicos, qq-plot y worm-plot). El coeficiente de correlación de Filliben es una medida simple y rápida de la independencia de los residuales (Filliben, 1975). Se calcula asignando valores de la fórmula de Beard (Makkonen, 2006) para posiciones de graficación a la serie ordenada de los residuales, y luego calculando el coeficiente de correlación entre estos valores y la serie ordenada. Para un nivel de significancia del $5 \%$ y un tamaño muestral $n=80$, se rechaza la hipótesis de normalidad si el coeficiente es menor que 0.984. Los qq-plot verifican la normalidad al comparar gráficamente la distribución teórica (en este caso, normal), con la distribución empírica de los residuales. Si la distribución de los residuales efectivamente es normal, estos deberían ubicarse muy cerca de la recta de pendiente unitaria. De acuerdo con van Buuren and Fredriks (2001), los worm-plot son qq-plot sin tendencia. La forma que adopte el worm-plot indica cómo difieren los datos de la distribución supuesta (normal en este caso). Una 
cadena de puntos plana, con todos los residuales entre las bandas de confianza (Figura 4b) indica un adecuado ajuste de los datos a la distribución supuesta.
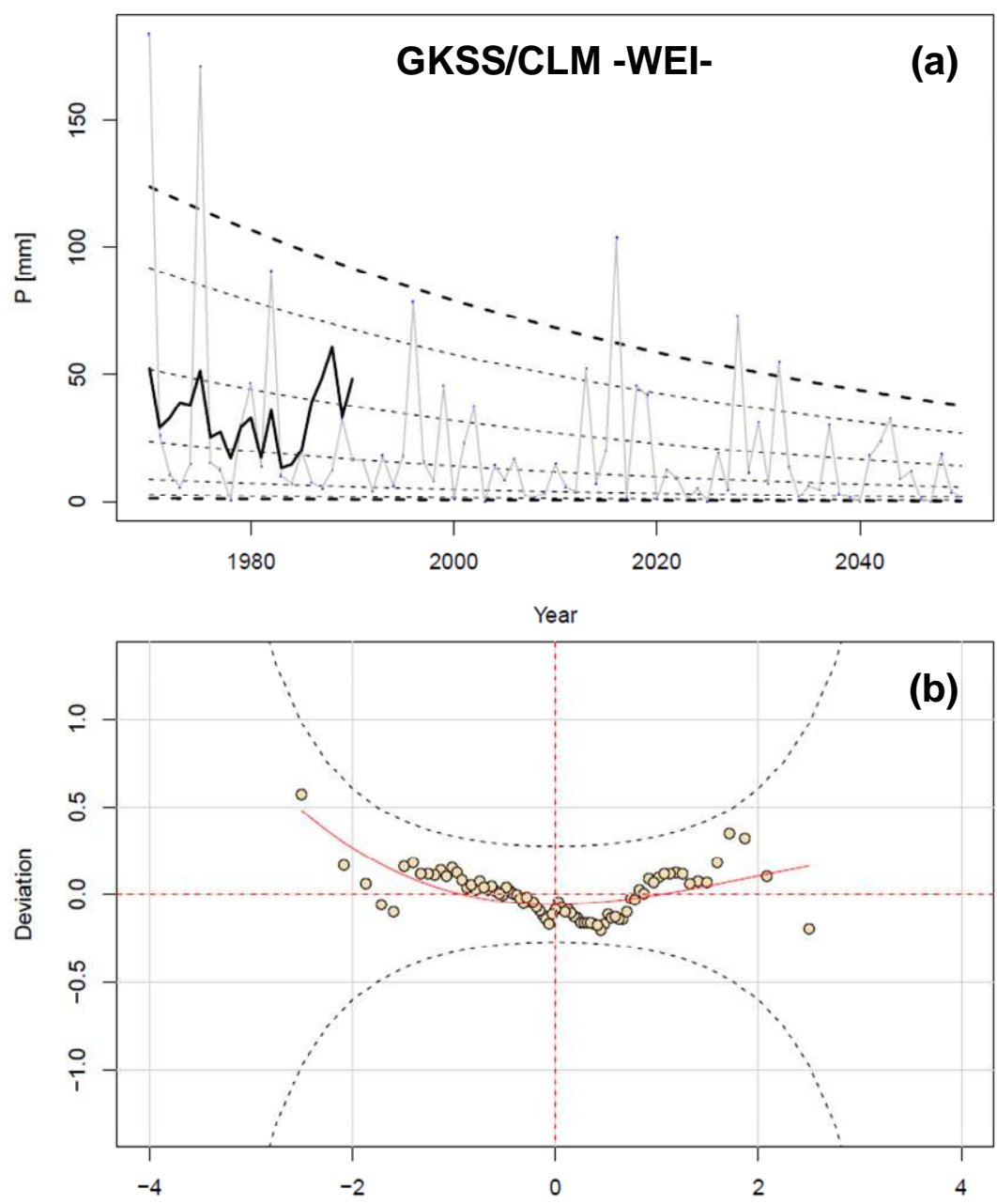

Unit normal quantile

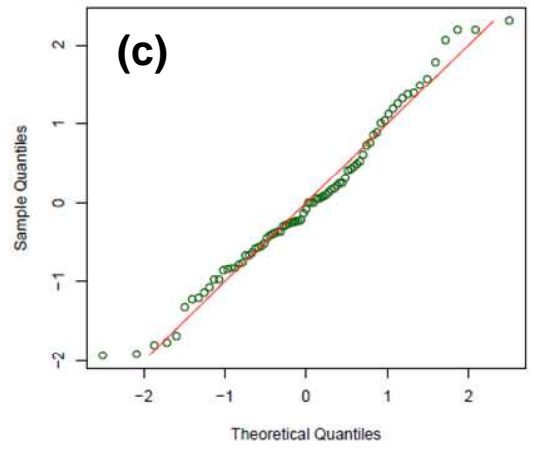

Summary of the Quantile Residuals mean $=0.003766$

var $=0.962047$

skewness $=0.3317$

kurtosis $=2.807349$

filliben correlation coefficient $=0.991344$

Figura 4. Ejemplo de análisis GAMLSS a la serie de AMDR del sitio 16 para el GKSS/CLM RCM. (a) Serie temporal de AMDR para el RCM (gris) y para los datos IRD (negro), en conjunto con las curvas de los centiles (5 al $95 \%$, en línea discontinua) que representan la distribución de probabilidad GAMLSS ajustada; (b) worm-plot y (c) qq-plot de los residuales. Se presentan además los estadísticos de los residuales. Se rechaza la hipótesis de normalidad si el coeficiente de Filliben es menor que 0.984 (Material suplementario en la discusión abierta del artículo Giraldo Osorio and García, 2011). 
Aunque la metodología GAMLSS permite simular la no estacionariedad de las PDF de las variables consideradas (AMDSL y AMDR), y obtener una medida de la incertidumbre del cambio para cada modelo, es necesario el uso de ensembles para simular toda la variabilidad, debido a la divergencia en los resultados obtenidos desde los diferentes RCM. El tratamiento matemático necesario para la construcción de PDF ensembles puede ser bastante difícil. Las técnicas de bootstrapping facilitan esta tarea al no requerir suposiciones sobre las distribuciones de los datos.

\subsection{Técnicas de Bootstrapping}

Las técnicas de bootstrapping (muestreos con reemplazo) son una aproximación de la inferencia estadística, basada en construir una distribución de la muestra con el objetivo de calcular un estadístico utilizando muestreos con reemplazo de los datos (Efron and Tibshirani, 1993; Fox, 2002). Si existe una función distribución de probabilidad ajustada a los datos, las muestras podrían ser números aleatorios obtenidos de estas PDF analíticas.

Fox (2002) presenta una definición matemática simple del bootstrapping no paramétrico (ver el Apéndice 3). Supóngase que $\mathbf{S}=\left\{X_{1}, X_{2}, \ldots, X_{n}\right\}$ es una muestra extraída de una población $\mathbf{P}=\left\{x_{1}, x_{2}, \ldots, x_{N}\right\}$. $\mathbf{P}$ puede ser una población infinita definida por una PDF. Ahora, se considerará que se está interesado en algún estadístico $T=t(\mathbf{S})$, el cual es un estimado del parámetro de la población $\theta=t(\mathbf{P})$. La estadística inferencial hará conjeturas sobre la estructura de la población (e. g. asumir normalidad), y a partir de ésta se derivará la distribución muestral de $T$. Esta aproximación puede tener dos importantes deficiencias. La primera, es evidente que si la hipótesis sobre la población es incorrecta, la distribución del estadístico obtenido de la muestra correspondiente será inexacta. Por otro lado, si la distribución asintótica es confiable, una muestra relativamente pequeña puede no llegar al nivel de precisión requerido. La segunda dificultad es la dificultad matemática asociada con esta aproximación. Se requiere una valiosa suficiencia matemática para derivar la distribución muestral del estadístico de interés. No obstante, en algunos casos será simplemente prohibitivo hacerlo. En contraste, el bootstrap no paramétrico permite estimar la distribución muestral del estadístico de forma empírica, sin hipótesis sobre la población y sin derivar la distribución muestral de forma explícita.

En principio, se podrían enumerar todas las muestras bootstrap de tamaño $n$. Sin embargo, el número de todas las posibles muestras bootstrap es excepcionalmente grande, a menos que $n$ sea muy pequeño. Si se tratan todos los elementos de una población como distintos (aún cuando existan varios elementos con el mismo valor), 
entonces existirán $n^{n}$ muestras bootstrap, cada una con una probabilidad de ocurrencia de $1 / n^{n}$. La inferencia bootstrap tiene, en consecuencia, dos fuentes de error. En primer lugar, el error producido por utilizar una muestra $\mathbf{S}$ particular para representar la población. La otra, es el error de muestreo resultado de la carente enumeración de todas las muestras bootstrap. Este último puede ser controlado replicando un número suficientemente grande de veces el muestreo bootstrap.

\subsection{Ensembles de Modelos Climáticos}

A pesar de los progresos logrados en la modelación climática con los RCMs, aún persisten sensibilidades a algunos parámetros físicos, a la resolución espacio-temporal y a la variabilidad interna de los modelos. Esto indica que una aproximación ensemble es necesaria para obtener información robusta sobre el cambio climático, y para estimar adecuadamente las incertidumbres (Paeth et al., 2011).

Un ensemble de modelos permite una descripción más precisa de las incertidumbres, así como una aproximación probabilística a los predicciones climáticas futuras (Sánchez et al., 2009). Tebaldi and Knutti (2007) realizaron una revisión de metodologías para la construcción de ensemble de modelos, comparando los resultados para proyecciones de temperatura. Sin embargo, algunas veces los errores calculados para cada modelo climático se compensan al combinarlos en un ensemble. Una ponderación de los modelos, basada en datos observados, puede mejorar este problema (Sánchez et al., 2009). Para asignar los factores de ponderación (o pesos) a los diferentes RCMs de un ensemble, es necesario comparar diferentes estadísticos con los calculados desde los datos observados.

No obstante, el uso de estadísticos como la media y la desviación estándar no permite la comparación completa de la distribución de los datos, aún cuando la evidencia ha demostrado que los cambios observados en los eventos extremos (en las colas de la distribución de sequías y precipitaciones máximas) son diferentes en magnitud que los observados en los valores medios. Se espera que los cambios asociados con los valores extremos tengan un mayor impacto sobre los sistemas biofísicos (Perkins et al., 2007; Tapiador et al., 2009). Esta situación justifica cuestionarse la habilidad de los RCMs para simular la distribución de probabilidad observada de las variables hidrometeorológicas. Distintos autores (Wigley and Raper, 2001; Giorgi and Mearns, 2003; Tebaldi et al., 2005; Giorgi, 2008; Buser et al., 2009), han abordado la estimación de PDFs asociadas con variables hidrometeorológicas utilizando proyecciones climáticas. Asumir que un modelo es más idóneo para simular la PDF observada de una variable, implica que se tendrá una mayor confianza en las 
proyecciones futuras de este modelo (Perkins et al., 2007; Sánchez et al., 2009). Sin embargo, la habilidad para representar el clima actual no parece ser suficiente para avalar su confiabilidad para simular el clima futuro (Giorgi and Mearns, 2003). Se respalda, por tanto, la investigación en la identificación de metodologías para construir ensembles con modelos climáticos.

\subsubsection{Desempeño de los modelos}

Básicamente, la combinación de los miembros de un ensemble puede realizarse de dos maneras. La primera de ellas es olvidar que los modelos tienen diferente habilidad para simular el clima observado, y ponderarlos igualmente (Murphy et al., 2004). La otra forma de hacerlo es utilizar promedios ponderados, donde el factor aplicado a cada modelo depende de alguna medida de desempeño. La cuestión de fondo es definir la métrica para cuantificar el desempeño de los modelos, porque no existe una única manera para hacerlo. Varios trabajos han abordado el problema desde diferentes puntos de vista (Räisanen and Palmer, 2001; Giorgi and Mearns, 2002; Giorgi and Mearns, 2003; Dettinger, 2005; Tebaldi et al., 2005; Sánchez et al., 2009). Sin embargo, las conclusiones pueden ser contradictorias debido a la desigual dispersión de los resultados, como opinan Tebaldi and Knutti (2007).

En este trabajo, se han construido las PDF ensemble utilizando la metodología REA (Reliability Ensemble Average) propuesta por Giorgi and Mearns (2002). Los diferentes factores del método (el factor de desempeño $R_{B}$, y el factor de convergencia $R_{D}$ ), fueron calculados utilizando distribuciones de probabilidad empíricas. Luego, aplicando técnicas de bootstrapping, se construyeron las PDF ensemble, utilizando las distribuciones no estacionarias ajustadas con GAMLSS a las series de los RCMs.

Las PDF ensemble de las AMDR, fueron construidas siguiendo el diagrama de flujo de la Figura 5. Se presenta el caso específico de esta variable ya que fue el más complicado de abordar. Inicialmente, se realiza la lectura de las simulaciones provistas por los RCMs, y los datos observados. Luego, el resultado del análisis del desvío es el valor del criterio de desempeño $\left(R_{B}\right)$, que es calculado con las PDF empíricas en el periodo 1961-1990. Las PDF empíricas en el periodo 2021-2050 son utilizadas para calcular el valor del criterio de convergencia $\left(R_{D}\right)$. En base a los valores de ambos criterios se calcula el valor del criterio de confiabilidad REA $(R)$, y el valor del factor de confiabilidad normalizado $(\mathrm{Pm})$. Siguiendo el análisis no estacionario en el diagrama de flujo, se observa que las series temporales entre 1960-2050 se utilizaron para ajustar las PDF no estacionarias a cada RCM aplicando GAMLSS. Estas PDF no estacionarias fueron luego utilizadas para construir las PDF ensemble, considerando como factor de ponderación el valor de $\mathrm{Pm}$ calculado para cada modelo. 


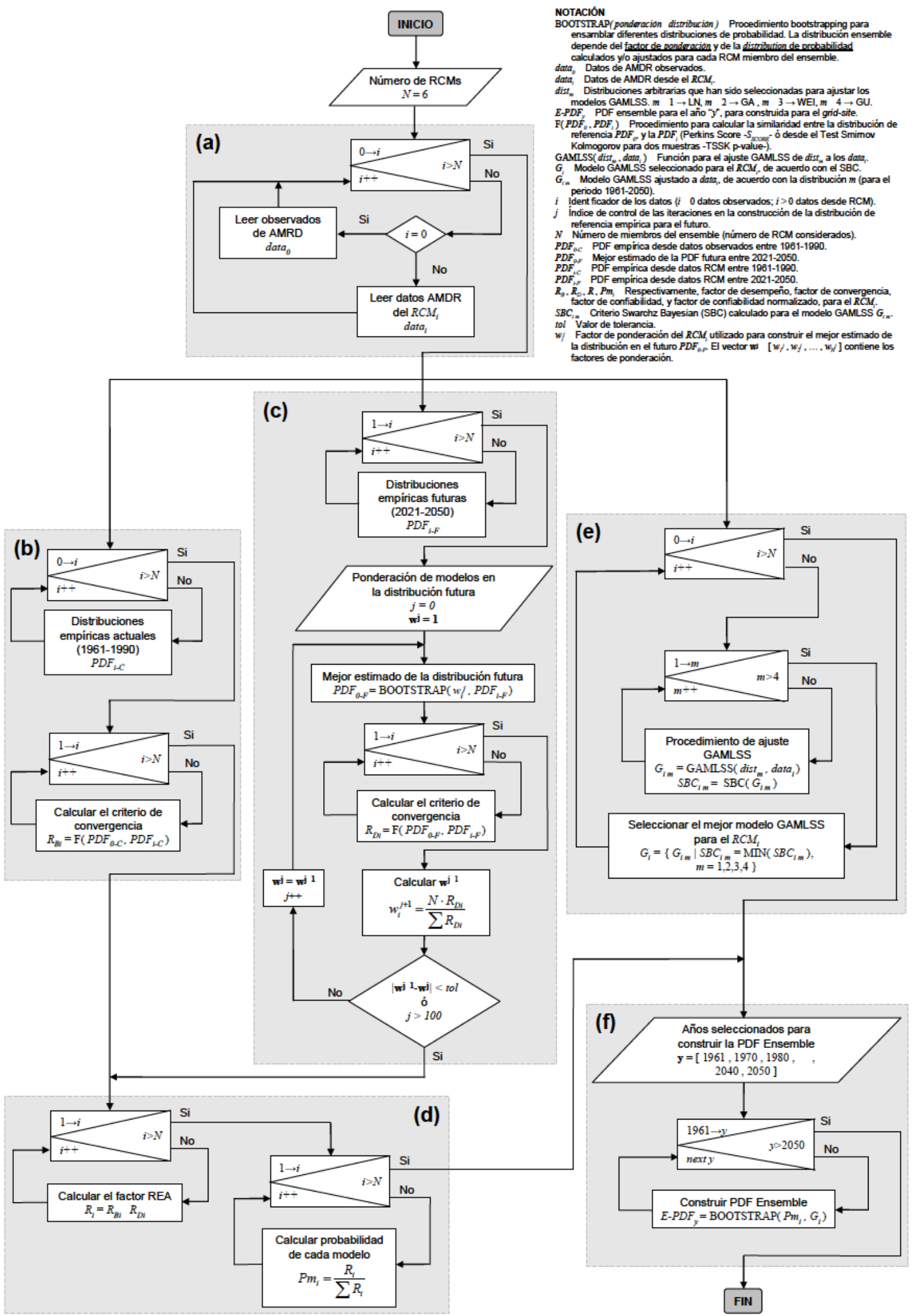

Figura 5. Diagrama de flujo para construir las PDF ensemble de las AMDR en cada sitio de análisis. Los diferentes procedimientos son: (a) lectura de los datos, (b) análisis del desvío, (c) análisis de convergencia a futuro, (d) cálculo REA, (e) análisis no estacionario, y (f) construcción de la PDF ensemble. 


\subsubsection{Factores de ponderación}

Como se ha comentado, se calcularon los factores de peso de los RCM para construir la ensemble PDF utilizando la metodología REA. El factor de confiabilidad del modelo $R$ para cada $\mathrm{RCM}$ se define como:

$$
R=\left[\left(R_{B}\right)^{b} \times\left(R_{D}\right)^{d}\right]^{1 /(b \times d)]}
$$

donde $R_{B}$ es una medida del desvío del modelo en base a datos observados (periodo 1961-1990); en otras palabras, a mayor desvío menor confiabilidad del modelo. $R_{D}$ es un factor que mide la confiabilidad de cada modelo en base a la distancia al "mejor estimado" de la respuesta del clima futuro, que es construido con las proyecciones climáticas de todos los RCMs considerados. La confiabilidad del modelo es menor a mayor distancia de este "mejor estimado". Dicho de otra forma, $R_{B}$ es una medida del desempeño, y $R_{D}$ es una medida de la convergencia a futuro (Giorgi and Mearns, 2002). Los parámetros $b$ y $d$ son la ponderación de cada criterio. Se asumió siempre que $b=d=1$, dando igual peso a ambos criterios.

La evaluación de los factores $R_{B}$ y $R_{D}$ se basó en funciones de distribución acumulada empíricas (cumulative distribution functions, CDF), y una medida cuantitativa que comparó el grado de ajuste entre las funciones de probabilidad. La fórmula de Weibull (Makkonen, 2006) para posiciones de graficación fue utilizada para calcular los cuantiles empíricos de las CDF. Dos medidas cuantitativas fueron utilizadas para comparar las CDFs: la primera de ellas es la métrica propuesta por Perkins et al. (2007), que mide el área común bajo dos curvas PDF ( $S_{S C O R E}$; utilizada en Giraldo Osorio and García Galiano, 2011); la otra es el valor-p del test de bondad de ajuste Smirnov-Kolmogorov para dos muestras (two sample Smirnov-Kolmogorov goodness of fit test, TSSK; utilizado en García Galiano and Giraldo Osorio, 2011).

Para la estimación del criterio de desempeño $R_{B}$, las CDF empíricas fueron construidas para los datos observados y desde RCMs en el periodo 1961-1990. La PDF de los datos observados es la de referencia para este periodo. Para el criterio de convergencia $R_{D}$, la dificultad radica en que se desconoce la PDF de referencia en el futuro, por lo que debe ser estimada mediante un proceso iterativo (Giorgi and Mearns, 2002). Debe advertirse que la PDF construida de esta manera es sólo un estimado de la distribución a futuro. De acuerdo con Giorgi and Mearns (2002, 2003), el promedio REA no representa la respuesta real del clima a los escenarios de forzamiento, sin embargo, es el mejor estimado de esta respuesta. 
Finalmente, la probabilidad asociada con el cambio simulado por el RCM es proporcional a su factor $R$ (Giorgi and Mearns, 2002, 2003). El factor normalizado $P m$, en la ecuación (2), se puede interpretar como la probabilidad asociada con cada RCM. Se ha demostrado que los factores normalizados son análogos a los factores de precisión definidos en aproximaciones bayesianas (Tebaldi et al., 2005; Tebaldi and Knutti, 2007). La probabilidad $P m$ del $\mathrm{RCM}_{\mathrm{i}}$ se define como (Giorgi and Mearns, 2003):

$$
P m_{i}=\frac{R_{i}}{\sum_{j=1}^{N} R_{j}} \quad i=1,2, \ldots, N
$$

donde $N$ es el número de modelos considerados para construir el ensemble.

\subsubsection{Métricas utilizadas para calcular los factores de ponderación}

El test TSSK es un test no paramétrico que evalúa la concordancia entre las distribuciones de probabilidad de dos muestras independientes. El test cuantifica la distancia máxima entre las CDF empíricas construidas desde las series. El estadístico de dos colas es el siguiente:

$$
D_{n_{1}, n_{2}}=\max _{x}\left|S_{n_{1}}(y)-S_{n_{2}}(y)\right|
$$

donde $S_{n 1}(y)$ y $S_{n 2}(y)$ son las distribuciones empíricas de las muestras independientes, $n_{1}$ y $n_{2}$ corresponden a los tamaños de las muestras. Para la distribución asintótica de la distancia de Kolmogorov se ha probado (Gibbons and Chakraborti, 2003) como:

$$
\begin{aligned}
& \lim _{n_{1}, n_{2} \rightarrow \infty} P\left[\sqrt{\frac{n_{1} n_{2}}{n_{1}+n_{2}}} D_{n_{1}, n_{2}} \leq d\right]=L(d) \\
& \text { con } L(d)=1-2 \sum_{i=1}^{\infty}(-1)^{i-1} \exp \left(-2 i^{2} d^{2}\right)
\end{aligned}
$$

Finalmente, el valor-p del test de bondad de ajuste se calcula con la siguiente ecuación:

$$
p(d)=1-L(d)=2 \sum_{i=1}^{\infty}(-1)^{i-1} \exp \left(-2 i^{2} d^{2}\right)
$$

Como se ha comentado, Perkins et al. (2007) desarrollaron un método para evaluar la similaridad entre dos PDF a través del denominado skill score $\left(S_{S C O R E}\right)$, que calcula el área común bajo las dos curvas definidas por las PDF, como se presenta en la Figura 
6. Si las distribuciones coinciden de manera perfecta, el $S_{S C O R E}$ sería igual a la unidad. Como la relación entre las CDF empíricas (usadas por el test TSSK) y las PDF empíricas es directa, el $S_{S C O R E}$ es fácil de calcular. Su definición en el caso continuo, sería la siguiente:

$$
S_{\text {SCORE }}=\int_{-\infty}^{x_{0}} f_{1}(x) d x+\int_{x_{0}}^{+\infty} f_{2}(x) d x
$$

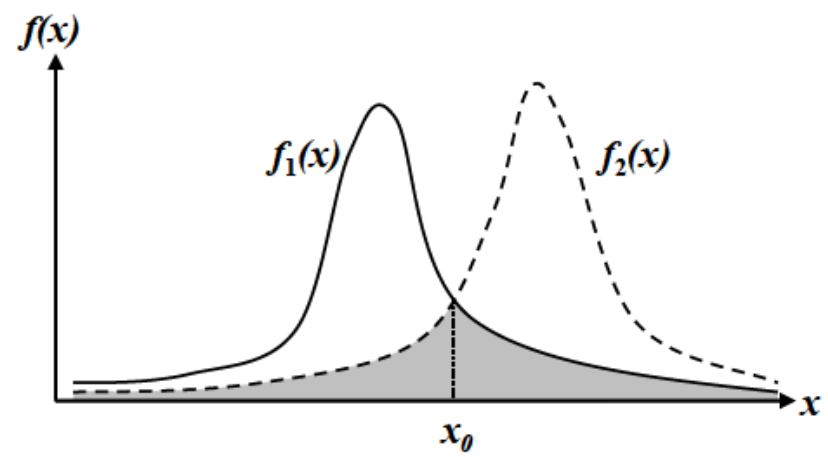

Figura 6. Métrica definida por Perkins et al. (2007). El $S_{S C O R E}$ es la medida del área bajo ambas curvas $f_{1}(x)$ y $f_{2}(x)$ (área sombreada).

\subsection{Riesgo de Eventos Extremos}

La identificación de umbrales para la definición de las sequías y las precipitaciones extremas, así como la evidencia sobre el cambio en la frecuencia de estos eventos, sentará las bases para los estudios de impacto (inundaciones, erosión del suelo, deslizamientos, degradación ambiental, etc.). Varios trabajos anteriores han abordado el tema del cambio en la frecuencia de los eventos extremos de sequía y precipitación en diferentes regiones del planeta, con datos observados o desde modelos climáticos (Goswami et al., 2006; Rajeevan et al., 2008). Mapas del cambio o del riesgo de eventos de sequía fueron construidos por Blenkinsop and Fowler (2007) para Reino Unido, y Sánchez et al. (2011) para la Península Ibérica. Por otra parte, Groisman et al. (2005) calcularon los cambios observados en las precipitaciones más intensas, y analizaron su tendencia desde tres GCMs, llegando a la conclusión que aumentará la frecuencia de los eventos más intensos de precipitación en muchas regiones extratropicales. Su trabajo fue muy valioso, aunque reconocían que estimaciones confiables sobre los cambios observados y su relación con las tendencias pronosticadas por los GCM, son posibles sólo en regiones con densos sistemas de medición, debido al pequeño radio de correlación espacial entre los eventos más intensos. 
En esta Tesis se realizaron análisis regionales de las rachas secas, considerando las ecorregiones al interior de la cuenca y que fueron definidas por Olson et al. (2001). Para analizar las precipitaciones máximas se construyeron mapas interpolados de diferentes estadísticos, calculados desde las PDF ensemble construidas en cada sitio, dada su intermitencia espacial.

Los valores estadísticos interpolados en la zona de estudio fueron la media y la desviación estándar de las AMDR, junto con sus intervalos de confianza. Además de estos estadísticos básicos de las AMDR, se construyeron mapas para diferentes cuantiles de la distribución (5, 10, 25, 50, 75, 90 y 95\%), asociados a diferentes periodos de retorno. Calculando la diferencia entre los mapas para dos años de referencia (1990 como año de referencia del cambio, y 2050 como horizonte de pronóstico), se identificaron las tendencias espacio-temporales de las diferentes variables estudiadas.

En este sentido, un claro aporte del presente trabajo a la construcción de capacidad adaptativa son los mapas de riesgo de eventos de precipitación extrema, y los análisis regionales probabilísticos del cambio en las rachas secas para la Cuenca del Río Senegal. Del análisis de las precipitaciones extremas, se identificó que el Valle del Río Senegal presentará aumento de las precipitaciones máximas, en una zona muy vulnerable a las inundaciones. Luego, el contar con tecnologías y herramientas para el seguimiento en tiempo cercano al real de eventos altamente dinámicos como las inundaciones, constituye un paso más en el esfuerzo de construir capacidad adaptativa. En este sentido, la teledetección tiene un papel importante.

\subsection{Teledetección Aplicada a la Detección de Inundaciones}

Es evidente que la delineación precisa de las inundaciones permite detectar dificultades en las medidas para el control de la creciente, organizar la distribución de las ayudas en las zonas más afectadas, y una vez la inundación se haya retirado, calcular las reclamaciones por daños (Smith, 1997).

Sin embargo, los métodos tradicionales para medir las variables hidrológicas con frecuencia fallan para registrar un evento de inundación extremo. Debido a lo anterior, las técnicas basadas en sensores remotos y teledetección se han convertido en una opción confiable y económica para cartografiar y calcular los daños de las inundaciones, dada la escasez y deficiencia de las redes de medición en los países en vías de desarrollo (Sanyal and Lu, 2004; Khan et al., 2011).

En este marco, las imágenes de satélite se han convertido en herramientas clave para el seguimiento de las inundaciones, ya que proveen de una cobertura sinóptica y 
sistemática de los fenómenos naturales sobre la superficie terrestre (Zhan et al., 2000, 2002; Tralli et al., 2005; Khan et al., 2011). Los SIG y la teledetección, se han convertido en herramientas clave para llevar a cabo análisis regionales de planicies de inundación, realizando un seguimiento de la evolución geomorfológica y de los cambios en el uso del suelo (Andersen et al., 2001; Sanyal and Lu, 2004).

Para el seguimiento de eventos naturales dinámicos como las inundaciones, no existe un único dispositivo apropiado para todas las situaciones. Los sensores remotos ópticos son incapaces de escanear la superficie terrestre en condiciones de nubosidad (Smith, 1997; Brivio et al., 2002; Wang et al., 2002). Por otra parte, las olas superficiales del agua y la vegetación emergente incrementan la rugosidad, lo que complica la delineación del área inundada con sensores activos de microondas (Smith, 1997; Yang et al., 1999; Wang, 2002; Benger, 2003; Sanyal and Lu, 2004; Martínez and Le Toan, 2007). Con el fin de superar estos problemas, se han desarrollado nuevas metodologías que emplean datos desde diversas fuentes para detectar las áreas inundadas. El uso de imágenes desde diferentes plataformas y tecnologías, en conjunto con mapas de uso del suelo e información demográfica, permite preparar análisis del riesgo de inundación de asentamientos humanos y calcular la vulnerabilidad de las infraestructuras (Profeti and Macintosh, 1997; Kim and Chi, 1998; Wang, 2004; Sakamoto et al., 2005; Sanyal and Lu, 2005; Töyráä and Pietroniro, 2005). Distintos autores (Giacomelli et al., 1997; Towsend and Walsh, 1998; Saich et al., 2001; Brivio et al., 2002; Benger, 2003), han demostrado la utilidad potencial de los Modelos de Elevación Digital (DEM) en conjunto con las imágenes de satélite, para la cartografía de zonas inundadas.

Particularmente, el seguimiento de un evento de inundación requiere del uso de imágenes de satélite con una alta resolución temporal. Sin embargo, la resolución espacial de estas imágenes no es siempre apropiada, lo que entorpece la delineación correcta de las áreas inundadas. En consecuencia, está justificado el desarrollo de una metodología a nivel de sub-píxeles (o sub-celdas). Este Análisis de Sub-píxeles (SA) debe tener en cuenta el DEM de la zona de estudio, con el fin de identificar apropiadamente la zona inundada en las zonas bajas del terreno.

El método SA comienza con el Modelo de Mezcla Lineal (linear mixture model, LMM) para calcular las fracciones de cobertura dentro de los píxeles (celdas) gruesos. Luego, se utiliza la dependencia espacial de estas fracciones entre píxeles gruesos para ubicar los sub-píxeles de las diferentes clases, utilizando el DEM y apoyándose en dos hipótesis: (1) los sub-píxeles tienden a estar agrupados alrededor del centroide 
de la clase de cobertura y, (2) los sub-píxeles de la clase "agua" (o clase "inundado") tienden a localizarse en las partes bajas del terreno.

Posteriormente, nuevas áreas inundadas son identificadas usando las trayectorias de drenaje, definidas desde el DEM. Concluyendo, una metodología innovadora ha sido desarrollada para incorporar un algoritmo DOD (drainage on DEM) modificado, ajustado a los requerimientos del estudio. Las salidas del procedimiento original propuesto por Giacomelli et al., (1997) eran sensibles a consideraciones subjetivas una vez que requería la intervención del usuario para seleccionar el número umbral de trayectorias que atravesaban una celda. El algoritmo DOD modificado utiliza el concepto de agregar las celdas en la dirección de las trayectorias del drenaje, evitando ser afectado por la subjetividad del usuario. Finalmente, los clúster de pocos píxeles aislados son identificados y reclasificados, utilizando el procedimiento $\mathrm{ICl}$ (Isolated Cluster Identification) modificado, basado en el trabajo de Jianghong et al. (2004). En contraste con el trabajo de Jianghong et al. (2004), donde los cluster aislados eran clasificados en una única clase vecina, el nuevo procedimiento permite reclasificar las celdas del clúster en más de una única clase de cobertura, usando las coberturas vecinas como criterio de clasificación.

La metodología de SA, en conjunto con el DEM de la zona de estudio, mejora la identificación de las áreas inundadas. El desarrollo de la metodología fue motivado por la naturaleza altamente dinámica de los eventos de inundación, que impone como condición a su seguimiento observaciones con gran cobertura espacial, pero al mismo tiempo con una resolución adecuada. El desarrollo de la metodología de SA para la detección de inundaciones (Giraldo Osorio and García Galiano, 2012) es otro aporte claro para el desarrollo de medidas que mejoren la capacidad adaptativa, ya que se podrá disponer de mapas de la inundación en tiempo cercano al real, con adecuada resolución espacial. 



\section{PUBLICACIONES QUE CONSTITUYEN LA TESIS}

\subsection{Resumen del Artículo 1}

\subsubsection{Título}

Analysis of impacts on hydrometeorological extremes in the Senegal River Basin from REMO RCM.

\subsubsection{Objetivos específicos}

- Evaluar la idoneidad del RCM REMO desarrollado por el Max-Planck Institute for Meteorology (Alemania), utilizado en la simulación de variables relacionadas con el patrón espacial de la precipitación: ciclo estacional de la precipitación, longitud de las rachas secas y número de eventos extremos de precipitación.

- Analizar el cambio en los cuantiles de la distribución empírica de la longitud de rachas secas.

- Analizar las tendencias en el número de eventos de precipitación, considerando eventos de diferente magnitud (moderada, fuerte y muy fuerte).

\subsubsection{Metodología}

El estudio ha sido enfocado en la Cuenca del Río Senegal $\left(10^{\circ} \mathrm{N}-18^{\circ} \mathrm{N} ; 18^{\circ} \mathrm{W}-6^{\circ} \mathrm{W}\right)$. La cuenca se caracteriza por un patrón irregular de lluvias con un claro gradiente latitudinal, decreciente hacia el norte.

Se han utilizado datos modelados de precipitación diaria desde tres ejecuciones del RCM REMO (Jacob, 2001; Paeth et al., 2005), con una resolución espacial de 0.5을 en el marco del Proyecto Europeo AMMA (Polcher et al., 2011). Los datos observados han correspondido con mallas de precipitación mensual del CRU (Climate Research Unit, University of East Anglia; New et al., 2000), con una resolución espacial de 0.5은 y mallas a nivel diario del IRD, con resolución espacial de $1 \stackrel{0}{\text {. }}$

Se utilizaron los datos mensuales (CRU y REMO) para el análisis del patrón espacial de la precipitación y su ciclo estacional. La manera más simple de representar la divergencia entre ambas bases de datos, consiste en elaborar mapas de la diferencia de la precipitación media calculada para las mallas observados (CRU) y las simuladas (REMO). También se puede calcular el valor de la significancia para una prueba de hipótesis para la media, suponiendo varianzas distintas.

Se seleccionaron algunos sitios específicos para realizar el análisis del ciclo anual de la precipitación, teniendo en cuenta la disponibilidad de datos del IRD. Utilizando los promedios mensuales multianuales (observados y simulados), se calculó la raíz del 
error cuadrático medio (root mean square error-RMSE-), y su proporción respecto del valor medio de la precipitación observada.

Para el análisis de la longitud de las rachas secas (dry spell length -DSL-), se consideró la temporada monzónica entre el 1 de mayo y el 30 de septiembre. Se definieron las DSL como el número de días consecutivos con una cantidad de precipitación por debajo de un umbral ( $1 \mathrm{~mm} /$ día ó $10 \mathrm{~mm} /$ día $)$ en el periodo monzónico. Aún cuando las rachas más largas podrían abarcar varios años (especialmente para el umbral de $10 \mathrm{~mm}$ ), la definición restringe los días que aportan a la longitud de la racha a los que efectivamente pertenecen a la estación monzónica. De esta manera, se obtuvieron series temporales de DSL desde los datos IRD y REMO. La discontinuidad espacial de los datos IRD restringió los análisis a los sitios donde existe información de esta base de datos (43 celdas en la región). Además, los datos IRD también restringieron la ventana temporal (de 01/01/1970 a 31/12/1990). En todos los sitios seleccionados, se construyeron las funciones de distribución acumulada (CDF) empíricas, para las series temporales observadas y simuladas. Luego, el análisis del desvío fue abordado calculando el p-valor de la prueba de bondad de ajuste Smirnov-Kolmogorov para dos muestras.

Para el análisis del cambio futuro de las rachas secas, se construyeron las distribuciones empíricas de las DSL a partir de los datos REMO en los sitios seleccionados. Las series se dividieron en tres periodos, periodo de referencia 19611990, y dos horizontes de pronóstico 1991-2020 y 2021-2050, y en cada periodo se construyó la CDF empírica, junto con el intervalo de confianza al 95\% para cada percentil, utilizando bootstrapping.

Para el análisis de los eventos lluviosos a nivel diario, la cuenca se dividió en dos zonas de análisis, de acuerdo con las características de la precipitación. Se consideraron diferentes tipos de eventos lluviosos diarios (eventos moderados, eventos fuertes, y eventos muy fuertes), y se cuantificaron estos eventos para los datos REMO en las mismas ventanas o periodos temporales utilizados anteriormente. Además, se cuantificó el desvío contrastando el número de eventos contabilizados para cada RCM con los datos IRD en el periodo 1970-1990.

Promediando las tres ejecuciones REMO (o ensemble members), se construyeron mapas del número de eventos en cada ventana temporal. Desde estos mapas se obtuvieron las distribuciones empíricas para cada ventana temporal, para cada tipología de los eventos, en cada una de las zonas de la cuenca, que fueron comparadas utilizando box-plots. Además, dentro de cada periodo se analizó la 
tendencia de las series temporales utilizando el test no paramétrico de Mann-Kendall (Kendall, 1975).

\subsubsection{Resultados}

La Figura 7 presenta los mapas de significancia de la prueba de hipótesis para las precipitaciones medias en el periodo de referencia (resultados mencionados, pero no visualizados en el artículo). De acuerdo con estos mapas, la base de datos REMO utilizada en este trabajo muestra una simulación confiable de la precipitación en la mayor parte de la Cuenca del Río Senegal (zonas del Sahel y Sudaniana). Sin embargo, y de acuerdo con el análisis del ciclo estacional de la precipitación, ésta es subestimada en toda la cuenca, especialmente en la zona del Sahel.

El análisis del desvío de las rachas secas mostró que las DSL están mejor modeladas, por las ejecuciones REMO, para el umbral de $1 \mathrm{~mm} /$ día. Para ambos umbrales de precipitación, las DSL están mejor modeladas por REMO en la mitad Sur de la cuenca, en la ecorregión Sudaniana.

El análisis del cambio en las DSL arrojó como evidencia el aumento de la longitud de las rachas secas en los horizontes de pronóstico, respecto al periodo de referencia. Sin embargo, el cambio de la distribución no es significativo en todos los casos. Para los sitios de análisis seleccionados, el umbral de $10 \mathrm{~mm} /$ día presentó aumentos significativos en 2021-2050, pero ello no se cumple para todos los sitios en el periodo 1991-2020. Por otra parte, en el caso del umbral $1 \mathrm{~mm} /$ día, se presentaron aumentos significativos sólo en tres sitios seleccionados para el horizonte 2021-2050.

El análisis de los eventos lluviosos se ha justificado al observar el desplazamiento hacia el Sur de las isolíneas en los mapas de porcentaje de días con precipitaciones mayores que $10 \mathrm{~mm} /$ día. Desde el análisis del desvío de los diferentes tipos de eventos considerados (moderados, fuertes y muy fuertes), se ha concluido que el RCM REMO sobrestima el número de eventos muy fuertes, mientras que el número de eventos moderados es subestimado. A pesar de que el desvío con los datos observados era evidente, se continúo con el análisis de las tendencias en el número de eventos. Los box-plots construidos para cada evento, en cada zona de la cuenca considerada, revelan un descenso del número de eventos para el periodo 2021-2050, especialmente para los eventos moderados y fuertes. Por otra parte, el análisis aplicando el Test de Mann-Kendall reveló que, en general, no existen tendencias significativas en los periodos considerados, mostrando la necesidad de utilizar otras herramientas para el análisis no estacionario de las variables hidrometeorológicas. 

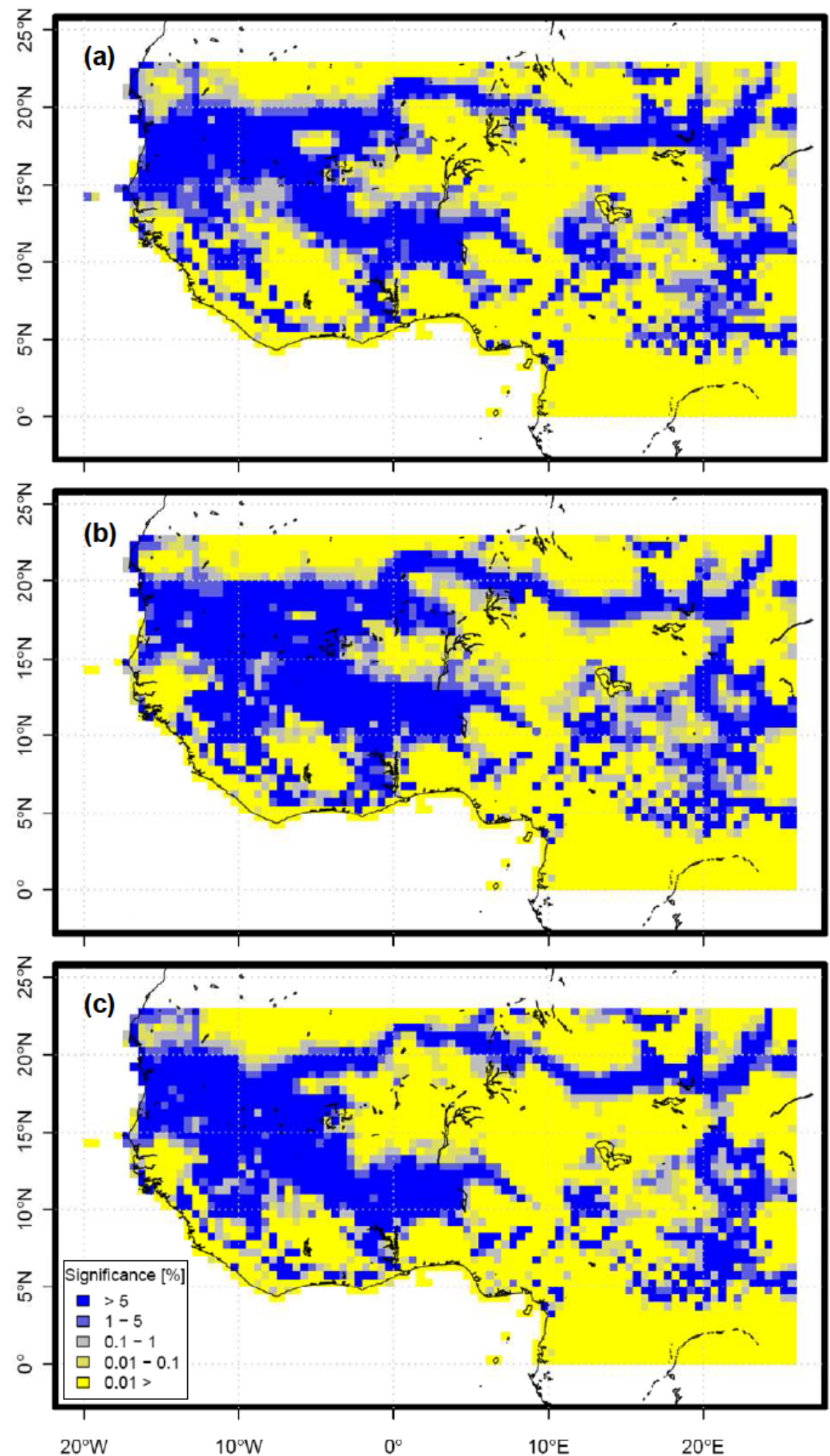

Figura 7. Valor de la significancia de la prueba de hipótesis para la igualdad de la media de la precipitación anual (calculada con CRU y REMO) en el periodo 1961-1990 para África Occidental. (a) REMO-901, (b) REMO-902 y (c) REMO-903. 


\subsubsection{Conclusiones y recomendaciones}

El presente trabajo ha demostrado que las zonas del Sahel y Sudaniana, al interior de la cuenca del Río Senegal, exhiben patrones climáticos diferentes, que están relacionados con el forzamiento que ejerce el WAM sobre el clima del África Occidental, y con el potencial efecto sobre el patrón de variabilidad espacial de la precipitación debido a un cambio de las condiciones superficiales (cambio en el uso del suelo, degradación ambiental debido a la tala de zonas boscosas para la agricultura y el pastoreo, etc.).

El conocimiento de la distribución espacial de los eventos extremos de sequía y sus estadísticos es fundamental para la gestión de los recursos hídricos a escala de cuenca. Los análisis realizados a las distribuciones empíricas de las DSL, han revelado un incremento de su longitud, especialmente en la zona del Sahel. Los análisis han concluido, además, que la diferencia será mayor para el periodo 20212050.

Se utilizó un único RCM (REMO), con varias ejecuciones del mismo, constituyendo un ensemble de física transformada (perturbed physics ensemble, PPE, Tebaldi and Knutti, 2007). El análisis del desvío demostró la adecuada capacidad de REMO para modelar DSL monzónicas. De acuerdo con los resultados obtenidos, las diferentes ejecuciones del RCM presentan un buen desempeño en toda la zona de estudio al calcular los cuantiles empíricos para el umbral de $1 \mathrm{~mm} /$ día. Sin embargo, para el umbral $10 \mathrm{~mm} /$ día los mejores resultados se observaron en la zona Sudaniana de la cuenca, al sur de la zona de estudio.

De acuerdo con las proyecciones del RCM REMO, se espera un incremento de los cuantiles más altos de la distribución de las DSL para $1 \mathrm{~mm} /$ día, especialmente en la zona del Sahel para el periodo 2021-2050. Sin embargo, el aumento no es significativo para el periodo 1991-2020. Para el umbral $10 \mathrm{~mm} /$ día el aumento en los cuantiles más altos de la distribución será significativo para toda la cuenca en el periodo 2021-2050, sin embargo, no será significativo en 1991-2020.

En general, el RCM REMO subestimó el número de eventos moderados, y sobreestimó los eventos muy fuertes. En el periodo 2021-2050, la tendencia de los eventos moderados es a disminuir, mientras que los eventos muy fuertes tienden a aumentar. Sin embargo, ambas tendencias no son significativas.

Se concluye que, en general, los RCM no representan adecuadamente la precipitación. Debido a ello, futuros trabajos deberían incluir los RCM publicados por el proyecto europeo ENSEMBLES (Christensen et al., 2009) en la creación de mapas de riesgo de eventos climáticos extremos. Además, debido a la fuerte no estacionariedad 
de las series analizadas, se recomienda la consideración de otras metodologías para el análisis de las tendencias de las series temporales. 


\title{
5.1.6 Copia de la publicación
}

\section{Analysis of impacts on hydrometeorological extremes in the Senegal River Basin from REMO RCM}

\author{
SANDRA García Galiano * and JUAN DiEgo Giraldo Osorio \\ Technical University of Cartagena, Department of Thermal Engineering and Fluids, Cartagena, Spain \\ (Manuscript received October 31, 2009; in revised form May 6, 2010, accepted May 6, 2010)
}

\begin{abstract}
West Africa is highly vulnerable to climate variability. The precipitation latitudinal gradient determines agricultural activities. The cultivated area of the Sahel is a densely populated region, whereas flood recession agriculture is practiced in the Senegal River Valley. The present study analyses both spatial-temporal rainfall patterns of the REMO Regional Climate Model (RCM) and observed rainfall data, focusing in particular on extreme hydro-meteorological phenomena. An analysis of simulated daily rainfall data was performed to determine the frequency and magnitude of length of dry spells, as well as the extreme rainfall events. A projected annual decrease in rainfall on horizon 2050 could be explained by two factors: the decrease in the percentage of rainy days on both west and north sides of the basin, and the decrease of precipitation amount for rainy days in the southem basin. Finally, an increase in the frequency of dry spell in the monsoon season by 2050 is projected. Such findings are significant in a framework of strategies for water resources management and planning at basin scale, in order to build adaptive capacity.

Zusammenfassung

West Afrika ist sehr empfindlich gegen Klimavariabilität. Der Höhengradient des Niederschlags beeinflusst landwirtschaftliche Aktivitäten. Das kultivierte Gebiet vom Sahel ist eine dicht besiedelte Fläche, wobei landwirtschaftliche Aktivitäten. Das kultivierte Gebiet vom Sahel ist eine dicht besiedelte Fläche, wobei
Überschwemmungsfeldbau im Flußtal des Senegal praktisiert wird. Diese Forschung analysiert sowohl die räumlich-zeitlichen Niederschlagsmuster des REMO Regionalen Klima-Modells (RCM) als auch die die räumlich-zeitlichen Niederschlagsmuster des REMO Regionalen Klima-Modells (RCM) als auch die beobachteten Niederschlagsdaten, insbesondere von extremen Hydrometeorologischen Phänomenen. Eine Analyse täglichen Niederschlags wurde ausgeführt, um Häufigkeit und Länge der Dürreperioden zu bestimmen, sowie extreme Niederschlagsereignisse. Eine vorhergesagte jährige Abnahme an Niederschlag um 2050 könnte durch zwei Faktoren erklärt werden: die Abnahme im Prozentsatz regnerischer Tage im Westen und Norden des Flussbeckens, und die Abnahme an Niederschlag an regnerischen Tagen im südlichen Teil. Schließlich wird eine Zunahme der Häufigkeit von Dürreperioden in der Monsunsaison um 2050 vorhergesagt. Solche Ergebnisse sind von Bedeutung in einem Rahmen von Strategien für Flussbeckenmanagement
\end{abstract} und -planung, um Anpassungsfähigkeit zu bauen.

\section{Introduction}

The complexity of West African Monsoon (WAM) dynamics and their connection with rainfall variability were briefly explained by several authors (DIEDHOU et al., 1999; DIEDHOU et al., 2001; PAETH et al., 2005; LEBEL and ALI, 2009). The Intertropical Convergence Zone (ITCZ) is the main factor controlling the rainfall over West Africa on a large scale. However, the interannual rainfall variability in the Sahel is determined by the African Easterly Jet (AEJ), on a regional scale (LEBEL and ALI, 2009; GRIST and NICHOLSON, 2001). Several factors interact not only in the generation of the AEJ but also in the African Easterly Waves (AEWs), and the WAM. These factors include sea surface temperatures (SST), land changes, large scale tropical phenomena such as El Niño-Southern Oscillation (ENSO) and extratropical circulations such as North Atlantic Oscillation (PAETH et al., 2005). In any case, WAM, AEJ,

*Corresponding author: Sandra García Galiano, Technical University of Cartagena, Paseo Alfonso XIII, 52, 30203 Cartagena. Spain, e-mail: sandra.garcia@upct.es
AEW and ITCZ should be understood as one system with many interconnections. Development of strategies is very important in order to build adaptive capacity for climate risks management. According to the Intergovernmental Panel on Climate Change (IPCC), "adaptive capacity" can be understood as the "ability to cope, adapt or recover from the effects of a hazard", or in this case, to climate change. Adaptive capacity is considered a necessary condition for designing and implementing effective adaptation strategies, and could be achieved by increasing the knowledge of potential climate risks in individual river basins (EC, 2009).

Therefore, studies focused on the analysis of spatial rainfall patterns and trends in extreme hydrometeorological events at basin scale - more specifically, in the Senegal River basin - from Regional Climate Models (RCMs) are a breakthrough in this field. The present work is based on a specific RCM and observed daily gridded rainfall. An analysis of rainfall spatialtemporal patterns in the basin for the whole period $1960-2050$ is examined (also 1991-2020). The rainy season is characterized by the number and intensity of daily rainfall events. 

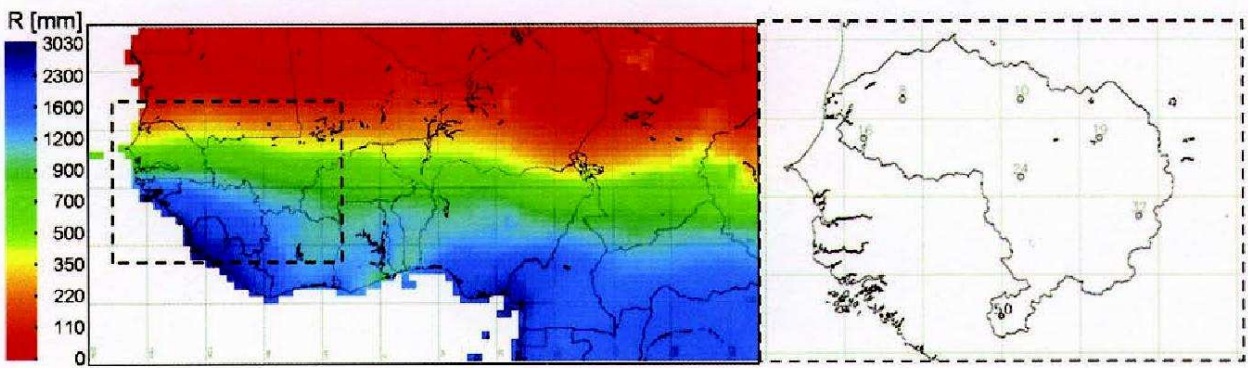

Figure 1: Mean annual rainfall R (mm) from CRU data (1961-1990 period), Senegal River basin and locations for impacts studies.

Table 1: RMSE for the studied locations: (a) RMSE between REMO ensemble members and CRU data, and (b) Ratio between the accumulated RMSE and the mean annual rainfall from CRU data $\left(\mathrm{R}_{C R U}\right)$.

\begin{tabular}{|c|c|c|c|c|c|c|c|c|c|}
\hline \multirow{2}{*}{ Loc. } & \multirow{2}{*}{$\begin{array}{c}R_{\text {CRU }} \\
\text { [mm/year] }\end{array}$} & \multicolumn{4}{|c|}{ (a) RMSE [mm] } & \multicolumn{4}{|c|}{ (b) RMSE/R RRU } \\
\hline & & REMO-901 & REMO -902 & REMO-903 & REMO $_{\text {AVER }}$ & REMO-901 & REMO-90 2 & REMO-903 & REM OAVER \\
\hline [08] & 294.7 & 53.84 & 60.44 & 57.67 & 56.40 & 0.18 & 0.21 & 0.20 & 0.19 \\
\hline$[10]$ & 329.7 & 61.55 & 46.52 & 45.83 & 49.62 & 0.19 & 0.14 & 0.14 & 0.15 \\
\hline [16] & 442.1 & 62.07 & 73.65 & 67.94 & 65.73 & 0.14 & 0.17 & 0.15 & 0.15 \\
\hline [19] & 449.6 & 51.88 & 49.90 & 57.73 & 49.50 & 0.12 & 0.11 & 0.13 & 0.11 \\
\hline [24] & 694.6 & 80.71 & 87.73 & 98.77 & 85.02 & 0.12 & 0.13 & 0.14 & 0.12 \\
\hline [32] & 805.6 & 72.91 & 78.95 & 77.34 & 71.29 & 0.09 & 0.10 & 0.10 & 0.09 \\
\hline
\end{tabular}

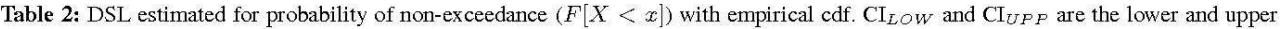
confidence interval limits of 1961-1990 cdf, respectively.

\begin{tabular}{|c|c|c|c|c|c|c|}
\hline \multicolumn{7}{|c|}{ Threshold $=1 \mathrm{~mm} / \mathrm{day}$} \\
\hline \multicolumn{2}{|c|}{ Loc. $F[X<x]$} & \multicolumn{3}{|c|}{$\begin{array}{c}1961-1990 \\
\mathrm{Cl}_{\text {Low }} \mathrm{Cl}_{\text {upp }} \\
\end{array}$} & \multirow{3}{*}{$\begin{array}{r}\mathbf{1 9 9 1 -} \\
\mathbf{2 0 2 0} \\
6.0\end{array}$} & \multirow{3}{*}{$\begin{array}{c}\mathbf{2 0 2 1}- \\
\mathbf{2 0 5 0} \\
6.4 \\
11.4\end{array}$} \\
\hline [08] & 0.50 & 4.0 & 4.0 & 4.2 & & \\
\hline & 0.75 & 8.9 & 7.1 & 10.7 & & \\
\hline & 0.90 & 20.5 & 14.9 & 27.9 & 25.7 & 31.3 \\
\hline & 0.95 & 36.3 & 26.0 & 46.2 & 39.6 & 46.3 \\
\hline \multirow[t]{4}{*}{ [10] } & 0.50 & 4.0 & 4.0 & 4.0 & 4.0 & 5.0 \\
\hline & 0.75 & 7.7 & 6.3 & 9.2 & 8.8 & 10.1 \\
\hline & 0.90 & 15.9 & 12.6 & 22.8 & 17.4 & 22.9 \\
\hline & 0.95 & 29.1 & 20.0 & 39.0 & 34.2 & 40.3 \\
\hline \multirow{4}{*}{ [19] } & 0.50 & 2.0 & 2.0 & 2.4 & 3.0 & 3.0 \\
\hline & 0.75 & 4.3 & 3.6 & 5.1 & 4.7 & 5.9 \\
\hline & 0.90 & 9.6 & 7.1 & 12.5 & 11.0 & 12.2 \\
\hline & 0.95 & 17.2 & 11.4 & 23.8 & 19.8 & 24.9 \\
\hline \multirow[t]{4}{*}{ [24] } & 0.50 & 2.0 & 2.0 & 2.0 & 3.0 & 3.0 \\
\hline & 0.75 & 3.7 & 3.0 & 4.5 & 4.4 & 4.7 \\
\hline & 0.90 & 8.8 & 6.4 & 13.4 & 9.8 & 10.4 \\
\hline & 0.95 & 17.2 & 11.3 & 20.7 & 18.3 & 21.7 \\
\hline \multirow[t]{4}{*}{ [32] } & 0.50 & 2.0 & 2.0 & 2.0 & 3.0 & 3.0 \\
\hline & 0.75 & 3.0 & 2.5 & 3.8 & 3.9 & 3.9 \\
\hline & 0.90 & 7.0 & 5.5 & 8.8 & 8.0 & 8.0 \\
\hline & 0.95 & 10.5 & 82 & 14.0 & 11.9 & 12.9 \\
\hline \multirow[t]{4}{*}{ [50] } & 0.50 & 2.0 & 1.9 & 2.0 & 2.0 & 2.0 \\
\hline & 0.75 & 3.1 & 2.4 & 3.8 & 3.3 & 3.1 \\
\hline & 0.90 & 6.9 & 5.7 & 9.4 & 8.3 & 7.1 \\
\hline & 0.95 & 10.5 & 8.6 & 11.5 & 12.1 & 13.9 \\
\hline
\end{tabular}

\begin{tabular}{ccc|c|c}
\multicolumn{6}{c}{ Thresh old $=10 \mathrm{~mm} /$ day } \\
\hline \hline \multicolumn{3}{c|}{ 1961-1990 } & $\mathbf{1 9 9 1 -}$ & $\mathbf{2 0 2 1 -}$ \\
CILOW & Cl UpP & $\mathbf{2 0 2 0}$ & $\mathbf{2 0 5 0}$ \\
\hline \hline 25.0 & 25.0 & 28.7 & 25.0 & 26.4 \\
81.9 & 69.3 & 99.0 & 84.3 & 108.2 \\
124.9 & 115.5 & 133.9 & 136.6 & 160.3 \\
139.0 & 131.5 & 149.0 & 151.9 & 236.9 \\
\hline 19.0 & 19.0 & 23.7 & 26.6 & 39.7 \\
58.9 & 42.8 & 75.4 & 68.4 & 112.2 \\
116.1 & 100.0 & 125.0 & 121.3 & 146.5 \\
137.6 & 123.5 & 146.8 & 142.0 & 177.0 \\
\hline 11.0 & 11.0 & 11.3 & 12.0 & 22.0 \\
24.0 & 20.2 & 29.3 & 30.3 & 38.7 \\
65.7 & 45.4 & 80.0 & 78.3 & 93.1 \\
85.8 & 78.0 & 102.0 & 96.2 & 114.8 \\
\hline 8.0 & 8.0 & 8.1 & 9.0 & 11.8 \\
14.4 & 12.6 & 17.5 & 17.0 & 24.6 \\
34.9 & 26.5 & 51.9 & 46.7 & 63.2 \\
66.7 & 49.8 & 73.1 & 69.0 & 84.8 \\
\hline 4.0 & 4.0 & 4.6 & 5.3 & 6.0 \\
9.1 & 7.7 & 11.3 & 11.2 & 12.5 \\
23.3 & 15.0 & 31.7 & 27.0 & 31.7 \\
39.2 & 28.6 & 53.0 & 46.7 & 54.7 \\
\hline 2.1 & 2.0 & 2.6 & 3.0 & 3.0 \\
4.7 & 4.0 & 5.6 & 5.4 & 5.0 \\
9.5 & 7.7 & 12.3 & 10.0 & 10.6 \\
16.6 & 11.3 & 25.4 & 20.1 & 20.8 \\
\hline \hline \multicolumn{5}{|c}{}
\end{tabular}



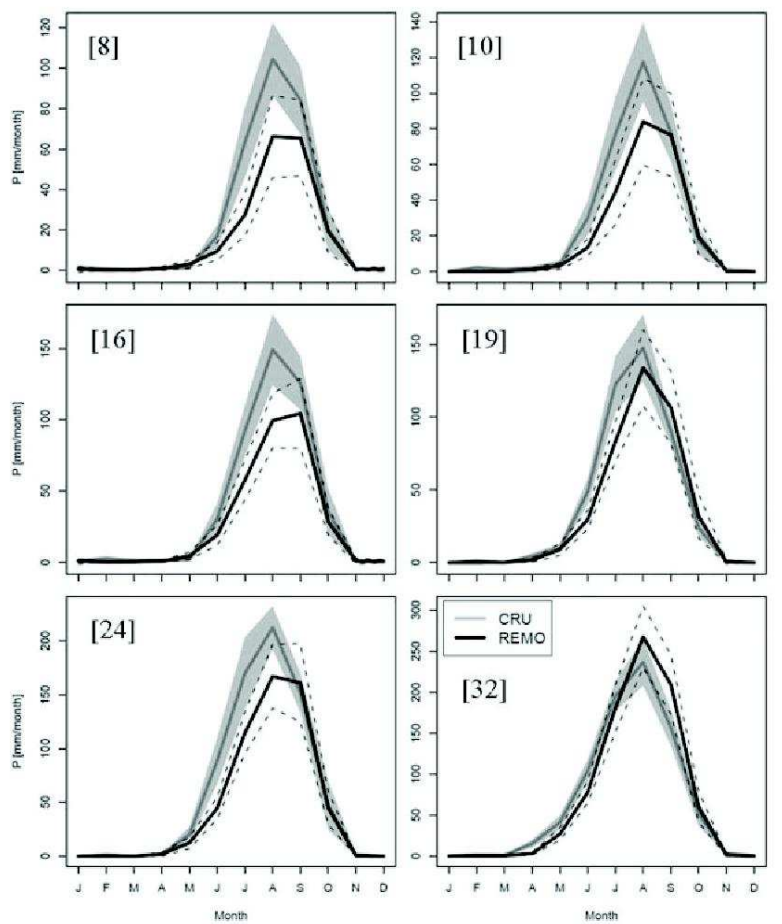

Figure 2: Analysis of seasonal rainfall cycle (1961-1990 period) in selected locations. Mean monthly rainfall comparison between an average over the three ensemble REMO members and CRU data. The grey area shows the CI of CRU data, the dashed lines are the CI of REMO data, and the ordinates are automatically fitted for each panel.

\section{Study area and datasets}

The study was focused on the Senegal River Basin, located between $10^{\circ} \mathrm{N}-18^{\circ} \mathrm{N}$ and $6^{\circ} \mathrm{W}-18^{\circ} \mathrm{W}$ (Figure 1). The basin is characterized by irregular rainfall patterns, with a clear decreasing gradient from South to North, corresponding to Guinean, Soudanian and Sahelian climate zones. The Sahelian rainfall pattern presents a single rainy season that lasts seven months in the South and three months in the North (LEBEL at al., 2003).

Simulated rainfall in the REMO RCM (JACOB, 2001; PAETH et al., 2005), with a horizontal resolution of $0.5^{\circ}$, was used in this study. The REMO model is a hydrostatic regional climate model that has been developed at the Max-Planck Institute for Meteorology (JACOB, 2001; PAETH et al., 2005). The REMO dataset used correspond to databases provided by AMMA (African Monsoon Multidisciplinary Analyses) European Project.

The simulated precipitation was compared to the gridded observations by CRU (NEW et al., 2000) and IRD (Institut de Recherche pour le Développement) monthly rainfall data sets. IRD dataset corresponds to on-site daily rainfall data, interpolated for a regular grid of $1^{\circ}$ (DIEDHIOU et al., 2001). The IRD data set is widely used and provide valuable rainfall measurements (DIEDHIOU et al., 1999; JANICOT and SUlTAN, 2001; MESSAGER et al., 2004)

The CRU data set corresponds to a long-term climatology that covers Africa with a regular $0.5^{\circ}$ grid. A number of authors (POCCARD et al., 2000) have shown that CRU dataset provides a good sample of spatial-temporal rainfall distribution in West Africa. Figure 1 shows the Senegal River basin location and the spatial pattern of mean annual rainfall in the $1961-1990$ period, according to CRU data. Some of the locations were selected within Senegal Basin (Figure 1), for the evaluation of the REMO model and the impact analysis in dry spells.

\section{Analysis of annual rainfall and seasonal cycle}

The spatial rainfall pattern and its seasonal cycle were analysed from mean monthly rainfall, following three 

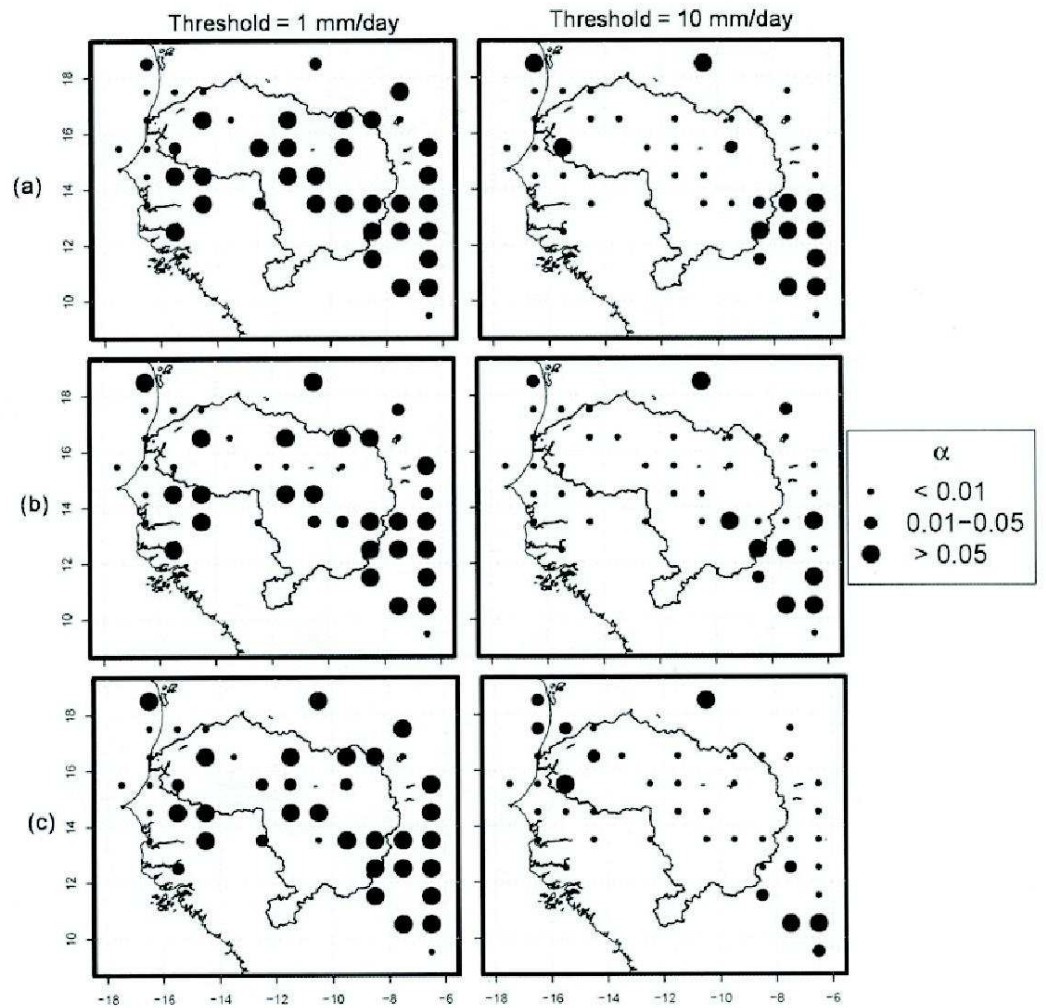

Figure 3: Spatial distribution of results of DSL bias analysis between 1970-1990, where $\alpha$ is the Kolmogorov-Smirnov goodness of fit test p-value: (a) First, (b) second and (c) third REMO ensemble member.

Table 3: Results of Mann-Kendall Test. Tau statistic and significance, for an average over the three REMO ensemble members.

\begin{tabular}{|c|c|c|c|c|c|}
\hline \multicolumn{3}{|c|}{ Upper basin } & \multicolumn{3}{|c|}{ Medium and lower basin } \\
\hline \multicolumn{6}{|c|}{ Moderate daily rain events } \\
\hline Period & Tau & 2-sided p-value & Period & Tau & 2-sided pvalue \\
\hline $1961-1990$ & 0.163 & 0.2117 & $1961-1990$ & 0.0897 & 0.4978 \\
\hline $1991-2020$ & -0.0713 & 0.5925 & $1991-2020$ & -0.103 & 0.4325 \\
\hline $2021-2050$ & -0.0667 & 0.6174 & $2021-2050$ & -0.228 & 0.0804 \\
\hline \multicolumn{6}{|c|}{$\begin{array}{l}\text { Heavy daily rain } \\
\text { events }\end{array}$} \\
\hline Period & Tau & 2-sided p-value & Period & Tau & 2-sided pvalue \\
\hline $1961-1990$ & 0.237 & 0.0688 & $1961-1990$ & 0.0184 & 0.9006 \\
\hline $1991-2020$ & -0.0299 & 0.8305 & $1991-2020$ & 0.147 & 0.2609 \\
\hline $2021-2050$ & 0.0207 & 0.8865 & $2021-2050$ & -0.172 & 0.1868 \\
\hline \multicolumn{6}{|c|}{$\begin{array}{l}\text { Very heavy daily rain } \\
\text { events }\end{array}$} \\
\hline Period & Tau & 2-sided p-value & Period & Tau & 2-sided pvalue \\
\hline $1961-1990$ & 0.341 & 0.0087 & 1961-1990 & 0.0644 & 0.6300 \\
\hline $1991-2020$ & 0.0161 & 0.9146 & $1991-2020$ & 0.251 & 0.0540 \\
\hline $2021-2050$ & 0.18 & 0.1695 & $2021-2050$ & 0.053 & 0.6946 \\
\hline
\end{tabular}



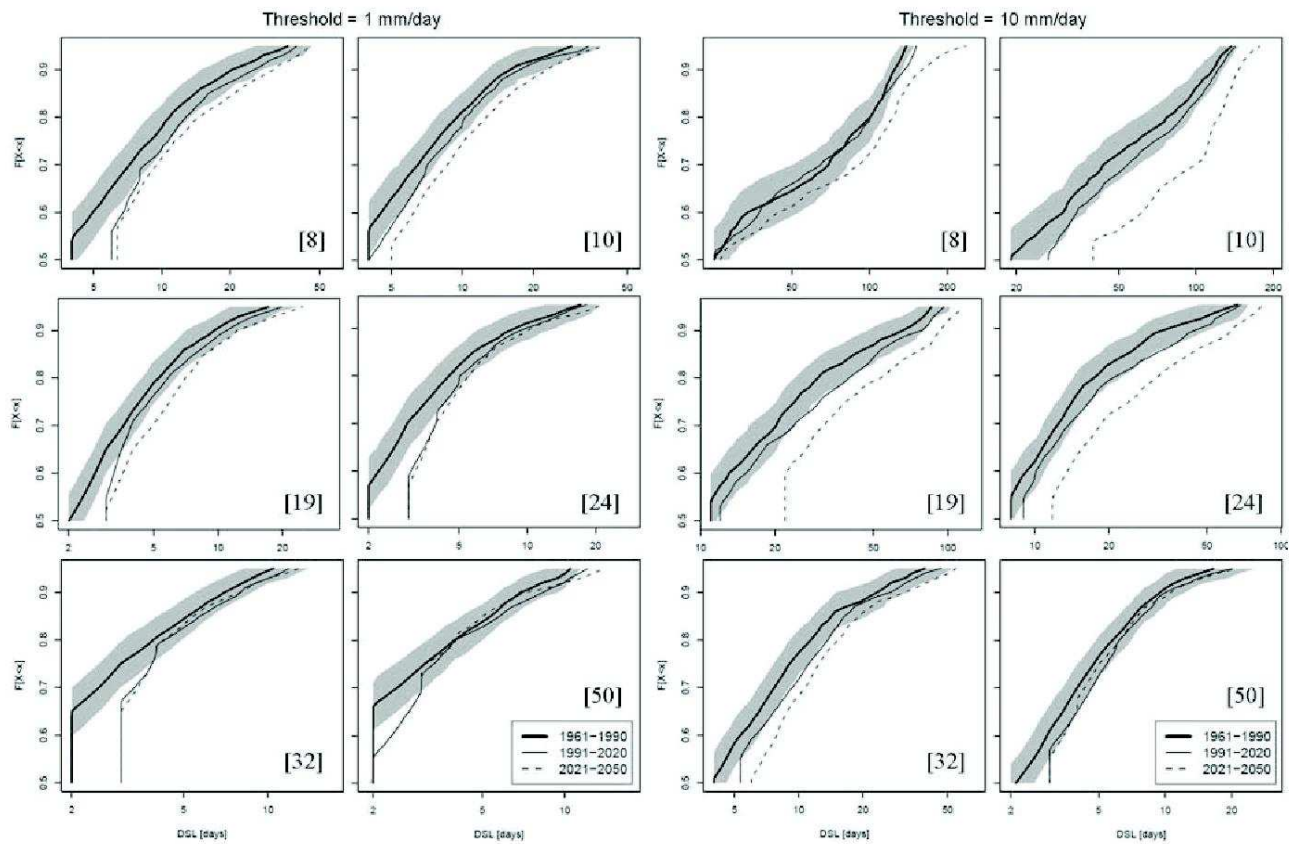

Figure 4: Empirical cdf of DSL time series as an average over the three REMO ensemble members. The gray polygon is the cdf $95 \%$ confidence interval (CI) in 1961-1990 period.

REMO model ensemble members: first (REMO-901 and REMO-911), second (REMO-902 and REMO-912), and third (REMO-903 and REMO-913). The simplest analysis method for systematic spatial errors consists of representing the difference between the mean annual simulated rainfall (RMODEL) and observed rainfall (RCRU) grids, as in previous studies using the REMO model, e.g. by PAETH et al. (2005) using ERA/ECMWF analysis driven runs, and by PAETH et al. (2009) analyzing ECHAM5 driven runs on 20th century. REMO and CRU data can be adjusted by performing a hypothesis test to obtain the multi-annual rainfall average. From the value of significance value $(\alpha)$ of hypothesis test of the mean annual rainfall, considering 1961-1990 period, spatially represented for REMO-901, REMO-902 and REMO903 data (not shown), REMO datasets show a reliable multi-annual rainfall average in the Senegal basin. Nev ertheless, the actual rainfall amount was underestimated, especially in the Sahelian region. PAETH et al. (2005) explained the model behavior in the representation of spatial rainfall pattern.

Some of the cells were selected for the Senegal River basin (points $8,10,16,19,24$ and 32), taking into account the CRU and IRD data availability. Table 1 shows the estimated RMSE/RCRU ratios for these points, con- sidering the mean annual rainfall from CRU (RCRU) data. Figure 2 refers to the simulated and observed mean seasonal cycle, taking into account an average over the three ensemble REMO members (REMOAVER) and also CRU and IRD data sets. Except for point 32 which represents the Soudanian region, all of the REMO implementations underestimate the rainfall values for the studied points.

\section{Analysis of Dry Spell Length (DSL)}

\section{Bias of monsoon DSL}

The monsoon season has been considered as 1 May-30 September. The dry spell length (DSL) was defined as the number of consecutive days with rainfall amounts below a threshold ( $1 \mathrm{~mm} /$ day or $10 \mathrm{~mm} /$ day $)$ for the monsoon season. The selected dry spells belong to the monsoon season, with special cases of spells that were not totally confined into that period. Even the larger spells could belong to several years, but according to definition, only the spell days in the monsoon seasons added up to length of dry spells.

According to the previous definition, the time series of monsoon DSL were obtained for both REMO and IRD 

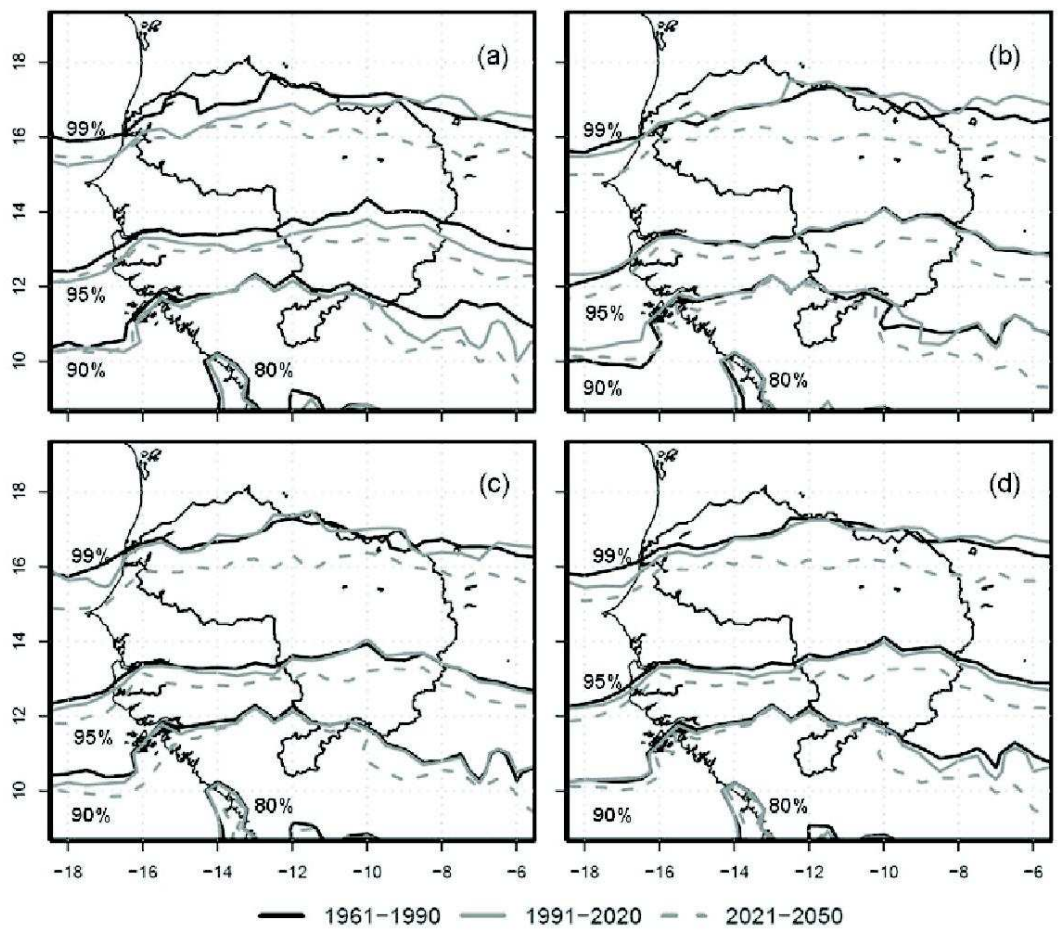

Figure 5: Percentage of days with rainfall below $10 \mathrm{~mm} /$ day: (a) First, (b) Second and (c) Third REMO ensemble member; (d) Average over the three REMO ensemble members.

data. Because the IRD daily precipitation grids are not continuous, the DSL bias analysis was carried out on locations with time series of rainfall between 01/01/1970 and $31 / 12 / 1990$. In the selected locations, the p-value of two-sample Kolmogorov-Smirnov goodness of fit test was computed between the DSL time series from IRD and each REMO ensemble member (SHESKIN, 2000; MARQUES DE SA, 2003). The results of bias analysis (Figure 3), demonstrate that $1 \mathrm{~mm} /$ day threshold DSLs are well fitted by REMO runs (most of the locations with $\alpha>0.05$ ), while the $10 \mathrm{~mm} /$ day threshold DSLs show worse p-values (most of the locations with $\alpha<0.01$ ). Anyway, the test shows that the DSLs are best represented by REMO runs in Soudanian zone for both considered thresholds.

\section{Projected changes in DSL high quantiles from REMO ensemble members}

DSL time series of 30 years were derived from REMO ensemble members (for 1961-1990, 1991-2020 and 2021-2050 time periods). In selected locations from
DSL time series, empirical cumulative distribution functions (cdf) using bootstrap re-sampling, were estimated (EFRON and TIBSHIRANI, 1993; FOX, 2002). From the results presented in Figure 4 and Table 2, the empirical cdf for 1991-2020 and 2021-2050 time periods, are higher than 1961-1990 cdf. However, not all the cases are significant. The DSL differences for $1 \mathrm{~mm} /$ day threshold, are significant in locations 8,10 and 19 only over 2021-2050 time period. On the other hand, 10 mm/day threshold (Figure 4 and Table 2), presents significant differences in the whole of locations for 20212050 time period, but the differences are not significant for 1991-2020 time period.

It is remarkable that the dry spell events spread through the hydrological processes of the water cycle, and have an influence on wetlands seasonal cycle. Therefore, they reduce aquifer recharge and subsequently have a negative impact on the region's agriculture activities. 

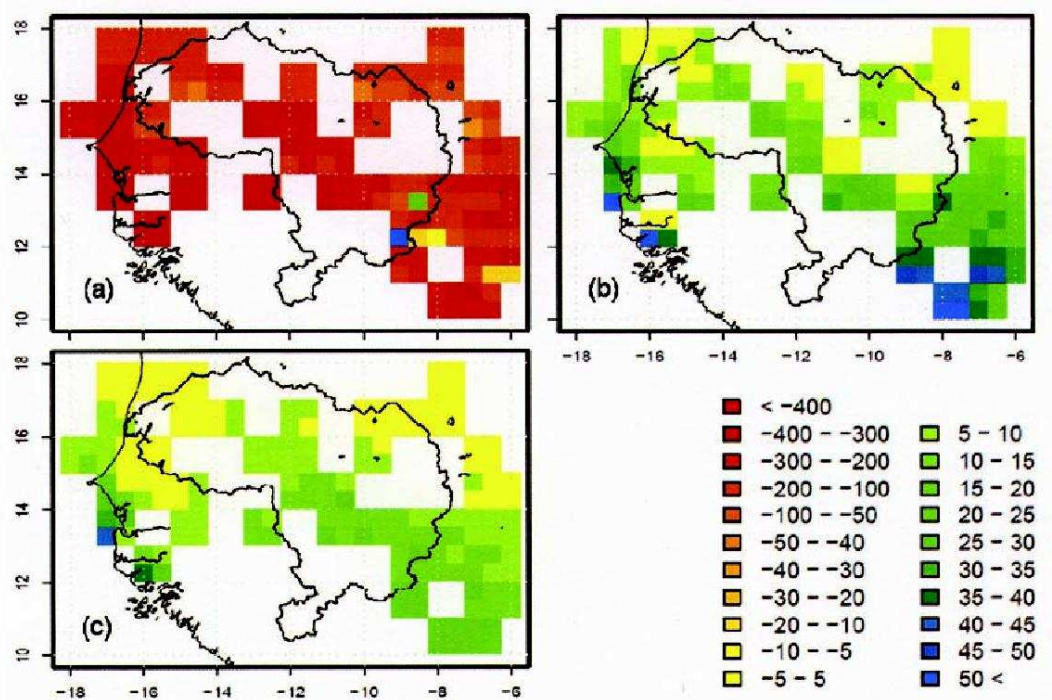

Figure 6: Absolute differences in number of daily rainy events between REMO (average of the three ensemble members) and IRD data for 1970-1990 period: (a) Moderate, (b) Heavy, and (c) Very heavy events. Positive values represent overestimation.

\section{Analysis of daily rainy events}

The percentage of rainfall days with less than 10 $\mathrm{mm} /$ day for three time periods (1961-1990, 1991-2020 and 2021-2050), for the three REMO ensemble members, is presented in Figure 5. For the 1991-2020 time period, there is a displacement of the isolines to the North and a higher frequency of rainy days. Nonetheless, there is a displacement of the isolines to the South and a lower frequency of rainy days for 2021-2050 time period. Therefore, an analysis of the trends of daily rainfall events should be encouraged.

Due to the South-North decreasing gradient of rainfall, we divided the basin in two areas for the analy sis: upper basin $\left(10^{\circ} \mathrm{N}-14^{\circ} \mathrm{N}\right)$, and medium and lower basin $\left(14^{\circ} \mathrm{N}-18^{\circ} \mathrm{N}\right)$. Different types of daily rainfall events were considered: moderate events $(5 \leq \mathrm{R}<50$ $\mathrm{mm} /$ day), heavy events ( $50<\mathrm{R}<100 \mathrm{~mm} /$ day), and very heavy events ( $R \geq 100 \mathrm{~mm} /$ day). The bias of the average over the three REMO ensemble members with respect to IRD dataset is presented in Figure 6 . An overestimation for the number of very heavy rainy events is found for all locations. Underestimations in the number of moderate events are evident

The spatial distribution in these types of events for the 1961-1990 and the 2021-2050 period, are shown in Figure 7 for an average over the three REMO ensemble members. The accumulated time series of daily rainfalls events by year for each basin area, considering the aver- age of three REMO ensembles members, are presented in Figure 8 as box plots. A clear decrease in the number of rainy events for 2021-2050 is observed in all cases, in comparison with 1961-1990 time period. This decrement is clear for moderate and heavy rainy events.

Mann-Kendall non-parametric test has been applied (KENDALL, 1975) in order to estimate the significance of temporal trends of annual events quantity for each of the areas in which the basin was divided (Table 3 ). This Test has been widely employed for the analysis of hydrological series (KUNDZEWICZ and ROBSON, 2000; YUE et al., 2002; KHALIQ et al., 2009).

The upper basin exhibit significant increment of very heavy rainy events for 1961-1990. The trend is not that significant in the other areas, taking into account moderate and heavy daily rainy events.

\section{Conclusions}

Our research shows that Sahelian and Soudanian regions exhibit different patterns of climate fluctuations. LEBEL et al. (2009), among others, have presented similar conclusions and they explain this fact to the West African Monsoon (WAM) control on West Africa climate and the effect of possible changing surface conditions in the rainfall variability pattern.

The present study agrees with the analysis by MODARRES (2009) in that the spatial distribution of statistical characteristics of extreme dry events, is a key 

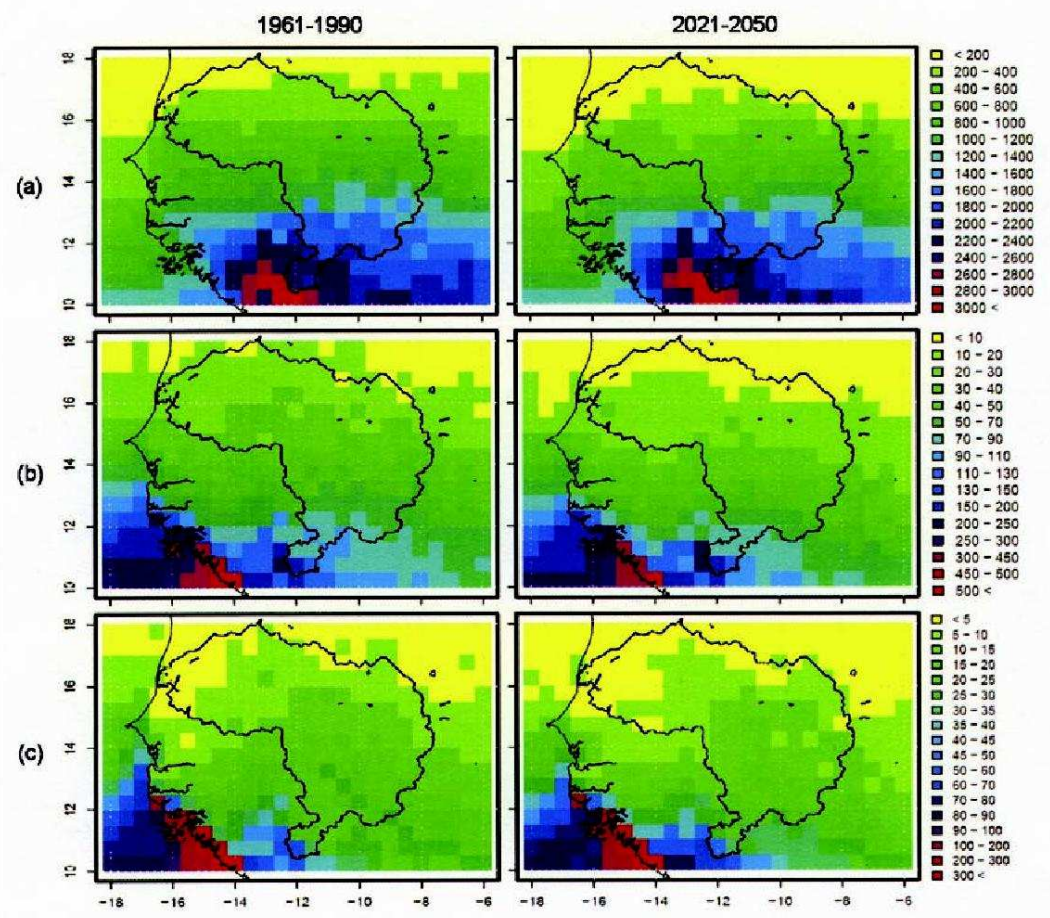

Figure 7: Number of daily rainy events: (a) Moderate, (b) Heavy, and (c) Very heavy events, for an average over the three REMO ensemble members.

practice for further management. The analyses made show an increase in the dry spells length computed via empirical cdf, being greater in the Sahelian region. Also, the analyses assessed greatest DSL differences over 2021-2050 time period.

From the results obtained in the evaluation of the capabilities of the three REMO ensemble members for modeling monsoon rainfall, we conclude REMO runs are suitable in order to estimate empirical quantiles of rainfall threshold ( $1 \mathrm{~mm} /$ day and $10 \mathrm{~mm} /$ day) for the whole basin. However, the best performance was observed for the Soudanian zone.

According to REMO projection, an increment on empirical quantiles of $1 \mathrm{~mm} /$ day threshold DSL is expected, especially in the Sahelian zone for 2021-2050 time period. On the other hand, for 1991-2020 time period the increment of DSL higher quantiles was not significant. The empirical quantiles for $10 \mathrm{~mm} /$ day threshold DSL demonstrated a significant rise for the whole basin. Nevertheless, in the same way as $1 \mathrm{~mm} / \mathrm{day}$ threshold, the increases are not meaningful for 19912020 time period.
In general, an overestimation of the number of very heavy events and underestimation of moderate events are found for 1970-1990 time period considering the IRD dataset. The study areas exhibit upward trends in the frequency of very heavy daily rainy events and downward trends of moderate rainy events, for 20212050 , but not significant.

Climate models generally do not provide a right rainfall representation (ERRICO et al., 2001; LIU and MONCRIEFF, 2001), due to physical parameterizations related to cloud formations and rainfalls. More research is needed in this field. The present work should continue in order to include the new West Africa RCMs published by ENSEMBLE project (CHRISTENSEN et al., 2009), and to create risk maps containing extreme weather events - from dry spells to rainstorms -, according to RCM data and other studies and applying regional frequency analysis techniques.

\section{Acknowledgments}

This work was performed within the framework of the AMMA project. Based on a French initiative, AMMA 
Upper basin

(a)

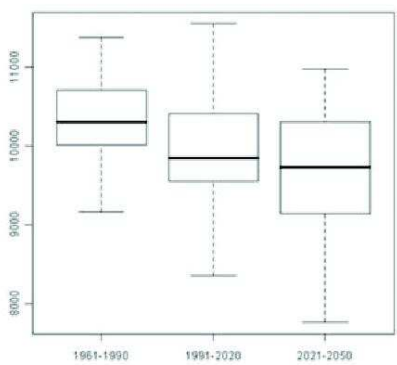

(b)

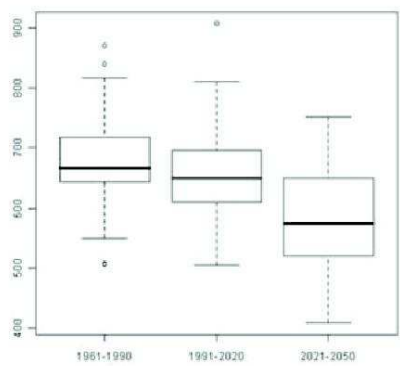

(c)

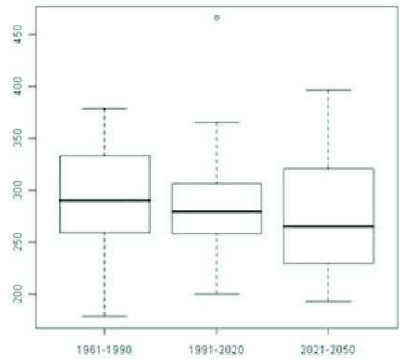

Lower and medium basin
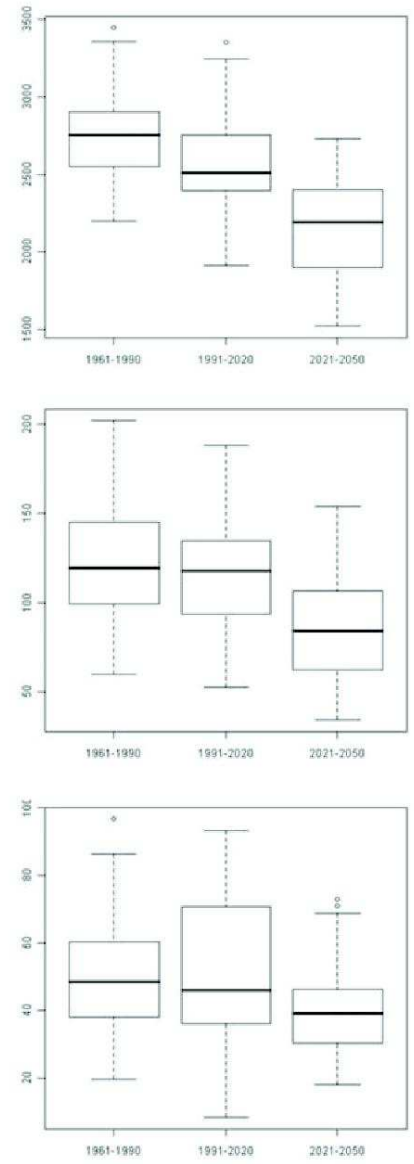

Figure 8: Box plots of cumulative number of: (a) moderate, (b) heavy rainy events, and (c) very heavy rainy events, at annual scale, for an average over the three REMO ensemble members

has been constructed by an international group and is currently funded by large number of agencies, especially from France, the UK, the US, and Africa. It has been the beneficiary of a major financial contribution from the European Community's Sixth Framework Research Programme. Detailed information on the scientific coordination and funding is available on the AMMA international web site (https:/www.amma-eu.org). Special thanks to PhD Heiko Paeth (Germany), and his team, for his contribution to AMMA Community, who has made possible the access to RCM and REMO studies to achieve the aims of impact studies in water resources. We acknowledge to IRD and PhD Jan POLCHER, for providing observed daily rainfall. We appreciate the R\&D Project CGL2008-02530/BTE of Spanish Ministry of Science and Innovation support

\section{References}

CHRISTENSEN, J.H., M. RUMMUKAINEN, G. LENDERINK, 2009: Formulation of very-high-resolution regional climate model ensembles for Europe. - In: VAN DER LINDEN, P. 
J.F.B. MiTCHELL (Eds): ENSEMBLES: Climate change and its impacts: Summary of research and results from the ENSEMBLES project. Met Office Hadley Centre, FitzRoy Road, Exeter EX1 3PB, UK, 160 pp.

Descroix, L., G. Mahé, T. Lebel, G. FavreaU, S. GALle, E. GAUTIER, J.C. Olivry, J. AlbergeL, O. Amogu, B. Cappelaere, R. Dessouassi, A. DiedHIOU, E. LE BRETON, I. MAMADOU, D. SIGHOMNOU, 2009: Spatio-temporal variability of hydrological regimes around the boundaries between Sahelian and Sudanian areas of West Africa: A synthesis. - J. Hydrol. 375, 90-102.

DIEDHIOU, A., S. JANICOT, A. VILTARD, P. DE FELICE, H. LAURENT, 1999: Easterly wave regimes and associated convection over West Africa and tropical Atlantic: result from the NCEP/NCAR and ECMWF reanalyses. - Climate Dynam. 15, 795-822.

Diedhiou, A., S. JANicot, A. ViltaRd, P. DE FÉLICE, 2001: Composite patterns of easterly wave disturbances over West Africa and the tropical Atlantic: A climatology from the 1979-95 NCEP/NCAR reanalyses. - Climate Dy nam. 18, 241-253.

EC, 2009. Common Implementation Strategy for the Water Framework Directive (2000/60/EC). - TechniWater Framework Directive (2000/60/EC). - Techni-
cal Report-2009-040, European Comunities, 52 pp cal Report-2009-040, European Comunities, $52 \mathrm{pp}$.,

EFRON, B., R.J. THISHIRANI, 1993: An introduction to the bootstrap. - Monographs on Statistics \& Applied Probability 57. New York, Chapman and Hall, $436 \mathrm{pp}$.

ERRICO, R.M., D.J. STENSRUd, K.D. RAEDER, 2001: Estimation of the error distributions of precipitation produced by convective parameterization schemes. - Quart. J. Roy. Meteor. Soc. 127, 2495-2512.

FoX, J., 2002: Bootstrapping regression models. - Appendix to An R and S-PLUS Companion to Applied Regression, 14 pp., http://cran.r-project.org/doc/contrib/FoxCompanion/

Grist, J.P., S.E. Nicholson, 2001: A study of the dynamic factors influencing the interannual variability of rainfall in the West African Sahel. - J. Climate 14, 1337-1359.

JACOB, D., 2001: A note to the simulation of the annual and interannual variability of the water budget over the Baltic Sea drainage basin. - Meteor. Atmos. Phys. 77, 61-74.

JANICOT, S., B. SULTAN, 2001: Intra-seasonal modulation of convection in the West African monsoon. - Geophys. Res. Lett. 28, 523-526.

KendaLL, M.G., 1975: Rank Correlation Methods. Charles Griffin, London.

KHALIQ, M.N., T.B.M.J. OUARDA, P. GACHON, 2009 Identification of temporal trends in annual and seasonal low flows occurring in Canadian rivers: The effect of short- and long-term persistence. - J. Hydrol. 369, 183-197.
KundZEwICZ, Z.W., A. RoBson, 2000: Detecting trend and other changes in hydrological data. - World Climate Program - Water. - WMO/UNESCO, WCDMP-45, WMO/TD 1013, Geneva, 157 pp.

LEBEL, T., A. ALI, 2009: Recent trends in the Central and Western Sahel rainfall regime $(1990-2007)$. - J. Hydrol. $375,52-64$.

Lebel, T., A. Diedhiou, H. LAurent, 2003: Seasonal cycle and interannual variability of the Sahelian rainfall at hydrological scales. - J. Geophys. Res. 108, 1401-1411.

Lebel, T., B. Cappelaere, S. Galle, N. Hanan, L. Kergoat, S. LeVis, B. Vieux, L. Descroix, M. Gosset, E. MOUGIN, C. PEUGEOT, L. SEguis, 2009 AMMA-CATCH studies in the Sahelian region of WestAfrica: An overview. - J. Hydrol. 375, 3-13.

LIU, C., M.W. MONCRIEFF, 2001: Cumulus ensembles in shear: Implications for parameterization. - J. Atmos. Sci 58, 2832-2843.

MARQUES DE SA, J.P., 2003: Applied statistics using SPSS, STATISTICA, and MATLAB. - Springer-Verlag, Berlin, Germany, 505 pp.

Messager, C., H. Gallée, O. BRAsseur, 2004: Precipitation sensitivity to regional SST in a regional climate simulation during the West African monsoon for two dry years. Climate Dynam. 22, 249-266.

MODARRES, R., 2009: Regional dry spells frequency analysis by L-Moment and Multivariate analysis. - Water Resour. Manag., published online, DOI:10.1007/s11269-0099556-5.

New, M., M. Hulme, P. Jones, 2000: Representing twentieth-century space-time climate variability. Part II: Development of 1901-96 monthly grids of terrestrial surface climate. - J. Climate 13, 2217-2238.

PAETH, H., K. BORN, R. PODZUN, D. JACOB, 2005: Regional dynamical downscaling over West Africa: mode evaluation and comparison of wet and dry years. - Meteorol. Z. 14, 349-367.

PAETH, H., K. BORN, R. GIRMES, R. PODZUN, D. JACOB 2009: Regional climate change in tropical and Northem Africa due to greenhouse forcing and land use changes. J. Climate 22, 114-132.

POCCARD, I., S. JANICOT, P. CAMBERLIN, 2000: Comparison of rainfall structures between NCEP/NCAR reanalyses and observed data over tropical Africa. - Climate Dynam. 16, 897-915.

SHESKIN, D.J., 2000: Handbook of Parametric and Nonparametric Statistical Procedures, 2nd ed. - Chapman \& Hall/CRC, Boca Raton, FL, 1016 pp.

Yue, S., P. PILON, G. CAVAdIAS, 2002: Power of the MannKendall and Spearman's rho test for detecting monotonic trends in hydrological series. - J. Hydrol. 259, 254-271. 


\subsection{Resumen del Artículo 2}

\subsubsection{Título}

Assessing the impact of climate variability and climate change on runoff in West Africa: the case of Senegal and Nakambé River basins.

\subsubsection{Objetivos específicos}

- Mejorar las predicciones de los impactos del cambio climático en los eventos meteorológicos extremos en la Cuenca del Río Senegal (África Occidental).

- Obtener los patrones espacio-temporales de la precipitación en la zona de estudio, analizando el comportamiento interanual y las tendencias de las propiedades estadísticas de la lluvia.

- Integrar la información de precipitación diaria, a partir de campos o mallas derivados desde Modelos Climáticos Regionales (RCM) y Globales, en un ambiente de Sistemas de Información Geográfica (SIG).

\subsubsection{Metodología}

Las regiones de estudio, en este trabajo, han correspondido a las Cuencas de los Ríos Senegal y Nakambé (África Occidental). La Cuenca del Río Senegal se caracteriza por la irregularidad espacial y temporal de la precipitación, que tiene un claro gradiente latitudinal, con precipitaciones mayores a 2000 mm/año en las montañas Fouta Djalon al Sur, hasta menores a $200 \mathrm{~mm} / \mathrm{año}$ en la parte baja de la cuenca al Norte. La Cuenca del Río Nakambé está ubicada en la parte más alta de la Cuenca del Río Volta, en Burkina Faso, y presenta una precipitación media de 700 mm/año. Ambas cuencas fueron divididas de acuerdo con la definición de ecorregiones propuesta por Olson et al. (2001), y se escogieron sitios de análisis dentro de cada ecorregión, cumpliendo con las restricciones en la distribución espacial impuestas por los datos provistos por el IRD.

En este trabajo, se utilizaron mallas de datos observados interpolados suministrados por el CRU y provistos por el IRD. Adicionalmente, en la Cuenca del Río Nakambé se han utilizado datos de precipitación, caudales desde estaciones de medición y datos de evapotranspiración potencial estimada según el método de Makkink (Xu and Singh, 2002), a partir de los datos climáticos proporcionados por los RCMs. Por último, datos de simulaciones de RCMs (KNMI/RACMO, MPI/REMO y SMHI/RCA), pertenecientes a los proyectos ENSEMBLES e IMPETUS, fueron utilizadas para realizar el análisis de tendencias. 
Se compararon los ciclos estacionales calculados, con los datos observados y simulados desde RCMs en el periodo 1970-1990. Los ciclos estacionales fueron calculados como un promedio para todos los sitios de análisis dentro cada una de las ecorregiones.

Se construyeron las series de longitud de rachas secas (DSL) en la estación monzónica (número consecutivo de días con precipitación menor que $1 \mathrm{~mm} /$ día, entre el 1 de mayo y 30 de septiembre). Las series temporales de rachas secas se agregaron para cada ecorregión. Los estadísticos básicos de las DSL (media y desviación estándar) fueron estimados desde los datos observados IRD y simulados por los RCMs.

Para analizar la tendencia de las PDF de la precipitación anual y de las series de longitud de racha seca máxima anual (AMDSL), se utilizó la metodología GAMLSS, considerando cuatro distribuciones de probabilidad de dos parámetros (Gumbel, Gamma, Lognormal y Weibull), ampliamente utilizadas en hidrología.

El modelo GR2M de simulación continua a escala mensual (Maklouf, 1994), fue utilizado para la simulación hidrológica en la Cuenca del Río Nakambé. El modelo hidrológico fue calibrado (utilizando el periodo 1980-1989) y validado (con el periodo 1990-1999) calculando el coeficiente de Nash (Nash and Sutcliffe, 1970), utilizando como referencia los datos observados del IRD y de las estaciones sinópticas. Una vez calibrado y validado, el modelo se forzó con los datos simulados por los tres RCMs anteriormente mencionados. Como no se consideraron escenarios de cambio de uso del suelo, los parámetros del modelo permanecieron constantes durante la simulación. Para la simulación hidrológica, el sesgo de los datos de precipitación y evapotranspiración potencial obtenidos desde los RCM, fue corregido utilizando los promedios mensuales y anuales de los datos observados en el periodo 1961-1990.

\subsubsection{Resultados}

Como se esperaba, el ciclo estacional representado por ambas bases de datos observadas ( $C R U$ e IRD) presenta una alta convergencia, aumentando la confianza sobre su consistencia. Los RCMs presentan diferente habilidad para simular el ciclo anual de la precipitación, siendo el RCM KNMI/RACMO el que mejor se comporta.

Desde los datos observados, la longitud media de las DSL en el Sahel duplica el valor para la zona Sudaniana. Además, la variabilidad de las DSL, representada por la desviación estándar, también es mayor en la ecorregión del Sahel.

El valor medio de las DSL es mejor simulado también por el RCM KNMI/RACMO. La desviación estándar de las DSL es mejor simulada por el MPI/REMO en la zona del Sahel, mientras que el KNMI/RACMO es más hábil en la zona Sudaniana. El RCM 
SMHI/RCA presenta el menor nivel de desempeño para simular las DSL en todas las regiones consideradas.

De acuerdo con el análisis de tendencias realizado con GAMLSS, el RCM KMNI/RACMO predice un decrecimiento de la cantidad de precipitación en la Cuenca del Río Senegal, mientras que predice un aumento para la Cuenca del Nakambé. El RCM MPI/REMO predice un descenso cercano al 30\% en la cantidad de precipitación para todas las regiones analizadas. Finalmente, el RCM SMHI/RCA muestra una fuerte tendencia creciente de la precipitación en la Cuenca del Río Senegal, mientras que predice un descenso en la Cuenca del Río Nakambé. Como se observa, los RCMs no convergen al predecir la tendencia futura de la precipitación.

También se aplicó el análisis GAMLSS a las series AMDSL registradas en cada ecorregión. Los modelos estadísticos ajustados revelan un incremento general de las AMDSL (Figura 8, mencionada pero no visualizada en el artículo). Los RCM KNMI/RACMO y MPI/REMO proyectan un incremento del percentil 95\% de las AMDSL entre el 3-25\% (3-35 días) para la zona Saheliana de la Cuenca del Senegal, mientras que para la zona Sudaniana el incremento proyectado corresponde a 12-36\% (6-12 días). Para la Cuenca del Río Nakambé, KNMI/RACMO y MPI/REMO proyectan un aumento de las AMDSL-95\% (16-47\%, 3-18 días). Mientras el RCM SMHI/RCA predice descensos de las AMDSL en toda la Cuenca del Río Senegal.

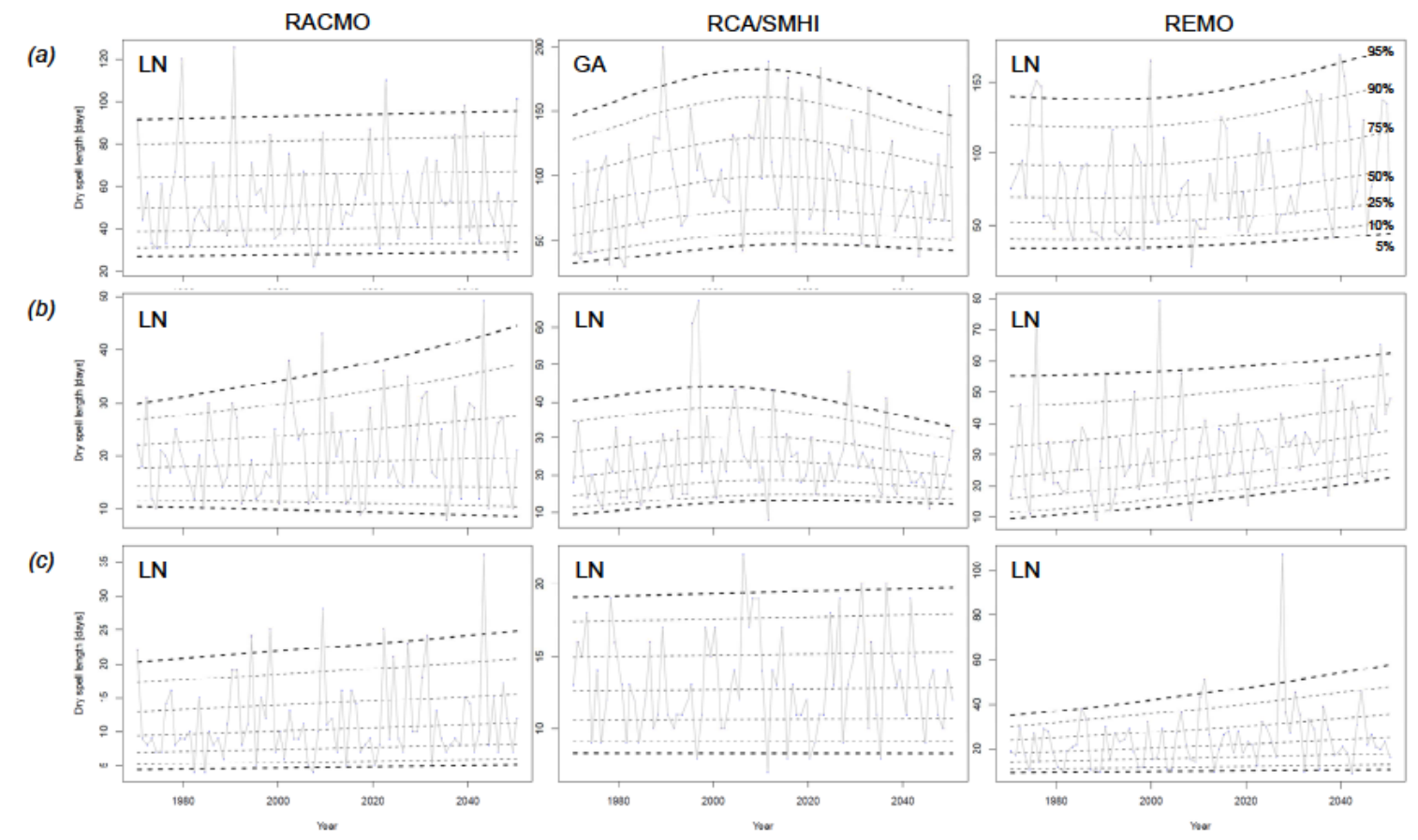

Figura 8. Análisis GAMLSS de las AMDSL. (a) SAS en la Cuenca del Senegal, (b) WSS en la Cuenca del Senegal y (c) Cuenca del Nakambé. Las curvas del los centiles (5-95\%) están representadas por líneas discontinuas. 
En cuanto a la simulación hidrológica, se alcanzó un buen ajuste del modelo hidrológico GR2M. Se lograron valores del coeficiente de Nash superiores al 70\%, tanto en el proceso de calibración como de validación. Al igual que en las tendencias de la precipitación, las tendencias plausibles de los caudales en el Río Nakambé difieren de un RCM a otro. Sin embargo, las tendencias de los caudales no están en concordancia con el forzamiento impuesto por otras variables climáticas. Aunque los RCMs SMHI/RCA y MPI/REMO predicen un descenso de la cantidad de precipitación, los caudales simulados con estos modelos presentan tendencias crecientes.

El resultado anterior pone de relieve la dificultad de predecir la respuesta de los sistemas en esta región semiárida. Cómo lo han demostrado las observaciones (Mahé et al., 2005), un descenso de la precipitación no implica un descenso de los caudales. Se debe considerar la influencia de los cambios en el uso del suelo en el ciclo hidrológico. Como apunta Mahé et al. (2005), el incremento de las superficies impermeables y las áreas cultivadas, en detrimento de las coberturas vegetales naturales, ha llevado a una pérdida de capacidad de infiltración de la superficie y un aumento de la escorrentía. Se deben desarrollar entonces escenarios de cambio en el uso del suelo para integrarlos en los modelos hidrológicos, en conjunto con los escenarios plausibles de cambio climático.

\subsubsection{Conclusiones y recomendaciones}

Desde los datos provistos por los RCMs, se analizaron las tendencias en la precipitación anual y en la longitud de las rachas secas. El comportamiento no estacionario de las series de AMDSL en la temporada monzónica, se refleja en los cambios de la media y la varianza.

Los análisis del desvío demostraron que el RCM KNMI/RACMO presenta mejores habilidades para simular el ciclo anual de la precipitación, especialmente en la zona Sudaniana. Adicionalmente, este RCM simula con gran confiabilidad la distribución de las DSL. Por otro lado, el RCM SMHI/RCA no presenta una buena simulación de las variables analizadas (precipitación y DSL).

Para el horizonte 2050, se proyecta un decrecimiento de la precipitación en todas las ecorregiones, de acuerdo con los RCM KNMI/RACMO y MPI/REMO, excepto en la Cuenca del Nakambé, donde MPI/REMO predice un aumento de la precipitación. Por otra parte, las AMDSL presentan una tendencia creciente de acuerdo con estos RCM, especialmente en la región Sudaniana de ambas cuencas.

A pesar de la tendencia decreciente de la precipitación, el análisis de impacto con el modelo hidrológico en la Cuenca del río Nakambé mostró una tendencia creciente de los caudales. Esta tendencia revela la importancia de considerar explícitamente los 
usos del suelo (o cobertura vegetal), en los procesos del ciclo hidrológico que determinan la disponibilidad de los recursos hídricos en el Sahel. Los análisis deben extenderse a otras cuencas de la región, y datos desde otros RCM deben ser utilizados. Finalmente, el desarrollo de escenarios de cambio en el uso del suelo es necesario para desarrollar herramientas que apoyen efectivamente las decisiones que tienen como objeto una mejor adaptación al cambio climático en el Sahel. 



\title{
5.2.6 Copia de la publicación
}

ATMOSPHERIC SCIENCE LETTERS

Atmos. Sci. Let. 12: 109-I I5 (201 I)

Published online in Wiley Online Library

(wileyonlinelibrary.com) DOI: 10.1002/as1.317

\section{Assessing the impact of climate variability and climate change on runoff in West Africa: the case of Senegal and Nakambe River basins}

\author{
H. Karambiri, I* S. G. García Galiano, ${ }^{2}$ J. D. Giraldo, ${ }_{1}^{2}$. Yacouba, 'B. Ibrahim, ' B. Barbier' and J. Polcher ${ }^{3}$ \\ I intemational Institute for Water and Environmental Engineening (2iE), Ouagadougou, Bunkina Faso \\ ${ }^{2}$ Department of Civil Engineering, Technical University of Cantagena, Cartagena, Spain \\ 3 Laboratoire de Météorologie Dynamique du CNRS/IPSL, Université Pierre et Marie CURIE, Paris, France
}

*Correspondence to:

H. Korambin, Intemationo

institute for Water and

Environmental Engineering (2iE)

OI BP 594 Ouggadougou O

Burkino Faso.

E-mail:

harounakarambin@2ie-edu.org

Received: I March 2010

Revised: 16 November 2010

Accepted: 17 Novernber 201

\begin{abstract}
West Africa and its people are very vulnerable to climate variability and changes. Increasing the knowledge of plausible trends of rainfall dry spell lengths (DSL) in the rainy season, and of runoff, enables the assessment of vulnerability and adaptive capacity of the system. These predictions are crucial from a water management and policy perspective. The analyses based on regional climate models (RCMs) and observed datasets exhibit non-stationary behavior and an increase of DSL. Our results highlight the difficulty of selected RCMs to reproduce present climate and their divergence in predicting future climate. Impacts on water resources depend not only on climate forcing but also on land surface conditions. Copyright $\odot 2011$ Roval Meteorological Society
\end{abstract}

Keywords: climate change and variability; regional climate model; impact studies; droughts; runoff; West Africa

\section{Introduction}

Due to the close link between climate and socioeconomic activities, societies in West African are more vulnerable to rainfall variability and changes. The region has experienced a number of severe droughts since the end of the 1960 s. Due to rapid population growth, the pressures on the environment and natural resources have been exacerbated, leading to profound changes in land uses. The impacts of historical climate variability and changes, combined with the effects of land use changes on hydrological processes and water resources, are now well documented (Descroix et al., 2009; Favreau et al., 2009; Séguis et al., 2011). There is evidence of contrasted responses by hydrological and hydrogeological systems, especially in the West African Sahel (Leduc et al., 2001; Mahé et al, 2005; Mahé and Paturel, 2009). However, there have been few attempts to deal with future climate variability and changes, and their impacts on runoff. This issue was one of the main concerns of the African Monsoon Multidisciplinary Analyses (AMMA) project (Polcher et al., 2011)

This article focuses on the analysis of the historic and regional climate model (RCM) predicted rainfall regime for the Senegal and Nakambe River basins and its impact on the monsoon dry spell lengths (DSL) on both basins. Impacts on river flows are also predicted for the Nakambe basin.

\section{Materials and methods}

\section{I. Study area}

The Senegal and Nakambe River basins, which are both strongly affected by climate and land use changes, were selected for this study (Figure 1).

The Senegal River basin, which covers approximately $300000 \mathrm{~km}^{2}$ at the Diama station, is characterized by irregular rainfall, with a decreasing South-North gradient. The basin is divided into the Sahelian zone in the North and the Sudanian and Guinean zones in the South. The main inflow to the Senegal River comes from the Fouta Djalon Mountains, where rainfall can exceed $2000 \mathrm{~mm} / \mathrm{year}$. Precipitation in the north of the basin is less than $200 \mathrm{~mm} /$ year (Andersen et al., 2001).

The Nakambe basin is located in the upper part of the Volta basin in Burkina Faso (between $14^{\circ} 1^{\prime} \mathrm{N}$ $10^{\circ} 9^{\prime} \mathrm{N}$ and $\left.2^{\circ} 5^{\prime} \mathrm{W}-0^{\circ} 1^{\prime} \mathrm{E}\right)$. The climate is SudanoSahelian with a mean annual precipitation of around $700 \mathrm{~mm}$. The basin area is $40836 \mathrm{~km}^{2}$ at Bagre station.

\section{Datasets and models}

The seasonal cycle of simulated rainfall was compared to the gridded observations by the Climate Research Unit (CRU, University of East Anglia, Norwich, UK) and Institut de Recherche pour le D'evelopment (IRD) rainfall datasets. The IRD dataset corresponds to onsite daily rainfall data, interpolated for a regular 


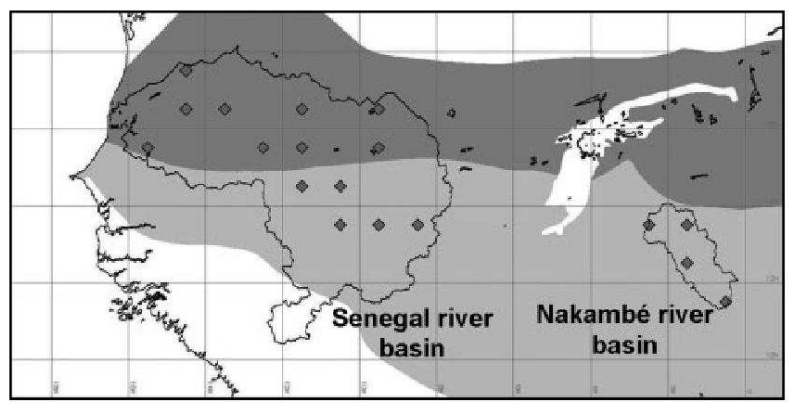

Figure I. Selected points on both Senegal River basin and Nakambe River basin. Dark gray area represents the Sahelian Acacia Savanna (SAS), and light gray area the West Sudanian Savanna (WSS) (according to the definition of eco-regions in Olson et al., 200I).

grid of $1^{\circ}$ (Diedhiou et al., 1999): it has been used extensively (Janicot and Sultan, 2001; Messager et al., 2004). The CRU dataset is a long-term climatology dataset that covers Africa, with a regular $0.5^{\circ}$ grid. In addition, for the Nakambe basin, historical data from national stations were used for precipitation and potential evapotranspiration (PET) (1961-2004) and discharges (1955-2008 at the Wayen station).

RCM simulations from the ENSEMBLES and IMPETUS projects were also considered for climate variability analysis. The global climate models (GCMs) cannot account for the regional heterogeneity of climate variability and change; therefore they are not suitable in order to produce the fine-scale climate projections needed to assess impacts (Paeth et al., 2011). Dynamical downscaling, such as RCMs, can be used to undertake this issue. The RACMO KNMI and REMO MPI (Paeth et al., 2005) models (both driven by ECHAM5 GCM), and the RCA SMHI RCM (driven by HadCM3 GCM), were selected for this study.

\subsection{Non-stationary analysis}

In order to analyze trends in the frequency distributions of average annual precipitation and maximum length of dry spells, the Generalized Additive Models for Location Scale and Shape (GAMLSS) technique was used, considering four different twoparameter distribution (Gumbel-GU-, Gamma-GA, Lognormal-LN-, and Weibull-WEI-). According to Rigby and Stasinopoulos (2005), GAMLSS are semi-parametric regression models. GAMLSS allows the modeling of pdf parameters of response variables (annual rainfall amount or DSL) as a function of an explanatory variable (time) through nonparametric smoothing functions. Hence, GAMLSS is a powerful tool to analyze hydrological time series which have non-stationary behavior (Villarini et al., 2009). A detailed description about the GAMLSS hypothesis, model selection, and examples can be found in Rigby and Stasinopoulos (2005).

Copyright $\odot 2011$ Royal Meteorological Society

\subsection{Hydrological model}

For hydrological modeling of the Nakambe basin, the GR2M model was employed (Maklouf, 1994). It is a conceptual, semi-distributed $\left(0.5^{\circ} \times 0.5^{\circ}\right)$ and monthly model with two parameters. This model has been extensively used for hydrological modeling of Sahelian basins (Ouedraogo, 2001; Mahé et al., 2005; Diello, 2007; Ardoin-Bardin et al., 2009). The analysis of the impacts of climate variability and change on runoff and water resources was carried out on the Nakambe basin. The GR2M hydrological model was calibrated and validated using historical observed data from the IRD and synoptic stations. Once calibrated and validated, the model was forced by the three RCMs' future climate dataset (RACMO, $\mathrm{RCA} / \mathrm{SMHI}$ and REMO). In this study, we do not consider land use change scenarios, which means model parameters remain constant over simulations. The PET was calculated using the Makkink method (Xu and Singh, 2002) from climate variables provided by the RCMs. The PET and precipitation data from RCMs were bias corrected based on the monthly and annual averages of observations over the 1961-1990 period.

\section{Results and discussion}

\section{I. Analysis of rainfall seasonal cycle}

In order to carry out a regional analysis, the selected basins were subdivided according to eco-regions defined by Olson et al. (2001). These eco-regions are the Sahelian Acacia Savanna (SAS) and the West Sudanian Savanna (WSS) (Figure 1). Since the spatial distribution of the IRD dataset does not constitute a continuous grid, 14 cells in the Senegal River basin ( 9 cells in SAS and 5 cells in WSS) and four cells in the Nakambe river basin were selected for the analysis. The seasonal cycle of rainfall over the 1970-1990 period from the IRD, CRU and simulated rainfall datasets, for the selected eco-regions, was estimated

Atmos. Sci. Let. 12: 109-1 I5 (201 I) 

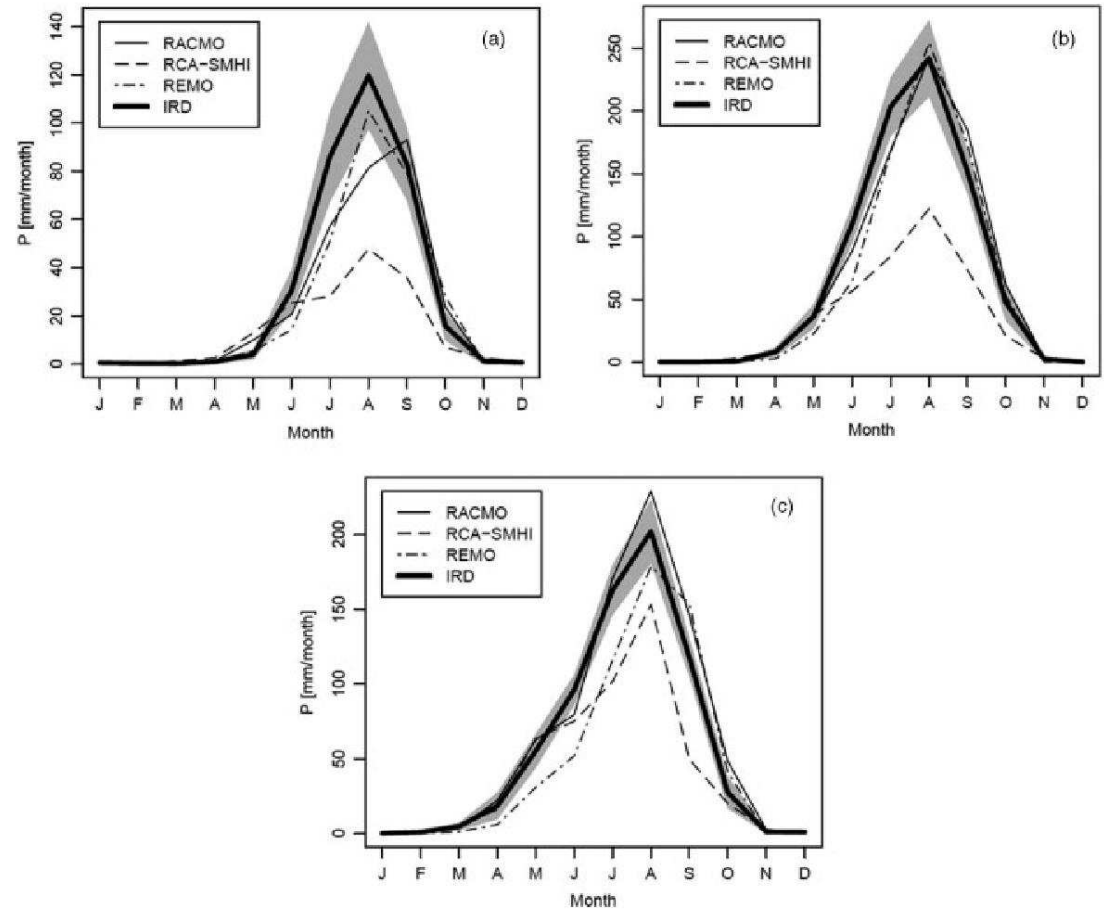

Figure 2. Analysis of regional seasonal cycle of rainfall over the period 1970-1990. (a) Senegal basin SAS eco-region, (b) Senegal basin WSS eco-region and (c) Nakambe basin. Gray area corresponds to $95 \%$ confidence interval.

Table I. Analysis of the abilities of selected RCMs to represent the seasonal cycle of rainfall (time period 1970-1990).

\begin{tabular}{lrrrrr}
\hline & & & \multicolumn{3}{c}{$\begin{array}{c}\text { PMEAN } \\
\text { (mm/year) }\end{array}$} \\
\cline { 3 - 6 } & & & & IRD & CRU \\
\hline Senegal basin SAS & $14.9 \%$ & $30.6 \%$ & $12.7 \%$ & 342.3 & 314.8 \\
Senegal basin WSS & $6.8 \%$ & $24.4 \%$ & $8.2 \%$ & 803.0 & 723.4 \\
Nakambe basin & $7.1 \%$ & $15.5 \%$ & $12.1 \%$ & 687.1 & 692.9 \\
\hline
\end{tabular}

and compared (Table I and Figure 2). The good fit between IRD and CRU is observed (in general, the CRU monthly rainfall cycle falls into the $95 \%$ confidence interval assessed with IRD data).Compared to observations, the RCMs differ in how they reproduce the seasonal cycle, due mainly to their different dynamical schemes and physical parameterizations of the West African monsoon (Paeth et al., 2011). For both basins, the RACMO model seems to present a better ability.

\subsection{Analysis of DSL}

Dry spells in the monsoon season were identified and analyzed. The DSL was defined as the number of consecutive days with rainfall of less than
$1 \mathrm{~mm} /$ day between 1 May and 30 September over the period 1970-1990. However, some dry spells could have days corresponding to both the monsoon season and the offseason. In this case, only the days in the monsoon season were considered in the definition of the DSL. Therefore, monsoon dry spells that belong to several years could be presented. The basic statistics of the DSL (mean $\mu_{\mathrm{DSL}}$ and standard deviation $\sigma_{\mathrm{DSL}}$ ) were estimated from the daily IRD dataset and the RCMs, for the selected sites. The root mean square error (RMSE) of these statistics, as a percentage of IRD values, was calculated (Table II). The mean DSL in the Sahelian region (SAS) is double that in the Sudanian region. The variability of DSL $\left(\sigma_{\mathrm{DSL}}\right)$ is also greater in the SAS region.

In general, the mean DSL $\left(\mu_{\mathrm{DSL}}\right)$ is better represented by the RACMO model, followed by REMO and RCA/SMHI models. According to the different eco-regions defined, the REMO model presents a better ability to simulate DSL for the Senegal basin SAS eco-region, while the RACMO model shows good behavior in the Senegal basin WSS eco-region and the Nakambe basin. The RCA/SMHI exhibits poor fit of $\mu_{\mathrm{DSL}}$ and $\sigma_{\mathrm{DSL}}$ in all regions considered (Table II). 

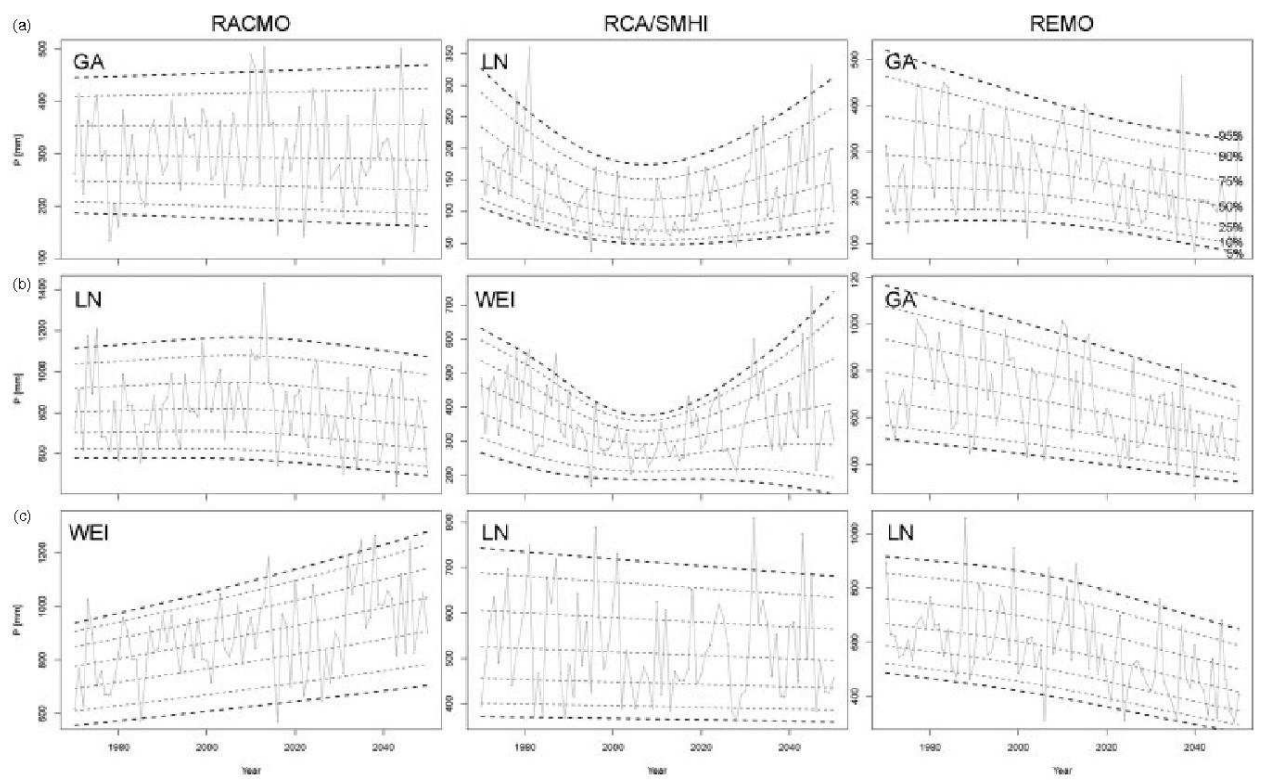

Figure 3. GAMLSS analysis of annual rainfall using RCMs: (a) Senegal basin SAS, (b) Senegal basin WSS and (c) Nakambe basin. The centile curves $(5-95 \%)$ are shown by dashes.

Table II. Analysis of abilities of selected RCMs to represent the statistics of DSL (time period 1970-1990).

\begin{tabular}{llll}
\hline RMSE related & & & \\
with DSL & RACMO RCAISMHI REMO IRD
\end{tabular}

with DSL

RMSE of mean of

DSL $\mu$

Senegal basin SAS

Senegal basin

WSS

Nakambe basin

RMSE of standard

deviation of DSL

$\begin{array}{lllll}\text { Senegal basin SAS } & 46.0 \% & 32.6 \% & 13.3 \% & 121\end{array}$

$\begin{array}{lrrrr}\text { Senegal basin } & 7.9 \% & 22.4 \% & 53.0 \% & 3.1\end{array}$

WSS

$\begin{array}{lllll}\text { Nakambe basin } \quad 17.3 \% & 24.7 \% & 73.7 \% & 2 .\end{array}$

\subsection{Analysis of future trends Changes in annual precipitation}

Applying GAMLSS, we calculated the time variation of the frequency distribution of yearly precipitation. Figure 3 presents the regional results obtained with the different RCMs. The centiles (5-95\%) which show the time evolution of the fitted model from GAMLSS are also presented. Table III presents the annual precipitation changes between 1990 and 2050 . The RACMO model predicts a slight decrease in the amount of rainfall for the Senegal basin, and an increment for the Nakambe basin, while the REMO model predicts a decrease in the amount of annual

Copyright $\odot$ 201I Royal Meteorological Society rainfall (about $30 \%$ ) in all regions. The RCA/SHMI model shows a strong increase trend (about 25-30\%) on the Senegal basin and a small decrease in the Nakambe basin. The RCMs do not converge on future precipitation changes in Western Africa. As mentioned below, this is mainly due to model errors and processes representation in RCMs, rather than to differences in driving GCMs (Paeth et al., 2011).

\section{Changes in the annual maximum DSL in the monsoon} season

In each region, the time series of the annual maximum DSL for each year (AMDSL) for the monsoon season were built, taking into account the maximum dry spell in the year considering all selected sites. Table III shows the AMDSL changes between 1990 and 2050. An increase of AMDSL is generally predicted. Our findings are coherent with the results of García Galiano and Giraldo Osorio (2010), working with REMO ensemble members and empirical cumulative density function on the Senegal River basin. For the RACMO and REMO models, the increase on the Senegal basin SAS eco-region is projected at between 3 and $25 \%$ ( $3-35$ days), whereas on the Senegal basin WSS eco-region the estimated increment is about $12-36 \%$ (6-12 days). On the Nakambe basin an increase of AMDSL between 16 and $47 \%$ (3-18 days) is projected by both RACMO and REMO. Only the RCA/SMHI model shows a decrease of the AMDSL on the Senegal basin (between 15 and 24\%). 


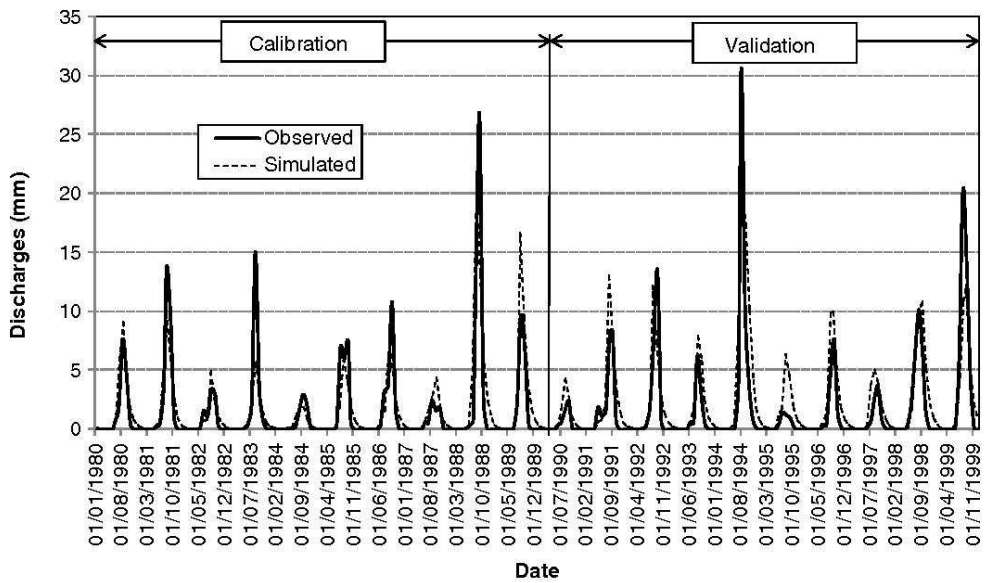

Figure 4. Comparison between simulated and observed hydrographs in calibration and validation of the GR2M model on the Nakambe basin.

Table III. Projected values for 2050 against the 1990 value for each RCM. The IRD values have been estimated for the period 1970-1990.

\begin{tabular}{|c|c|c|c|c|c|c|c|}
\hline & \multicolumn{2}{|c|}{ RACMO } & \multicolumn{2}{|c|}{ RCASMHI } & \multicolumn{2}{|c|}{ REMO } & \multirow{2}{*}{$\begin{array}{c}\text { IRD } \\
1970-1990\end{array}$} \\
\hline & 1990 & 2050 & 1990 & 2050 & 1990 & 2050 & \\
\hline \multicolumn{8}{|c|}{ Mean annual precipitation (mrn) } \\
\hline Senegal basin SAS & 303.1 & 298.5 & 123.5 & 1632 & 458.0 & 352.3 & 342.3 \\
\hline Senegal basin WSS & 834.2 & 748.6 & 340.4 & 422.7 & 741.8 & 509.8 & 803.0 \\
\hline Nakambé basin & 822.9 & 1018.0 & 529.2 & 505.0 & 640.9 & 431.5 & 687.1 \\
\hline \multicolumn{8}{|c|}{ Annual maximum DSL-95\% centile (day) } \\
\hline Senegal basin SAS & 92.3 & 95.1 & 171.2 & 146.3 & 138.6 & 173.6 & 180.3 \\
\hline Senegal basin WSS & 327 & 44.4 & 43.3 & 330 & 55.9 & 62.5 & 48.7 \\
\hline Nakambé basin & 21.4 & 24.8 & 19.3 & 19.7 & 39.3 & 57.8 & 31.5 \\
\hline
\end{tabular}

\subsection{Analysis of impacts on runoff}

The hydrological model GR2M calibration was performed for the period 1980-1989 with a Nash performance coefficient (Nash and Sutcliffe, 1970) of $72 \%$. The model validation was done using the 1990-1999 period, and the results were quite similar to those obtained by the calibration process (Nash coefficient of $71 \%$ ). The good fit between simulated and observed hydrographs in calibration and validation is shown in Figure 4.

An extended analysis of the historical discharges on the Nakambe River basin was carried out by Mahé et al. (2005) and Mahé and Paturel (2009). These previous studies showed that conversely to rainfall decrease, river discharges increased strongly in this basin after the 1970 s compared to the period before 1970. This increase reached $193 \%$ for the period 1974-2008 compared to the period 1955-1973. In the last decade 1991-2000, discharges were 2.2 times higher than before 1970 (Figure 5).

With regard to precipitation, future trends of river discharges differ from one RCM to another. Compared

Copyright $\odot 2011$ Royal Meteorological Society to the decade 1991-2000, the RACMO and REMO models predict an increase of discharges for coming decades. This increase goes up to $156 \%$ during the decade 2031-2040 for RACMO and 68\% during 2011-2020 for REMO. The RCA/SMHI model shows first a decrease of discharges (up to 17\%) for the two decades 2011-2020 and 2021-2030, and then an increase (up to $36 \%$ ) for the decades 2031-2040 and 2041-2050, compared to 1991-2000. These trends in river discharges are not in concordance with climate forcing variables. In fact, compared to the decade 1991-2000, the PET shows the same magnitude of decrease $(1-5 \%)$ for all the RCMs (not shown). In the case of precipitation, trends differ between the models. The RCA/SMHI and REMO models show a decreasing trend of rainfall for future decades, while the RACMO model predicts a general increase trend compared to the decade 1991-2000 (not shown). If this increase of precipitation predicted by the RACMO model induces an increase in river discharges, the decrease of precipitation indicated by RCA/SMHI and 


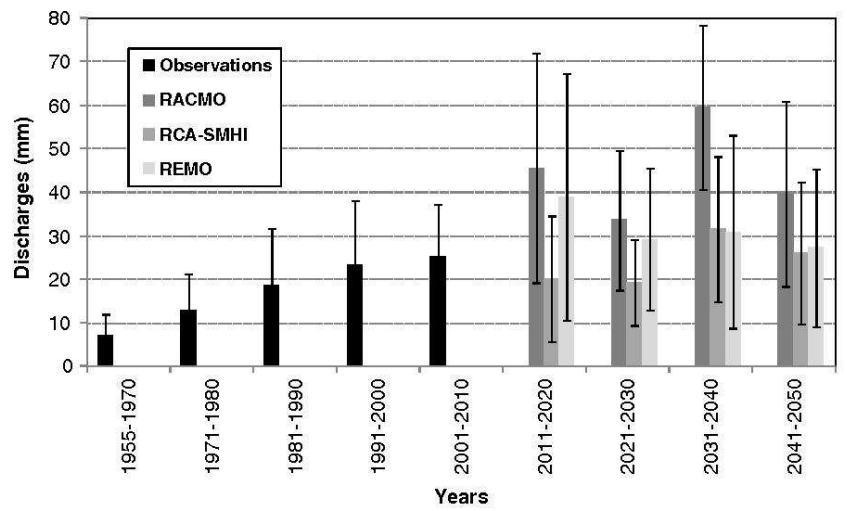

Figure 5. Evolution of decadal discharges (observed and predicted) on the Nakambe basin.

REMO will result in a general increase of river flows (mainly for REMO).

These results highlight the difficulty in predicting the response of hydrosystems in this semi-arid region. A decrease in precipitation will not necessarily induce a decrease in runoff and river discharges. This has already been demonstrated by observations (Mahé et al., 2005). Several authors attributed this contradictory hydrological response of Sahelian catchments to land use changes (Leduc et al., 2001; Favreau et al., 2009; Mahé and Paturel, 2009; Séguis et al., 2011) and the extension of hydrographic networks (Leblanc et al., 2008). Indeed, the increase of crusted and cultivated areas to the detriment of natural vegetation cover since the 1960s has led to a decrease of soil infiltration capacity and an enhancement of surface runoff (Mahé et al., 2005). Thus, in addition to reducing errors in RCMs and building reliable climate predictions in West Africa for impact assessment, another remaining challenge resides in developing land use scenarios and integrating them into hydrological modeling. Future research actions should be oriented in this direction in order to develop reliable decision support tools enabling better adaptation to climate variability and change in the Sahel.

\section{Conclusion}

From observed and RCMs data, plausible trends in rainfall and frequency of dry spells and mean annual rainfall are identified and analyzed on two West African river basins (Senegal and Nakambe). A nonstationary behavior of the annual series of maximum of DSL in the monsoon season is reflected in temporal changes in mean and variance. Impacts analysis on runoff in the Nakambe basin was also undertaken.

From the comparison of RCMs selected against observed rainfall dataset, we conclude that the RACMO model presents the best ability to represent the seasonal cycle of precipitation, particularly in the WSS eco-region of both basins. REMO appears better to represent the seasonal cycle in the Senegal basin SAS eco-region. Also, RACMO represents the DSL distribution better than the other models, whereas REMO adequately estimates the mean of DSL, but it is not satisfactory with standard deviation. Finally, the RCA/SMHI model does not present good behavior in the simulation of analyzed variables (precipitation or DSL).

For 2050, a decrease of annual precipitation projected is observed for both RACMO and REMO models in all regions (except in the Nakambe basin, where RACMO predicts an increase in annual rainfall). On the other hand, the AMDSL 2050 projection from the RACMO and REMO model presents an increase, especially in the WSS eco-region of both the Senegal and Nakambe basins.

Impact assessment on water resources on the Nakambe River basin generally show an increase trend of discharges for coming decades compared to 1991-2000. These trends in river flows highlight the influence of land uses and covers in analyzing hydrological response and water resources availability in the Sahel. In order to generalize these results, the analysis should be extended to other basins in the region and more RCM outputs from ENSEMBLES should be utilized. A remaining challenge which can guide future research on assessing climate change impacts on water resources in the Sahel will be the elaboration of land use scenarios to be integrated in hydrological models.

\section{Acknowledgments}

The work has been supported by the EU FP6 AMMA project. The support of the AMMA project is gratefully acknowledged (see http://onlinelibrary. wiley.com/doi/10.1002/asl.331/full for full acknowledgement). 


\section{References}

Andersen J, Refsgaard J, Jensen KH. 2001. Distributed hydrological modelling of the Senegal river basin - model construction and validation. Journal of Hydrology 247: 200-214.

Ardoin-Bardin S, Dezetter A, Servat E, Paturel JE, Mahé G, Niel H, Dieulin C. 2009. Using general circulation model outputs to assess impacts of climate change on runoff for large hydrological catchments in West Africa. Iydrological Sciences Journal 54(1): catchme.

Descroix L, Mahé G, Lebel T, Favreau G, Galle S, Gautier E, Olivry J-C, Albergel J, Amogu O, Cappelaere B, Dessouassi R, Diedhiou A, Le Breton E, Mamadou I, Sighomnou D. 2009. Spatiotemporal variability of hydrological regimes around the boundaries between Sahelian and Sudanian areas of West Africa: a synthesis. Joumal of Hydrology 375: 90-102

Diedhiou A, Janicot S, Viltard A, de Félice P, Laurent H. 1999. Easterly waves regimes and associated convection over West Africa and the tropical Atlantic: Results from the NCEP/NCAR and ECMWF reanalyses. Climate Dynarnics 15: 795-822.

Diello P. 2007. Interrelations Climat - Homme - Environnement dans Diello P. 2007. Interrelations Climat - Homme - Enviromnement dans
le Sahel Burkinabé: impacts sur les états de surface et la modélisation le Sahel Burkinabé: impacts sur les états de surface et la modélisation
hydrologique. $\mathrm{PhD}$ thesis, University of Montpellier II, Montpellier, France; 368 .

Favreau G, Cappelaere B, Massuel S, Leblanc M, Boucher M, Boulain N, Leduc C. 2009. Land clearing, climate variability, and water resources

Gareía Galiano SG, Giraldo Osorio JD. 2010. Analysis of impacts García Galiano SG, Giraldo Osonio JD. 2010. Analysis of impacts
on hydrometeorological extremes in the Senegal River Basin from on hydrometeorological extremes in the Senegal River Basi
REMO RCM. Meteorologische Zeitschrift 19(4): 375-384.

Janicot S, Sultan B. 2001. Intra-seasonal modulation of convection in the West African monsoon. Geophysical Research Letters 28(3): 523-526.

Leblane M, Favreau G, Massuel S, Tweed S, Loireau M, Cappelaere B. 2008. Land clearance and hydrological change in the Sahel: SW Niger Global and Planetary Change 61: 135-150.

Niger. Global and Planetary Change 61: 135-150.
Leduc C, Favreau G, Schroeter P. 2001. Long term rise in a Sahelian watertable: the continental terminal in South-West Niger. Journal of

Maklouf Z. 1994. Compléments sur le modèle pluie-débit GR4J et essai d'estimation de ses paramètres. $\mathrm{PhD}$ thesis, University of Paris XI Orsay; 426

Mahé G. Paturel JE. 2009 1896-2006 Sahelian annual rainfall variability and runoff increase of Sahelian Rivers. CR. Geosciences 341: $538-546$.

Mahé G. Paturel JE, Servat E, Conway D. Dezetter A. 2005. Impact of land use change on soil water holding capacity and river modelling of the Nakambe River in Burkina-Faso. Journal of Hydrology 300: 33-43.
Messager G, Gallée $H$, Brasseur 0 . 2004. Precipitation sensitivity to regional SST in a regional climate simulation during the West African monsoon for two dry years. Climate Dynamics 22: $249-266$.

Nash JE, Sutcliffe JV, 1970. River flow forecasting through conceptual models. Part I - a discussion of principles. Journal of Hydrology 10 ; 282-290.

Olson DM, Dinerstein E, Wikramanayake ED, Burgess ND, Powell GVN, Underwood EC, D'amico JA, Itoua I, Strand HE, Morrison JC, Loucks CJ, Allnutt TF, Ricketts TH, Kura Y, Lamoreux JF Wettengel WW, Hedao P, Kassem KR. 2001. Terrestrial Ecoregions of the World: A New Map of Life on Earth. BioScience 51(11): $933-938$.

Ouedraogo M. 2001. Contribution à l'étude de l'impact de la variabilité climatique sur les ressources en eau en Afrique de l'Ouest. Analyse des conséquences d'une sécheresse persistante : normes hydrologiques et modélisation régionale. $\mathrm{PhD}$ thesis, University of Montpellier II, Montpellier (France); 257.

Paeth H, Born K, Podzun R, Jacob D, 2005. Regional dynamical downscaling over West Africa: model evaluation and compaison of wet and dry years Meteorologische Zeitschrift 14(3): 349-367.

Paeth H, Hall NMJ, Gaertmer MA, Dornínguez-Alonso M, Moumouni S, Polcher J, Ruti PM, Fink AH, Gosset M, Lebel T, Gaye A, Rowell DP, Moufouma-Okia W, Jacob D, Rockel B, Giorgi F, Rumunukainen M. 2011. Progress in regional downscaling of West African precipitation. Atmospheric Science Letters 12: 75-82, DOI 10.1002/as1.306.

Polcher J, Parker DJ, Gaye A, Diedhiou A, Eymard L, Fierli F, Genesio L, Höller H, Janicot S, Lafore J-P, Karambiri H, Lebel T, Redelsperger J-L, Reeves CE, Ruti P, Sandholt I Thorncroft C Redelsperger J-L, Reeves $C E$, Ruti $\mathrm{P}$, Sandholt $\mathrm{I}$, Thorncroft $\mathrm{C}$.
2011. AMMA's contribution to the evolution of prediction and 2011. AMMA's contribution to the evolution of prediction and
decision-making systems for West Africa. Atmospheric Science Letters 12: 2-6, DOI: $10.1002 /$ asl.320.

Rigby RA, Stasinopoulos DM. 2005. Generalized additive models for location scale and shape. Applied Statistics 54: 507-554.

Seguis L, Boulain N, Cappelaere B, Cohard JM, Favreau G, Galle S, Guyot A, Hiernaux P, Mougin E, Peugeot C, Ramier D, Seghieri J, Guyot A, Hiernaux P, Mougin E, Peugeot C, Ramier D, Seghieri J, Timouk F, Demarez V, Demarty J, Descroix L, Descloitres M, Grippa M, Guichard F, Kamagate BS, Kergoat L, Lebel T,
Le Dantec V, Le Lay M, Massuel S, Trichon V. 2011. Contrasted land surface processes along the West African rainfall gradient. Atmospheric Science Letters 12: 31-37, DOI: 10.1002/as1.327.

Villarini G, Smith JA, Serinaldi F, Bales J, Bates PD, Krajewski WF. 2009. Flood frequency analysis for nonstationary annul peak records in an urban drainage basin. Advances in Water Resources 32(8): $1255-1266$.

Xu C-Y, Singh VP. 2002. Cross comparison of empirical equations for calculating potential evapotranspiration with data from Switzerland. Water Resources Management 16: 197-219. 



\subsection{Resumen del Artículo 3}

\subsubsection{Título}

Building hazard maps of extreme daily rainy events from PDF ensemble, via REA method, on Senegal River Basin.

\subsubsection{Objetivos específicos}

- Implementar una metodología objetiva para evaluar la confiabilidad de los diferentes RCMs (6) considerados, provistos por el Proyecto Europeo ENSEMBLES, en la modelización de eventos extremos anuales de precipitación diaria.

- Construir mapas que reflejen esta confiabilidad para cada modelo climático, en la Cuenca del Río Senegal.

- Analizar la tendencia futura de las precipitaciones máximas en cada sitio de análisis, ajustando distribuciones de probabilidad que permitan una simulación no estacionaria.

- Analizar el patrón espacio-temporal de la tendencia en la distribución de las precipitaciones máximas, construyendo mapas interpolados de estadísticos seleccionados para diferentes horizontes de pronóstico.

- Proveer de evaluaciones cuantitativas de las incertidumbres asociadas, a ser consideradas por los responsables de toma de decisión en aspectos de gestión de recursos hídricos a escala de cuenca hidrográfica. Para alcanzar un mejor balance entre mitigación y adaptación al cambio y variabilidad climática en la región.

\subsubsection{Metodología}

La zona de estudio corresponde a la Cuenca del Río Senegal, la cual es compartida por Guinea, Malí, Senegal y Mauritania. La OMVS (Organization pour la Mise en Valeur du fleuve Sénégal) es la organización transnacional que se encarga de la administración del río y su cuenca de drenaje.

En este estudio se utilizaron mallas interpoladas de datos de precipitación diarios observados, recogidos por el IRD. Además, datos simulados diarios de precipitación desde seis RCMs fueron seleccionados, distribuidos por el proyecto europeo ENSEMBLES (Christensen et al., 2009). La selección de los RCMs se basó en su coincidencia temporal con los datos IRD (que tienen datos entre 1970-1990), que permitió llevar a cabo el análisis del desvío. Se seleccionaron 120 sitios de análisis (o grid-sites) sobre una malla regular $1^{\circ} \times 1^{\circ}$. Sin embargo, la discontinuidad espacial de los datos IRD restringió el análisis del desvío a 43 de estos sitios. Las series 
temporales de precipitación diaria máxima anual (AMDR) desde los datos simulados y observados, se obtuvieron en los sitios definidos anteriormente.

Diversos estudios han demostrado divergencia considerable entre los modelos climáticos al simular extremos hidrológicos (García Galiano and Giraldo Osorio, 2010; Karambiri et al., 2011). Debido a que no es posible concluir sobre cuál de todos los modelos es el más confiable, se concluye que los pronósticos deben basarse en un ensemble de todos los RCMs. Debido a la no estacionariedad de las series temporales obtenidas, se utilizó la herramienta GAMLSS (Rigby and Stasinopoulos, 2005; Stasinopoulos and Rugby, 2007) para el ajuste de distribuciones de probabilidad no estacionaria a las series de precipitación máxima en cada sitio.

El método REA (Giorgi and Mearns, 2002), permite estimar la incertidumbre del cambio simulado con un ensemble de diferentes modelos climáticos. El método considera dos criterios para estimar el factor de confiabilidad $R$. El primero de ellos es el criterio de desempeño $\left(R_{B}\right)$, que tiene en cuenta el desvío de cada modelo respecto de los datos observados. El segundo criterio es el criterio de convergencia $\left(R_{D}\right)$, que evalúa el desvío de cada modelo respecto "del mejor estimado" para el clima futuro. Para tener un alto valor de confiabilidad, ambos criterios deben cumplirse, ya que $R=$ $R_{B} \cdot R_{D}$.

Para la estimación de $R_{B}$ y $R_{D}$ se utilizó la métrica propuesta por Perkins ( $S_{S C O R E}$; Perkins et al., 2007). El $S_{S C O R E}$ es una medida simple del grado de acuerdo entre dos distribuciones de probabilidad, ya que estima el área común entre dos PDF. En el caso de $R_{B}$, su cálculo ha sido relativamente sencillo, ya que se dispone de la distribución de probabilidad de los datos observados. Sin embargo, para $R_{D}$ existe la dificultad de no conocer la distribución "del mejor estimado" del clima futuro, por lo que un proceso iterativo ha sido ejecutado para obtener el valor de $R_{D}$.

Los valores de los diferentes factores REA $\left(R, R_{B}, R_{D}\right)$ han sido estimados en los sitios donde existían datos IRD. Se obtuvieron mapas interpolados de estos factores para cada uno de los modelos miembros del ensemble. Utilizando álgebra de mapas, se calcularon los mapas de $\mathrm{Pm}$ (el factor de confiabilidad normalizado), que se puede interpretar como la probabilidad asociada con cada RCM. Los mapas de $P m$ han permitido la construcción de las PDF ensembles en los sitios donde no existían datos IRD.

Como ya se ha comentado, el ajuste GAMLSS a las series temporales de cada modelo en cada sitio predijo tendencias que difieren en magnitud y signo. Además, se ha calculado el valor Pm en cada sitio de análisis. En consecuencia, para construir la PDF ensemble para cada sitio, un peso ponderado más alto fue otorgado a los modelos con 
valores más elevados del factor de confiabilidad, utilizando técnicas de bootstrapping (Efron and Tibshirani, 1993). Para obtener la evolución de la distribución, la PDF ensemble se construyó cada diez años en el periodo 1970-2050, calculando los estadísticos básicos (media y desviación estándar), los cuantiles del 5, 10, 25, 50, 75, 90 y $95 \%$ y, para todos ellos, los intervalos de confianza del $95 \%$,

\subsubsection{Resultados}

Obtenidos las PDF ensemble en los sitios de análisis, sus valores fueron interpolados para generar mapas que permitieran observar la evolución espacio-temporal de la distribución de las AMDR.

Los mapas interpolados para los diferentes estadísticos (para 1990 como año de referencia, y para el horizonte de pronóstico 2050), preservan el gradiente latitudinal de la precipitación promedio anual, sin embargo, la diferencia entre ambos no conserva esta estructura. Se espera un aumento significativo en el valor medio de las AMDR en el Valle del Río Senegal y en la parte alta de la cuenca, pero en la zona del Sahel se proyecta un descenso, aunque no significativo. En cuanto a la desviación estándar de las AMDR, se espera un aumento significativo en la parte baja de la cuenca, y no significativo en la parte alta de la misma.

Finalmente, se espera un aumento generalizado de las AMDR asociadas con los cuantiles 90 y 95\% (periodos de retorno 10 y 20 años, respectivamente). El aumento se prevé significativo en la parte baja de la cuenca (zona del Valle del Río Senegal).

\subsubsection{Conclusiones y recomendaciones}

Una novedosa aproximación con PDF ensemble se ha presentado en este trabajo, que ha permitido la identificación de tendencias espacio-temporales plausibles en la distribución de las AMDR. Desde los datos de los RCM, se ha demostrado que el calentamiento global afectará la frecuencia e intensidad de los eventos de precipitación extrema en la Cuenca del Río Senegal. El impacto sobre la sociedad dependerá no sólo de la intensidad del evento, sino también de la habilidad de la población para adaptarse a las nuevas condiciones. El conocimiento y entendimiento para adoptar las medidas adecuadas, es importante.

Con el fin de aprovechar toda la información de los RCM, se construyeron PDF ensemble para cada sitio de análisis en la zona de estudio, lo que permitió la representación de los cambios espacio-temporales de las AMDR.

Para cada sitio, se capturó la no estacionariedad de la distribución de probabilidad para cada modelo utilizado GAMLSS. Luego, estas distribuciones ajustadas fueron 
combinadas en la PDF ensemble teniendo en cuenta el diferente desempeño de cada modelo.

Los mapas interpolados para los diferentes estadísticos conservan el gradiente latitudinal característico de la precipitación. Sin embargo, los mapas de diferencia para los años considerados (1990 y 2050) se alejan de este patrón espacial. Estos mapas de diferencia muestran que el aumento de las precipitaciones extremas será significativo en la parte baja de la cuenca, mientras que en la cuenca alta se presentará un aumento menor en magnitud y no significativo.

Se proponen como líneas futuras de investigación análisis de otros métodos de estimación de los factores de confiabilidad de cada RCM del ensemble, con el fin de verificar la consistencia espacio-temporal de los resultados entre las diferentes aproximaciones metodológicas, así como evaluar la incertidumbre asociadas al incluir nuevas variables y zonas con otras características hidroclimáticas. Otra línea de investigación a considerar, es estudiar si la mejora en la resolución espacial de los modelos climáticos afectará las conclusiones que hasta ahora se han alcanzado. 


\title{
5.3.6 Copia de la publicación
}

Hydrol. Earth Syst. Sci., 15, 3605-3615, 2011 www.hydrol-earth-syst-sci.net/15/3605/2011/ doi: $10.5194 /$ hess-15-3605-2011

(C) Author(s) 2011. CC Attribution 3.0 License.

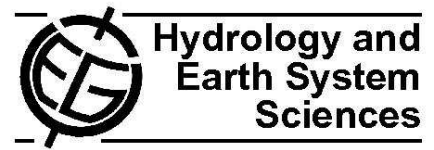

\section{Building hazard maps of extreme daily rainy events from PDF ensemble, via REA method, on Senegal River Basin}

\author{
J. D. Giraldo Osorio and S. G. García Galiano \\ Universidad Politécnica de Cartagena, Department of Civil Engineering, R\&D Group of Water Resources Management, \\ Paseo Alfonso XIII, 52, 30203, Cartagena, Spain
}

Received: 23 March 2011 - Published in Hydrol. Earth Syst. Sci. Discuss.: 15 April 2011

Revised: 2 November 2011 - Accepted: 16 November 2011 - Published: 29 November 2011

\begin{abstract}
The Sudano-Sahelian zone of West Africa, one of the poorest of the Earth, is characterized by high rainfall variability and rapid population growth. In this region, heavy storm events frequently cause extensive damage. Nonetheless, the projections for change in extreme rainfall values have shown a great divergence between Regional Climate Models (RCM), increasing the forecast uncertainty. Novel methodologies should be applied, taking into account both the variability provided by different RCMs, as well as the non-stationary nature of time series for the building of hazard maps of extreme rainfall events. The present work focuses on the probability density functions (PDFs)-based evaluation and a simple quantitative measure of how well each RCM considered can capture the observed annual maximum daily rainfall (AMDR) series on the Senegal River basin. Since meaningful trends have been detected in historical rainfall time series for the region, non-stationary probabilistic models were used to fit the PDF parameters to the AMDR time series. In the development of PDF ensemble by bootstrapping techniques, Reliability Ensemble Averaging (REA) maps were applied to score the RCMs. The REA factors were computed using a metric to evaluate the agreement between observed -or best estimated- PDFs, and that simulated with each RCM. The assessment of plausible regional trends associated to the return period, from the hazard maps of AMDR, showed a general rise, owing to an increase in the mean and the variability of extreme precipitation. These spatialtemporal distributions could be considered by Organization for the Development of the Senegal River (Organisation pour la mise en valeur du fleuve Sénégal, OMVS), in such a way as to reach a better balance between mitigation and adaptation.
\end{abstract}

Correspondence to: S. G. García Galiano (sandra.garcia@upct.es)

\section{Introduction}

One of the areas hardest hit by the twentieth century climate change impacts, has been the densely populated Sahel (West Africa). Over the period 1990-2020, its population is expected to double, with projections of even higher growth for some cities in the Senegal River Valley (Cour, 2001). The climatic fluctuations in the Sahel have been devastating (famines, migrations, and slow economic growth), indicating the great vulnerability of the region (Lebel et al., 2009; Paeth et al., 2009). The study of the continuous rise in greenhouse gases emission, and the associated response of the climate, has received more attention for the region in recent years. Global warming has caused changes in rainfall patterns and, consequently, changes in the frequency and magnitude of extreme events (Labat et al., 2004; Huntington, 2006; Kundzewicz et al., 2007). Huntington (2006) points out that the evidence about the current and future intensifying of the hydrologic cycle is robust, and highlights the need to improve the ability of monitoring and predicting the impacts associated with the change of hydrologic regimes. In these conditions, the presumption of hydroclimatic stationarity cannot be guaranteed, due to the interaction of various drivers (land use and climate change, and population growth, among others). In concordance with the opinion of several authors (Milly et al., 2008; Villarini et al., 2010), nonstationary probabilistic models able to reproduce the variation with time of the parameters of selected probability density functions (PDFs) should be used.

In this sense, Regional Climate Models (RCMs) have become an important tool to improve the understanding of key processes involved in the description of climate mechanisms (Sánchez et al., 2009), for the simulation of plausible climate scenarios with an appropriate resolution for impact studies 
at basin scale. Several authors have worked with climate models to simulate the trends in extreme rainfall patterns, especially in Europe (Kharin and Zwiers, 2005; Kharin et al., 2009; Kyselý and Beranová, 2009; Nikulin et al., 2011, among others). There are notable advances in the simulation of dynamics of West Africa Monsoon (WAM) using climate models (Fontaine et al., 2011; Paeth et al., 2011; Ruti et al., 2011).

However, the RCMs built for West Africa are still sensitive to physical parameterizations, spatial-temporal resolution and internal variability (Paeth et al., 2011). The RCMs exhibit differing levels of skill over different regions and hydrometeorological variables, making it difficult to identify the models with greater confidence. As a consequence the climate projections and the estimation of uncertaintic associated are better based on the combination of information provided by an ensemble approach from different RCMs simulations (Giorgi and Mearns, 2002; Paeth et al., 2011). The model ensemble allows a more precise description of uncertainties and weaknesses, as well as a probabilistic approach to future climate projections (Sánchez et al., 2009). Tebaldi and Knutti (2007) conducted a review of methodologies addressing the building of model ensembles, comparing their results for regional temperature projections. However, the error obtained from combining multiple models is, sometimes, the result of error compensation. A weighting of models, based on observations, could ameliorate this problem (Sánchez et al., 2009). To assign weights to different RCMs of an ensemble, several statistics are usually compared considering observed data.

However, the use of statistics as means and standard deviations does not allow the comparison of the entire distribution of the data, even when the evidence shows those observed changes in the extreme events (tails) are different in magnitude to those observed in the mean values. In addition, the change associated with extreme values is expected to have a higher impact on biophysical systems (Perkins et al., 2007 Tapiador et a1., 2009). This situation justifies questioning the ability of RCMs to simulate the distribution of observed probability of the hydrometeorological variables. There are several works about the estimation of PDFs associated with several hydrometeorological variables from climate projections (Wigley and Raper, 2001; Giorgi and Mearns, 2003 Tebaldi et al., 2005; Giorgi, 2008; Buser et al., 2009). Establishing the best skill of a climate model for simulating the observed PDF of a variable implies that estimated projections with this model will have more confidence for future projections (Perkins et al., 2007; Sánchez et al., 2009). However the ability to represent the present-day climate well does not seem to be enough to asseverate the skill to simulate the future climate climate (Giorgi and Mearns, 2003).

With regard to the above, the present study focuses on the assessment of change in the PDF of the annual maximum daily rainfall (AMDR) on the Senegal River Basin (West Africa), considering the nonstationarity of time series and

Hydrol. Earth Syst. Sci., 15, 3605-3615, 2011 building at site PDF using a weighted ensemble. The RCMs and observational datasets considered for the target basin are described in Sect. 2.

Section 3 describes the implementation of the Reliability Ensemble Average (REA) method proposed by Giorgi and Mearns (2002), for assessing the ability of the RCMs to reproduce the present-day climate while at the same time evaluating the convergence of different RCMs to a given forcing scenario. The score method proposed by Perkins et al. (2007), is applied to identify the skill scores of the RCMs in the PDF ensemble for each site. Previous studies in the region have considered ensemble RCMs to assess impacts on extreme rainfall events (García Galiano and Giraldo Osorio, 2011). In the work of these authors, the reliability factors were considered constant for the entire study area, and estimated only based on the bias analysis (by SmirnovKolmorov test) between the observed and simulated series of AMDR (in other words, without consideration of convergence criteria between the time series to the future). In the present study, the REA factors are estimated spatially distributed.

Finally, the PDF ensembles were considered to build maps of various statistical values associated with the distribution of AMDR on the target basin, for studying the plausible trends of AMDR (Sect. 4). The PDF ensemble for each site allows to obtain a measure of the change uncertainty. The results and discussion section presents a brief analysis of the maps constructed, and the key findings of the work.

\section{Target basin and datasets}

The target basin of the Senegal River, shared by Guinea, Mali, Senegal, and Mauritania, presents a strong decreasing gradient of rainfall in northerly direction (Fig. 1). The Organization for the Development of the Senegal River (Organisation pour la mise en valeur du fleuve Sénégal, OMVS), is the river basin organization in charge of the management and planning of water resources in the region

With less than $200 \mathrm{~mm} \mathrm{yr}^{-1}$ in the north (Sahel), to more than $2000 \mathrm{~mm} \mathrm{yr}^{-1}$ in the south (headbasin), the rainfall seasonal cycle is unimodal (Sandholt et al., 2003). The basin corresponds to three eco-regions, Sahelian savanna (north), West Sudanian savanna, and Guinean forest savanna (south).

Observed daily rainfall grids compiled by the IRD (Institut de Recherche pour le Developpement; previously ORSTOM, France), with spatial resolution of $1^{\circ}$, have been used. These grids were previously considered in other studies in West Africa (Diedhiou et al., 1999; Janicot and Sultan, 2001; Messager et al., 2004; García Galiano and Giraldo Osorio, 2010; Karambiri et al., 2011). Six RCMs were selected, driven by different GCMs (Global Climate Models) for scenario A1B: GKSS/CLM (ECHAM5), METO-HC/HAD (HadCM3Q0), KNMI/RACMO (ECHAM5-r3), INM/RCA (HadCM3Q0), SMHI/RCA (HadCM3Q0), MPI/REMO 
(a)

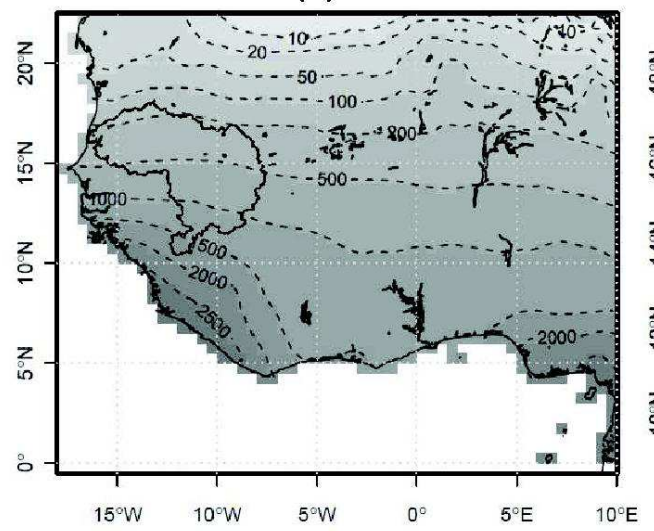

(b)

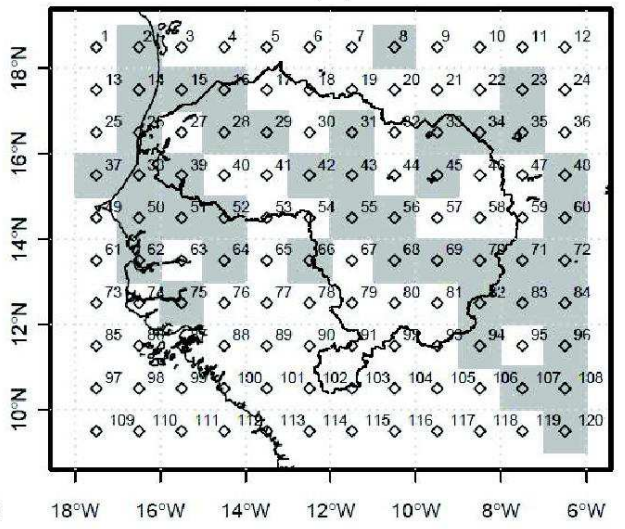

Fig. 1. Location of Senegal River Basin: (a) mean annual rainfall (mm) from Climate Research Unit database (CRU, University of East Anglia, Norwich, UK), over 1961-1990 period, and (b) sites selected for analyses, and The IRD sites are highlighted with gray squares.

(ECHAM5-r3), provided by the European ENSEMBLES Project (Christensen et al., 2009). The selection of RCM was based on its temporal coincidence with the IRD data (period 1970-1990), which enables the bias analysis. For the analysis, 120 sites on a regular grid of $1^{\circ} \times 1^{\circ}$ were selected. However, the spatial discontinuity of the IRD data constrains the bias analysis only on 43 of these sites (Fig. 1). The AMDR series were obtained from the observed and simulated daily rainfall grids, for the sites defined.

\section{Methodology}

A critical need in the research of climate change impacts focuses on quantifying the uncertainty associated with future climate projections. The results of impacts studies on hydrometeorological extremes and runoff in the study region, have shown a considerable divergence (García Galiano an Giraldo Osorio, 2010; Karambiri et al., 2011), which increases the uncertainty in predicting climate change at regional level using RCMs (Giorgi and Mearns, 2002). Several authors have contrasted results from RCMs to presentday data, trying to explain the dynamics of WAM, and interaction with precipitation. Among them, Sylla et al. (2009) worked with precipitation and temperature from ICTPRegCM3 RCM nested in both reanalysis data and ECHAM5 GCM simulations, and observed data from Climate Research Unit database (CRU, University of East Anglia, Norwich, UK); and Sylla et al. (2010b) analyzed intraseasonal and interannual variability of rainfall taking into account the same RCM driven by reanalysis data. The performance of the RCM, considering several observed datasets, was eval- uated by Sylla et al. (2010a) for seasonal mean climatologies, annual cycle and interannual variability over Africa. They found biases from the regional model as well as those brought through the boundary forcing, and have concluded that a larger set of RCMs and GCMs data comparisons could be better for general applicability.

Since it is not possible to conclude which model seems to be the most reliable, a comprehensive assessment of climate change projections needs to be based on the information provided by the RCM ensemble approach to simulate the variability of the rainfall, according to Giorgi and Mearns (2002). Based on these premises, the GAMLSS (Generalized Additive Models for Location, Scale and Shape) tool, proposed by Rigby and Stasinopoulos (2005), is applied to simulate the nonstationarity of the PDF of maximum rainfall in each site. The GAMLSS approach corresponds to semi-parametric regression models (Rigby and Stasinopoulos, 2005; Stasinopoulos and Rigby, 2007). In the present work, four theoretical probability distributions of two parameters were considered: Gumbel (GU), Gamma (GA), Lognormal (LN), and Weibull (WEI). Several previous studies have applied GAMLSS for modeling nonstationary extreme hydrometeorological time series (Villarini et al., 2009; Karambiri et al., 2011), considering similar PDFs.

In the next section, the methodology for weighting the RCMs using the REA factors (Giorgi and Mearns, 2002), and the score proposed by Perkins et al. (2007) considered for assessing PDFs agreements, are introduced. Finally, the PDF ensemble methodology is explained.

Hydrol. Earth Syst. Sci., 15, 3605-3615, 2011 
3.1 Reliability Ensemble Averaging (REA) method and Perkins score

The REA method provides a measure of reliability, average and uncertainty range of simulated climate change from ensembles of different atmosphere-ocean general circulation models and RCMs (Giorgi and Mearns, 2002). Previous studies have used the REA method to evaluate the performance of climate models (Giorgi and Mearns, 2003; Bark et al., 2010; Dominguez et al., 2010; Mote and Salathé, 2010; Räisänen et al., 2010)

The method considers two criteria to estimate the model reliability factor $-R$ in Eq. (1). The first of them, model performance criterion $\left(R_{\mathrm{B}}\right)$, considers the performance of the model in reproducing present-day climate. The second one, model convergence criterion $\left(R_{\mathrm{D}}\right)$, evaluates the convergence to the "best estimated response" or REA average in the future climate projections. For reaching high reliability for a given RCM, both criteria should be met in Eq. (1). Giorgi and Mearns (2002) define the reliability factor for model $i$ as follows:

$R_{i}=\left[\left(R_{\mathrm{B}, i}\right)^{m} \times\left(R_{\mathrm{D}, i}\right)^{n}\right]^{[1 /(m \times n)]}$

where the parameters $m$ and $n$ are criterion weights. In this work, we assume $m=n=1$, giving equal weight to both criteria.

For estimation of the $R_{\mathrm{B}}$ and $R_{\mathrm{D}}$ parameters (Eq. 1), the Perkins score method (Perkins et al., 2007) was used. The Perkins score is a simple quantitative measure of the degree of agreement between the PDFs obtained from the RCMs and that observed from the data. This metric measures the common area between the two curves of the PDFs.

The Perkins score has been applied by several authors to assign weights to different climate models of an ensemble, for the analysis of several hydrometeorological variables (Perkins and Pitman, 2009; Boberg et al., 2009, 2010; Smith and Chandler, 2010).

In the case of the estimation of model performance criterion $R_{\mathrm{B}}$, the cumulative distribution functions (CDFs) were built from observed data and from RCMs over the 19701990 time period. For the assignment of a cumulative probability to each value in the ordered series, the inverse of the Weibull equation was used.

For the model convergence criterion $R_{\mathrm{D}}$, the difficulty is that there is no AMDR PDF known for furture climate. According to Giorgi and Mearns (2002), an iterative process is followed to obtain the estimated PDF and therefore to estimate $R_{\mathrm{D}}$. The estimated PDF is built using bootstraping techniques with $N=1000$ data, considering the simulated series for the models between 2021-2050 (30 yr). Initially, the reference PDF is built by assigning equal skill scores to all RCMs (this is, each model consists of $1000 / 6 \approx 167$ data, obtained from sampling with replacement from the simulated series of $30 \mathrm{yr}$ ). Then, the distance of each RCM to the estimated PDF is calculated and consequently the assigned

Hydrol. Earth Syst. Sci., 15, 3605-3615, 2011 weights are readjusted. This procedure converges quickly after some iterations. It should be noted that the PDF built in this way is only an estimate of the distribution of the AMDR of future climate projection. The REA average does not represent the actual climate response to the climate forcing scenarios, however the REA average represents the best estimate of this response (Giorgi and Mearns, 2002, 2003).

The values of $R_{\mathrm{B}}, R_{\mathrm{D}}$ and $R$ are estimated in the sites where the IRD is available (Fig. 1). Then, these values are interpolated (using the method of inverse distance), and the spatial distributions of the reliability factors (Fig. 2) are obtained for the selected RCMs.

According to Giorgi and Mearns $(2002,2003)$, the likelihood associated with a simulated change for the $\mathrm{RCM}_{i}$ is proportional to the model reliability factor $R_{i}$. In this, the results of models with higher reliability factor are more likely to occur. The normalized reliability factors, $P m$ in Eq. (2), can be interpreted as this likelihood associated with each RCM. Several authors (Tebaldi et al., 2005; Tebaldi and Knutti, 2007), have shown that the normalized reliability factors are analogous to the accuracy factors defined in their ensembles built by Bayesian approaches. The likelihood $P m$ for each RCM is defined as follows (Giorgi and Mearns, 2003):<smiles>CC(C)(C)[V]</smiles>

Maps algebra was used to build the $P m$ raster, using the interpolated maps of $R$. The $P m$ maps (Fig. 2) allowed building the PDF ensembles in sites where the IRD data was not available, e.g. the south part of the domain.

\subsection{Calculation of PDF ensemble}

The GAMLSS adjustment to various series of AMDR in each site predicts trends that differ in magnitude and sign, depending on the RCM and site considered. As an example, the temporal variation of the AMDR PDF is represented with curves for different percentiles $(5,10,25,50,75,90$, and $95 \%$ ), applying GAMLSS to the six RCMs selected for site 16 (Fig. 3). The goodness of fit to the statistical model was assessed, considering the normality of the residuals, visual inspections of the qq-plots and the worm plot (not shown), according to the methodology presented by van Buuren and Fredriks (2001)

Therefore, for the building of the PDF ensemble in each site, greater weight was given to RCMs with high value of normalized reliability factor, using bootstrapping techniques (Efron and Tibshirani, 1993). Once the $P m$ maps were obtained, the PDF ensembles are built on each site of the study region. Random subsamples of size $N=10000$ were built, considering the RCM in proportional fashion to its normalized reliability factor (e.g. on site 16,1550 values from GKSS/CLM, 1330 from METO-HC/HAD, and so on). To obtain the evolution of the probability distribution of AMDR

www.hydrol-earth-syst-sci.net/15/3605/2011/ 


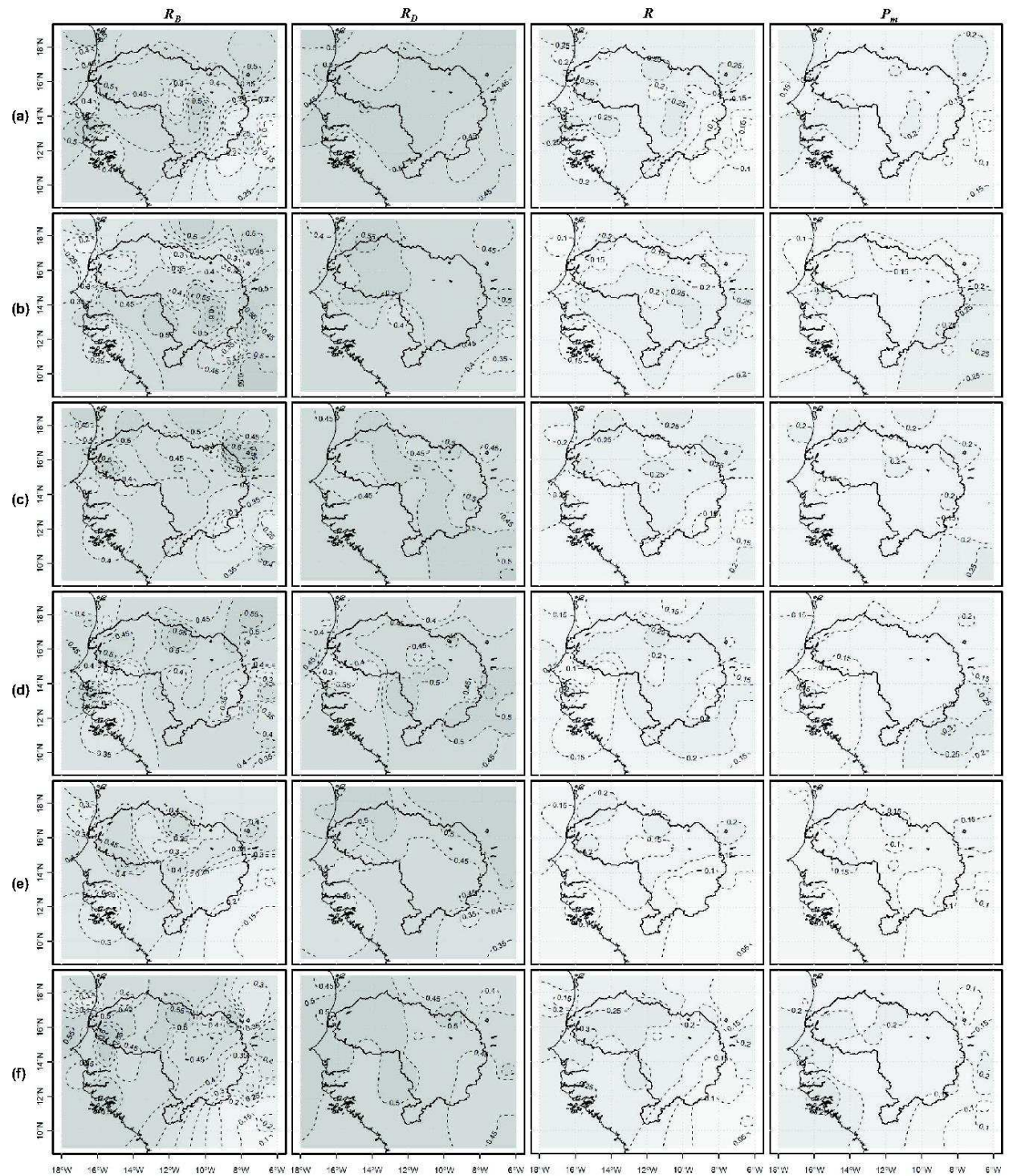

Fig. 2. Maps of RCM reliability factors $R_{\mathrm{B}}, R_{\mathrm{D}}, R$ and $P m$ : (a) GKSS/CLM, (b) INM/RCA, (c) KNMI/RACMO, (d) METNO-HC/HAD, (e) MPI/REMO, and (f) SMHI/RCA 

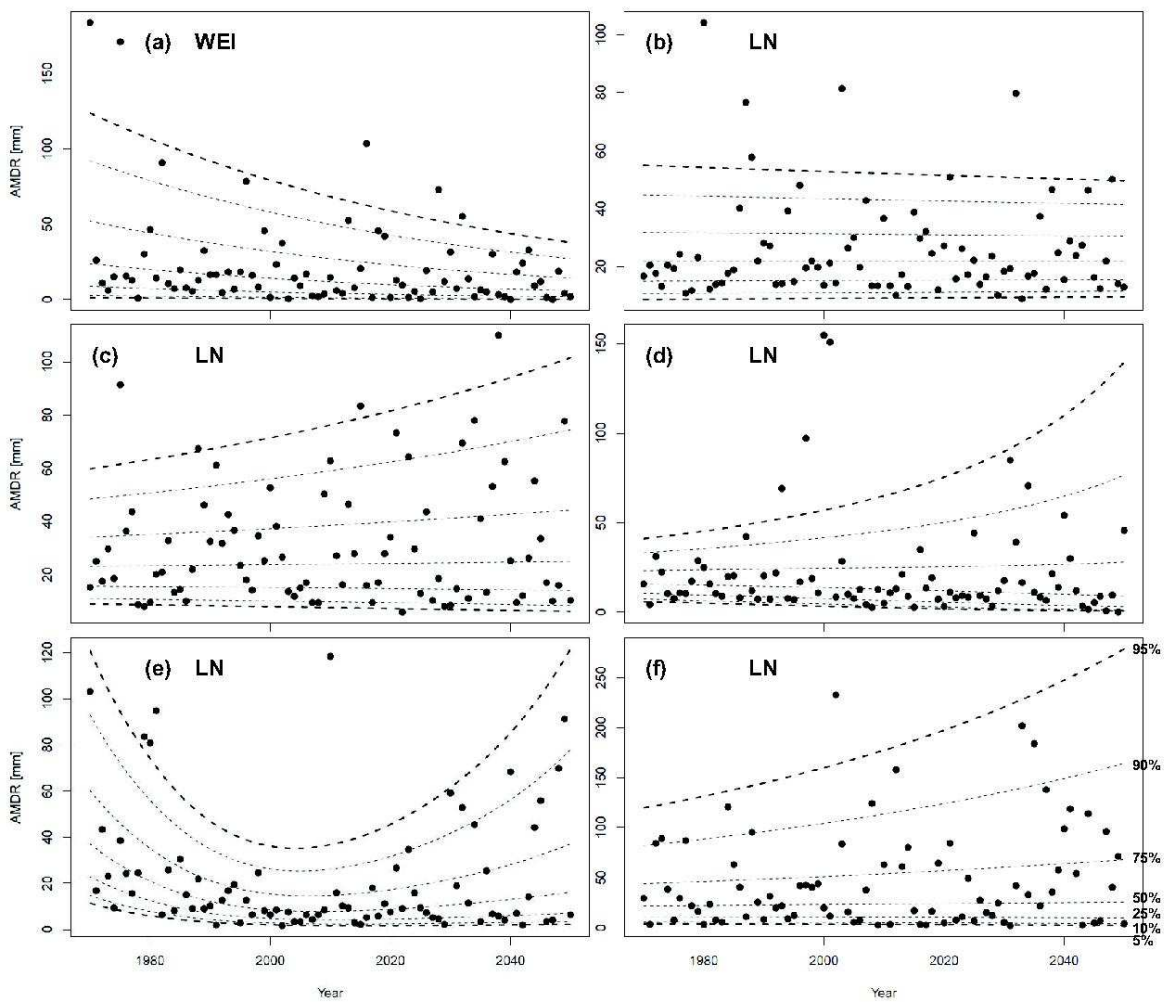

Fig. 3. GAMLSS analysis of AMDR (mm) for site 16 for several RCM: (a) GKSS/CLM, (b) METO-HC/HAD, (c) KNMI-RACMO, (d) INM-RCA, (e) SMHI/RCA, and (f) MPI-MREMO. The centile curves ( 5 to $95 \%$ ) are represented by dashed lines. It should be noted that the ordinate scale is automatically fixed.

in each site, PDF ensembles were constructed for each ten years in the period 1970-2050, and basic statistics (mean $\mu$ and standard deviation $\sigma$ ) and the quantiles for $5,10,25,50$, 75,90 , and $95 \%$ with their respective confidence intervals $(95 \% \mathrm{CI})$ were estimated.

From Fig. 4 , for PDF ensemble in site 16, the difference in magnitude and sign of GAMLSS fit becomes more evident (e.g. the adjusted trend for the SMHI/RCA and MPI/REMO AMDR series indicates a plausible increasing, but with mean values of AMDR differences of about $30 \mathrm{~mm}$, while the GKSS/CLM presents a negative trend). Another feature of adjustment is that despite having the lowest value of the model performance criterion $R_{\mathrm{B}}$, the INM/RCA model presents the highest value of the model convergence criterion $R_{\mathrm{D}}$. Although this situation is not presented in all the sites analyzed, Giorgi and Mearns (2002) claimed that a large individual model bias does not imply a large model divergence.

Hydrol. Earth Syst. Sei., 15, 3605-3615, 2011

\section{Results: maps of AMDR PDF statisties}

Once the statistics for the PDF ensemble at each site have been obtained for the period 1970-2050, these values were interpolated to generate maps depicting the spatio-temporal evolution of the AMDR distribution. In Fig. 5, the maps constructed for the mean, standard deviation, and for $90 \%$ and $95 \%$ quantiles $(T r=10 \mathrm{yr}$ and $T r=20 \mathrm{yr}$, respectively) for 1990 and 2050, and their percentage difference, are presented. Using the Monte Carlo method based on bootstrapping with replacement (Efron and Tibshirani, 1993; Sánchez et al., 2009), applied to AMDR populations obtained from PDF ensemble, the sites where these differences were statistically significant for $95 \%$ bootstrap confidence intervals have been shown on difference maps with a dark grey square (Fig. 5). 


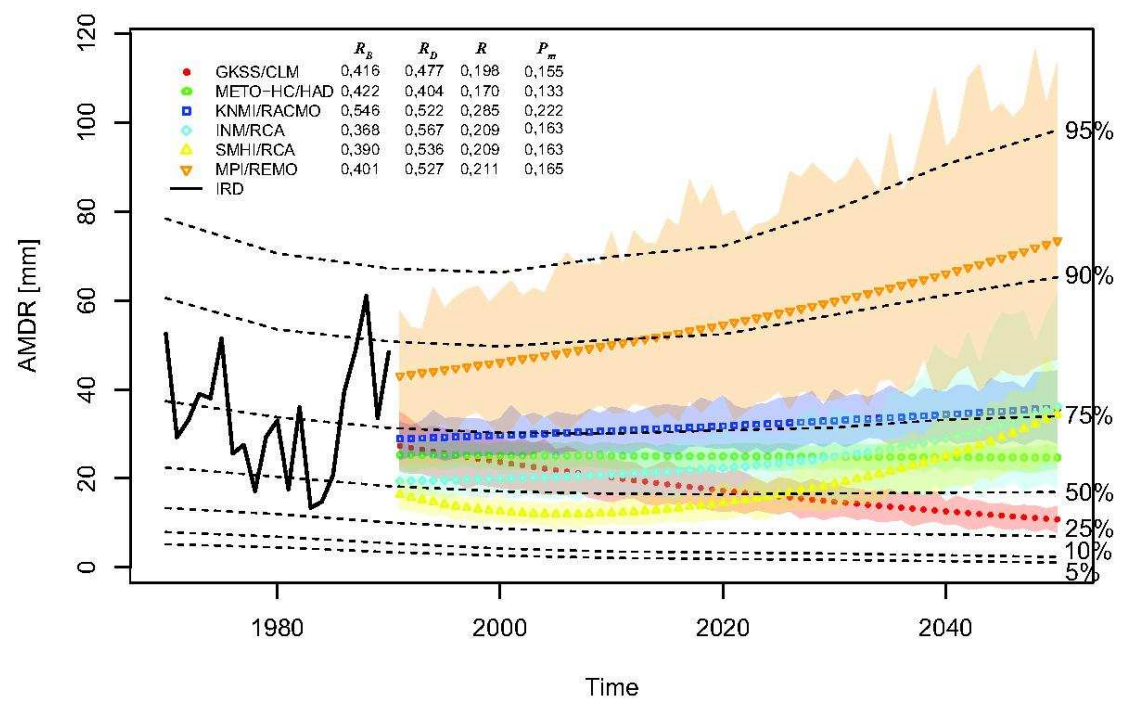

Fig. 4. PDF ensemble on site 16. The dashed lines show 5 to $95 \%$ quantiles of PDF ensemble, and markers represent the RCM mean values obtained from the fitted PDF. The polygons show the mean variability in the period 1991-2050, using the $95 \%$ CI computed with bootstrapping. The IRD AMDR series in 1970-1990 is presented as a solid line. The computed REA values $\left(R_{\mathrm{B}}, R_{\mathrm{D}}, R\right.$ and $\left.P m\right)$ used to build the PDF ensemble, are presented.

The maps constructed for $\mu$ (Fig. 5a) have a spatial structure that matches the latitudinal gradient of mean annual precipitation in the region (shown in Fig. 1), nonetheless the dif ference between them does not preserve this structure. Significant increase in $\mu$ is expected for 2050 in the Senegal River Valley and the upper basin, while in the Sahelian zone a decrease is projected, but it is not significant. In the Valley area, the expected increase in $\mu$ will be about $10 \mathrm{~mm}$, very similar to the increment for the upper basin, yet in the lower basin this represents a significant increase of over $30 \%$. The maps of $\sigma$ (Fig. 5b) retain the latitudinal gradient of rainfall maps, although less significantly than the $\mu$ maps. The difference between the two maps shows that in the lower basin a significant increase in $\sigma$ is projected for 2050 , which could reach $80 \%(25 \mathrm{~mm})$, while in the upper basin the projected increase in this parameter hardly exceeds $25 \%(10 \mathrm{~mm})$. Finally, the maps for $T r=10$ and $T r=20 \mathrm{yr}$ (Fig. $5 \mathrm{c}$ and $\mathrm{d}$ respectively) show a clear latitudinal gradient, with a northward decrease in AMDR. A general increase in the AMDR associated with both return periods is expected for 2050 , ex cept at the northern edge of the basin and in some isolated areas of the study area within the Sudanian zone. With regard to $T r=10 \mathrm{yr}$, a significant increase is foreseen in the lower basin, where a difference of more than $20-25 \mathrm{~mm}$ was calculated between the reference years $(30-40 \%)$. On the other hand, a significant greater increase associated with $T r=20 \mathrm{yr}$ is estimated for the same region, where the difference is about $30-35 \mathrm{~mm}(40-50 \%)$

Previous studies in the area with similar objectives, have been developed. The maps obtained by Garcia Galiano and Giraldo Osorio (2011) exhibit spatial trends very similar to those presented in the Fig. 5, however, they are numerically quite different. These authors estimated increases of about $40 \%$ for $\mu$, and up to $100 \%$ for $\sigma$, higher than calculated values in the present work, with more areas appearing with significant differences in their maps.

\section{Discussion and conclusions}

In order to distinguish the predicted changes in the severity and frequency of AMDR in the Senegal River Basin, plausible climate scenarios provided by RCMs and observed data were considered. The GAMLSS methodology and ensemble techniques allow to simulate the non-stationarity present in AMDR series for the study region.

Nevertheless, the good fit of the GAMLSS statistical model to simulated AMDR time series neither implies a proper coupling to the observed series from the IRD database, nor a proper convergence between the PDFs of AMDR future projections. In order to exploit all the information provided by the RCMs, a PDF ensemble is constructed

Hydrol. Earth Syst. Sci., 15, 3605-3615, 2011 


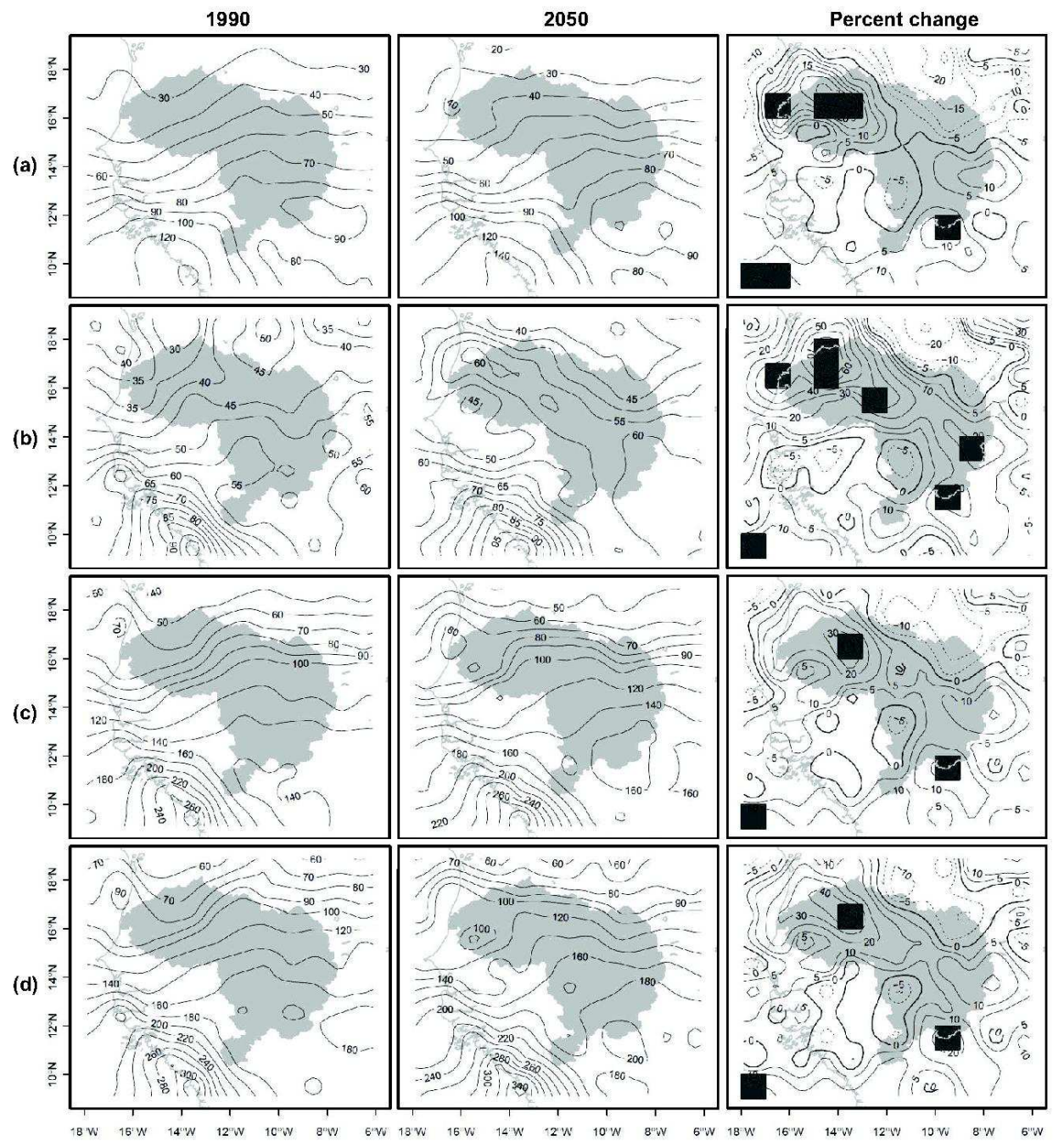

Fig. 5. Interpolated maps from the GAMLSS analysis for each site: (a) mean $\sigma$, (b) standard deviation $\sigma$; (c) associated with $T r=10 \mathrm{vr}$, and (d) $T r=20$ yr. The maps for the years 1990 and 2050 , and the percentage difference between them, are presented. In the difference maps, negative values are dashed, while the $95 \%$ confidence in projected changes is highlighted with the dark gray square.

for each site in the region based on the bias analysis, allowing the estimation of the spatio-temporal change of AMDR in the study area.

The use of weighted ensembles is justified by the fact that there are clear differences between the performance of the RCMs to simulate the present-day AMDR, where the best

Hydrol. Earth Syst. Sei., 15, 3605-3615, 2011 model may have nearly twice the skill score with respect to the worst model in some sites. However, the use of different weights affects the significance of the results. Several studies have addressed this issue, with the aim of designing more sophisticated methods for combining models. However, to know whether uncertainty in the evolution of future

www.hydrol-earth-syst-sci.net/15/3605/2011/ 
climate will remain at a similar level or whether it will be reduced substantially in the next decades, remains a challenge (Tebaldi and Knutti, 2007).

As key findings, from the interpolated maps various parameters of the PDF ensemble maintain the latitudinal gradient of rainfall in the area. However, in the difference maps for the reference years considered ( 1990 and 2050 ), this spatial pattern is not preserved. The percentage difference shows that in the Valley area, the increase of AMDR will be significantly higher than in the upper watershed area. In the Valley area, the increase in $\mu$ is projected to be more than $30 \%$ and the increase in $\sigma$ to be close to $80 \%$. Both increases will be reflected in the maximum precipitation amount for the $90 \%$ quantile, which will rise between $30-40 \%$ in the lower part of the basin, and for the $95 \%$ quantile, with a 40 $50 \%$ increase.

Future research lines will be to highlight the deeper analysis of robust and reproducible methods of estimation of skill scores associated with each RCM in the ensemble. With the aim of its application on spatio-temporal plausible patterns of other daily rainfall features (maximum dry spel lengths, annual or seasonal number of days without rainfall, etc.), and hydrometeorological variables (evaporation, temperatures, monthly rainfall) with implications in water resources management at basin scale. Even considering their application in regions with different hydroclimatic characteristics (such as the Mediterranean area). The ensemble procedure applied in this study should be compared with other proposals for calculating the weighting, in order to verify the spatial-temporal consistency of the methodological approach.

Another issue to take into account is whether the improvement in resolution of the models will affect the conclusion of the studies. Wehner et al. (2010) found that at high resolutions, the climatic models can produce precipitation values of comparable magnitude to high quality observations. However, at the resolutions typical of the coupled GCM, the precipitation is underestimated.

As it was shown, the future climate change will affect the frequency and intensity of natural hazards, such as extreme rain events, on Senegal River Basin. The impact on society depends on many factors, including the sequence and intensity of events, but also depends on the ability of the people to adapt and recover from the natural hazard. The knowledge and awareness about climate change by stakeholders is important.

In conclusion, a novel PDF ensemble approach was presented, which allows the identification of plausible spatial trends from continuously changing AMDR frequency distributions. The key findings of this study could be considered by OMVS, for designing and implementing effective adaptation strategies to climate variability and change in the region.

www.hydrol-earth-syst-sci.net/15/3605/2011/
Supplementary material related to this article is available online at: http://www.hydrol-earth-syst-sci.net/15/3605/2011/ hess-15-3605-2011-supplement.pdf.

Acknowledgements. The support of the AMMA project is gratefully acknowledged. Based on a French initiative, AMMA was built by an international scientific group and is currently funded by a large number of agencies, especially from France, UK, US and Africa. It has been the beneficiary of a major financial contribution from the European Community's Sixth Framework Research Programme. Detailed information on scientific coordination and funding is available on the AMMA International web site http://www.amma-international org. The authors acknowledge to IRD for providing observed daily rainfall.

Edited by: C. de Michele

\section{References}

Bark, R. H., Colby, B. G., and Dominguez, F.: Snow days? Snowmaking adaptation and the future of low latitude, high elevation skiing in Arizona, USA, Climatic Change, 102, 467-491, doi:10.1007/s10584-009-9708-x, 2010.

Boberg, F., Berg, P., Thejll, P., Gutowski, W. J., and Christensen, J. H.: Improved confidence in climate change projections of precipitation evaluated using daily statistics from the PRUDENCE ensemble, Clim. Dynam., 32, 1097-1106, 2009.

Boberg, F., Berg, P., Thejll, P., Gutowski, W. J., and Christensen, J. H.: Improved confidence in climate change projections of precipitation further evaluated using daily statistics from ENSEMBLES models, Clim. Dynam., 35, 1509-1520, 2010.

Buser, C. M., Künsch, H. R., Lüthi, D., Wild, M., and Schär C.: Bayesian multi-model projection of climate: bias assumptions and interannual variability, Clim. Dynam., 33, 849-868, doi:10.1007/s00382-009-0588-6, 2009.

Christensen, J. H., Rummukainen, M., and Lenderink, G.: Formulation of very-high-resolution regional climate model ensembles for Europe, in: ENSEMBLES: Climate change and its impacts at seasonal, decadal and centennial timescales: Summary of research and results from the ENSEMBLES project, edited by: van der Linden, P. and Mitchell, J. F. B., Met Office Hadley Centre, FitzRoy Road, Exeter EX1 3PB, UK, 47-58, 2009.

Cour, J. M.: The Sahel in West Africa: countries in transition to a full market economy, Global Environ. Change, 11,31-47, 2001.

Diedhiou, A., Janicot, S., Viltard, A., de Félice, P., and Laurent, H.: Easterly waves regimes and associated convection over West Africa and the tropical Atlantic: Results from the NCEP/NCAR and ECMWF reanalyses, Clim. Dynam., 15, 795-822, 1999.

Dominguez, F. Cañón, J. and Valdes, J.: IPCC-AR4 climate simulations for the Southwestern US: the importance of future ENSO projections, Climatic Change, 99, 499-514, doi: $10.1007 / \mathrm{s} 10584$ 009-9672-5, 2010

Efron, B. and Tibshirani, R. J.: An introduction to the bootstrap, Chapman \& Hall, New York, NY, USA, 1993.

Fontaine, B., Roucou, P., and Monerie, P. A.: Changes in the African monsoon region at medium-term time horizon using 12

Hydrol. Earth Syst. Sci., 15, 3605-3615, 2011 
AR4 coupled models under the Alb emissions scenario, Atmos. Sci. Lett., 12, 83-88, doi:10.1002/asl.321, 2011.

García Galiano, S. G. and Giraldo Osorio, J. D.: Analysis of impacts on hydrometeorological extremes in the Senegal River Basin from REMO RCM, Meteorol. Z., 19, 375-384, 2010.

García Galiano, S. G. and Giraldo Osorio, J. D.: Non-stationary analysis of spatial patterns of extreme rainfall events in West Africa, in: Hydro-climatology: Variability and Change (Proceedings of IAHS Lead Symposia held during IUGG2011 in Melbourne, Australia, July 2011), IAHS Publ. 344, IAHS Press, CEH Wallingford, Oxfordshire, United Kingdom, 75-81, 2011

Giorgi, F.: A Simple Equation for Regional Climate Change and Associated Uncertainty, J. Climate, 21, 1589-1604, doi: $10.1175 / 2007$ JCLI1763.1, 2008.

Giorgi, F. and Mearns, L. O.: Calculation of average, uncertainty range, and reliability of regional climate changes from AOGCM simulations via the "reliability ensemble averaging" (REA) method, J. Climate, 15, 1141-1158, 2002.

Giorgi, F. and Mearns, L. O.: Probability of regional climate change based on reliability ensemble averaging (REA) method, Geophys. Res. Lett., 30, 311-314, doi:10.1029/2003GL017130, 2003.

Huntington, T. G.: Evidence for intensification of the global water cycle: review and synthesis, J. Hydrol., 319, 83-95, 2006.

Janicot, S. and Sultan, B.: Intra-seasonal modulation of convection in the West African monsoon, Geophys. Res. Lett., 28, 523-526, 2001.

Karambiri, H., García, S. G., Giraldo, J. D., Yacouba, H., Ibrahim, B., Barbier, B., and Polcher, J.: Assessing the impact of climate variability and climate change on runoff in West Africa: the case of Senegal and Nakambe River basins, Atmos. Sci. Let., 12, 109 115, doi: $10.1002 /$ as1.317, 2011

Kharin, V. V. and Zwiers, F. W.: Estimating Extremes in Transient Climate Change Simulations, J. Climate, 18, 1156-1173, 2005.

Kharin, V. V., Zwiers, F. W. Zhang, X., and Hegerl, G. C.: Changes in Temperature and Precipitation Extremes in the IPCC Ensemble of Global Coupled Model Simulations, J. Climate, 20, 14191444, doi:10.1175/JCLI4066.1, 2009.

Kyselý, J. and Beranová, R.: Climate-change effects on extreme precipitation in central Europe: uncertainties of scenarios based on regional climate models, Theor. Appl. Climatol., 95, 361-374, doi:10.1007/s00704-008-0014-8, 2009

Kundzewicz, Z. W., Mata, L. J., Arnell, N. W., Döll, P., Kabat, P., Jiménez, B., Miller, K. A., Oki, T., Sen, Z., and Shiklomanov, I. A.: Freshwater resources and their management. In Climate Change 2007: Impacts, Adaptation and Vulnerability. Contribution of Working Group II to the Fourth Assessment Report of the Intergovernmental Panel on Climate Change, edited by: Parry, M. L., Canziani, O. F., Palutikof, J. P., van der Linden, P. J. and Hanson, C. E., 173-210, Cambridge University Press, Cambridge, UK, 2007.

Labat, D., Goddéris, Y., Probst, J. L., and Guyot, J. L.: Evidence for global runoff increase related to climate warming, Adv. Water Resour., 27, 631-642, 2004.

Lebel, T., Cappelaere, C., Galle, S., Hanan, N., Kergoat, L., Levis, L., Vieux, B., Descroix, L. Gosset, M., Mougin, E., Peugeot, C. and Seguis, L.: AMMA-CATCH studies in the Sahelian region of West-Africa: an overview, J. Hydrol., 375, 3-13, 2009.

Messager, G., Gallée, H., and Brasseur, O.: Precipitation sensitivity to regional SST in a regional climate simulation during the West African monsoon for two dry years, Clim. Dynam., 22, 249-266, 2004

Milly, P. C. D., Betancourt, J., Falkenmark, M., Hirsch, R. M., Kundzewicz, Z. W., Lettenmaier, D. P., and Stouffer, R. J.: Stationarity is dead: Whither water management?, Science, 319 , $573-574,2008$

Mote, P. W. and Salathé, E. P.: Future climate in the Pacific Northwest, Climatic Change, 102, 29-50, doi:10.1007/s10584-0109848-z, 2010

Nikulin, G., Kjellström, E., Hansson, U., and Strandberg, G.: Evaluation and future projections of temperature, precipitation and wind extremes over Europe in an ensemble of regional climate simulations, Tellus, 63 A, 41-55, doi:10.1111/j.1600$0870.2010 .00466 . x, 2011$.

Paeth, H., Born, K., Girmes, R, Podzun, R., and Jacob, D.: Regional climate change in tropical and northern Africa due to greenhouse forcing and land use changes, J. Climate, 22, 114-132, 2009.

Paeth, H., Hall, N. M. J., Gaertner, M., Alonso, M. D., Moumouni, S., Polcher, J., Ruti, P. M., Fink, A. H., Gosset, M., Lebel, T., Gaye, A. T., Rowell, D. P., Moufouma-Okia, W., Jacob, D., Rockell, B., Giorgi, F., and Rummukainen, M.: Progress in regional downscaling of West African precipitation, Atmos. Sci. Let., 12, 75-82, doi: $10.1002 /$ as1.306, 2011

Perkins, S. E. and Pitman, A. J.: Do weak AR4 models bias projections of future climate changes over Australia?, Climatic Change, 93, 527-558, 2009

Perkins, S. E., Pitman, A. J., Holbrook, N. J., and McAneney, J.: Evaluation of the AR4 climate models' simulated daily maximum temperature, minimum temperature, and precipitation over Australia using probability density functions, J. Climate, 20 $4356-4376,2007$.

Räisänen, J., Ruokolainen, L., and Ylhäisi, J.: Weighting of model results for improving best estimates of climate change, Clim. Dynam. 35, 407-422, doi: 10. 1007/s00382-009-0659-8, 2010.

Ruti, P. M., Williams, J. E., Hourdin, F, Guichard, F., Boone, A., Van Velthoven, P., Favot, F., Musat, I., Rummukainen, M., Domínguez, M., Gaertner, M. Á., Lafore, J. P., Losada, T., Rodriguez de Fonseca, M. B., Polcher, J., Giorgi, F., Xue, Y. Bouarar, I. Law K., Josse, B, Barret B., Yang X and Mari, C.: The West African climate system: a review of the AMMA model inter-comparison initiatives, Atmos. Sci. Let., 12, 116122, doi: $10.1002 /$ asl $305,2011$.

Rigby, R. A. and Stasinopoulos, D. M.: Generalized additive models for location scale and shape, Appl. Stat., 54, 507-554, 2005.

Sánchez, E., Romera, R., Gaertner, M. A., Gallardo, C., and Castro, M.: A weighting proposal for an ensemble of regional climate models over Europe driven by 1961-2000 ERA40 based on monthly precipitation probability density functions, Atmos. Sci. Lett., 10, 241-248, 2009

Sandholt, I., Andersen, J., Dybkjær, G., Nyborg, L., Lô, M., Rasmussen, K., Refsgaard, J. C., and Touré, A.: Integration of earth observation data in distributed hydrological models: the Senegal River basin, Can. J. Remote Sens., 29, 701-710, 2003.

Smith, I. and Chandler, E.: Refining rainfall projections for the Murray Darling Basin of south-east Australia-the effect of sampling model results based on performance, Climatic Change, $102,377-393,2010$.

Stasinopoulos, D. M. and Rigby, R. A.: Generalized additive mod- 
els for location scale and shape (GAMLSS) in: R., J. Stat. Software, $23,1-46,2007$

Sylla, M. B., Gaye, A. T., Pal, J. S., Jenkins, G. S, and Bi X. Q.: High-resolution simulations of West African climate using regional climate model (RegCM3) with different lateral boundary conditions, Theor. Appl. Climatol., 98, 293-314 doi: 10. 1007/s00704-009-01 10-4, 2009

Sylla, M. B., Coppola, E., Mariotti, L., Giorgi, F., Ruti, P. M. Dell'Aquila, A., Bi, X.: Multiyear simulation of the African climate using a regional climate model (RegCM3) with the high resolution ERA-interim reanalysis, Clim. Dynam., 35, 231-247, doi:10.1007/s00382-009-0613-9, 2010a.

Sylla, M. B., Dell'Aquila, A., Ruti, P. M., Giorgi, F.: Simulation of the intraseasonal and the interannual variability of rainfall over West Africa with RegCM3 during the monsoon period, Int. J. Climatol, 30, 1865-1883, doi:10.1002/joc. 2029, 2010b.

Tapiador, F. J., Sánchez, E., and Romera, R.: Exploiting an ensemble of regional climate models to provide robust estimates of projected changes in monthly temperature and precipitation probability distribution functions, Tellus, $61 \mathrm{~A}, 57-71$, doi:10.1111/j.1600-0870.2008.00374.x, 2009.

Tebaldi, C. and Knutti, R.: The use of the multi-model ensemble in probabilistic climate projections, Phil. T. Roy. Soc. A, 365 2053-2075, doi:10.1098/rsta.2007.2076, 2007.
Tebaldi, C, Smith, R. L, Nychka, D., and Mearns, L O. Quantifying Uncertainty in Projections of Regional Climate Change: A Bayesian Approach to the Analysis of Multimodel Ensembles, J. Climate, 18, 1524-1540, 2005.

van Buuren, $S$. and Fredriks, M. Worm plot: a simple diagnostic device for modelling growth reference curves, Statist. Med, 20 $1259-1277,2001$

Villarini, G., Smith, J. A., Serinaldi, F., Bales, J., Bates, P. D., and Krajewski, W. F.: Flood frequency analysis for nonstationary annual peak records in an urban drainage basin, Adv. Water Res, $32,1255-1266,2009$.

Villarini, G., Smith, J. A., and Napolitano, F.: Nonstationary modeling of a long record of rainfall and temperature over Rome, $\mathrm{Adv}$ Water Res, 33, 1256-1267, 2010

Wehner, M. F., Smith, R. L., Bala, G., and Duffy, P.: The effect of horizontal resolution on simulation of very extreme US precipitation events in a global atmosphere model, Clim. Dynam., 34, 241-247, doi:10.1007/s00382-009-0656-y, 2010.

Wigley, T. M. L. and Raper, C. B.: Interpretation of high projections for global-mean warming, Science, 293, 451-454, 2001. 



\subsection{Resumen del Artículo 4}

\subsubsection{Título}

Development of a sub-pixel analysis method applied to dynamic monitoring of floods.

\subsubsection{Objetivos específicos}

- Desarrollar una herramienta de ayuda a la adaptación frente al impacto del cambio y variabilidad climática en las precipitaciones máximas causantes de inundaciones en amplias zonas. Utilizar para ello lenguajes de programación bajo la Licencia Pública General GNU (GNU General Public License, GNU-GPL) en los desarrollos computacionales, para facilitar su aplicación en países en vías de desarrollo (por ejemplo, África Occidental).

- Desarrollar una herramienta de análisis de sub-píxeles (downscaling) para la detección de áreas inundadas, usando imágenes ópticas con moderada resolución espacial desde satélite.

- Verificar que la metodología propuesta mejora los resultados de la clasificación en contraste con los procedimientos tradicionales de clasificación utilizando las mismas imágenes de satélite, en el Valle del Río Senegal (África Occidental). Adicionando para ello, el uso de un DEM.

- Evaluar la bondad de la metodología de clasificación propuesta en diferentes condiciones del terreno (zonas planas y zonas de pendientes pronunciadas).

\subsubsection{Metodología}

Las técnicas de clasificación "duras" de imágenes provenientes de teledetección, asignan a un píxel (celda) grueso una única clase de cobertura. Para las resoluciones más gruesas, los píxeles inevitablemente estarán mezclados, por lo que técnicas de clasificación "suavizadas" pueden ser utilizadas, asignando a un píxel diferentes clases de cobertura (Verhoeye and De Wulf, 2002). El alto número de píxeles mezclados o no clasificados adecuadamente hace necesario obtener información a nivel de sub-píxel, de tal forma que se asegure una estimación precisa del área inundada total (Nyborg and Sandholt, 2001).

El Análisis de Sub-píxeles (SA) pretende aprovechar toda la información contenida en las bandas para cada uno de los píxeles, logrando una clasificación con resolución más fina. La solución que se propone, se apoya en las hipótesis del Modelo de Mezcla Lineal (LMM) en conjunto con hipótesis de dependencia espacial de los datos y un Modelo de Elevación Digital (DEM) de la zona de estudio para la clasificación de las zonas inundadas. El Análisis de Sub-píxeles se ha desarrollado bajo el SIG GRASS 
(Geographical Resources Analysis System). Inicialmente, debe definirse la firma espectral de las clases de cobertura consideradas desde las bandas seleccionadas. El archivo que contiene la firma espectral es la entrada inicial al procedimiento SA (Figura 9). Luego, se aplican los procedimientos LMM y Análisis de Coherencia Espacial (Spatial Coherence Analysis, SCA), empleando el comando desarrollado i.subpixel. Se obtienen así los mapas de fracciones de cobertura para cada clase pura, y su ubicación al interior del píxel grueso. Se promueve le mejor identificación de las zonas inundadas utilizando el DEM subyacente en el SCA. A continuación, se analizan los patrones de drenaje desde el terreno mediante el algoritmo Drenaje basado en DEM (DOD), con el comando i.dod, para mejorar la identificación de zonas inundadas. Finalmente, se aplica el comando i.isolated, que ejecuta un procedimiento de Identificación de Clúster Aislados (ICl), o de pequeños clúster espurios, que se reclasifican para eliminar el ruido del mapa clasificado. Este trabajo, presenta los aspectos teóricos más relevantes de los diferentes procedimientos.

\subsubsection{Resultados}

La zona de estudio corresponde al Valle del Río Senegal. Se utilizó un DEM de la zona de estudio con 3 Arcsec de resolución espacial construida en conjunto por la NASA y la NGA (National Geospatial-Intelligence Agency) en el marco del proyecto SRTM (Shuttle Radar Topography Mission; Farr et al., 2007). Una imagen de reflectancia superficial del sensor MODIS (500 m de resolución espacial, capturada el 12 de enero de 2002), a bordo del satélite TERRA de la NASA, fue utilizada para aplicar la clasificación de SA y otra clasificación supervisada tradicional. Finalmente, la clasificación supervisada de una imagen Landsat 7 ETM+ $(30 \mathrm{~m}$ de resolución espacial), para la misma fecha que la imagen MODIS, fue utilizada como clasificación de referencia para evaluar la bondad de la clasificación propuesta con SA frente a la tradicional.

Los resultados de la clasificación SA fueron evaluados tomando como referencia el mapa clasificado Landsat con resolución espacial modificada a $50 \mathrm{~m}$. La clasificación fue contrastada en varias zonas del valle con inundaciones con diferentes características. En todas ellas la metodología SA mejoró considerablemente la identificación de las áreas inundadas, respecto de la clasificación supervisada. Considerando toda el área de estudio, el método SA identificó correctamente el $80 \%$ de las áreas realmente inundadas, mientras que el método tradicional sólo identificó correctamente el $39 \%$. 


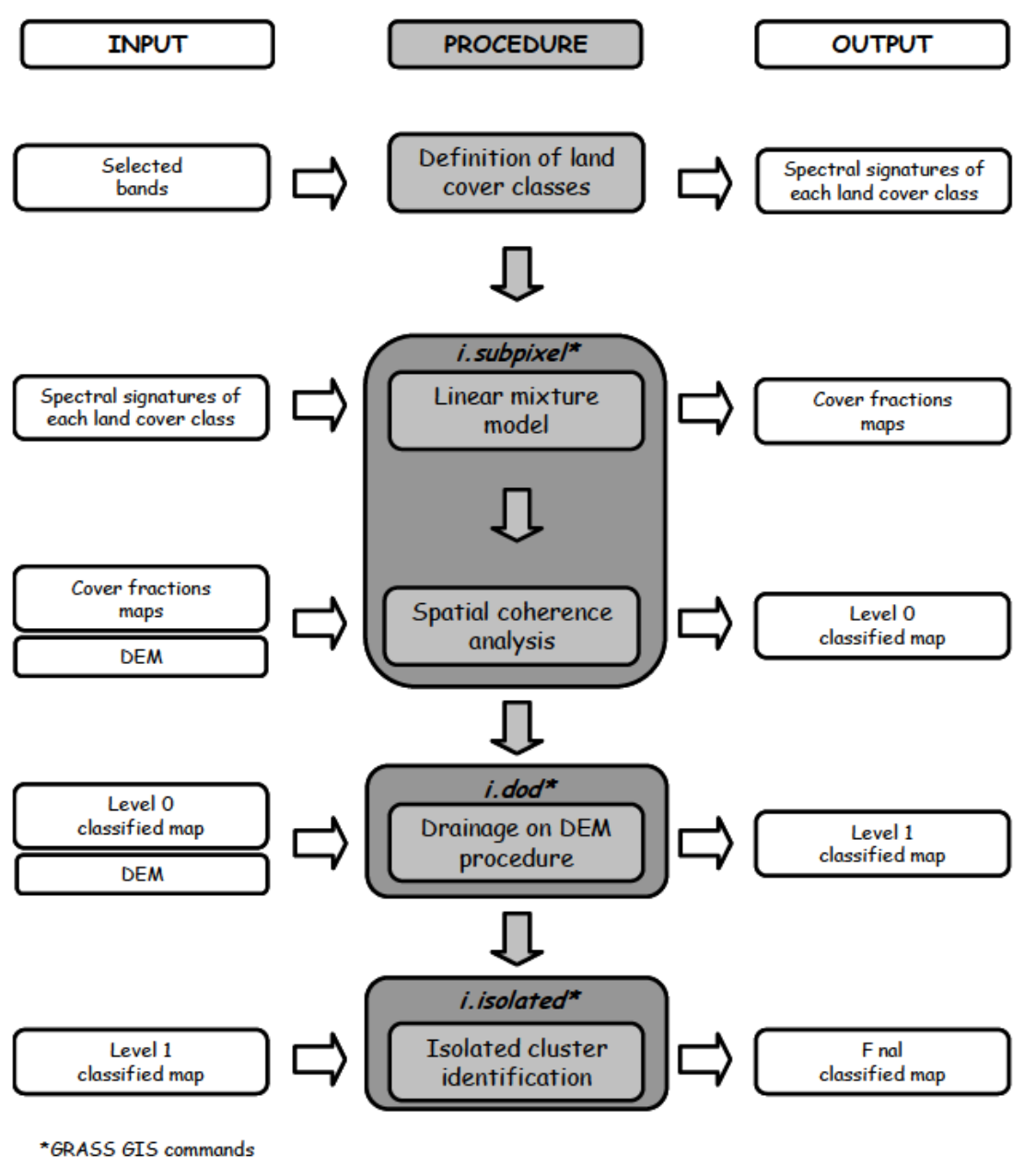

Figura 9. Diagrama de flujo de la metodología de análisis de sub-píxeles desarrollada para la detección de inundaciones. Se destacan los comandos GRASS desarrollados (tomado de Giraldo Osorio and García Galiano, 2012).

\subsubsection{Conclusiones y recomendaciones}

El Análisis de Sub-píxeles ha demostrado ser eficiente en la delimitación de planicies de inundación. El procedimiento desarrollado efectúa una clasificación "suavizada" de mejor resolución espacial, al extraer información a nivel de subpíxel. Se incorporan en el proceso atributos topográficos extraídos desde un DEM de la zona de estudio, para mejorar la identificación de las zonas inundadas.

El Análisis de Sub-píxeles se ha aplicado en la zona del Valle del Río Senegal, mostrando mejores resultados en la clasificación de las zonas inundadas que un algoritmo de clasificación supervisada tradicional.

La metodología desarrollada presenta como ventaja su aplicación directa en el seguimiento de eventos altamente dinámicos, como son las inundaciones, utilizando imágenes de satélite de moderada resolución espacial y con alta resolución temporal. Luego, al basarse en información libremente accesible, su aplicación por parte de responsables de la toma de decisión en países en vías de desarrollo es potenciada. 
Finalmente, debe resaltarse que se contribuye de esta forma a la construcción de "capacidad adaptativa" frente a eventos extremos como las inundaciones, facilitando herramientas que permiten un seguimiento del evento en un tiempo muy cercano al real. 


\title{
5.4.6 Copia de la publicación
}

International Journal of Remote Sensing Vol. 33, No. 7, 10 April 2012, 2277-2295

\section{Development of a sub-pixel analysis method applied to dynamic monitoring of floods}

JUAN DIEGO GIRALDO OSORIO* and SANDRA GABRIELA GARCÍA GALIANO

Department of Civil Engineering, R\&D Group of Water Resources Management, Universidad Politécnica de Cartagena, Paseo Alfonso XIII, 30203, Cartagena, Spain

(Received 23 June 2010; in final form 25 April 2011)

\begin{abstract}
Traditional 'in situ' measurement techniques often fail to record the spatial distribution of floodplains. In that case, remote sensing provides inexpensive and reliable methodologies to map flooded areas and compute flood damage. The identification and monitoring of floods, due to their highly dynamic nature, require the use of high-time-resolution satellite images with the drawback that such images usually have low to medium spatial resolution. In this context, the traditional classification techniques would not be suitable for delineating floods because they use 'hard methods' of classification, where the coarse pixel is assigned to a unique land cover class, generating inaccurate maps of the flooded area. In contrast, the "soft methods' assign several land cover classes within the coarse pixels. In this article, the theoretical basis regarding an innovative methodology of sub-pixel analysis (SA) to identify flooded areas is developed. The improvement in flood delineation is achieved with the use of primary topographic attributes, which stem from a digital elevation model (DEM). The methodology was applied to the monitoring of flood events in the lower Senegal River Valley, using satellite images with moderate spatial resolution. The proposed methodology was demonstrated to be effective for mapping the flood extent: the correct mapping of flooded areas was about $80 \%$ in all considered regions, whilst the better performance of supervised classification was $53 \%$.
\end{abstract}

\section{Introduction}

Floods are highly dynamic events, so monitoring them requires observations with both a large area coverage and a suitable spatiotemporal resolution. An accurate flood spatial mapping will enable us to detect shortcomings in flood control measurements, to organize aid distribution in the most severely affected areas, and once the flood has retreated, to assess the damage claims (Smith 1997).

However, traditional methods for gauging hydrologic variables often fail to record extreme flood events. Furthermore, the remote-sensing techniques have become a reliable and economical method to map and assess flood damage, given the scarcity of gauging networks in developing countries (Sanyal and Lu 2004, Khan et al. 2011).

Within this framework, satellite images have become a key tool for flood monitoring since they provide a synoptic and systematic coverage of the earth's natural phenomena (Zhan et al. 2000, 2002, Tralli et al. 2005, Khan et al. 2011). Together with

*Corresponding author. Email: juan.giraldo@upct.es

Intemational Journal of Remote Sensing
ISSN $0143-1161$ pint'TSSN $1365-5901$ print ISSN 1366-5901 online 02012 Taylor \& Francis

http://www.tandf.co.uk/journals
/dx.doi.org/10.1080/01431161.2011.608091 
geographic information systems (GIS) technology, remote sensing has developed into a key tool to implement regional analysis of flood plains, geomorphologic evolution and land use (Andersen et al. 2001, Sanyal and Lu 2004).

In the monitoring of natural dynamic events such as floods, there is no single remote sensing device suitable for all situations. Optical remote sensors are unable to scan the earth's surface in cloudy conditions (Smith 1997, Brivio et al. 2002, Wang et al. 2002). On the other hand, water surface waves and emerged vegetation increase the roughness, which complicates the delineating of flooded areas with microwave active sensors (Smith 1997, Yang et al. 1999, Wang 2002, Benger 2003, Sanyal and Lu 2004, Martínez and Le Toan 2007). In order to overcome the previous problems, new methodologies have been developed which employ several data sources to detect the flooded areas. The use of images from various platforms and different technologies, together with land use maps and demographic information, allow us to draw up flood risk analysis of human settlements and to assess infrastructure vulnerability (Profeti and Macintosh 1997, Kim and Chi 1998, Wang 2004, Sakamoto et al. 2005, Sanyal and Lu 2005, Töyráä and Pietroniro 2005). The potential of digital elevation models (DEMs), together with satellite images for mapping flooded areas, has been demonstrated by several authors (Giacomelli et al. 1997, Towsend and Walsh 1998, Saich et al. 2001, Brivio et al. 2002, Benger 2003).

Particularly, the monitoring of a flood event needs the use of satellite images with a high time resolution. However, the spatial resolution of these images is not always appropriate, which hampers the correct mapping of flooded areas. Therefore, the development of a methodology of sub-pixel level analysis applied to flooded area mapping is justified. This sub-pixel level analysis must take into account the study zone DEM in the classification process, with the goal of encouraging the mapping of flooded areas in the lower sites of the terrain.

In this article, a sub-pixel analysis (SA) model for the detection of flooded areas using optical images with moderate spatial resolution is proposed. The SA model introduces innovative procedures, considering the study zone DEM in order to improve the detection of flooded areas. The SA model begins with the linear mixture model (LMM) resolution, in order to compute the land cover fractions within the coarse pixels. Afterwards, the spatial dependence of land cover fractions which were identified within the coarse pixel is resolved, using a DEM and supported by two hypotheses: (1) the sub-pixels will tend to be grouped around the land cover class centroid and (2) the 'water' class (or 'flooded' class) sub-pixels will tend to be located in the lower terrain areas.

Subsequently, new flooded zones are mapped using the drainage paths, which are computed from the DEM. In this article, an innovative methodology has been developed in order to incorporate a modified drainage on DEM (DOD) algorithm, which was adjusted to the study's requirements. The outcomes from the original procedure presented by Giacomelli et al. (1997) were sensitive to subjective considerations since it required the user to select a threshold number of flow paths crossing a cell. The modified DOD works with the concept of adding flooded cells in the flow path direction, and is not affected by user subjectivity. Finally, the isolated clusters with few cells are identified and reclassified, using the isolated cluster identification (ICI) procedure modified, based on Jianghong et al. (2004). In contrast with Jianghong et al. (2004), where the isolated clusters could be reclassified only in a single neighbouring land cover class, the new procedure allows us to reclassify the cells in the cluster in 
more than a single land cover, using the neighbourhood land cover as classification criterion.

The results show a great sensitivity of the proposed SA procedure related with the DEM accuracy and its associated topographic maps, which did not hinder a significant improvement of flooded areas identification in the study area.

\section{Background}

The 'hard methods' of classification assign a coarse pixel to a unique land cover class. The pixels will be inevitably mixed in the coarser resolutions, so 'soft methods' could be used in order to assign several land cover classes into the coarse pixel (Verhoeye and De Wulf 2000). The large number of mixed or inadequately classified pixels mean it is necessary to obtain information at the sub-pixel level, in order to guarantee an accurate estimate of the total flooded area (Nyborg and Sandholt 2001). The SA seeks to exploit all the information contained in the bands of the satellite image, achieving a classification with finer resolution in each coarse pixel. The proposed solution is based on LMM hypotheses, together with the hypothesis of spatial dependence and the study zone DEM to perform the flooded areas' classification. In the first place, the LMM and spatial coherence analysis (SCA) procedures are applied: in this way the land cover fraction maps for each end member (or pure class) are obtained, and also its location inside the coarse pixel. Following this, the terrain drainage paths are analysed using the DOD algorithm, to improve the flooded area mapping. Finally, the ICI procedure is applied to identify small spurious clusters with few cells, which are reclassified in order to eliminate the noise in the final classified map. The flow chart of the SA methodology, and the outcomes from the distinct procedures in each stage, is shown in figure 1 . In order to simplify the execution of the SA procedures, several commands in Geographical Resources Analysis System (GRASS) GIS have been developed: first, the isubpixel command runs the LMM and SCA procedures; then the $i . d o d$ command carries out the analysis of the drainage paths with the DOD algorithm; and finally the $i$.isolated command reclassifies the small spurious clusters using the ICI procedure. The main theoretical aspects about the procedures which comprise the SA methodology are explained below.

\section{Linear mixture model}

The LMM supposes that radiance (or reflectance) measured at the sensor is a linear combination of reflected radiances from individual surfaces inside the coarse pixel, whose spectral signatures are unique and well distinguished (Hongen et al. 2004). These individual surfaces are called end members, or pure classes. The analysis is supported on solving a system of simultaneous equations with restrictions. Taking into account all sensor bands, the LMM could be considered as a reasonable abstraction to solve the problem of computing the land cover proportion in a mixed pixel. Therefore, the mathematical model would be (Hu et al. 1999):

$$
\begin{aligned}
& \boldsymbol{R}=\mathbf{E} \cdot \boldsymbol{f}+\boldsymbol{\varepsilon} \\
& \text { such that }\left\{\begin{array}{l}
\boldsymbol{1}^{\mathrm{T}} \cdot \boldsymbol{f}=1 \\
0 \leq \boldsymbol{f}_{\Psi} \leq 1
\end{array},\right.
\end{aligned}
$$

where $\boldsymbol{R}$ is an $n \times 1$ vector which contains the coarse pixel multispectral observation; $\mathrm{E}$ is the $n \times c$ matrix where the element $E_{(\Omega, \Psi)}$ is the spectral response for endmember $\Psi$ in band $\Omega ; f$ is the $c \times 1$ solution vector with land cover fractions, 


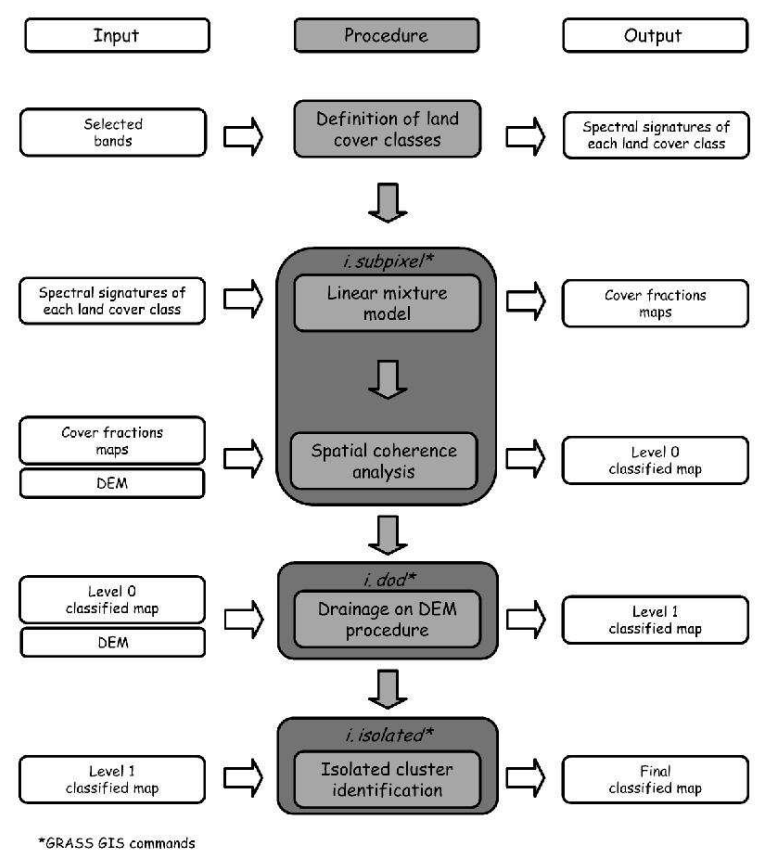

Figure 1. Flow chart of sub-pixel analysis methodology.

$f^{\mathbf{T}}=\left[f_{1}, f_{2}, \ldots, f \Psi, \ldots, f_{c}\right] ; \boldsymbol{\varepsilon}$ is the $n \times 1$ residual error vector; $\mathbf{1}$ is the $c \times 1$ row vector with ones, $\mathbf{1}^{\mathbf{T}}=[1,1, \ldots, 1] ; n$ is the number of used bands; and $c$ is the number of land cover classes.

The minimization of the modulus of the residual error vector $\varepsilon$ gives the values of the land cover fraction $f_{\Psi}$ that best fit each coarse pixel. The first constraint in equation (1) guarantees that the pixel is completely defined by its component endmembers, and can easily be achieved (called the sum-to-one constraint). The second constraint indicates that the contribution of each land cover cannot be negative (positive constraint). In contrast with the sum-to-one constraint, obeying this second constraint is not straightforward. Consequently, the second constraint is generally disregarded when estimating the cover fraction vector $\boldsymbol{f}$, and is considered at a later stage (Klein Gebbinck and Schouten 1995). Solving the proposed problem in equation (1), several authors have concluded that exhaustive searches for a solution give only slightly better results than other simpler methodologies (Klein Gebbinck and Schouten 1995, Kälviäinen et al. 1996, Hu et al. 1999, Lucas et al. 2002). Therefore, the present article proposes to solve the system of equations as an optimization problem. The land cover fraction vector $\boldsymbol{f}$ can be computed if the square sum of the residual error vector $\boldsymbol{\varepsilon}$ is minimized: 


$$
\begin{aligned}
& \min \sum_{\Omega=1}^{n} \varepsilon_{\Omega}^{2}=\sum_{\Omega=1}^{n}\left[R_{\Omega}-R_{\Omega}^{*}\right]^{2}=\sum_{\Omega=1}^{n}\left[R_{\Omega}-\sum_{\Psi=1}^{c} f_{\Psi} E_{(\Omega, \Psi)}\right]^{2} \\
& \text { such that }\left\{\begin{array}{l}
\sum_{\Psi=1}^{c} f_{\Psi}=1 \\
0 \leq f_{\Psi} \leq 1
\end{array},\right.
\end{aligned}
$$

where $\varepsilon_{\Omega}$ is the residual error in band $\Omega ; R_{\Omega}$ is the coarse pixel surface reflectance, received by sensor in band $\Omega ; R_{\Omega}{ }^{*}$ is the coarse pixel surface reflectance, computed with the LMM in band $\Omega ; f_{\Psi}$ is the land cover fraction inside the coarse pixel for endmember $\Psi$; and $E_{(\Omega, \Psi)}$ is the reference value of reflectance for endmember $\Psi$ in band $\Omega$.

As other authors have suggested ( $\mathrm{Hu}$ et al. 1999, Aban et al. 2002), to solve the optimization problem shown in equation (2), the use of Lagrange multipliers is proposed:

$$
L\left(f_{1}, f_{2}, \ldots, f_{c}, \lambda\right)=\sum_{\Omega=1}^{n}\left[R_{\Omega}-R_{\Omega}^{*}\right]^{2}-\lambda\left[\sum_{\Psi=1}^{c} f_{\Psi}-1\right] .
$$

In equation (3), only the sum-to-one constraint is applied. The positive constraint can be applied later, if it is necessary.

When derivatives are carried out and matched to 0 , a system of $c+1$ equations is generated, with $f_{1}, f_{2}, \ldots, f_{c}$ and the Lagrange multiplier $\lambda$ as unknown. Derivatives of the Lagrange equation are as follows:

$$
\begin{aligned}
& \frac{\partial L}{\partial f_{\Psi}}=0=-2 \sum_{\Omega=1}^{n}\left[R_{\Omega}-R_{\Omega}^{*}\right] E_{(\Omega, \Psi)}-\lambda, \Psi=1,2, \ldots, c \\
& \frac{\partial L}{\partial \lambda}=0=1-\sum_{\Psi=1}^{c} f_{\Psi},
\end{aligned}
$$

and the equations system to be solved could be set out as block-matrix format:

$$
\left[\begin{array}{cc}
\mathbf{K}_{(c \times c)} & -\boldsymbol{I}_{(c \times 1)} \\
-\mathbf{1}_{(1 \times c)}^{\mathbf{T}} & 0
\end{array}\right]\left[\begin{array}{c}
\boldsymbol{f}_{(c \times 1)} \\
\lambda
\end{array}\right]=\left[\begin{array}{c}
\boldsymbol{C}_{(c \times \mathbf{1})} \\
-\mathbf{1}
\end{array}\right],
$$

where the elements $K_{j, i}$ and $C_{j}$ have the following expression:

$$
\begin{gathered}
K_{j, i}=2 \sum_{k=1}^{n} E_{(k, j)} E_{(k, i),} \\
C_{j}=2 \sum_{k=1}^{n} R_{k} E_{(k, j)} .
\end{gathered}
$$

The matrix $\mathbf{K}$ is symmetric, hence the lower and upper triangular matrices (LU) decomposition could be used to solve the proposed system. The solved system guarantees compliance with the sum-to-one constraint, but not with the positive constraint (the solution vector may contain $f_{k}<0$ ). One way to solve this problem is by setting the negative proportions to zero and renormalizing the fractions vector (Klein Gebbinck and Schouten 1995). Although the solution obtained is not optimum, this procedure is widely used because it is simple and fast. 
This work aims to find the solution to vector $f$ on the limits of the feasible region. If any $f_{k}<0$ is computed, a new system is built which eliminates the $k$ th row and column in the matrix $\mathbf{K}$, and the $k$ th element in both vectors $\boldsymbol{f}$ and $\boldsymbol{C}$.

$$
\left[\begin{array}{cc}
\mathbf{K}_{(c-1 \times c-1)} & -\mathbf{1}_{(c-1 \times 1)} \\
-\boldsymbol{I}_{(1 \times c-1)}^{\mathrm{T}} & 0
\end{array}\right]\left[\begin{array}{c}
f_{(c-1 \times 1)} \\
\lambda
\end{array}\right]=\left[\begin{array}{c}
\boldsymbol{C}_{(c-1 \times 1)} \\
-1
\end{array}\right] .
$$

The new system is solved, and the solution vector is filled by inserting $f_{k}=0$ in the $k$ th position. The procedure is repeated until $f_{k} \geq 0$. The main advantage of Lagrange multipliers is that they are quick to run, given that the solution is achieved using an analytical process without iterations.

\section{Spatial coherence analysis}

There are several spatial arrangements of each class of sub-pixels which will satisfy the computed land cover fraction values, although only one could maximize the spatial dependence. Consequently, the key problem in the arrangement of the sub-pixels is to define the most likely location of each land cover class within the coarse pixel (Verhoeye and De Wulf 2002). The assumption of spatial dependence, both inside and between the coarse pixels, can resolve this problem.

\subsection{Solution strategy}

Hongen et al. (2004) formulate the SCA as a linear problem, thus:

- the LMM computes the land cover fractions for the $c$ classes, and the coarse pixel is split into $N_{\mathrm{P}}$ sub-pixels $\left(N_{\mathrm{P}}=2^{2}, 3^{2}, 4^{2}\right.$ and so on);

- the number of sub-pixels which must be assigned to class $\Psi$ is $N_{C(\Psi)}$, computed with LMM;

- there is a spatial dependence measurement for each land cover class $\Psi$ and for all sub-pixels $(i, j)$, which is represented by the cost matrix $C_{i, j(\psi)}$; and

- the sub-pixels must be assigned a binary value $(0$ or 1$)$, where 1 indicates the assignment to a particular land cover class, so, the binary variable $X_{i j(\Psi)}$ is defined

$$
X_{i, j(\Psi)}= \begin{cases}1, & \text { if sub }- \text { pixel }(i, j) \text { is assigned to land cover class } \Psi \\ 0, & \text { otherwise. }\end{cases}
$$

The mathematical model is formulated as follows:

$$
\begin{aligned}
& \min \sum_{\Psi=1}^{c} \sum_{j=1}^{\sqrt{N_{\mathrm{P}}}} \sum_{i=1}^{\sqrt{N_{\mathrm{P}}}} C_{i j(\Psi)} X_{i, j(\Psi)} \\
& \text { such that } \\
& \left\{\begin{array}{l}
\sum_{\Psi=1}^{c} X_{i, j(\Psi)}=1, \quad i, j=1,2, \ldots, \sqrt{N_{\mathrm{P}}} \\
\sum_{j=1}^{\sqrt{N_{\mathrm{P}}}} \sqrt{N_{\mathrm{P}}} X_{i=1} X_{i,(\Psi)}=N_{\mathrm{C}(\Psi)}, \quad \Psi=1,2, \ldots, c .
\end{array}\right.
\end{aligned}
$$


The constrains in equation (9) ensure that the sub-pixel $(i, j)$ will be assigned to one, and only one, land cover class, and that exactly $N_{C(\Psi)}$ sub-pixels will be assigned to the class $\Psi$. Both the objective function and the constraints are linear equations, therefore the model could be solved through linear programming techniques (Verhoeye and De Wulf 2002).

\subsection{Cost matrix and DEM}

Some assumptions about the land cover classes arrangement inside the coarse pixel must be included in order to compute the cost matrix $C_{i, j(\Psi)}$ in equation (9). In this work, two main hypotheses have been assumed, which are summarized in figure 2 :

- the class $\Psi$ sub-pixels tend to be grouped around the land cover class centroid; and

- the 'water' class (or 'flooded' class) sub-pixels tend to be located in the lower terrain areas.

In the top pictures in figure 2, the cost matrices in a single coarse pixel can be seen, which has been split into $5 \times 5$ sub-pixels. The bottom pictures show the assignment of 'water' class, according to the cost matrices. The lowest values are represented by dark colours in the cost matrices, whilst the light colours correspond to the highest values.

Figure 2(a) shows the cost matrix assuming only the spatial dependence. The class centroid location has been computed using the land cover fraction from neighbouring coarse pixels inside a kernel window centred in the coarse pixel concerned. The cost will be lower for sub-pixels located close to the centroid. In figure $2(b)$, the cost matrix associated with the underlying DEM of the coarse pixel concerned is presented. The 'flooded' class will be assigned to those sub-pixels in which the DEM indicates the lowest areas. Finally, figure $2(\mathrm{c})$ shows the situation produced by the merging of the two previous criteria for the 'flooded' class (the cost matrix computed from both the SCA procedure and the underlying DEM). The sub-pixel assignation is concordant with both the spatial coherence and the coarse pixel internal topography.

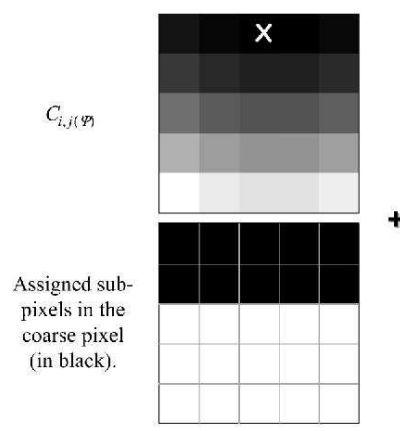

(a)

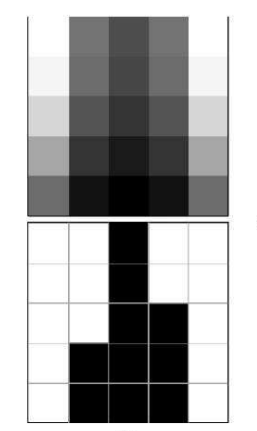

(b)

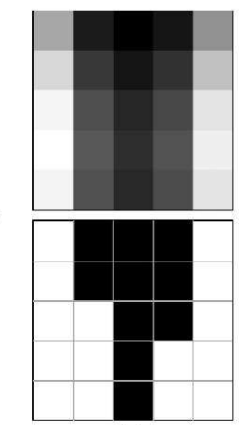

Figure 2. Cost matrix for spatial coherence analysis. (top) and assignment of 'water' class subpixels (bottom). (a) Using the sub-pixel distance to the land cover class centroid (it is marked with ' $\mathrm{X}$ ' in the cost matrix). (b) Using the cost matrix from DEM. It is 'cheaper' to flood the lower areas (in dark grey). (c) Merging the two previous criteria. 
The cost matrix of class $\Psi$, assuming only spatial coherence, is computed using a Gaussian bell as follows:

$$
\begin{aligned}
& C_{i, j(\Psi)}=\frac{1}{2 \pi \sigma_{x(\Psi)} \sigma_{y(\Psi)} \sqrt{1-\rho_{x y(\Psi)}^{2}}} \\
& \left\{1-\exp \left[-\frac{1}{2\left(1-\rho_{x y(\Psi)}^{2}\right)}\left(A^{2}+B^{2}-2 \rho_{x y(\Psi)}^{2} A B\right)\right]\right\} \\
& \text { with } \quad A=\frac{x-x_{\mathrm{c}(\Psi)}}{\sigma_{X(\Psi)}} \quad B=\frac{y-y_{\mathrm{c}(\Psi)}}{\sigma_{y(\Psi)}},
\end{aligned}
$$

where $x_{\mathrm{c}(\Psi)}, y_{\mathrm{c}(\Psi)}$ are the centroid coordinates of land cover class $\Psi ; \sigma_{x(\Psi)}, \sigma_{y(\Psi)}$ is the dispersion around the centroid of land cover class $\psi ; \rho_{x y}(\Psi)$ is the computed covariance of land cover class $\Psi$; and $x, y$ are the coordinates of each sub-pixel, with $(0,0)$ in the coarse pixel central point and being fulfilled $-0.5 \leq x, y \leq 0.5$.

The goal of adding the DEM to re-compute the cost matrix is to improve the mapping of the 'water' sub-pixels. The procedure begins with the assignation of altitude in each sub-pixel $\left(Z_{i, j}\right)$, according to the underlying DEM, and also the maximum and minimum altitude inside the coarse pixel ( $Z_{\mathrm{MAX}}$ and $Z_{\mathrm{MIN}}$, respectively). A vector sorted by ascending order, with $N_{\mathrm{P}}$ elements, is built using the altitude values. The vector element in $N_{\mathrm{C}(\Psi)}$ position, with $\Psi=$ WATER, will define an altitude cut-off value $\left(Z_{\text {CUT }}\right)$. The altitude values inside the coarse pixel are standardized with the following expressions:

$$
\begin{aligned}
& Z_{i, j}^{*}=\frac{Z_{i, j}-Z_{\mathrm{MIN}}+1}{Z_{\mathrm{MAX}}-Z_{\mathrm{MIN}}+2}, \\
& Z_{\mathrm{CUT}}^{*}=\frac{Z_{\mathrm{CUT}}-Z_{\mathrm{MIN}}+1}{Z_{\mathrm{MAX}}-Z_{\mathrm{MIN}}+2} .
\end{aligned}
$$

Subsequently, the factor $F_{i, j(\Psi)}$ is estimated for each sub-pixel and class, with the objective of favouring the location of flooded sub-pixels in the lower areas. This can be expressed in a simple manner as:

- If $\Psi=$ 'water' and: $Z_{i, j}<Z_{\text {CUT }} \Rightarrow F_{i, j(\Psi)}<1$,

$Z_{i, j}>Z_{\text {CUT }} \Rightarrow F_{i, j(\Psi)}>1 ;$

- If $\Psi \neq$ 'water' and: $Z_{i, j}<Z_{\mathrm{CUT}} \Rightarrow F_{i, j(\Psi)}>1$,

$$
Z_{i, j}>Z_{\text {CUT }} \Rightarrow F_{i, j(H)}<1 \text {; }
$$

with the following equation to compute it:

$$
F_{i, j(\Psi)}= \begin{cases}\frac{Z_{i j}^{*}}{Z_{C U T}^{*}}, & \text { if } \Psi=\text { WATER, } \\ \frac{Z_{\mathrm{CUT}}^{\mathrm{UUT}}}{Z_{i_{j}}^{*}}, & \text { otherwise. }\end{cases}
$$

Finally, the cost matrices are re-computed

$$
C_{i, j(\Psi)}=F_{i, j(\psi)} C_{i, j(\psi)},
$$

where matrix $C_{i, j(\Psi)}$ on the right side of the equation is computed using equation (10). 


\section{Drainage on DEM algorithm}

The flooded cells have been placed according to criteria that merge spatial coherence hypotheses and the study zone DEM. Nevertheless, still there will be 'dry' cells which are on the drainage path from the cluster of 'flooded' cells.

Taking in to account the algorithm in Giacomelli et al. (1997), if a 'dry' cell is crossed by a fixed number of drainage paths from 'flooded' cells, it will be reclassified as 'flooded'; it is the user who must decide on the threshold number of flow paths. As the algorithm outcomes are sensitive to the threshold chosen, the present article shows the development of a modified DOD algorithm, in which the outcome is based on aggregation of the flooded cells in the flow path direction. This procedure requires the raster maps of drainage direction and accumulated flow, both of which are associated with the DEM. Figure 3(a) presents a hypothetical situation to explain the algorithm, where the drainage path has been delineated and the flooded cells are shown in grey. The procedure begins with the assumption that a cluster with $n$ 'flooded' cells on the drainage path will reclassify $n-1$ 'non-flooded' cells downstream. It is important to highlight that the 'flooded' clusters are aggregated on the drainage path, not by neighbourhood criteria. Figure 3(b) identifies with ' +1 ', the flooded cells, and with ' -1 ', the dry cells. The aggregation procedure is carried out only in the cells with values greater than 0 . If a '- 1 ' cell upstream has a flooded cluster, it will possibly be reclassified and its value will be added to the accumulative value in the cluster. Figure $3(\mathrm{c})$ explains this procedure: inside the flooded cluster the accumulative value increases, whilst in the dry cells on the cluster drainage path the accumulative value falls until possibly becoming 0 . Finally, the cells in the aggregated map with strictly positive values will be reclassified as 'flooded'. The reclassified map in the example is shown in figure $3(d)$.

\section{Isolated cluster identification}

Isolated regions (or small clusters with a few disconnected cells) are frequently produced throughout the classification process of the satellite images. There are several causes that explain this situation (Jianghong et al. 2004): the classification algorithm lacks sufficient accuracy, some land cover class could have a spectral signature that changes according to natural conditions, the represented area is too small (e.g. a small island in the sea), etc. The sub-pixel level classification produces a huge number of isolated clusters after running the merged procedure LMM-SCA, and so generating a classified map with mistakes that look like the 'salt and pepper' noise of images. With the aim of correcting the classified map, the following methodology is proposed, which is based on the pseudo-code presented by Jianghong et al. (2004).

An isolated cluster is defined as a group of connected cells with a surface area less than a threshold defined by the user. In Jianghong et al. (2004), the isolated clusters were merged with adjacent land cover classes which had the longer shared boundary. The present work proposes to reclassify each cell in the isolated cluster using a Gaussian filter; the Gaussian filter will assign more specific weight to the cells which are closer to the concerned cell to be reclassified. In figure 4 , both the identification and reclassification processes of isolated clusters are shown with an example.

In figure $4(a)$ the raster classified map is shown, which presents some isolated clusters with several surface areas. In figure $4(b)$, the isolated clusters have been identified, taking into account five cells as the threshold surface areas. Finally, figure $4(c)$ shows the reclassified map using the Gaussian filter with a $3 \times 3$ kernel window. It is 

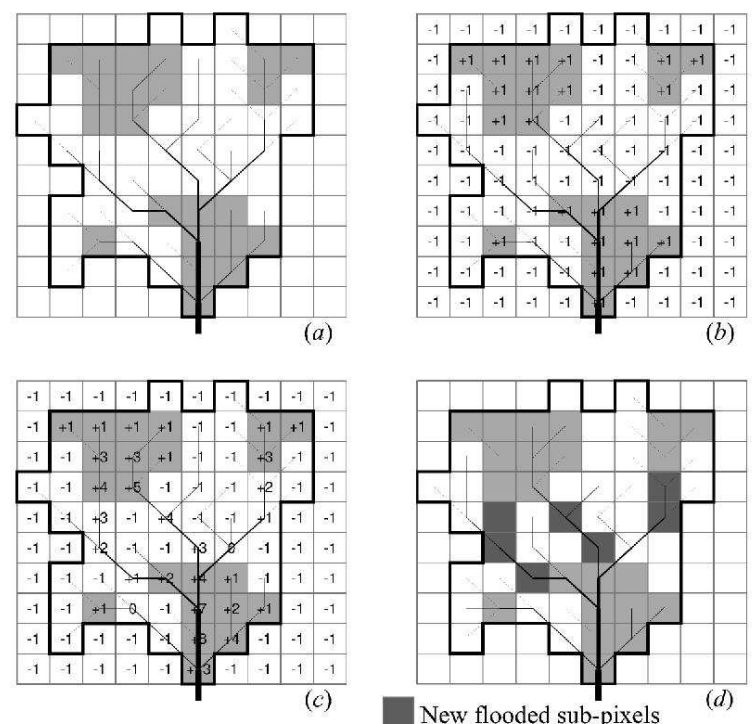

Figure 3. DOD algorithm: ( $a$ ) map of flow directions where the flooded pixels are identified in grey colour, $(b)$ raster map with ' +1 ' in the inundated pixels and " -1 ' in the dry pixels, $(c)$ raster map with the aggregation of inundated clusters in the flow direction and $(d)$ reclassified map with the DOD algorithm.

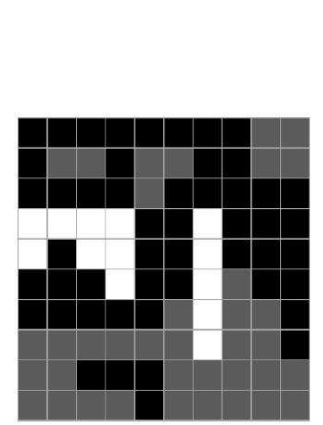

(a)

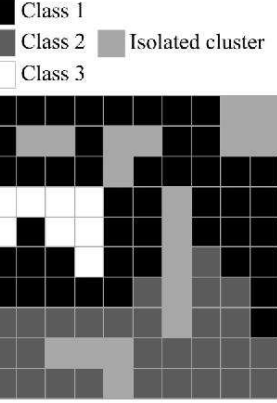

(b)

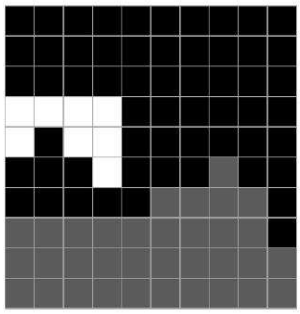

(c)

Figure 4. Isolated cluster identification (ICI) algorithm: (a) raster map with isolated clusters, $(b)$ identification of isolated cluster and $(c)$ reclassified raster map. 
important to highlight the class 3 isolated cluster in the centre, which has been reclassified in both 'black' and 'grey' classes. If the original algorithm proposed by Jianghong et al. (2004) had been used without modifications, then all cells in the cluster would have been reclassified into a single land cover class.

\section{Method validation}

\subsection{Study area and used data}

The study zone is located in the lower Senegal River Valley (between $15^{\circ} 10^{\prime} \mathrm{W}-16^{\circ}$ $45^{\prime} \mathrm{W}$ and $15^{\circ} 30^{\prime} \mathrm{N}-17^{\circ} 5^{\prime} \mathrm{N}$, see figure $5(a)$ ). The detailed regions are presented in figure 5(b): Region 1 corresponds to a wetlands area surrounding the river in Parc de Djoudj, with a flat topography and not well-defined water bodies; while region 2 is the Lac de Güiers area, with steeper slopes and a well-defined water body.

A DEM (figure $5(b)$ ) and satellite images with moderate and high spatial resolution were available. The DEM, which has 3 arcsec spatial resolution, corresponds to digital topography built by the National Aeronautics and Space Agency (NASA) and the National Geospatial-Intelligence Agency (NGA), within their joint project Shuttle Radar Topography Mission (SRTM; Farr et al. 2007). From the DEM, the raster maps of drainage directions and accumulated flow were computed, which are used

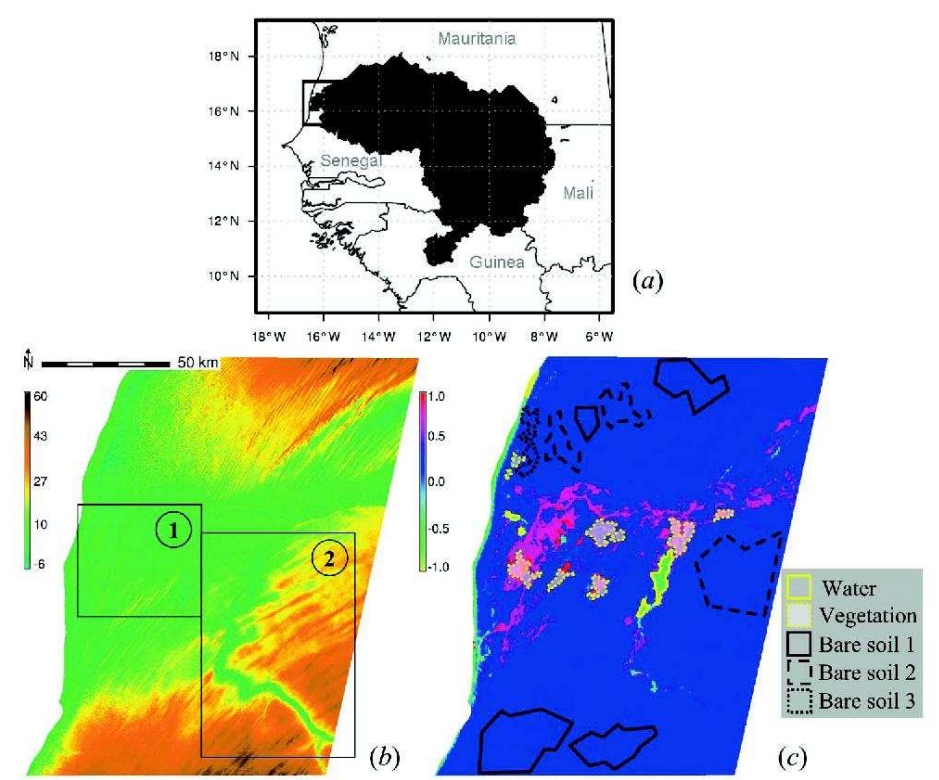

Figure 5. Study area: (a) location map, (b) DEM of Senegal River Valley with (1) wetlands in the Parc de Djoudj and (2) region of Lac de Güiers and (c) NDVI map showing the training areas. 
by the DOD algorithm. Surface reflectance images from the Moderate Resolution Imaging Spectroradiometer (MODIS) sensor dated 12 January 2002 (MOD09GHK product) have been used. The high multispectral and temporary resolution (1-2 days) makes the MODIS optical sensor one of the most advanced devices for monitoring the earth. Finally, the study has used information from the near-infrared and visible bands from two adjacent Landsat 7 Enhanced Thematic Mapper Plus (ETM+) images of 12 January 2002 (path-row 205-48 and 205-49). Since they were simultaneously captured with the MODIS image and have finer spatial resolution, the Landsat images are an appropriate tool to estimate the accuracy achieved with classification processes carried out with the MODIS image.

\subsection{Classified maps from satellite images}

7.2.1 Reference classified map from Landsat image. The Landsat image was processed using an unsupervised classification procedure, which identified nine representative land cover classes based on the visible and near-infrared bands. Two classes were merged to create the 'water' class, whilst the remaining classes defined the 'dry land' class. Given its spatial resolution ( $30 \mathrm{~m}$ ), the Landsat classification (see figure 8(a)) was the reference map to validate the goodness of the classification procedures applied on the MODIS image: a traditional supervised classification, and the proposed SA.

7.2.2 Supervised classified map from MODIS image. In the supervised classification procedure of the MODIS image, five bands were selected (B1, B2, B4, B6 and B7). The training areas (in figure $5(c)$ ) were identified with the normalized difference vegetation index (NDVI) map, together with colour composites between the selected bands. Next, five pure classes or endmembers were identified: the first class defined the 'water' or 'flooded' class, whilst the four remaining classes were merged in order to create the 'dry land' class, the 'vegetation' class and three 'bare soil' classes. The spectral signature file was computed using the GRASS GIS command i.gensig, while the final classified map was built with the i.maxlik command. The supervised classified map of the MODIS image preserves the spatial resolution from the original satellite images $(500 \mathrm{~m})$. Figure $8(b)$ shows the classified map with this procedure.

7.2.3 Sub-pixel level classified map from MODIS image. The sub-pixel level classification was carried out following the flow chart in figure 1 . The selected bands and the training areas are the same as in the supervised classification procedure, therefore the spectral signature file also corresponds. Once the pure classes have been defined, then the SA procedure will consist of two stages: in the first stage, the land cover fraction maps are built (using the isubpixel command) following the LMM procedure detailed before. Figure 6 shows the land cover fraction maps of the 'water' and 'vegetation' classes. The 'water' fraction map has high values on the Lac de Güiers area, on the Parc de Djoudj wetlands, on the Senegal river and on the coastline. On the other hand, the 'vegetation' class has high values on the cultivated area downstream of Lac de Güiers, and on the river banks.

The second stage of the SA procedure corresponds to the spatial arrangement of sub-pixels. Figure 7 shows the zoom in the Lac de Güiers area, between Saint Louis and Louga districts, which has the map evolution during the classification process: in figure $7(a)$ the classified map after the SCA procedure (i.subpixel command) can 


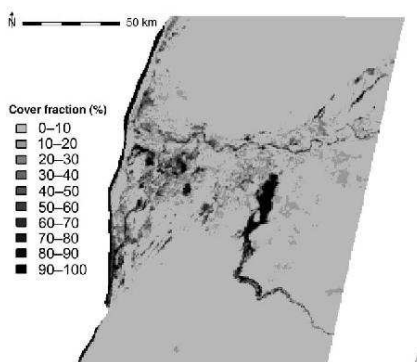

(a)

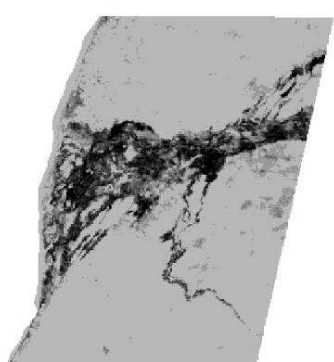

(b)

Figure 6. Raster maps of land cover fractions for ( $a$ ) 'flooded' class and (b) 'vegetation' class.

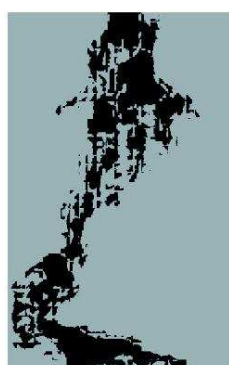

(a)

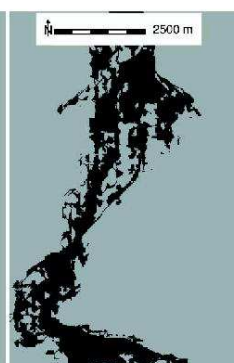

(b)

Dry

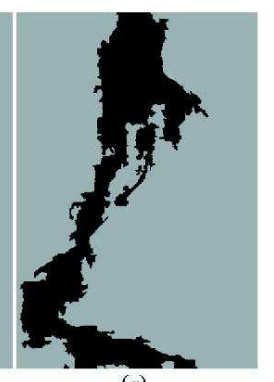

(c)

Flooded

Figure 7. Sub-pixel analysis method: $(a)$ level 0 classified map, $(b)$ level 1 classified map and (c) final classified map.

be seen; figure $7(b)$ shows the outcome map after applying the DOD algorithm that identifies new flooded cells on the drainage path (i.dod command). Finally, figure $7(c)$ shows the classified map after the ICI procedure (command $i$ isolated), which identifies and reclassifies the isolated small clusters.

The final classified map using the SA procedure can be seen in figure $8(c)$. The developed GRASS GIS commands, which run the SA classification, can split a coarse pixel in to $10 \times 10$ finer sub-pixels, therefore the classified map with the new methodology, using MODIS images, has $50 \mathrm{~m}$ of spatial resolution.

\subsection{Results}

The results of the SA classification were evaluated against the Landsat classified map with spatial resolution modified to $50 \mathrm{~m}$. Both the classification accuracy and the kappa index, $\kappa$, in the whole study area and in the regions, detailed in figure 5, were estimated using confusion matrices. The confusion matrices are classification quality indicators (Kohavi and Provost 1998), while the kappa index assesses the proportion 


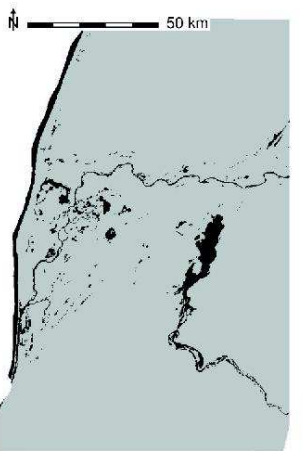

(a)

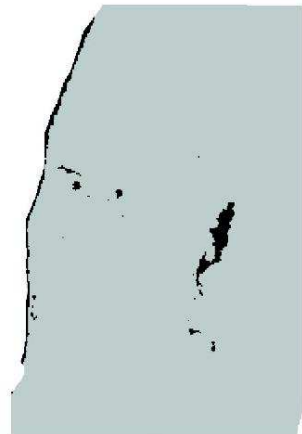

(b)

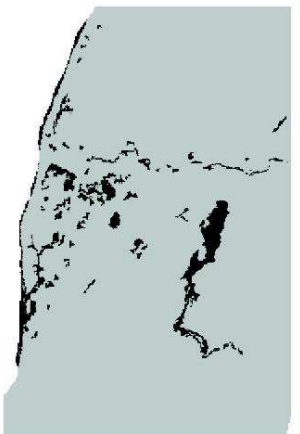

(c)

Dry

Flooded

Figure 8. Flood maps from several images and methodologies: $(a)$ unsupervised classification of Landsat image, $(b)$ supervised classification of MODIS image and $(c)$ classification at subpixel level with MODIS image.

of observed agreements above the expected value of agreements by chance (Cohen 1960).

It can be seen from the confusion matrix in the whole study area (table 1) that the SA methodology considerably improves the mapping of flooded areas $(79.9 \%)$ as compared to the supervised classification $(39.4 \%)$. The dry land is better mapped by the supervised classification $(99.98 \%$ ) because the flooded cells are poorly identified. The SA manages to correctly classify $96.8 \%$ of dry land. The computed kappa index for supervised classification is $\kappa=0.55$ (95\% confidence interval $= \pm 0.0019$ ), whilst the SA kappa index is $\kappa=0.60( \pm 0.0014)$, therefore the better classification from the proposed method is confirmed. Figure 9 depicts the results in the whole scenario using both the supervised classification and the SA procedure.

Two regions were detailed which have flooded areas with different characteristics. Region 1 (figure 5, Parc de Djoudj) is inundated by the river seasonal flood; the accurate mapping of flooded areas is difficult because they are randomly located and

\begin{tabular}{|c|c|c|c|c|}
\hline & Dry (cells) & Water (cells) & Dry $(\%)$ & Water $(\%)$ \\
\hline \multicolumn{5}{|c|}{ Supervised classification } \\
\hline $\begin{array}{l}\text { Dry } \\
\text { Water }\end{array}$ & $\begin{array}{r}7414791 \\
209809\end{array}$ & $\begin{array}{r}1582 \\
136318\end{array}$ & $\begin{array}{l}99.98 \\
60.62\end{array}$ & $\begin{array}{r}0.02 \\
39.38\end{array}$ \\
\hline \multicolumn{5}{|c|}{ Sub-pixel analysis } \\
\hline $\begin{array}{l}\text { Dry } \\
\text { Water }\end{array}$ & $\begin{array}{r}7093093 \\
61628\end{array}$ & $\begin{array}{l}232844 \\
244635\end{array}$ & $\begin{array}{l}96.82 \\
20.12\end{array}$ & $\begin{array}{r}3.18 \\
79.88\end{array}$ \\
\hline
\end{tabular}

Notes: Columns, assigned classes (MODIS image); rows, reference data (Landsat image). 


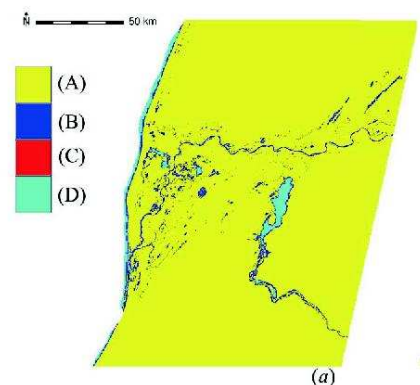

(a)

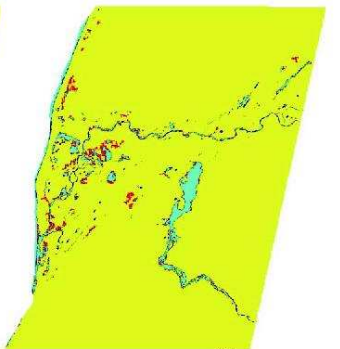

(b)

Figure 9. Classification results for the whole study area: $(a)$ supervised classification, and (b) sub-pixel analysis. Notations: (A) dry land correctly mapped; (B) flooded areas incorrectly mapped as dry land; (C) dry land incorrectly mapped as flooded; and (D) flooded areas correctly mapped. Classes (B) + (D) are the flooded areas mapped with Landsat image.

Table 2. Confusion matrices in region 1.

\begin{tabular}{lrccr}
\hline & Dry (cells) & Water (cells) & Dry $(\%)$ & Water (\%) \\
\hline \multicolumn{2}{l}{ Supervised classification } & & & \\
Dry & 907470 & 418 & 99.95 & 0.05 \\
Water & 62630 & 9482 & 86.85 & 13.15 \\
Sub-pixel analysis & & & \\
Dry & 809894 & 97994 & 89.21 & 10.79 \\
Water & 16870 & 55242 & 23.39 & 76.61 \\
\hline
\end{tabular}

Notes: Columns, assigned classes (MODIS image); rows, reference data (Landsat image).

the terrain is very flat. In contrast, region 2 (figure 5, Lac de Güiers) is a perennial water body, with a well-defined shoreline and steeper terrain slopes.

In region 1, the supervised classification has a poor performance (see table 2). It only identifies $13.2 \%$ of the flooded areas, while the SA procedure achieves $76.6 \%$. However, the supervised classification identifies almost all dry land areas (99.9\%), whilst the SA classification shows a worse performance in this region $(89.2 \%)$. This is because the supervised classification procedure poorly identifies the flooded areas. The accuracy of the classification processes can be computed from the confusion matrix (Kohavi and Provost 1998): the supervised procedure achieved 93.6\%, while the SA procedure obtained $88.3 \%$. The computed kappa index in the supervised classification is $\kappa=0.22( \pm 0.0059)$, whilst the SA has a kappa value of $\kappa=0.43( \pm 0.0031)$, therefore the high agreement between dry areas in the traditional methodology is by chance.

Table 3 shows the results obtained in region 2. The mapping of flooded areas was better by the two methodologies $(52.8 \%$ by traditional supervised classification and $87.9 \%$ by the SA procedure). Once again, almost all dry land areas were correctly identified by the traditional method, but the kappa index shows a better mapping with the SA procedure too ( $\kappa=0.68 \pm 0.0028$ and $\kappa=0.79 \pm 0.0019$, respectively). 
J. D. Giraldo Osorio and S. G. García Galiano

Table 3. Confusion matrices in region 2.

\begin{tabular}{|c|c|c|c|c|}
\hline & Dry (cells) & Water (cells) & Dry $(\%)$ & Water $(\%)$ \\
\hline \multicolumn{5}{|c|}{ Supervised classification } \\
\hline Dry & 1909851 & 577 & 99.97 & 0.03 \\
\hline Water & 50249 & 56123 & 47.24 & 52.76 \\
\hline \multicolumn{5}{|c|}{ Sub-pixel analysis } \\
\hline Dry & 1862044 & 34124 & 98.20 & 1.80 \\
\hline Water & 12821 & 93511 & 12.06 & 87.94 \\
\hline
\end{tabular}

Notes: Columns, assigned classes (MODIS image); rows, reference data (Landsat image).

\section{Conclusions}

The present work shows the huge potential of SA to identify land cover from satellite images. The proposed SA methodology is able to improve the spatial resolution of classified maps from the input satellite images. The technique has been applied with MODIS images with moderate spatial resolution, however it could be run with images from any sensor or with any treatment (e.g. the images which are the output from a principal component analysis, or multi-frequency microwave images) which meet the spatial dependence hypotheses.

The LMM algorithm introduced to solve the LMM inside the coarse pixels is well considered. Several authors have proposed other methodologies which are marginally more accurate, but which imply high computational costs that advise against their application. This simple procedure proposed is also justified because the flooded area identification is modified across the SA stages using the study zone DEM (it is included in the cost matrix estimate in the SCA procedure, and in the mapping of new flooded areas with the DOD algorithm), or reclassifying the spurious small clusters with the ICI procedure.

The efficient mapping of flooded flat areas has been demonstrated. The developed procedure obtains a 'soft' classification with improved spatial resolution because it extracts information at the sub-pixel level from satellite images. The procedure merges remote-sensing techniques with DEM data and geostatistical analysis in order to improve the flooded area mapping. The SCA procedure adds the study zone DEM to encourage the placing of flooded sub-pixels on the lower terrain areas. The drainage path map and the accumulated flow map, both of which are provided by the DEM, are used to identify new flooded cells with the DOD algorithm. Finally, the ICI procedure 'cleans' the final classified map.

The SA has been applied on the lower Senegal River Valley, showing better classification results of flooded areas than a traditional supervised classification procedure that used the same input satellite images with moderate spatial resolution. The accuracy of the SA procedure to identify flooded areas is noticeably better, although the dry land areas are better identified by the supervised classification. However, the computed kappa indices demonstrate that the SA achieves a more consistent mapping of 'flooded areas'-'dry land' set than the traditional algorithm. It has been proven in two different scenarios: a scenario with seasonal floods, which are difficult to detect due to the flat terrain; and a scenario with a perennial lake and steeper terrain that defines better the water body. In both cases, the percentages of flooded areas correctly 
identified and the computed kappa index show a better classification from the SA algorithm.

The introduced methodology shows some uncertainties which are justified with both the moderate spatial resolution and inaccurate study zone DEM. It would be desirable for the DEM to have the same spatial resolution as the output classified maps from the SA procedure, in order to guarantee the correct agreement between both the DEM and the sub-pixels location. According to Sanders (2007), the SRTM DEM could be contaminated by radar speckle, which adds noise and undulations on flat surfaces. The model inaccuracies give rise to unreal pool systems, which drain randomly, given the poor representation of drainage directions on these areas. In the SA proposed method, this error could be propagated through its several stages: the SCA procedure will locate the flooded sub-pixels on the unreal pools, while the ICI procedure will expand the unreal flooded area from these pools. Also, the unsuitable representation of drainage paths, produced by DEM inaccuracy, will lead to an overestimation of flooded area and, therefore, an underestimation of dry land area. Figure 9 represents this fact, particularly in region 1 , where several unreal pools that reduce the performance to define non-flooded areas were identified.

Finally, it should be emphasized that the developed computer tool is based on an open source code tool, under GNU licence. This characteristic, together with the use of free satellite images, encourages its application by stakeholders in developing countries.

\section{Acknowledgments}

This work was performed within the framework of the AMMA project. Based on

a French initiative, AMMA has been constructed by an international group and is currently funded by large number of agencies, especially from France, the UK, the USA and Africa. It has been the beneficiary of a major financial contribution from the European Community's Sixth Framework Research Programme. Detailed information on the scientific coordination and funding is available on the AMMA international web site (https://www.amma-eu.org). We appreciate the R\&D Project CGL2008-02530/BTE of the Spanish Ministry of Science and Innovation support.

\section{References}

Aban, J.E.L., Tsolmon, R. and TAteishi, R., 2002, Linear mixing model based on optimization method for land cover mapping using LANDSAT ETM+ and SPOT-HRV data. In Proceedings of Geoscience and Remote Sensing Symposium. IEEE International, 6, pp. $3483-3485$

Andersen, J., REFsgaArd, J. and JEnsen, K.H., 2001, Distributed hydrological modelling of the Senegal river basin - model construction and validation. Journal of Hydrology, 247, pp. 200-214.

BENGER, S., 2003, Remotely sensed determination of flood surface gradients for hydrological modelling of semi-arid floodplains. In Proceedings of Geoscience and Remote Sensing Symposium. IEEE International, 4, pp. 2950-2952.

Brivio, P., Colombo, R., Maggi, M. and Tomasoni, R., 2002, Integration of remote sensing data and GIS for accurate mapping of flooded areas. International Journal of Remote Sensing, 23, pp. 429-441.

COHen, J., 1960, A coefficient of agreement for nominal scales. Educational and Psychological Measurement, 20, pp. 37-46.

Farr, T.G., Rosen, P.A., Caro, E., Crippen, R., Duren, R., Hensley, S., Kobrick, M., Paller, M., Rodríguez, E., Roth, L., Seal, D., Shaffer, S., Shimada, 
J., UMland, J., Werner, M., Oskin, M., Burbank, D. and Alsdorf, D., 2007 , The shuttle radar topography mission. Reviews of Geophysics, 45, p. RG2004, doi:10.1029/2005RG000183.

Giacomelit, A., Mancini, M. and Rosso, R., 1997, Integration of ERS 1 PRI imagery and digital terrain models for the assessment of flooded areas. In ESA Proceedings of the 3rd ERS Symposium, Florence, Italy. Available online at: http://earth.esa. int/workshops/ers $97 /$ papers/mancini/249c.htm

Hongen, Z., Suhong, L., QizHong, L. and JiAncheng, S.J., 2004, Sub-pixel lake mapping in Tibetan Plateau. In Proceedings of Geoscience and Remote Sensing Symposium. IEEE International, 5, pp. 3073-3076.

Hu, Y.H., LeE, H.B. and SCArpace, F.L., 1999, Optimal linear spectral unmixing. IEEE Transactions on Geoscience and Remote Sensing, 37, pp. 639-644.

JiAnghong, S., Zhongming, Z., Qingye, Z. and Yangeng, W., 2004, An algorithm for eliminating the isolated regions based on connected area in image classification. In Proceedings of Geoscience and Remote Sensing Symposium. IEEE International, 5, pp. 3058-3061.

Kàliainen, H., Bosdogiani, P., Petrou, M. and Kittler, J., 1996, Mixed pixel classification with randomized Hough transform. In Proceedings of 13 th International Conference on Pattem Recognition, 2, pp. 576-580.

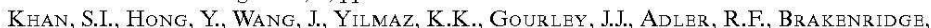
G.R., Policelli, F., HABIB, S. and Irwin, D., 2011, Satellite remote sensing and hydrologic modeling for flood inundation mapping in Lake Victoria Basin: implications for hydrologic prediction in ungauged basins. IEEE Transactions on Geoscience and Remote Sensing, 49, pp. 85-95.

Kim, C. and CHI, K., 1998, Flood damage mapping in North Korea using multisensor data. In Proceedings 19th Asian Conference of Remote Sensing, 16-20 November 1998, Manila, Philippines. Available online at: http//www.a-a-r-s.org/acrs/ proceeding/ACRS1998/Papers/DIS98-1.htm

KLein GebBinck, M.S. and SChouten, T.E., 1995, Decomposition of mixed pixels based on a physical linear mixture model. Image and GIS Processing for Remote Sensing II, SPIE 2579 , pp. 104-115.

Kohavi, R. and Provost, F., 1998, Glossary of terms. Editorial for the special issue on application of machine learning and the knowledge of discovery process. Machine Learning, 30, pp. 271-274.

Lucas, N.S., Shanmugam, S. and Barnstey, M., 2002, Sub-pixel mapping of a costal dune ecosystem. Applied Geography, 22, pp. 253-270.

Martinez, J.M. and Le ToAn, T., 2007, Mapping of flood dynamics and spatial distribution of vegetation in the Amazon floodplain using multitemporal SAR data. Remote Sensing of Environment, 108, pp. 209-223.

Nyborg, L. and SANDHOLt, I., 2001, NOAA-AVHRR based flood monitoring. In Proceedings of Geoscience and Remote Sensing Symposium. IEEE 2001 International, 4, pp. 1696-1698.

Profeti, G. and Macintosh, H., 1997, Flood management through Landsat TM and ERS SAR data: a case study. Hydrological Processes, 11, pp. 1397-1408.

Saici, P., Thompson, J. and Rebelo, L., 2001, Monitoring wetland extent and dynamics in the Cat Tien National Park, Vietnam, using space-based radar remote sensing. In Geoscience and Remote Sensing Symposium. IEEE 2001 International, 7, pp. 3099-3101.

SAkamoto, T., VAn NguYen, N., Ohno, H., Ishitsuka, N. and Yokozawa, M., 2005, Spatio temporal distribution of rice phenology and cropping systems in the Mekong Delta with special reference to the seasonal water flow of the Mekong and Bassac rivers. Remote Sensing of Environment, 100, pp. 1-16.

SANDERS, B.F., 2007, Evaluation of on-line DEMs for flood inundation modelling. Advances in Water Resources, 30, pp. 1831-1843. 
SANYAL, J. and Lu, X., 2004, Application of remote sensing in flood management with special reference to monsoon Asia: a review. Natural Hazards, 33, pp. 283-301.

SANYAL, J. and LU, X., 2005, Remote sensing and GIS-based flood vulnerability assessment of human settlements: a case study of Gangetic West Bengal, India. Hydrological Processes, 19, pp. 3699-3716.

Sмiтн, L., 1997, Satellite remote sensing of river inundation area, stage and discharge: a review. Hydrological Processes, 11, pp. 1427-1439.

TowsEND, P.A. and WALSH, S.J., 1998, Modeling floodplain inundation using an integrated GIS with radar and optical remote sensing. Geomorphology, 21, pp. 295-312.

TOYrAA, J. and Pietroniro, A., 2005, Towards operational monitoring of a northern wetland using geomatics-based techniques. Remote Sensing of Environment, 97, pp. 174-191.

Tralli, D.M., Blom, R.G., Zlotnicki, V., Donnellan, A. and Evans, D.L., 2005, Satellite remote sensing of earthquake, volcano, flood, landslide and coastal inundation hazards. ISPRS Journal of Photogrammetry and Remote Sensing, 59, pp. 185-198.

Verhoeye, J. and De Wulf, R., 2000, Sub-pixel Mapping of Sahelian Wetlands using Multi-temporal SPOT VEGETATION Images. In VEGETATION 2000 Conference, 3-6 April 2000, Lake Maggiore, Italy. Available online at: http://www.spot-vegetation. com/pages/vgtprep/vgt2000/verhoeye.html

VERHOEYE, J. and DE WULF, R., 2002, Land cover mapping at sub-pixel scales using linear optimization techniques. Remote Sensing of Environment, 79, pp. 96-104.

WANG, Y., 2002, Mapping extent of floods: what we have learned and how we can do better. Natural Hazards Review, 3, pp. 68-73.

WANG, Y., 2004, Using Landsat 7 TM data acquired days after a flood event to delineate the maximum flood extent on a coastal floodplain. Intemational Joumal of Remote Sensing, 25, pp. 959-974.

WANG, Y., Colby, J.D. and MulcAhy, K.A., 2002, An efficient method for mapping flood extent in a coastal floodplain using Landsat TM and DEM data. International Joumal of Remote Sensing, 23, pp. 3681-3696.

YANG, C., ZHOU, C. and WAN, Q., 1999, Deciding the flood extent with Radarsat SAR data and Image Fusion. In Proceedings of 20 th Asian Conference of Remote Sensing, 22-25 November 1999, Hong Kong. Available online at: http://www.a-a-r-s.org/acrs/ proceeding/ACRS1 999/Papers/PS399-13.htm

Zhan, $\mathrm{X}$., Defries, R., Townshend, J., Dimiceli, C., Hansen, M., Huang, C. and SoHlberg, R., 2000, The $250 \mathrm{~m}$ global land cover change product from the moderate resolution imaging spectroradiometer of NASA's Earth Observation System. International Journal of Remote Sensing, 21, pp. 1433-1460.

Zhan, X., Sohlberg, R., Townshend, J., Dimiceli, C., Carroll, M., Eatsman, J., Hansen, M. and DEFriES, R., 2002, Detection of land cover changes using MODIS $250 \mathrm{~m}$ data. Remote Sensing of Environment, 83, pp. 336-350. 



\subsection{Resumen del Capítulo de Libro Internacional}

\subsubsection{Título}

Non stationary analysis of spatial patterns of extreme rainfall events in West Africa.

\subsubsection{Objetivos específicos}

- Contrastar los resultados para evaluar la confiabilidad de los diferentes RCMs considerados, provistos por el Proyecto Europeo ENSEMBLES.

- Comparar la tendencia futura de las precipitaciones máximas, con otros trabajos realizados por los autores, en los cuales se aplicaron otras técnicas ensembles para construir las distribuciones conjuntas de precipitaciones máximas diarias.

- Confrontar y discutir el trabajo realizado, en un Congreso de alto nivel internacional. Por lo que ha sido presentado de forma oral en 25th International Union of Geodesy and Geophysics General Assembly, IUGG 2011 (Melbourne, Australia).

\subsubsection{Metodología}

El objetivo de este trabajo es construir mapas de precipitación diaria máxima anual (AMDR) para varios periodos de retorno para la cuenca del Río Senegal. Sin embargo, los análisis estacionarios tradicionales no son adecuados, dadas las tendencias significativas que han sido detectadas en las series hidrometeorológicas históricas. En consecuencia, se ha aplicado la herramienta GAMLSS (Rigby and Stasinopoulos, 2005) para ajustar los parámetros de las funciones de distribución de probabilidad (PDF) no estacionarias. Las series temporales de AMDR fueron obtenidas desde mallas de precipitación diaria, interpoladas de datos observados del IRD o construidas con los Modelos Climáticos Regionales (RCM) seleccionados. La amplia divergencia en las tendencias pronosticadas desde los RCM obliga a la utilización de PDF ensemble, que son construidas usando técnicas de bootstrapping (Efron and Tibshirani, 1993).

\subsubsection{Resultados}

En la construcción de las PDF ensemble se ha dado mayor peso a los RCMs que presentaron un menor desvío, y este peso se conservó constante para toda el área de estudio.

Se construyeron mapas de diversos estadísticos (media, desviación estándar y distintos cuantiles), interpolando los valores de la PDF ensemble construida en cada sitio de análisis. Para los años de referencia 1990 y 2050, se calculó la diferencia entre los mapas para observar el cambio en la distribución del patrón espacial de las AMDR. 
Se esperan incrementos significativos de la media y la desviación estándar de las AMDR en el Valle del Río Senegal. Este cambio en las características medias de las AMDR se verá reflejado en un cambio significativo en las AMDR asociadas con un periodo de retorno de 10 años en la parte baja de la cuenca.

\subsubsection{Conclusiones}

El análisis del desvío de los datos de los RCMs permitió establecer las habilidades de cada uno de ellos para modelar las AMDR. Sin embargo, este mismo análisis reveló que no existe un modelo ideal para toda el área de estudio, por lo que deben construirse PDF ensemble para explotar toda la información suministrada por los modelos. Desde los mapas interpolados de las PDF ensemble, se espera un aumento significativo de las AMDR en el Valle del Río Senegal. 


\subsubsection{Copia de la publicación}

\section{Non-stationary analysis of spatial patterns of extreme rainfall events in West Africa}

\section{SANDRA G. GARCÍA GALIANO \& JUAN D. GIRALDO OSORIO}

Technical University of Cartagena, R\&D Group of Water Resources Management, Department of Civil Engineering, Paseo Alfonso XIII 52, 30203, Cartagena, Spain

sandra.garcia $(\bar{a})$ upct.es; juan.giraldo $(a)$ upct.es

Abstract Heavy storm events frequently cause extensive damage, and often result in loss of life and property. The objective of this work is to build maps of Annual Maximum Daily Rainfall (AMDR) fo various return periods for the Senegal River Basin. However, traditional stationary analyses are not suitable, since meaningful trends have been detected in historical hydrometeorological time series. Therefore, the GAMLSS (Generalized Additive Models for Location, Scale and Shape) tool is applied to fit the parameters of the probability density functions (pdfs). AMDR time series were estimated using observed daily rainfall (RCMs). The wide divergence in predicted trends from RCMs impose the use of ensemble pdfs, which can be built using bootstrapping techniques. The plausible AMDR maps associated with various quantiles, interpolated from these ensemble pdfs, could be used by stakeholders to develop strategies of mitigation and adaptation to climate change impacts on floods events.

Key words non-stationarity; GAMLSS; regional climate models; bootstrapping; ensemble probability density function; maximum daily rainfall

\section{INTRODUCTION}

The increase in rainfall and evaporation rates, caused by global warming in some areas of the Earth, has theoretically become a threat to the population, given the increased frequency of extreme events (Kundzewicz et al., 2007). Moreover, these threats affect the developing countries unequally, where resources for adaptation measures and mitigation are limited (Huntington, 2006).

In particular, the Senegal River Valley (West Africa) is an area where land degradation and loss of vegetation cover is a persistent problem, due to terrain aridity and poor agricultural practices and grazing (Lebel et al., 2009). In the future, these environmental problems will be exacerbated by severe floods associated with higher maximum rainfall. The hydrological systems in this region are affected by different drivers (climate change, land-use changes, demographic growth, among others), which do not allow one to assert that stationary conditions can be valid. Therefore, a non-stationary probabilistic model capable of reproducing the existing hydroclimatic variability should be used (Milly et al., 2008).

Currently, regional climate models (RCM) constitute a tool for simulation of plausible climate scenarios, with a spatial resolution suitable for studies addressing the impact of climate change at the basin scale. In conjunction with Generalized Additive Models for Location, Scale and Shape (GAMLSS), it is possible to simulate the non-stationarity of the probability density functions (pdfs) of maximum rainfall and to obtain a measure of the uncertainty of change. However, due to the divergence in the results obtained from different RCMs, the use of ensemble techniques is needed to simulate the variability in precipitation.

The aim of the present study is the assessment of change in the pdfs of the annual maximum daily rainfall (AMDR) on the Senegal River Basin (West Africa), and the spatial pattern associated with the change. The first section describes the database used and the study area. Then, the procedure to be followed to identify the skill score of each RCM in ensemble building pdf for the sites is described. The results and discussion section presents a brief analysis of the maps constructed, and finally the findings of the work.

\section{STUDY AREA AND DATA}

The study area consists of the Senegal River Basin (Fig. 1), which presents a strong decreasing gradient of precipitation in a south-north direction. This corresponds to the eco-regions Guinean 
Forest Savanna (GFS), West Sudanian Savanna (WSS) and Sahelian Acacia Savanna (SAS), according to Olson et al. (2001). The main resources of the basin lie in the mountains of Fouta Djalon within GFS, where rainfall can exceed $2000 \mathrm{~mm} / \mathrm{year}$, while in the Sahel arid region there are areas with precipitation of less than $200 \mathrm{~mm} /$ year (Andersen et al., 2001).

The AMDR series were obtained from the observed daily rainfall grids and from RCMs provided by the European ENSEMBLES Project (Christensen et al., 2009). The observed series compiled by the IRD (Institut de Recherche pour le Developpement; previously ORSTOM, France) have been widely used in other studies of high-frequency cycles of rainfall in West Africa (Diedhiou et al., 1999; Janicot \& Sultan, 2001; Messager et al., 2004; Paeth et al., 2005; García \& Giraldo, 2010). The IRD database has spatial resolution of $1^{\circ}$, and constitutes the baseline data for the bias analysis based on empirical cumulative distribution functions (cdfs), in spite of lacks of spatial continuity, which imposes restriction on the analyses (Fig. 1(a)). Because the RCMs have spatial continuity, for the analysis 120 sites on a regular grid of $1^{\circ} \times 1^{\circ}$ (Fig. 1(a)) were selected. However, the spatial discontinuity of IRD data constrains the bias analysis to only 43 of these sites (Fig. 1(b)). The main features of the RCMs used in conjunction with IRD data, are presented in Table 1. The selection of RCMs was based on their temporal coincidence with the IRD data (period 1970-1990), which enables the bias analysis. A detailed description of the RCMs used in this study is presented by Christensen et al. (2009).

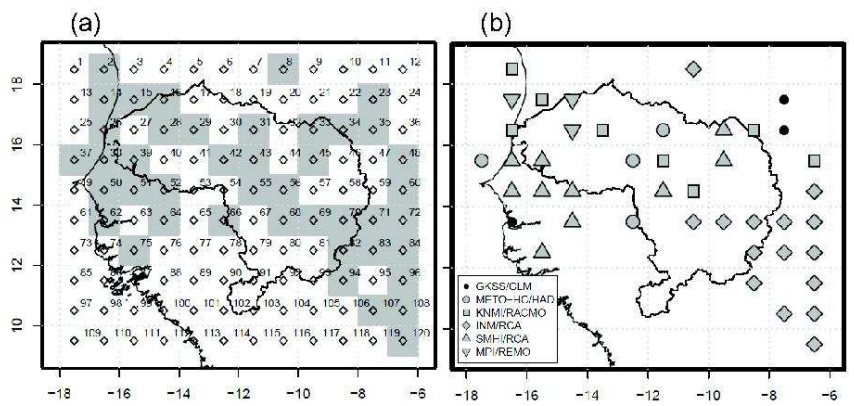

Fig. 1 Senegal River Basin and the results of the bias analysis: (a) sites selected for analyses. The IRD sites are highlighted with gray squares; and (b) results of AMDR bias analysis. Markers indicate the RCMs which best fit the actual AMDR cdf in each site with IRD time series.

Table 1 Summary of characteristics of the IRD data and selected RCMs.

\begin{tabular}{lllll}
\hline Name & Institute & GCM & RCM & Temporal coverage \\
\hline IRD & & & & $1970-1990$ \\
GKSS/CLM & GKSS $^{(1)}$ & ECHAM5 & CLM & $1961-2050$ \\
METO-HC/HAD & HC $^{(2)}$ & HadCM3Q0 & HadRM3P & $1951-2099$ \\
KNMIRACMO & KNMI $^{(3)}$ & ECHAM5-r3 & RACMO & $1970-2050$ \\
INM/RCA & INM $^{(4)}$ & HadCM3Q0 & RCA & $1951-2099$ \\
SMHI/RCA & SMHI $^{(5)}$ & HadCM3Q0 & RCA & $1951-2100$ \\
MPI/REMO & MPI $^{(6)}$ & ECHAM5-r3 & RACMO & $1950-2050$
\end{tabular}

(1) Institute for Coastal Research, Germany; ${ }^{(2)}$ Hadley Centre, UK; ${ }^{[3}$ Royal Netherlands Meteorological Institute: ${ }^{(1)}$ Institute for Coastal Research, Germany; ${ }^{(2)}$ Hadley Centre, UK; ${ }^{(3)}$ Royal Netherlands Meteorological Institute;
Institute, Institute of Meteorology, Spain; ${ }^{(5)}$ Swedish Meteorological and Hydrological Institute; ${ }^{(6)}$ Max Planck

\section{METHOD}

Bias analysis

Due to the spatial discontinuity of IRD data, the bias analysis was restricted to 43 sites as shown in Fig 1(a). For each of these sites, cdfs were constructed using AMDR series obtained from daily 
IRD data and RCM databases, for the period 1970-1990. The bias analysis was carried out through computing the p-value of the two-sample Kolmogorov-Smirnov goodness-of-fit test between the AMDR cdf from IRD and the selected RCMs (Sheskin, 2000; Marques de Sa, 2003).

The summary of the results of bias analysis is presented in Fig. 1(b). The INM/RCA was the RCM with a better skill in 15 of the 43 bias analysis sites (grouped in the southeast part of the study area); followed by SMHI/RCA (10 sites, mostly in the west part of the study area); and the KNMI/RACMO (eight sites, distributed in the Senegal River Valley). The METO-HC/HAD (four sites), along with the GKSS/CLM and MPI/REMO (with three sites each), were the RCMs with less skill. The results of the bias analysis will be considered in the construction of the ensemble pdf for each site in the study area, giving greater weight to the RCMs with less bias.

\section{GAMLSS applied to non-stationary analysis}

To analyse the trend of the pdf associated with the AMDR series for each site, the Generalized Additive Models for Location Scale and Shape (GAMLSS) were used. In the analysis, four theoretical probability distributions of two parameters were considered: Gumbel (GU), gamma (GA), Lognormal (LN) and Weibull (WEI). According to Rigby \& Stasinopoulos (2005), and Stasinopoulos \& Rigby (2007), GAMLSS are semi-parametric regression models for adjusting the parameters of the pdf of the response variable (AMDR) as a function of an explanatory variable (time) using nonparametric smoothing functions. GAMLSS has proved useful in modelling the evolution of pdf parameters of nonstationary hydrological time series of peak flows (Villarini et al., 2009), temperatures (Villarini et al., 2010), and dry spells of rainfall (Karambiri et al., 2011). As an example, the results of the GAMLSS adjustment to the six RCMs considered are shown in Fig. 2 for site 28 . Figure 2 shows the temporal variation of the pdf of AMDR represented with curves for different percentiles $(5,10,25,50,75,90$ and 95\%). The goodness of fit to the statistical model was assessed by evaluating the normality of the residuals and visual inspections of the qq-plots and the worm plot (not shown), according to the methodology presented by van Buuren \& Fredriks (2001).

\section{Definition of skill scores for ensemble pdf}

As seen from Fig. 2, the GAMLSS adjustment to various series of AMDR in each site predicts trends that differ in magnitude and sign, depending on the RCM considered. Therefore, for the building of the ensemble pdf for each site, greater weight was given to the RCMs that showed a better performance in the bias analysis, using bootstrapping techniques (Efron \& Tibshirani, 1993). Random subsamples of size $N=1000$ of each RCM were built as they have had good performance in the bias analysis (e.g. 15 random samples of INM/RCA, for only three samples of GKSS/CLM or MPI/REMO). To obtain the evolution of the probability distribution of AMDR in each site, ensemble pdfs were constructed for each ten years in the period 1970-2050, and basic statistics (mean $\mu$ and standard deviation $\sigma$ ) and the quantiles for 5, 10, 25, 50, 75, 90 and 95\% with their respective confidence intervals $(95 \% \mathrm{CI})$ were estimated. The ensemble pdf built by applying this methodology for site 28 is shown in Fig. 3.

\section{RESULTS AND DISCUSSION}

Once the statistics for the ensemble pdf at each site have been obtained for the period 1970-2050, these values were interpolated to generate maps depicting the spatio-temporal evolution of the AMDR distribution. In Fig. 4, the maps constructed for the mean, standard deviation and for the $90 \%$ quantile (or $T r=10$ years) for 1990 and 2050 , and the percentage difference between them, are presented.

The maps constructed for $\mu$ (Fig. 4(a)) have a spatial structure that matches the latitudinal gradient of mean annual precipitation in the region, yet the difference between them does not preserve this structure. Significant increase in $\mu$ is expected in the Senegal River Valley and the 
78 Sandra G. Garcia \& Juan D. Giraldo
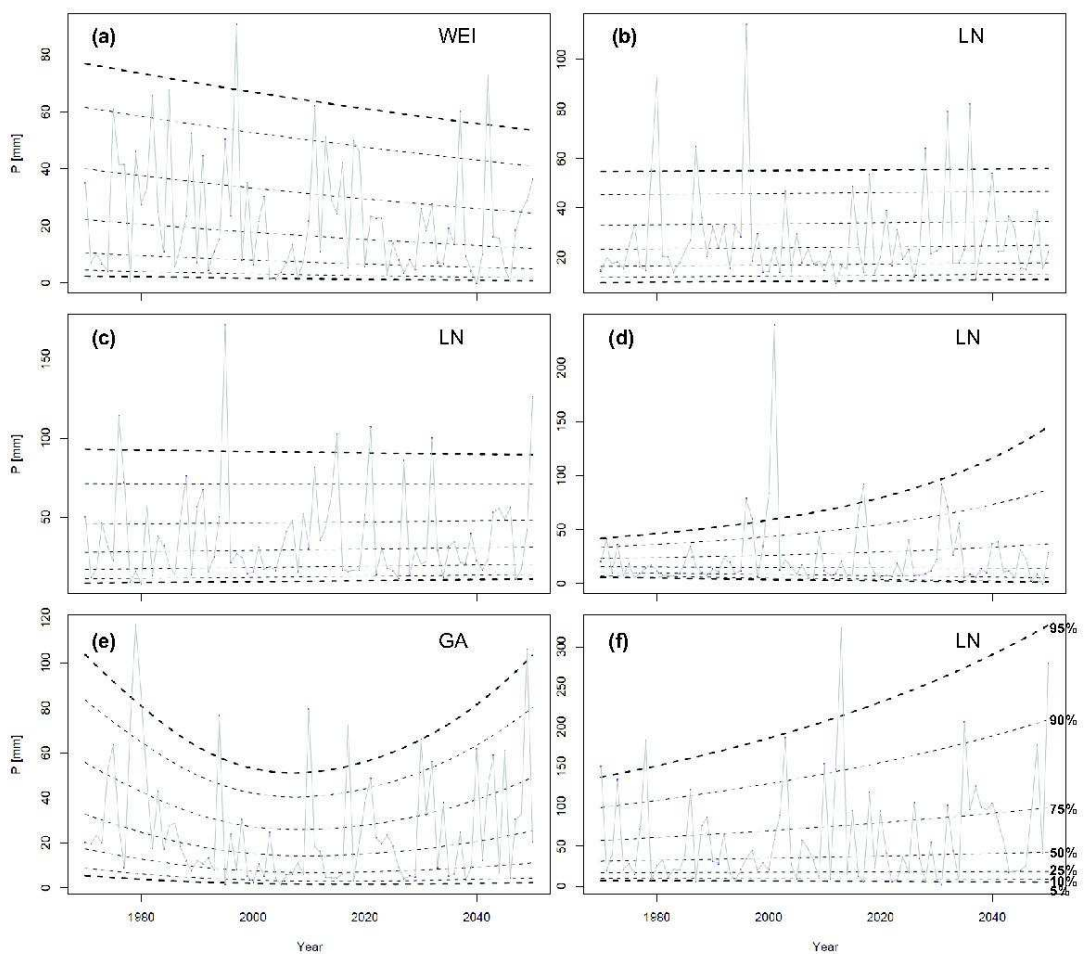

Fig. 2 GAMLSS analysis of AMDR for site 28 for several RCMs: (a) GKSS/CLM, (b) METOHC/HAD, (c) KNMI-RACMO, (d) INM-RCA, (e) SMHI/RCA, and (f) MPI-M-REMO. The centile curves $(5$ to $95 \%$ are represented by dashed lines. It should be noted that the ordinate scale is automatically fixed. The pdf used are gamma (GA), lognormal (LN) and Weibull (WEI).

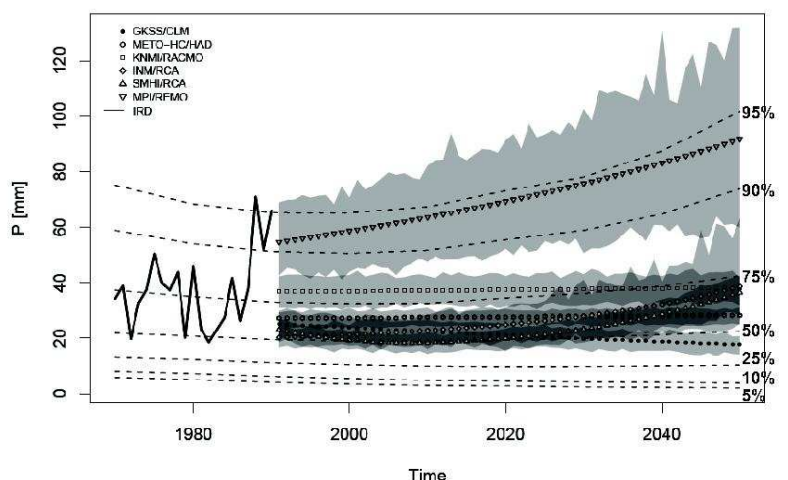

Fig. 3 Ensemble pdf for site 28 . The dashed lines show 5 to $95 \%$ quantiles of ensemble pdf, and markers represent RCMs mean values. The grey polygons show the mean variability in the period 1991-2050, using the 95\% CI computed with bootstrapping. The IRD AMDR series in 1970-1990 is presented as a solid line. 


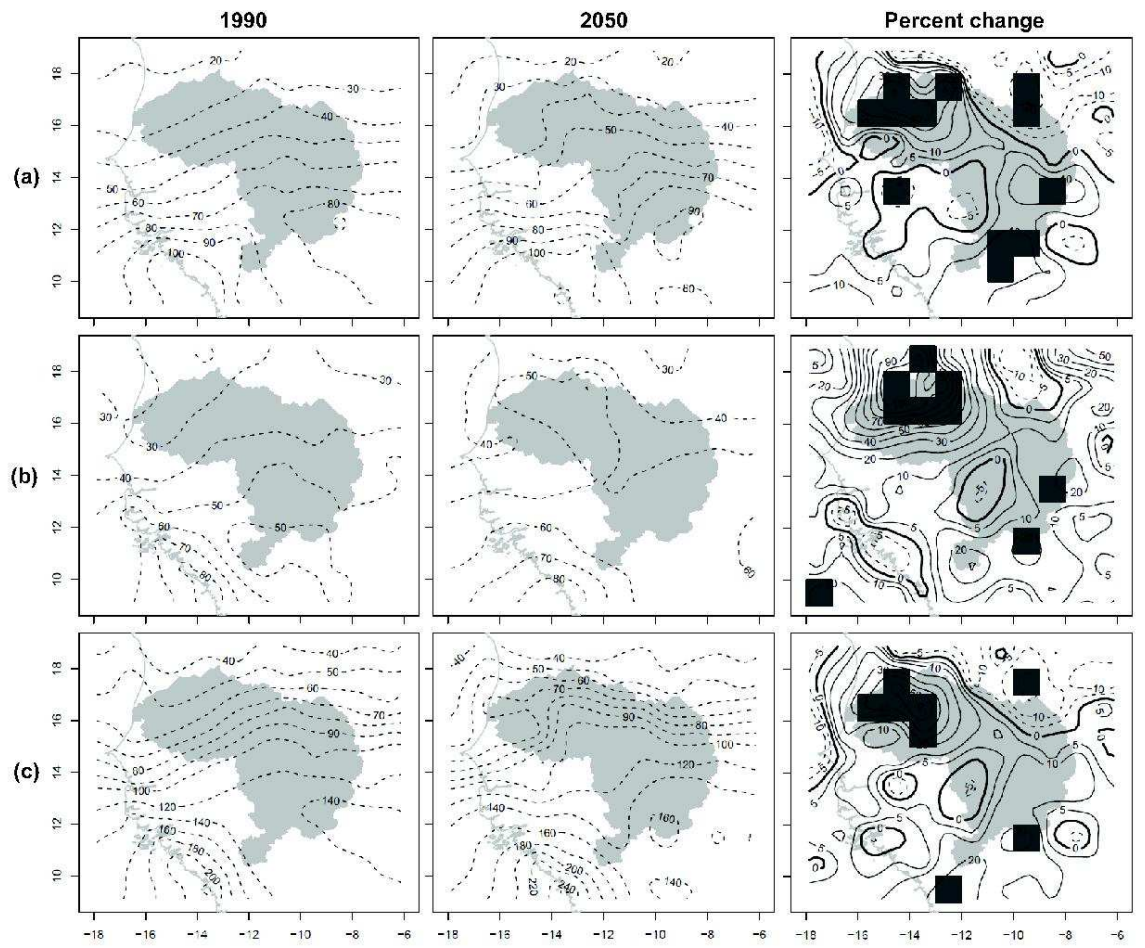

Fig. 4 Interpolated maps from the GAMLSS analysis for each site: (a) mean $\mu$, (b) standard deviation $\sigma$, and (c) associated with $T r=10$ years. The maps for the years 1990 and 2050 , and the percentage difference between them, are presented. In the difference maps, negative values are dashed, while the confidence in projected changes is highlighted with the dark gray square.

upper basin,while in the Sahelian zone a significant decrease is projected. In the Valley area, the expected increase in $\mu$ will be about $10 \mathrm{~mm}$, very similar to the increment for the upper basin, however in the lower basin represents an increase of over $40 \%$. The maps of $\sigma$ (Fig. 4(b)) retain the latitudinal gradient of rainfall maps, although less significant than the $\mu$ maps. The difference between the two maps shows that in the lower basin a significant increase in $\sigma$ is projected, which could reach $100 \%(30 \mathrm{~mm})$, while in the upper basin the projected increase in this parameter hardly exceeds $20 \%(10 \mathrm{~mm})$. Finally, the maps for $T r=10$ years (Fig. 4(c)) show a clear latitudinal gradient, with a decrease in maximum annual rainfall northwards. A general increase in the AMDR associated with $T r=10$ years is expected, except at the northern edge of the basin and in some isolated areas of the study area within the Sudanian zone. This significant increase is foreseen in the lower basin, where a difference of more than $20-30 \mathrm{~mm}$ was calculated between the reference years $(40-60 \%)$.

\section{CONCLUSIONS}

In order to distinguish the predicted changes in the severity and frequency of AMDR in the Senegal River Basin, plausible climate scenarios provided by RCMs and observed data were considered. These databases, together with the GAMLSS methodology, allow consideration of the 
non-stationarity present in hydrometeorology in the Senegal River Basin. However, the good fit of the GAMLSS statistical model to simulated AMDR time series does not imply a proper coupling to the observed series from IRD database. Considering the observed grids of rainfall data, a bias analysis of AMDR time series was obtained from the RCM that allowed evaluation of the ability of climate models. Since an "ideal model" was not identified for the study area, in order to exploit all the information provided by the RCMs, an ensemble pdf was constructed for each site based on the bias analysis. Finally, the ensemble pdf which has been built for each site, allows estimation of the spatio-temporal change of AMDR in the study area. From the interpolated maps, various parameters of the ensemble pdf maintain the latitudinal gradient of rainfall in the area. However, in the difference maps for the reference years considered (1990 and 2050); this spatial pattern is not preserved. The percentage difference shows that in the Valley area, the increase of AMDR will be significantly higher than in the upper watershed area. In the Valley area, the increase in $\mu$ is projected to be more than $40 \%$ and the increase in $\sigma$ to be close to $100 \%$. Both increases will be reflected in the maximum precipitation amount for the $90 \%$ quantile, which will rise by $40-60 \%$ in the lower part of the basin.

Due to the high vulnerability of the population of West Africa to climate variability and change, plausible AMDR maps could be used by stakeholders to develop mitigation and adaptation strategies, and to build "adaptive capacity".

Acknowledgements The authors acknowledge the support from EU Project AMMA (FP6004089), and from R\&D Project CGL2008-02530/BTE financed by the Spanish Ministry of Science and Innovation.

\section{REFERENCES}

Andersen, J., Refsgaard, J. \& Jensen, K. H. (2001) Distributed hydrological modelling of the Senegal River basin - mode construction and validation. J. Hydrol. 247, 200-214.

Christensen, J. H., Rummukainen, M. \& Lenderink, G. (2009) Formulation of very-high-resolution regional climate model ensembles for Europe. In: ENSEMBLES: Climate change and its impacts at seasonal, decadal and centennial timescales. Summary of research and results from the ENSEMBLES project (ed. by P. van der Linden \& J. F. B. Mitchell), 47-58. Met Office Hadley Centre, FitzRoy Road, Exeter EX1 3PB, UK.

Diedhiou, A., Janicot, S., Viltard, A., de Félice.P. \& Laurent, H. (1999) Easterly waves regimes and associated convection over , B. \& Tibshirani, R. J. (1993) An Introduction to the Bootstrap. Chapman \& Hall, New York, NY, USA.

García, S. G. \& Giraldo, J. D (2010) Analysis of impacts on hydrometeorological extremes in the Senegal River Basin from REMO S. G. \& Giraldo, J. D. (2010) Analysi

Huntington, T. G. (2006) Evidence for intensification of the global water cycle: review and synthesis. J. Hydrol. 319, 83-95.

Janicot, S. \& Sultan, B. (2001) Intra-seasonal modulation of convection in the West African monsoon. Geophys. Res. Lett.. 28 $523-526$.

Karambiri, H., García, S. G., Giraldo, J. D., Yacouba, H., Ibrahim, B., Barbier, B. et al. (2011) Assessing the impact of climate variability and climate change on runoff in West Africa: the case of Senegal and Nakambe river basins. Atmos. Sci. Lett. 12(1), 109-115.

Kundzewicz, Z. W., Mata, L. J., Amell, N. W., Döll, P., Kabat, P., Jiménez, B., et al. (2007) Freshwater resources and their management. In: Climate Change 2007: Impacts, Adaptation and Vulnerability. Contribution of Working Group II to the Palutikof, P. J. van der Linden \& C. E. Hanson), 173-210. Cambridge University Press, Cambridge, UK.

Lebel, T., Cappelaere, C., Galle, S., Hanan, N., Kergoat, L., Levis, S., Vieux, B., Descroix, L., Gosset, M., Mougin, E., Peugeot, C. \&

Marques de Sa, J. P. (2007) Applied Statistics using SPSS, STATISTICA, and MATLAB, 2nd ed. Springer-Verlag, Berlin Germany.

Messager, G., Gallée, H. \& Brasseur, O. (2004) Precipitation sensitivity to regional SST in a regional climate simulation during the West African monsoon for two dry years. Clim. Dyn. 22, 249-266.

Milly, P. C. D., Betancourt, J., Falkenmark, M., Hirsch, R. M., Kundzewicz, Z. W., Lettenmaier, D. P., et al. (2008) Stationarity is dead: whiter water management? Science 319, 573-574.

Olson, D. M., Dinerstein, E., Wikramanayake, E. D., Burgess, N. D., Powell, G. V. N., Underwood, E. C., D’Aamico, J. A., Itoua, I., Strand, H. E., Morrison, J. C., Loucks, C. J., Allnutt, T. F., Ricketts, T. H., Kura, Y., Lamoreux, J. F., Wettengel, W. W., Hedao, P. \& Kassem, K.R. (2001) Terrestrial ecoregions of the world: a new map of life on Earth. BioScience 51(11), 933-938.

Paeth, H., Bom, K., Podzun, R. \& Jacob, D. (2005) Regional dynamical downscaling over West Africa: model evaluation and comparison of wet and dry years. Met. Z. 14 (3),349-367.

Rigby, R. A. \& Stasinopoulos, D. M. (2005) Generalized additive models for location scale and shape. Appl. Stat. 54, 507-554. 
Sheskin, D.J. (2000) Handbook of Parametric and Nonparametric Statistical Procedures, 2nd ed. Chapman \& Hall/CRC, Boca Raton, Florida, USA. Stasinopoulos D. M. \& Rigby R. A. (2007) Generalized additive models for location scale and shape (GAMLSS) in R. J. Stat.
Software 23 (7), 1-46.

van Buuren, S. \& Fredriks, M. (2001) Worm plot: a simple diagnostic device for modelling growth reference curves. Statist. Med. 20, 1259-1277.

Villarini, G., Smith, J. A., Serinaldi, F., Bales, J., Bates, P. D. \& Krajewski, W. F. (2009) Flood frequency analysis for nonstationary annual peak records in an urban drainage basin. Adv. Water Res. 32(8), 1255-1266.

Villarini, G., Smith, J. A. \& Napolitano, F., (2010) Nonstationary modeling of a long record of rainfall and temperature over Rome, 



\section{REGISTROS DE PROPIEDAD INTELECTUAL}

\subsection{Registro de Propiedad Intelectual 1 (Subpixels Toolbox)}

\subsubsection{Aportación al trabajo realizado}

Con el objetivo de simplificar la ejecución de los procedimientos involucrados en el Análisis de Sub-píxeles (ver Figura 9), diferentes comandos se desarrollaron en el SIG GRASS: primero, el comando i.subpixel ejecuta el Modelo de Mezcla Lineal (LMM) y el Análisis de Coherencia Espacial (SCA); luego, el comando i.dod lleva a cabo el análisis de las trayectorias de drenaje, extraídas de la topografía digital, con el algoritmo de Drenaje basado en DEM (DOD); finalmente, el comando i.isolated reclasifica los clúster espurios de pocos píxeles con el algoritmo de Identificación de Clúster Aislados (ICI). Debe resaltarse que la herramienta fue desarrollada con lenguajes de código fuente abierto, bajo licencia GNU-GPL, lo que promoverá su utilización en países en vías de desarrollo. Constituye un claro aporte al desarrollo de estrategias de adaptación al cambio y variabilidad climática frente a inundaciones.

El siguiente artículo, que hace parte de este compendio de publicaciones, presenta un buen ejemplo de aplicación del Toolbox Subpixel para la detección de zonas inundadas:

- Giraldo Osorio, J. D, García Galiano, S. G., 2012. Development of a sub-pixel analysis method applied to dynamic monitoring of floods, International Journal of Remote Sensing, 33 (7), 2277-2295. 



\subsubsection{Copia del registro}

\section{REGISTRO GENERAL DE LA PROPIEDAD INTELECTUAL}

Según lo dispuesto en la Ley de Propiedad Intelectual (Real Decreto Legislativo 1/1996, de 12 de abril), quedan inscritos en este Registro los derechos de propicdad intelectual en la forma que se determina seguidamente:

\section{NÚMERO DE ASIENTO REGISTRAL 08 / 2011/ 173}

Título: Subpixels Toolbox

Objeto de propiedad intelectual: Programa de ordenador Clase de obra: Programa de ordenador

PRIMERA INSCRIPCIÓN

\section{Autor/es y titular/es originarios de derechos}

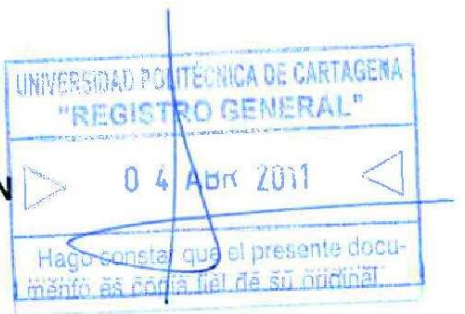

- Apellidos y nombre: GARCÍA GALIANO, Sandra Gabriela

Nacionalidad: ESP

D.N.I./N.I.F./Pasaporte: 48610639-R

Parte de la obra de la que es autor: $30 \%$

- Apellidos y nombre: GIRALDO OSORJO, Juan Diego

Nacionalidad: COL

D.N.I./N.I.F./Pasaporte: X7336072-S

Parte de la obra de la que es autor: $70 \%$

\section{Transmisión de derechos}

- Titular cesionario: UNIVERSIDAD POLITECNICA DE CARTAGENA

Nacionalidad: ESP

D.N.I.N.I.F./Pasaporte: Q8050013E

Es titular en exclusiva de los derechos de explotación de esta obra por mediar relación laboral y estar creada en el ejercicio de las funciones asignadas en la empresa al autor/es.

- Cedente: GARCÍA GALIANO, Sandra Gabriela

Nacionalidad: ESP

D.N.I./N.I.F./Pasaporte: 48610639-R

- Cedente: GIRALDO OSORIO, Juan Diego

Nacionalidad: COL

D.N.I./N.I.F./Pasaporte: X7336072-S

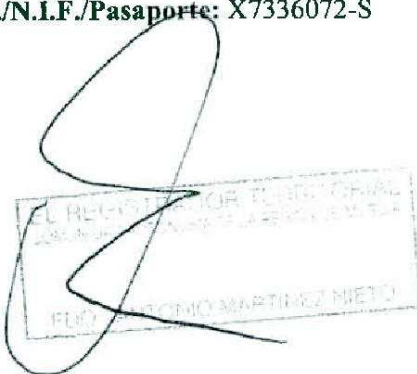


Datos de la solicitud

Núm. solicitud: MU-163-2011

Fecha de presentación y efectos: 23/02/2011 Hora: 11:43

En Murcia, a once de marzo de dos mil once
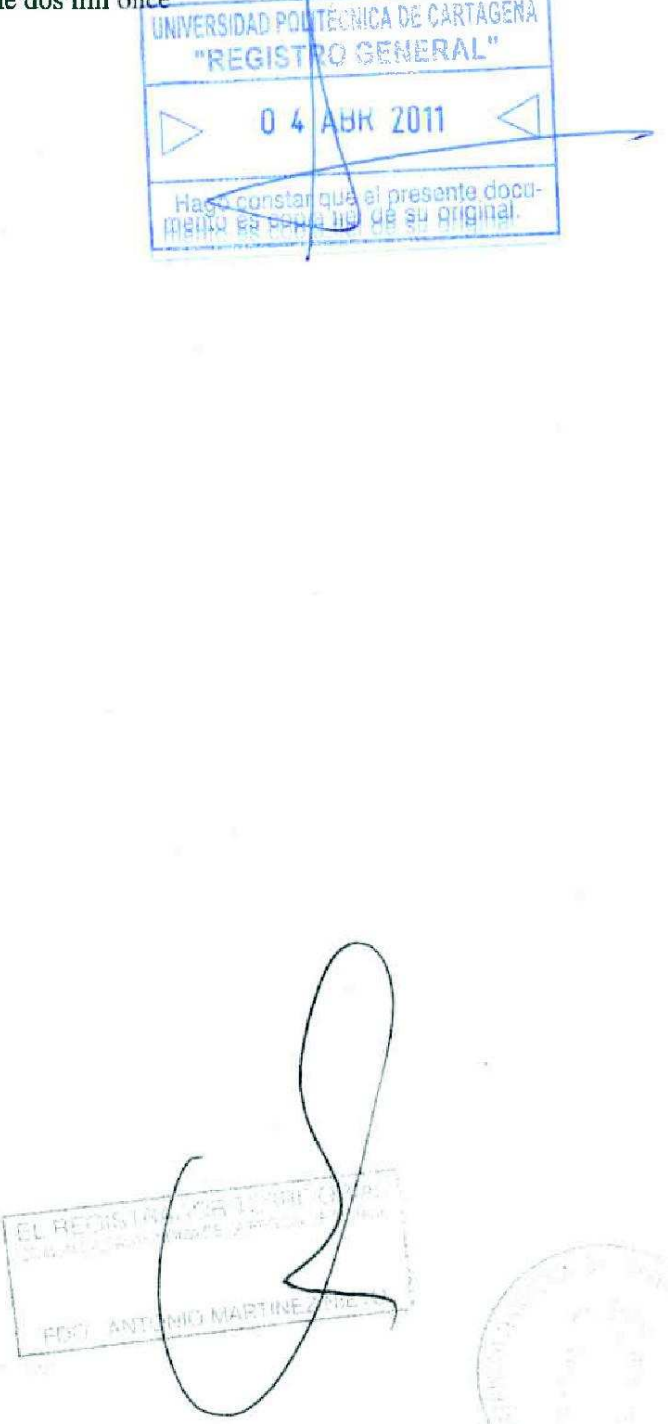


\subsection{Registro de Propiedad Intelectual 2 (Spells Toolbox)}

\subsubsection{Aportación al trabajo realizado}

Dos comandos, desarrollados bajo el SIG GRASS, facilitan el análisis de rachas con series de mapas (r.series.spell y r.series.spell.point). El comando r.series.spell permite construir mapas que muestran la distribución espacial de diferentes estadísticos de las rachas. Así por ejemplo, es posible analizar las rachas correspondientes al número de días consecutivos con temperaturas superiores a los $30 \stackrel{\circ}{ } \mathrm{C}$, y construir el mapa con la longitud media de las rachas de temperaturas altas, del número de días que superan la temperatura umbral para cada año, etc. Por otra parte, el comando r.series.spell.point permite obtener la serie temporal de la racha para un punto determinado, de manera que se pueden ejecutar análisis sobre la distribución de probabilidad de las rachas en un sitio o localización determinado.

Es importante resaltar que los comandos han sido codificados utilizando lenguajes de programación y SIG con licencias GNU-GPL, posibilitando que la metodología de análisis sea aplicable por los gestores y responsables de las decisiones en países en vías de desarrollo para la definición de estrategias de adaptación al cambio y variabilidad climática frente a eventos extremos (sequías, rachas húmedas, rachas de temperaturas extremas, etc.)

Se debe destacar que el software Spells Toolbox, se encuentra entre la transferencia tecnológica de la UPCT a la Spin Off FutureWater S.L. en fase de constitución (aprobada en Reunión de Consejo de Gobierno del 22 de diciembre de 2011).

Spells Toolbox fue utilizado en los siguientes artículos:

- García Galiano, S. G. and Giraldo Osorio, J. D., 2010. Analysis of impacts on hydrometeorological extremes in the Senegal river basin from REMO RCM. Meteorologische Zeitschrift, 19 (4), 375-384.

- Karambiri, H., García Galiano, S. G., Giraldo, J. D., Yacouba, H., Ibrahim, B., Barbier, B. Polcher, J., 2011. Assessing the impact of climate variability and climate change on runoff in West Africa: the case of Senegal and Nakambe River basins, Atmospheric Science Letters, AMMA Special Issue, 12: 109-115. 



\subsubsection{Copia del registro}

\section{REGISTRO GENERAL DE LA PROPIEDAD INTELECTUAL}

Según lo dispuesto en la Ley de Propiedad Intelectual (Real Decreto Legislativo 1/1996, de 12 de abril), quedan inscritos en este Registro los derechos de propiedad intelectual en la forma que se determina seguidamente:

\section{NÚMERO DE ASIENTO REGISTRAL 08 / 2011 / 176}

Título: Spells Toolbox

Objeto de propiedad intelectual: Programa de ordenador Clase de obra: Programa de ordenador

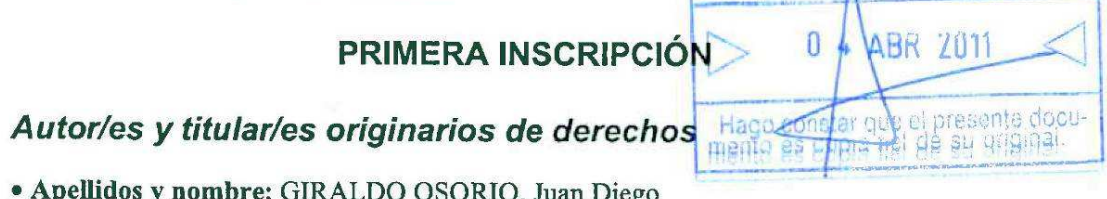

- Apellidos y nombre: GIRALDO OSORIO, Juan Diego

Nacionalidad: COL

D.N.I./N.I.F./Pasaporte: X7336072-S

Parte de la obra de la que es autor: $70 \%$

- Apellidos y nombre: GARCÍA GALIANO, Sandra Gabriela

Nacionalidad: ESP

D.N.I./N.I.F./Pasaporte: $48610639-$ R

Parte de la obra de la que es autor: $30 \%$

\section{Transmisión de derechos}

- Titular cesionario: UNIVERSIDAD POLITECNICA DE CARTAGENA Nacionalidad: ESP

D.N.I./N.I.F./Pasaporte: Q8050013E

Es titular en exclusiva de los derechos de explotación de esta obra por mediar relación laboral y estar creada en el ejercicio de las funciones asignadas en la empresa al autor/es.

- Cedente: GIRALDO OSORIO, Juan Diego

Nacionalidad: $\mathrm{COL}$

D.N.I./N.I.F./Pasaporte: X7336072-S

- Cedente: GARCÍA GALIANO, Sandra Gabriela

Nacionalidad: ESP

D.N.I./N.I.F./Pasaporte: 48610639. R

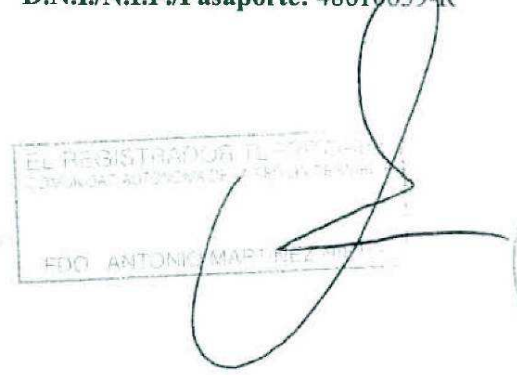




\section{Datos de la solicitud}

Núm. solicitud: MU-166-2011

Fecha de presentación y efectos: 24/02/2011 Hora: 09:27

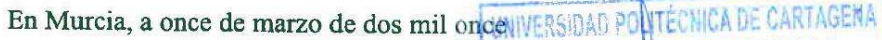
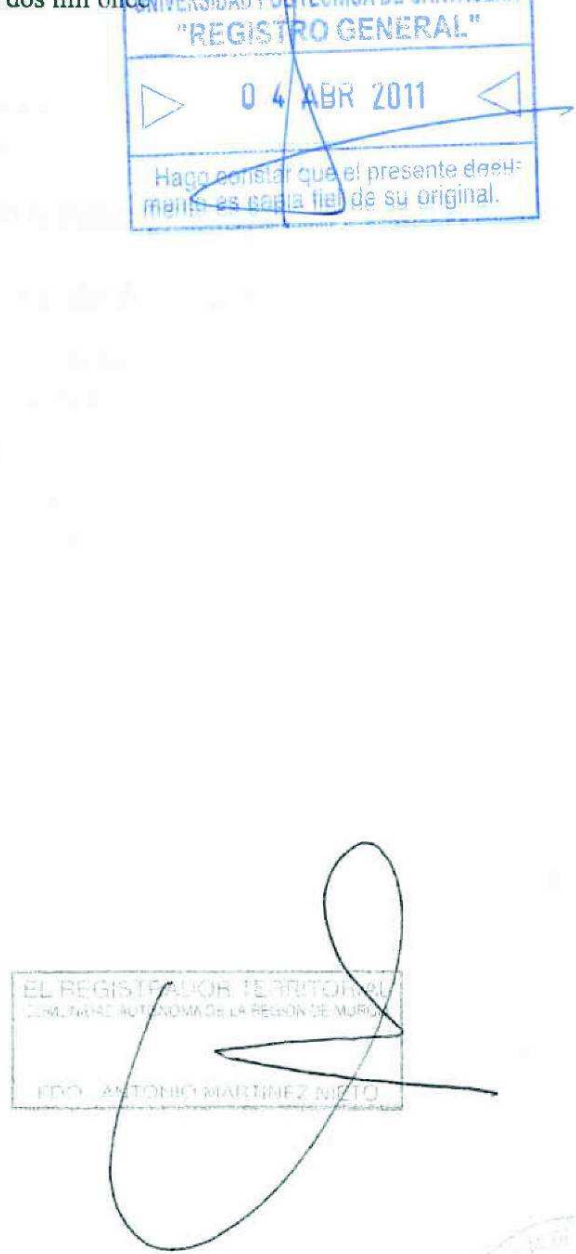


\subsection{Registro de Propiedad Intelectual 3 (Series Toolbox)}

\subsubsection{Aportación al trabajo realizado}

Dos comandos, desarrollados bajo el SIG GRASS, facilitan el análisis de series de mapas (r.series.new y r.series.new.point). El comando r.series.new permite construir con facilidad distribuciones espaciales de los diferentes estadísticos de la serie de mapas (media, desviación estándar, valor máximo o mínimo, etc.). Es posible analizar gran cantidad de datos para obtener estas distribuciones. El comando ha sido utilizado con mapas de temperatura y precipitación diaria obtenidos desde RCMs, los cuales pueden tener hasta 150 años (más de 50000 mallas de datos). Por otra parte, el comando r.series.point permite obtener los datos de la serie de mapas para un punto determinado, de manera que se pueden ejecutar análisis de la serie temporal de los datos (análisis de tendencia, distribución de probabilidad de los datos, etc.).

Es importante resaltara que los comandos han sido codificados utilizando lenguajes de programación y SIG con licencias GNU-GPL, posibilitando que la metodología de análisis sea aplicable por los gestores y responsables de la toma de decisiones en países en vías de desarrollo.

Se debe destacar que el software Spells Toolbox, se encuentra entre la transferencia tecnológica de la UPCT a la Spin Off FutureWater S.L. en fase de constitución (aprobada en Reunión de Consejo de Gobierno del 22 de diciembre de 2011).

Series Toolbox fue utilizado en los siguientes artículos:

- Giraldo Osorio, J. D. and García Galiano, S. G., 2011. Building hazard maps of extreme daily rainy events from PDF ensemble, via REA method, on Senegal River Basin. Hydrol. Earth Syst. Sci., 15, 3605-3615.

- Garcia Galiano, S. G., Giraldo Osorio, J. D., 2011. Non stationary analysis of spatial patterns of extreme rainfall events in West Africa. In: Hydro-climatology: variability and change. (Eds. S.W. Franks, E. Boegh, E. Blyth, D.M. Hannah, K.K. Yilmaz). IAHS Publ. 344, IAHS Press, Wallingford, Oxfordshire, United Kingdom. 



\subsubsection{Copia del registro}

\section{REGISTRO GENERAL DE LA PROPIEDAD INTELECTUAL}

Según lo dispuesto en la Ley de Propiedad Intelectual (Real Decreto Legislativo 1/1996, de 12 de abril), quedan inscritos en este Registro los derechos de propiedad intelectual en la forma que se determina seguidamente:

\section{NÚMERO DE ASIENTO REGISTRAL 08 / 2011 / 177}

Título: Series Toolbox

Objeto de propiedad intelectual: Programa de ordenador Clase de obra: Programa de ordenador

PRIMERA INSCRIPCIÓN

Autor/es y titular/es originarios de derechos

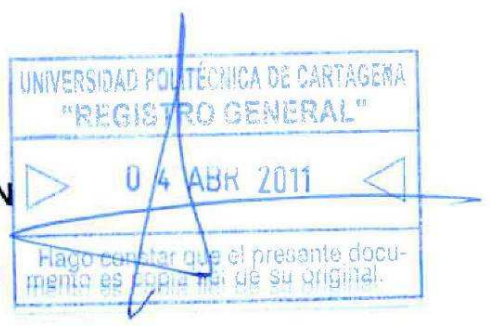

- Apellidos y nombre: GIRALDO OSORIO, Juan Diego Nacionalidad: $\mathrm{COL}$

D.N.I./N.I.F./Pasaporte: X7336072-S

Parte de la obra de la que es autor: $70 \%$

- Apellidos y nombre: GARCÍA GALIANO, Sandra Gabriela

Nacionalidad: ESP

D.N.I./N.I.F./Pasaporte: $48610639-$ R

Parte de la obra de la que es autor: $30 \%$

\section{Transmisión de derechos}

- Titular cesionario: UNIVERSIDAD POLITECNICA DE CARTAGENA

Nacionalidad: ESP

D.N.I./N.I.F./Pasaporte: Q8050013E

Es titular en exclusiva de los derechos de explotación de esta obra por mediar relación laboral y estar creada en el ejercicio de las funciones asignadas en la empresa al autor/es.

- Cedente: GIRALDO OSORIO, Juan Diego Nacionalidad: COL

D.N.I./N.I.F./Pasaporte: X7336072-S

- Cedente: GARCÍA GALIANO, Sandra Gabriela

Nacionalidad: ESP

D.N.I./N.I.F./Pasaporte: $48610639-R$ 


\section{Datos de la solicitud}

Núm. solicitud: MU-167-2011

Fecha de presentación y efectos: 24/02/2011 Hora: 09:31

En Murcia, a once de marzo de dos mil once
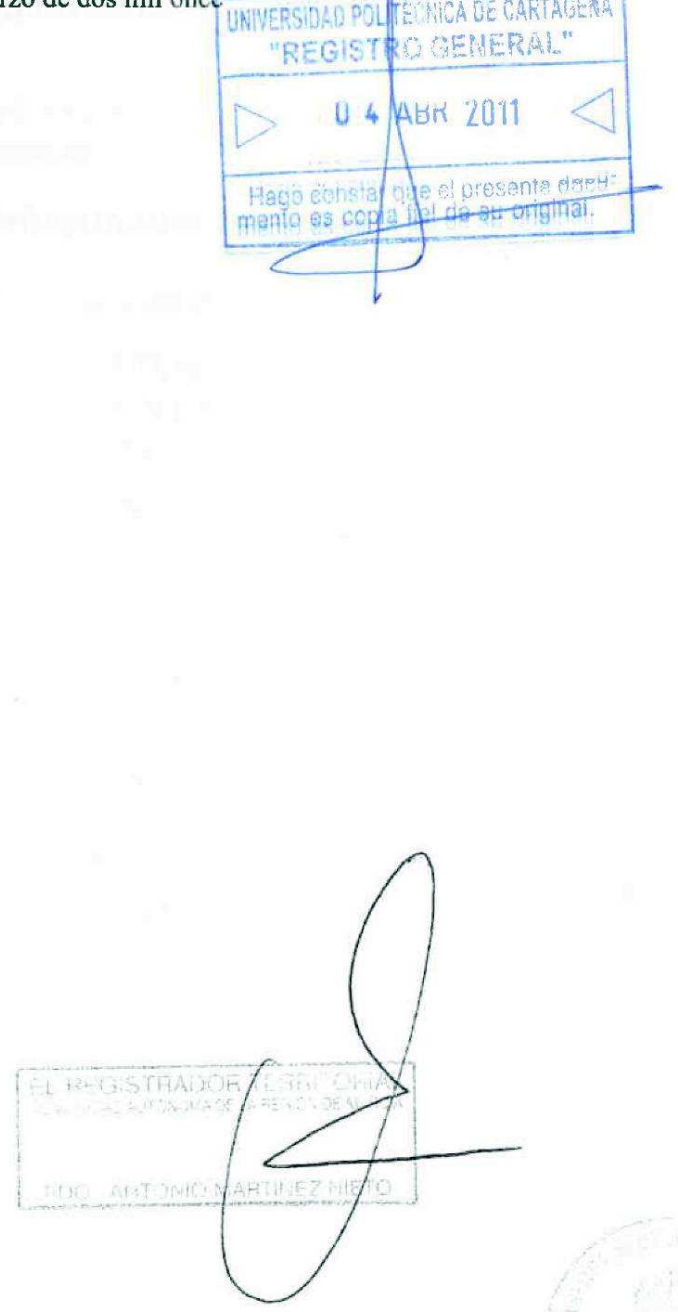


\section{RESUMEN DE LOS RESULTADOS Y CONCLUSIONES}

Con el objetivo de distinguir los cambios predichos en la severidad y frecuencia de los eventos extremos (precipitaciones máximas y rachas de sequía), se ha trabajado con datos provistos por RCMs. Esta base de datos, junto con la metodología GAMLSS, permiten tener en consideración la no estacionariedad hidroclimática que presenta la Cuenca del Río Senegal. Sin embargo, el buen ajuste del modelo estadístico GAMLSS a las series temporales hidrológicas, no implica un acople apropiado a la serie observada. Considerando mallas de datos de precipitación observada, se efectuó un análisis del desvío de las variables hidrológicas consideradas obtenidas desde los RCMs, que permitió evaluar el desempeño de los modelos climáticos. Se concluye que no existe un RCM que sea mejor para representar la Longitud Máxima Anual de las Rachas Secas (Annual Maximum Dry Spell Length, AMDSL) o la Precipitación Diaria Máxima Anual (Annual Maximum Daily Rainfall, AMDR) en toda el área de estudio considerada. Para las AMDSL, los análisis fueron regionales, ya que las sequías son un fenómeno que afecta grandes extensiones, a diferencia de los eventos de tormenta, que son bastante localizados. Por esta razón, se optó por construir para las AMDR una PDF ensemble en cada sitio de análisis ( grid-site, o celda de la malla), que tuviera en cuenta el resultado del análisis de desvío y de convergencia. Finalmente, para las AMDR, la PDF ensemble construida en cada sitio permitió calcular diferentes estadísticos de interés, que fueron interpolados para diferentes años, obteniéndose la evolución espacio-temporal de las AMDR en la zona de estudio. Para las AMDSL, como ya se ha dicho, se han alcanzado conclusiones a nivel de ecorregión.

En conclusión, una novedosa aproximación con PDF ensemble no estacionarias ha sido desarrollada en esta tesis, que ha permitido la identificación de tendencias espacio-temporales plausibles, en el caso de la distribución de las AMDR. Desde los datos de los RCM, se ha demostrado que el calentamiento global afectará la frecuencia e intensidad de los eventos de precipitación extrema en la Cuenca del Río Senegal. El impacto sobre la sociedad dependerá no sólo de la intensidad del evento, sino también de la habilidad de la población para adaptarse a las nuevas condiciones. El conocimiento y entendimiento para adoptar las medidas adecuadas es importante.

De acuerdo con García Galiano and Giraldo Osorio (2010), las proyecciones del RCM REMO predicen incrementos plausibles de los cuantiles más altos de la distribución de las Longitudes de Racha Seca (Dry Spell Length, DSL), para el umbral de precipitación $1 \mathrm{~mm} /$ día, especialmente en la zona del Sahel para el periodo 2021-2050. Sin embargo, el aumento no es significativo para el periodo 1991-2020. Para el umbral 10 
mm/día el aumento en los cuantiles más altos de la distribución será significativo para toda la cuenca en el periodo 2021-2050, sin embargo no será significativo en 19912020.

En el trabajo Karambiri et al. (2011) se demostró que para 2050, se proyecta un decrecimiento de la precipitación en las ecorregiones estudiadas al interior de la Cuenca del Río Senegal (SAS y WSS), de acuerdo con los RCM KNMI/RACMO y MPI/REMO, excepto en la Cuenca del Nakambé, donde MPI/REMO predice un aumento de la precipitación. Por otra parte, las AMDSL presentan una tendencia creciente de acuerdo con estos RCM, especialmente en la región Sudaniana de ambas cuencas. Sin embargo, a pesar de la tendencia decreciente de la precipitación, el análisis de impacto con el modelo hidrológico en la Cuenca del Río Nakambé demostró una tendencia creciente de los caudales, revelando la importancia de considerar la variación del uso del suelo al simular el ciclo hidrológico. El desarrollo de escenarios de cambio en el uso del suelo, es necesario para desarrollar herramientas que apoyen efectivamente las decisiones que tienen como objeto una mejor adaptación al cambio climático en el Sahel.

El los artículos García Galiano and Giraldo Osorio (2011), y Giraldo Osorio and García Galiano (2011) se determinó que los mapas interpolados para los diferentes parámetros de la PDF ensemble de las AMDR conservan, en mayor o menor medida, el característico gradiente latitudinal de la precipitación. Sin embargo, en el mapa de diferencias para los años de referencia considerados (1990-2050), este patrón espacial parece no existir. La diferencia porcentual muestra que en el área del valle, el aumento de las AMDR será significativamente mayor que en la zona alta de la cuenca, donde aún pueden presentarse algunas pequeñas zonas con valores negativos. En la zona del Valle del Río Senegal, se proyecta un aumento significativo de la media (> $40 \%$ ), y de la desviación estándar ( 100\%), que se verá reflejado en el aumento considerable del cuantil $90 \%$ de la distribución de precipitación máxima en la zona baja de la cuenca (entre 40-60\%).

La evidencia presentada por los anteriores trabajos reveló la necesidad de desarrollar una herramienta de libre acceso, potente y de fácil ejecución para la detección y seguimiento de inundaciones en el Valle del Río Senegal. El balance con la resolución temporal para el seguimiento de las inundaciones, así como la necesidad de incorporar imágenes de sensores de libre acceso, restringió la resolución espacial de las imágenes. Sin embargo, se desarrolló una metodología de Análisis de Sub-píxeles (SA), que presenta como ventaja su aplicación directa en el seguimiento de eventos altamente dinámicos, como son las inundaciones, utilizando imágenes de satélite de 
moderada resolución espacial y con alta resolución temporal. Además, se utiliza el DEM subyacente de la zona de estudio para mejorar la identificación de las zonas inundadas. Luego, al basarse en información libremente accesible, su aplicación por parte de responsables de la toma de decisión en países en vías de desarrollo es potenciada.

Los resultados de la clasificación SA fueron evaluadas tomando como referencia el mapa clasificado Landsat, con resolución espacial modificada a $50 \mathrm{~m}$. La clasificación fue validada en varias zonas del Valle del Río Senegal, que presentaban áreas inundadas con diferentes características. En todas ellas la metodología de SA mejoró considerablemente la identificación de la inundación, respecto de la clasificación supervisada tradicional. Considerando toda el área de estudio, el método SA identificó correctamente el $80 \%$ de las áreas realmente inundadas, mientras que el método tradicional sólo identificó correctamente el 39\%.

Otro claro aporte del presente trabajo a la construcción de capacidad adaptativa, son los mapas de riesgo de eventos de precipitación extrema. Aprovechando herramientas de análisis de última generación (los diferentes modelos climáticos, la posibilidad de ejecutar análisis no estacionarios, bootstrapping), se construyeron PDF ensemble para los grid-sites (puntos sobre la grilla) en África Occidental. De estas PDF ensemble puntuales se calcularon estadísticos básicos (media y desviación estándar) y diferentes cuantiles (entre 5-95\%) asociados con varios periodos de retorno, junto con sus correspondientes intervalos de confianza. Dada la naturaleza no estacionaria de las PDF ensemble, estos estadísticos fueron calculados en varios años, y en dos artículos que componen la Tesis (Giraldo and García, 2011; García and Giraldo, 2011) se calcularon las diferencias entre el mapa para el año de referencia 1990, y el construido para el horizonte de pronóstico 2050. Además de la magnitud de la diferencia, los mapas presentan las zonas donde ésta será significativa. Se puede concluir para cualquier punto en la zona de estudio si, a futuro, los eventos de tormenta más virulentos se harán más frecuentes, o si por el contrario serán necesarias nuevas medidas que protejan a la población y las infraestructuras que allí se asientan.

Las líneas de investigación futuras deberían estar encaminadas a analizar más profundamente otros métodos para ponderar los modelos miembros en la PDF ensemble, considerando su aplicación para construir los patrones espaciales de otras características de la precipitación diaria (longitud máxima anual de las rachas secas, número de días sin lluvias a nivel anual o estacional, etc) $u$ otras variables hidroclimáticas (evaporación, temperatura, precipitación mensual, etc) con 
implicaciones en la gestión de los recursos hídricos a escala de cuenca. La metodología aplicada en esta Tesis debe ser comparada con otras propuestas para calcular y relacionar los factores de ponderación, con el fin de verificar la consistencia temporal de este procedimiento. 


\section{REFERENCIAS}

Andersen, J., Refsgaard, J. and Jensen, K. H., 2001. Distributed hydrological modelling of the Senegal river basin - model construction and validation. J. Hydrol., 247, 200-214.

Benger, S., 2003. Remotely sensed determination of flood surface gradients for hydrological modelling of semi-arid floodplains. In Proceedings of Geoscience and Remote Sensing Symposium. IEEE International, 4, 2950-2952.

Blenkinsop, S. and Fowler, H. J., 2007. Changes in drought frequency, severity and duration for the British Isles projected by the PRUDENCE regional climate models. J. Hydrol., 342, 50-71.

Brivio, P., Colombo, R., Maggi, M. and Tomasoni, R., 2002. Integration of remote sensing data and GIS for accurate mapping of flooded areas. Int. J. Remote Sens., 23, 429-441.

Buser, C. M., Künsch, H. R., Lüthi, D., Wild, M. and Schär C., 2009. Bayesian multimodel projection of climate: bias assumptions and interannual variability. Clim. Dynam., 33, 849-868, doi: 10.1007/s00382-009-0588-6.

Christensen, J. H., Rummukainen, M., and Lenderink, G., 2009. Formulation of veryhigh-resolution regional climate model ensembles for Europe. In ENSEMBLES: Climate change and its impacts at seasonal, decadal and centennial timescales: Summary of research and results from the ENSEMBLES project, edited by: van der Linden, P. and Mitchell, J. F. B., Met Office Hadley Centre, FitzRoy Road, Exeter EX1 3PB, UK, 47-58.

Cour, J. M., 2001. The Sahel in West Africa: countries in transition to a full market economy. Global Environ. Change, 11, 31-47.

Dee, D. P., Uppala, S. M., Simmons, A. J., Berrisford, P., Poli, P., Kobayashi, S., Andrae, U., Balmaseda, M. A., Balsamo, G., Bauer, P., Bechtold, P., Beljaars, A. C. M., van de Berg, L., Bidlot, J., Bormann, N., Delsol, C., Dragani, R., Fuentes, M., Geer, A. J., Haimberger, L., Healy, S. B., Hersbach, H., Hólm, E. V., Isaksen, L., Kållberg, P., Köhler, M., Matricardi, M., McNally, A. P., MongeSanz, B. M., Morcrette, J.-J., Park, B.-K., Peubey, C., de Rosnay, P., Tavolato, C., Thépaut, J.-N. and Vitart, F., 2011. The ERA-Interim reanalysis: configuration and performance of the data assimilation system. $Q$. J. $R$. Meteorol. Soc., 137, 553-597. 
Descroix, L., Mahé, G., Lebel, T., Favreau, G., Galle, S., Gautier, E., Olivry, J. C., Albergel, J., Amogu, O., Cappelaere, B., Dessouassi, R., Diedhiou, A., Le Breton, E., Mamadou, I. and Sighomnou, D., 2009. Spatiotemporal variability of hydrological regimes around the boundaries between Sahelian and Sudanian areas of West Africa: a synthesis. J. Hydrol., 375, 90-102.

Dettinger, M., 2005. From climate-change spaghetti to climate-change distributions for 21st century California. San Francisco Estuary Watershed Sci., 3 (1), article 4.

Diedhiou, A., Janicot, S., Viltard, A., De Félice, P. and Laurent, H., 1999. Easterly wave regimes and associated convection over West Africa and tropical Atlantic: results from the NCEP/NCAR and ECMWF reanalyses. Clim. Dyn., 15, 795822.

European Commission, 2009. Technical report-2009-040. Common implementation strategy for the water framework directive (2000/60/EC). Guidance document No. 24. River basin management in a changing climate. European Comunities, 134 pp. http://ec.europa.eu/environment/water/water-framework/

Efron, B. and Tibshirani, R. J., 1993. An introduction to the bootstrap. Chapman \& Hall, New York, NY, USA.

Farr, T. G., Rosen, P. A., Caro, E., Crippen, R., Duren, R., Hensley, S., Kobrick, M., Paller, M., Rodríguez, E., Roth, L., Seal, D., Shaffer, S., Shimada, J., Umland, J., Werner, M., Oskin, M., Burbank, D. and Alsdorf, D., 2007. The shuttle radar topography mission. Rev. Geophys., 45, RG2004, doi: 10.1029/2005RG000183.

Favreau, G., Cappelaere, B., Massuel, S., Leblanc, M., Boucher, M., Boulain, N. and Leduc, C., 2009. Land clearing, climate variability, and water resources increase in semiarid southwest Niger: a review. Water Resour. Res., 45: W00A16.

Filliben, J. J., 1975. The probability plot correlation coefficient test for normality. Technometrics, 17 (1), 111-117.

Fontaine, B., Roucou, P. and Monerie, P. A., 2011. Changes in the African monsoon region at medium-term time horizon using 12 AR4 coupled models under the A1b emissions scenario. Atmos.Sci. Lett., 12, 83-88, doi: 10.1002/asl.321.

Fox, J., 2002. Bootstrapping regression models. Appendix to An R and S-PLUS companion to applied regression. 
García Galiano, S. G. and Giraldo Osorio, J. D., 2010. Analysis of impacts on hydrometeorological extremes in the Senegal river basin from REMO RCM. Meteorol. Z., 19 (4), 375-384.

Garcia Galiano, S. G., Giraldo Osorio, J. D., 2011. Non stationary analysis of spatial patterns of extreme rainfall events in West Africa, In: Hydro-climatology: variability and change (Eds. S.W. Franks, E. Boegh, E. Blyth, D.M. Hannah, K.K. Yilmaz). IAHS-AISH Publication, 344, 75-81. IAHS Press, Wallingford, Oxfordshire, United Kingdom.

Giacomelli, A., Mancini, M. and Rosso, R., 1997. Integration of ERS 1 PRI imagery and digital terrain models for the assessment of flooded areas. In ESA Proceedings of the 3rd ERS Symposium, Florence, Italy.

Gibbons, J. D. and Chakraborti, S., 2003. Nonparametric Statistical Inference Fourth Edition, Revised and Expanded. Marcel Dekker, New York.

Giorgi, F., 2008. A Simple Equation for Regional Climate Change and Associated Uncertainty. J. Clim., 21, 1589-1604, doi: 10.1175/2007JCLI1763.1.

Giorgi, F. and Mearns, L. O., 2002. Calculation of average, uncertainty range, and reliability of regional climate changes from AOGCM simulations via the "reliability ensemble averaging" (REA) method. J. Clim., 15, 1141-1158.

Giorgi, F. and Mearns, L. O., 2003. Probability of regional climate change based on reliability ensemble averaging (REA) method. Geophys. Res. Lett., 30, 311314, doi: 10.1029/2003GL017130.

Giraldo Osorio, J. D. and García Galiano, S. G., 2011. Building hazard maps of extreme daily rainy events from PDF ensemble, via REA method, on Senegal River Basin. Hydrol. Earth Syst. Sci., 15, 3605-3615.

Giraldo Osorio, J. D and García Galiano, S. G., 2012. Development of a sub-pixel analysis method applied to dynamic monitoring of floods. Int. J. Remote Sens., 33 (7), 2277-2295.

Goswami, B. N., Venugopal, V., Sengupta, D., Madhusoodanan, M.S., and Xavier, P.K., 2006. Increasing Trend of Extreme Rain Events Over India in a Warming Environment. Science, 314, 1442-1445.

Grist, J. P. and Nicholson, S. E., 2001. A study of the dynamic factors influencing the interannual variability of rainfall in the West African Sahel. J. Clim., 14, 13371359. 
Groisman, P. Y., Knight, R. W., Easterling, D. R., Karl, T. R., Hegerl, G. C. and Razuvaev, V. N., 2005. Trends in Intense Precipitation in the Climate Record. J. Climate, 18, 1326-1350.

Herrera, S., Fita, L., Fernández, J. and Gutiérrez, J. M., 2010. Evaluation of the mean and extreme precipitation regimes from ENSEMBLES regional climate multimodel simulations over Spain. J. Geophys. Res., 15, D21117, 13p, doi: 10.1029/2010JD013936

Huntington, T. G., 2006. Evidence for intensification of the global water cycle: review and synthesis. J. Hydrol., 319, 83-95.

IPCC AR4 SYR, 2007. Climate Change 2007: Synthesis Report. Contribution of Working Groups I, II and III to the Fourth Assessment Report of the Intergovernmental Panel on Climate Change., IPCC. Core Writing Team; Pachauri, R. K and Reisinger, A., Ed. ISBN 92-9169-122-4.

IPCC SRES, 2000. Special Report on Emissions Scenarios: A special report of Working Group III of the Intergovernmental Panel on Climate Change. Nakićenović, N., and Swart, R., Ed. Cambridge University Press, ISBN 0-52180081-1, 978-052180081-5.

Jacob, D., 2001. A note to the simulation of the annual and interannual variability of the water budget over the Baltic Sea drainage basin. Meteorol. Atmos. Phys., 77, $61-74$.

Jianghong, S., Zhongming, Z., Qingye, Z. and Yangeng, W., 2004. An algorithm for eliminating the isolated regions based on connected area in image classification. In Proceedings of Geoscience and Remote Sensing Symposium. IEEE International, 5, 3058-3061.

Karambiri, H., García Galiano, S. G., Giraldo, J. D., Yacouba, H., Ibrahim, B., Barbier, B. Polcher, J., 2011. Assessing the impact of climate variability and climate change on runoff in West Africa: the case of Senegal and Nakambe River basins. Atmos. Sci. Lett., AMMA Special Issue, 12, 109-115.

Kendall, M. G., 1975. Rank Correlation Methods. Charles Griffin, London.

Khan, S. I., Hong, Y., Wang, J., Yilmaz, K. K., Gourley, J. J., Adler, R. F., Brakenridge, G. R., Policelli, F., Habib, S. and Irwin, D., 2011. Satellite remote sensing and hydrologic modeling for flood inundation mapping in Lake Victoria Basin: implications for hydrologic prediction in ungauged basins. IEEE Trans. Geosci. Remote Sensing, 49, 85-95. 
Kim, C. and Chi, K., 1998. Flood damage mapping in North Korea using multisensory data. In Proceedings 19th Asian Conference of Remote Sensing, 16-20 November 1998, Manila, Philippines.

Kundzewicz, Z. W., Mata, L. J., Arnell, N. W., Döll, P., Kabat, P., Jiménez, B., Miller, K. A., Oki, T., Sen, Z. and Shiklomanov, I. A., 2007. Freshwater resources and their management. In Climate Change 2007: Impacts, Adaptation and Vulnerability. Contribution of Working Group II to the Fourth Assessment Report of the Intergovernmental Panel on Climate Change, edited by: Parry, M. L., Canziani, O. F., Palutikof, J. P., van der Linden, P. J., and Hanson, C. E., 173210, Cambridge University Press, Cambridge, UK.

Labat, D., Goddéris, Y., Probst, J. L. and Guyot, J. L., 2004. Evidence for global runoff increase related to climate warming. Adv. Water Resour., 27, 631-642.

Lebel, T. and Ali, A., 2009. Recent trends in the Central and Western Sahel rainfall regime (1990-2007). J. Hydrol., 375, 52-64.

Lebel, T., Cappelaere, C., Galle, S., Hanan, N., Kergoat, L., Levis, L., Vieux, B., Descroix, L. Gosset, M., Mougin, E., Peugeot, C. and Seguis, L., 2009. AMMACATCH studies in the Sahelian region of West-Africa: an overview. J. Hydrol., 375, 3-13.

Leduc, C., Favreau, G. and Schroeter, P., 2001. Long term rise in a Sahelian watertable: the continental terminal in South-West Niger. J. Hydrol., 243, 43-54.

Mahé, G. and Paturel, J. E., 2009. 1896-2006 Sahelian annual rainfall variability and runoff increase of Sahelian Rivers. C. R. Geosciences, 341, 538-546.

Mahé, G., Paturel, J. E., Servat, E., Conway, D. and Dezetter, A., 2005. Impact of land use change on soil water holding capacity and river modelling of the Nakambe River in Burkina-Faso. J. Hydrol., 300, 33-43.

Makkonen, L., 2006. Plotting Positions in Extreme Value Analysis. J. Appl. Meteor. Climatol., 45, 334-340.

Maklouf, Z., 1994. Compléments sur le modèle pluie-débit GR4J et essai d'estimation de ses paramètres. PhD thesis, University of Paris XI Orsay, 426.

Martínez, J. M. and Le Toan, T., 2007. Mapping of flood dynamics and spatial distribution of vegetation in the Amazon floodplain using multitemporal SAR data. Remote Sens. Environ., 108, 209-223.

Milly, P. C. D., Betancourt, J., Falkenmark, M., Hirsch, R. M., Kundzewicz, Z. W., Lettenmaier, D. P., and Stouffer, R. J., 2008. Stationarity is dead: Whither water management?. Science, 319, 573-574. 
Murphy, J. M., Sexton, D. M. H., Barnett, D. N., Jones, G. S., Webb, M. J., Collins, M. and Stainforth, D. A., 2004. Quantification of modelling uncertainties in a large ensemble of climate change simulations. Nature, 430, 768-772.

Nash, J. E., Sutcliffe, J. V., 1970. River flow forecasting through conceptual models. Part I - a discussion of principles. J. Hydrol., 10, 282-290.

New, M., Hulme, M. and Jones, P., 2000. Representing twentieth-century space-time climate variability. Part II: Development of 1901-96 monthly grids of terrestrial surface climate. J. Clim., 13, 2217-2238.

Nyborg, L. and Sandholt, I., 2001. NOAA-AVHRR based flood monitoring. In Proceedings of Geoscience and Remote Sensing Symposium. IEEE 2001 International, 4, 1696-1698.

Olson D. M., Dinerstein, E., Wikramanayake, E. D., Burgess, N. D., Powell, G. V .N., Underwood, E. C., D’amico, J. A., Itoua, I., Strand, H. E., Morrison, J. C., Loucks, C. J., Allnutt, T. F., Ricketts, T. H., Kura, Y., Lamoreux, J. F., Wettengel, W. W., Hedao, P. and Kassem, K. R., 2001. Terrestrial Ecoregions of the World: A New Map of Life on Earth. Bioscience, 51 (11), 933-938.

Paeth, H., Born, K., Girmes, R, Podzun, R. and Jacob, D., 2009. Regional climate change in tropical and northern Africa due to greenhouse forcing and land use changes. J. Clim., 22, 114-132.

Paeth, H., Born, K., Podzun, R. and Jacob, D., 2005. Regional dynamical downscaling over West Africa: model evaluation and comparison of wet and dry years. Meteorol. Z., 14, 349-367.

Paeth, H., Hall, N. M. J., Gaertner, M., Alonso, M. D., Moumouni, S., Polcher, J., Ruti, P. M., Fink, A. H., Gosset, M., Lebel, T., Gaye, A. T., Rowell, D. P., MoufoumaOkia, W., Jacob, D., Rockell, B., Giorgi, F. and Rummukainen, M., 2011. Progress in regional downscaling of West African precipitation. Atmos. Sci. Let., $12,75-82$.

Perkins, S. E., Pitman, A. J., Holbrook, N. J. and McAneney, J., 2007. Evaluation of the AR4 climate models' simulated daily maximum temperature, minimum temperature, and precipitation over Australia using probability density functions. J. Clim., 20, 4356-4376.

Polcher, J., Parker, D. J., Gaye, A., Diedhiou, A., Eymard, L., Fierli, F., Genesio, L., Höller, H., Janicot, S., Lafore, J. P., Karambiri, H., Lebel, T., Redelsperger, J. L., Reeves, C. E., Ruti, P., Sandholt, I. and Thorncroft, C., 2011. AMMA's 
contribution to the evolution of prediction and decision-making systems for West Africa. Atmos. Sci. Lett., 12, 2-6, doi: 10.1002/asl.320.

Profeti, G. and Macintosh, H., 1997. Flood management through Landsat TM and ERS SAR data: a case study. Hydrol. Process., 11, 1397-1408.

Räisänen, J. and Palmer, T. N., 2001. A probability and decision-model analysis of a multimodel ensemble of climate change simulations. J. Climate, 14, 3212-3226.

Rajeevan, M., Bhate, J., and Jaswal, A. K., 2008. Analysis of variability and trends of extreme rainfall events over India using 104 years of gridded daily rainfall data. Geophys. Res. Lett., 35, L18707, doi: 10.1029/2008GL035143.

Rigby, R. A. and Stasinopoulos, D. M., 2005. Generalized additive models for location scale and shape. Appl. Stat., 54, 507-554.

Rummukainen, M., 2010. State-of-the-art with regional climate models. WIREs Climate Change. 1, 82-96. doi: 10.1002/wcc.8

Ruti, P. M., Williams, J. E., Hourdin, F., Guichard, F., Boone, A., Van Velthoven, P., Favot, F., Musat, I., Rummukainen, M., Dom’ınguez, M., Gaertner, M. A' ., Lafore, J. P., Losada, T., Rodriguez de Fonseca, M. B., Polcher, J., Giorgi, F., Xue, Y., Bouarar, I., Law, K., Josse, B., Barret, B., Yang, X. and Mari, C., 2011. The West African climate system: a review of the AMMA model intercomparison initiatives. Atmos. Sci. Let., 12, 116-122, doi: 10.1002/asl.305.

Saich, P., Thompson, J. and Rebelo, L., 2001. Monitoring wetland extent and dynamics in the Cat Tien National Park, Vietnam, using space-based radar remote sensing. In Geoscience and Remote Sensing Symposium. IEEE 2001 International, 7, 3099-3101.

Sakamoto, T., van Nguyen, N. Ohno, H., Ishitsuka, N. and Yokozawa, M., 2005. Spatio-temporal distribution of rice phenology and cropping systems in the Mekong Delta with special reference to the seasonal water flow of the Mekong and Bassac rivers. Remote Sens. Environ., 100, 1-16.

Sánchez, E., Domínguez, M., Romera, R., López de la Franca, N., Gaertner, M. A., Gallardo, C. and Castro, M., 2011. Regional modeling of dry spells over the Iberian Peninsula for present climate and climate change conditions: A letter. Clim. Change, 10, 625-634.

Sánchez, E., Romera, R., Gaertner, M. A., Gallardo, C. and Castro, M., 2009. A weighting proposal for an ensemble of regional climate models over Europe driven by 1961-2000 ERA40 based on monthly precipitation probability density functions. Atmos. Sci. Lett., 10, 241-248. 
Sanyal, J. and Lu, X., 2004. Application of remote sensing in flood management with special reference to monsoon Asia: a review. Nat. Hazards, 33, 283-301.

Sanyal, J. and Lu., X., 2005. Remote sensing and GIS-based flood vulnerability assessment of human settlements: a case study of Gangetic West Bengal, India. Hydrol. Process., 19, 3699-3716.

Séguis, L., Boulain, N., Cappelaere, B., Cohard, J. M., Favreau, G., Galle, S., Guyot, A., Hiernaux, P., Mougin, E., Peugeot, C., Ramier, D., Seghieri, J., Timouk, F., Demarez, V., Demarty, J., Descroix, L., Descloitres, M., Grippa, M., Guichard, F., Kamagate, B. S., Kergoat, L., Lebel, T., Le Dantec, V., Le Lay, M., Massuel, S. and Trichon, V., 2011. Contrasted land surface processes along the West African rainfall gradient. Atmos. Sci. Lett., 12, 31-37, doi: 10.1002/asl.327.

Smith, L., 1997. Satellite remote sensing of river inundation area, stage and discharge: a review. Hydrol. Process., 11, 1427-1439.

Stasinopoulos, D. M. and Rigby, R. A., 2007. Generalized additive models for location scale and shape (GAMLSS) in R. J. Stat. Software, 23, 1-46.

Stasinopoulos, D. M., Rigby, R. A. and Akantziliotou, C., 2008. Instructions on how to use the gamlss package in $R$, Second Edition.

Sylla, M. B., Dell'Aquilla, A., Ruti, P. M. and Giorgi, F., 2010a. Simulation of the intraseasonal and the interannual variability of rainfall over West Africa with RegCM3 during the monsoon period. Int. J. Climatol., 1865-1883.

Sylla, M. B., Coppola, E., Mariotti, L., Giorgi, F., Ruti, P. M., Dell'Aquila, A. and Bi, X., 2010b. Multiyear simulation of the African climate using a regional climate model (RegCM3) with the high resolution ERA-interim reanalysis. Clim. Dyn., $35,231-247$.

Verhoeye, J. and De Wulf, R., 2002. Land cover mapping at sub-pixel scales using linear optimization techniques. Remote Sens. Environ., 79, pp. 96-104.

Tapiador, F. J., Sánchez, E. and Romera, R., 2009. Exploiting an ensemble of regional climate models to provide robust estimates of projected changes in monthly temperature and precipitation probability distribution functions. Tellus, 61A, 5771, doi: 10.1111/j.1600-0870.2008.00374.x.

Tebaldi, C. and Knutti, R., 2007. The use of the multi-model ensemble in probabilistic climate projections. Philos. Trans. R. Soc. A-Math. Phys. Eng. Sci., 365, 20532075, doi: 10.1098/rsta.2007.2076. 
Tebaldi, C., Smith, R. L., Nychka, D. and Mearns, L. O., 2005. Quantifying Uncertainty in Projections of Regional Climate Change: A Bayesian Approach to the Analysis of Multimodel Ensembles. J. Clim., 18, 1524-1540.

Towsend, P. A. and Walsh, S. J., 1998. Modeling floodplain inundation using an integrated GIS with radar and optical remote sensing. Geomorphology, 21, 295-312.

Töyráä, J. and Pietroniro, A., 2005. Towards operational monitoring of a northern wetland using geomatics-based techniques. Remote Sens. Environ., 97, pp. 174-191.

Tralli, D. M., Blom, R. G., Zlotnicki, V., Donneallan, A. and Evans, D. L., 2005. Satellite remote sensing of earthquake, volcano, flood, landslide and coastal inundation hazards. ISPRS-J. Photogramm. Remote Sens., 59, 185-198.

van Buuren, S. and Fredriks, M., 2001. Worm plot: a simple diagnostic device for modelling growth reference curves. Statist. Med., 20, 1259-1277.

Villarini, G., Smith, J. A., and Napolitano, F., 2010. Nonstationary modelling of a long record of rainfall and temperature over Rome. Adv. Water Res., 33, 1256-1267.

Wang, Y., 2002. Mapping extent of floods: what we have learned and how we can do better. Nat. Hazards Rev., 3, 68-73.

Wang, Y., 2004. Using Landsat 7 TM data acquired days after a flood event to delineate the maximum flood extent on a coastal floodplain. Int. J. Remote Sens., 25, 959-974.

Wang, Y., Colby, J. D. and Mulcahy, K. A., 2002. An efficient method for mapping flood extent in a coastal floodplain using Landsat TM and DEM data. Int. J. Remote Sens., 23, 3681-3696.

Wigley, T. M. L. and Raper, C. B., 2001. Interpretation of high projections for globalmean warming. Science, 293, 451-454.

Xu, C. Y. and Singh V. P., 2002. Cross comparison of empirical equations for calculating potential evapotranspiration with data from Switzerland. Water Resour. Res., 16, 197-219.

Yang, C., Zhou, C. and Wan, Q., 1999. Deciding the flood extent with Radarsat SAR data and Image Fusion. In Proceedings of 20th Asian Conference of Remote Sensing, 22-25 November 1999, Hong Kong.

Zhan, X., Defries, R., Townshend, J., Dimiceli, C., Hansen, M., Huang, C. and Sohlberg, R., 2000. The $250 \mathrm{~m}$ global land cover change product from the 
moderate resolution imaging spectroradiometer of NASA's Earth Observation System. Int. J. Remote Sens., 21, 1433-1460.

Zhan, X., Sohlberg, R., Townshend, J., Dimiceli, C., Carroll, M., Eatsman, J., Hansen, M. and Defries, R., 2002. Detection of land cover changes using MODIS $250 \mathrm{~m}$ data. Remote Sens. Environ., 83, 336-350. 


\section{APÉNDICE 1}

Índice de impacto de las publicaciones incluidas en la Tesis, o justificación documentada de la importancia científica de los canales de publicación utilizados.

\section{A.1.1 Índice de Impacto de las Publicaciones}

\section{- $\quad$ Artículo 1}

Referencia:

García Galiano, S. G. and Giraldo Osorio, J. D., 2010. Analysis of impacts on hydrometeorological extremes in the Senegal river basin from REMO RCM. The 2nd Lund Regional-scale Climate Modelling Workshop: Part II. Meteorologische Zeitschrift, 19 (4), 375-384.

Factor de impacto:

$1.402(\mathrm{Q})$.

Fuente: ISI Web of Knowledge, Journal Citation Reports, 2010 JCR Science Edition.

\section{- $\quad$ Artículo 2}

Referencia:

Karambiri, H., García Galiano, S. G., Giraldo, J. D., Yacouba, H., Ibrahim, B., Barbier, B. Polcher, J., 2011. Assessing the impact of climate variability and climate change on runoff in West Africa: the case of Senegal and Nakambe River basins, Atmospheric Science Letters. AMMA Special Issue, 12: 109-115.

Factor de impacto:

$1.433(\mathrm{Q} 3)$.

Fuente: ISI Web of Knowledge, Journal Citation Reports, 2010 JCR Science Edition.

\section{- $\quad$ Artículo 3}

Referencia:

Giraldo Osorio, J. D. and García Galiano, S. G., 2011. Building hazard maps of extreme daily rainy events from PDF ensemble, via REA method, on Senegal River Basin. Hydrol. Earth Syst. Sci., 15, 3605-3615.

Factor de impacto:

$2.463(\mathrm{Q} 1)$.

Fuente: ISI Web of Knowledge, Journal Citation Reports, 2010 JCR Science Edition. 


\section{- $\quad$ Artículo 4}

Referencia:

Giraldo Osorio, J. D, García Galiano, S. G., 2012. Development of a sub-pixel analysis method applied to dynamic monitoring of floods, International Journal of Remote Sensing, 33 (7), 2277-2295.

Factor de impacto:

$1.182(\mathrm{Q} 2)$.

Fuente: ISI Web of Knowledge, Journal Citation Reports, 2010 JCR Science Edition.

\section{A.1.2 Importancia Científica del Capítulo de Libro Internacional}

Referencia:

Garcia Galiano, S. G., Giraldo Osorio, J. D., 2011. Non stationary analysis of spatial patterns of extreme rainfall events in West Africa, In: Hydro-climatology: variability and change (Eds. S.W. Franks, E. Boegh, E. Blyth, D.M. Hannah, K.K. Yilmaz). IAHS-AISH Publication, 344, 75-81. IAHS Press, Wallingford, Oxfordshire, United Kingdom.

Congreso de alto nivel internacional. Por lo que ha sido presentado de forma oral en la 25th International Union of Geodesy and Geophysics General Assembly, IUGG 2011 (Melbourne, Australia).

Fuente: Indexado en SCOPUS. 


\section{ISI Web of Knowledge}

Journal Citation Reports ${ }^{\circledR}$

\section{Ain WELCOME ? HELP R REURRM TO \\ 2010 JCR Science Edition \\ Rank in Category: METEOROLOGISCHE ZEITSCHRIFT}

\section{Journal Ranking $\mathrm{i}$}

For 2010, the journal METEOROLOGISCHE ZEITSCHRIFT has an Impact Factor of $\mathbf{1 . 4 0 2 .}$

This table shows the ranking of this journal in its subject categories based on Impact Factor.

\begin{tabular}{|c|c|c|c|}
\hline Category Name & $\begin{array}{c}\text { Total Journals } \\
\text { in Category }\end{array}$ & $\begin{array}{c}\text { Journal Rank } \\
\text { in Category }\end{array}$ & $\begin{array}{c}\text { Quartile } \\
\text { in Category }\end{array}$ \\
\hline METEOROLOGY \& ATMOSPHERIC SCIENCES & 68 & 41 & $\mathrm{Q} 3$ \\
\hline
\end{tabular}

\section{Category Box Plot i}

For 2010, the journal METEOROLOGISCHE ZEITSCHRIFT has an Impact Factor of $\mathbf{1 . 4 0 2 .}$

This is a box plot of the subject category or categories to which the journal has been assigned. It provides information about the distribution of journals based on Impact Factor values. It shows median, 25th and 75th percentiles, and the extreme values of the distribution.

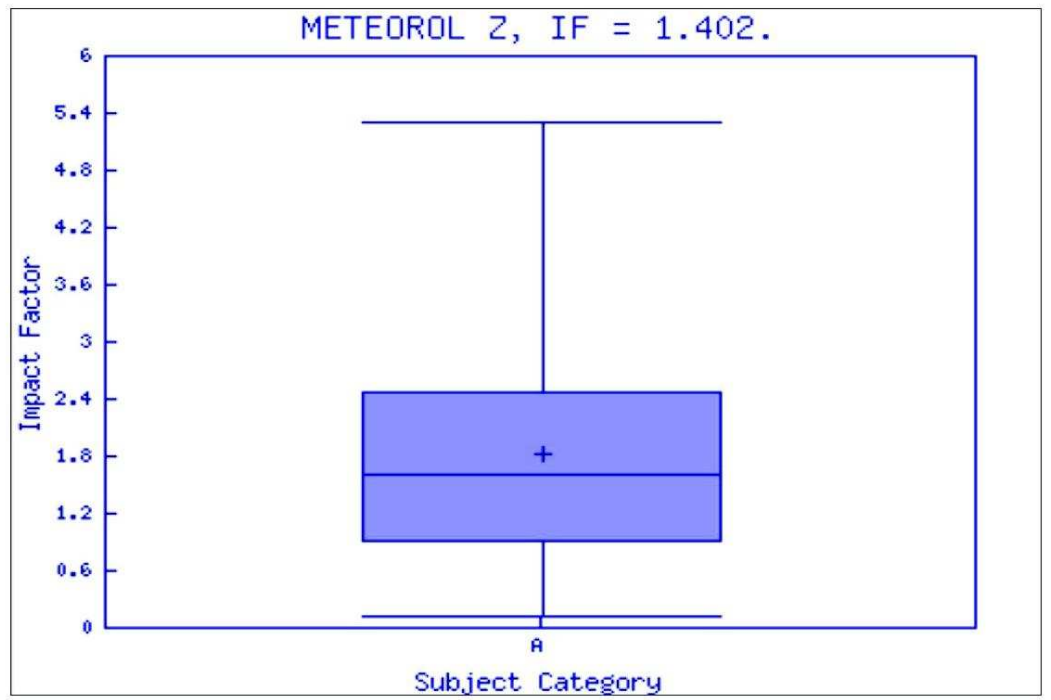

Key METEOROLOGY \& 8 ATMOSPHERIC SCIENCES 



\section{ISI Web of Knowledge}

Journal Citation Reports

\begin{tabular}{|c|c|c|}
\hline Welcome & ? HELP & 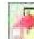 \\
\hline
\end{tabular}

2010 JCR Science Edition

\section{Rank in Category: Atmospheric Science Letters}

\section{Journal Ranking $\mathrm{i}$}

For 2010, the journal Atmospheric Science Letters has an Impact Factor of 1.433.

This table shows the ranking of this journal in its subject categories based on Impact Factor.

\begin{tabular}{|c|c|c|c|}
\hline Category Name & $\begin{array}{c}\text { Total Journals } \\
\text { in Category }\end{array}$ & $\begin{array}{c}\text { Journal Rank } \\
\text { in Category }\end{array}$ & $\begin{array}{c}\text { Quartile } \\
\text { in Category }\end{array}$ \\
\hline METEOROLOGY \& ATMOSPHERIC SCIENCES & 68 & 40 & $\mathrm{Q3}$ \\
\hline
\end{tabular}

\section{Category Box Plot i)}

For 2010, the journal Atmospheric Science Letters has an Impact Factor of $\mathbf{1 . 4 3 3 .}$

This is a box plot of the subject category or categories to which the journal has been assigned. It provides information about the distribution of journals based on Impact Factor values. It shows median, 25th and 75th percentiles, and the extreme values of the distribution.

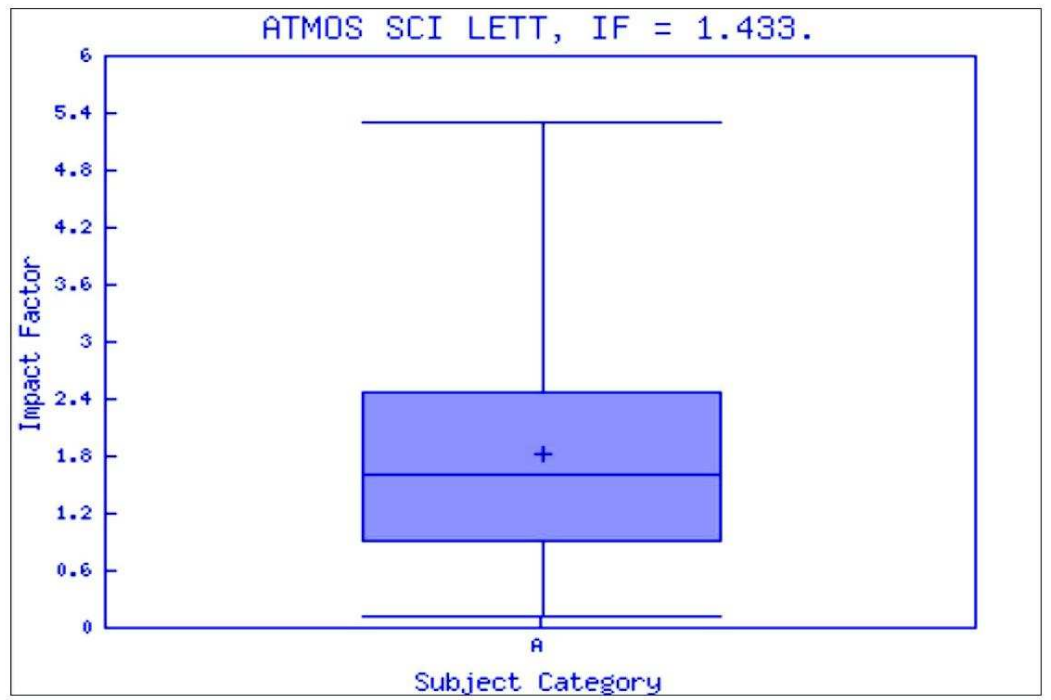

Key METEOROLOGY ATMOSPHERIC SCIENCES 



\section{ISI Web of Knowledge}

Journal Citation Reports ${ }^{\oplus}$

WELCOME ? HELP 7 RETURN TO

2010 JCR Science Edition

\section{Rank in Category: HYDROLOGY AND EARTH SYSTEM SCIENCES}

\section{Journal Ranking $\mathrm{D}$}

For 2010, the journal HYDROLOGY AND EARTH SYSTEM SCIENCES has an Impact Factor of $\mathbf{2 . 4 6 3}$

This table shows the ranking of this journal in its subject categories based on Impact Factor.

\begin{tabular}{|l|c|c|c|}
\hline \multicolumn{1}{|c|}{ Category Name } & $\begin{array}{c}\text { Total Journals } \\
\text { in Category }\end{array}$ & $\begin{array}{c}\text { Journal Rank } \\
\text { in Category }\end{array}$ & $\begin{array}{c}\text { Quartile } \\
\text { in Category }\end{array}$ \\
\hline GEOSCIENCES, MULTIDISCIPLINARY & 167 & 31 & $\mathrm{Q} 1$ \\
\hline WATER RESOURCES & 76 & 5 & $\mathrm{Q1}$ \\
\hline
\end{tabular}

Category Box Plot D

For 2010, the journal HYDROLOGY AND EARTH SYSTEM SCIENCES has an Impact Factor of $\mathbf{2 . 4 6 3 .}$

This is a box plot of the subject category or categories to which the journal has been assigned. It provides information about the distribution of journals based on Impact Factor values. It shows median, 25 th and 75 th percentiles, and the extreme values of the distribution.

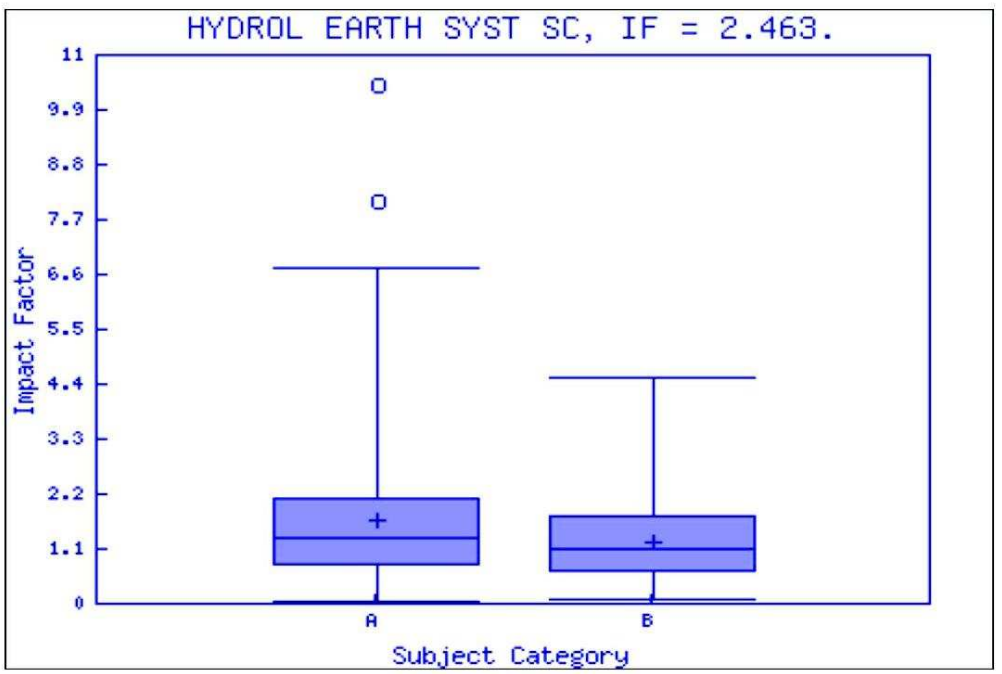

Key

GEOSCIENCES,

MULTIDISCIPLINARY

B - WATER RESOURCES 



\section{ISI Web of Knowledge}

Journal Citation Reports

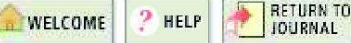 \\ 2010 JCR Science Edition \\ Rank in Category: INTERNATIONAL JOURNAL OF REMOTE SENSING}

\section{Journal Ranking i}

For 2010, the journal INTERNATIONAL JOURNAL OF REMOTE SENSING has an Impact Factor of 1.182.

This table shows the ranking of this journal in its subject categories based on Impact Factor.

\begin{tabular}{|l|c|c|c|}
\hline \multicolumn{1}{|c|}{ Category Name } & $\begin{array}{c}\text { Total Journals } \\
\text { in Category }\end{array}$ & $\begin{array}{c}\text { Journal Rank } \\
\text { in Category }\end{array}$ & $\begin{array}{c}\text { Quartile } \\
\text { in Category }\end{array}$ \\
\hline IMAGING SCIENCE \& PHOTOGRAPHIC TECHNOLOGY & 19 & 5 & $\mathrm{Q} 2$ \\
\hline REMOTE SENSING & 23 & 9 & $\mathrm{Q} 2$ \\
\hline
\end{tabular}

Category Box Plot i)

For 2010, the journal INTERNATIONAL JOURNAL OF REMOTE SENSING has an Impact Factor of 1.182.

This is a box plot of the subject category or categories to which the journal has been assigned. It provides information about the distribution of journals based on Impact Factor values. It shows median, 25th and 75th percentiles, and the extreme values of the distribution.

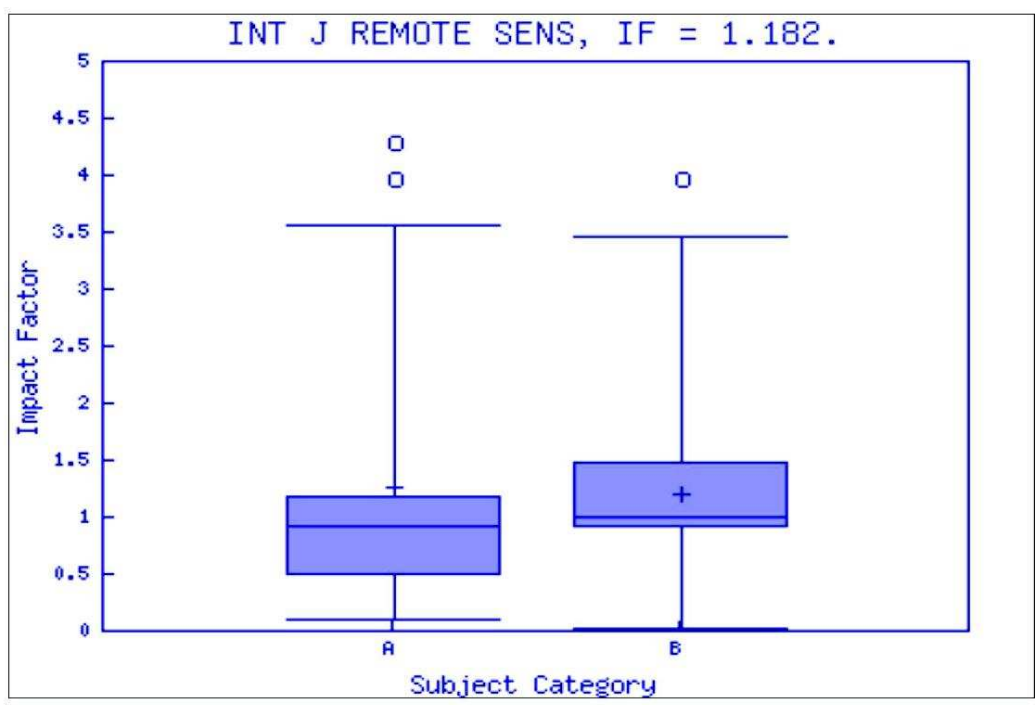

Acceptable Use Policy

Copyright (c) 2011 Thomson Reuters.
Key

IMAGING

SCIENCE \&

PHOTOGRAPHIC

TECHNOLOGY

B - REMOTE 



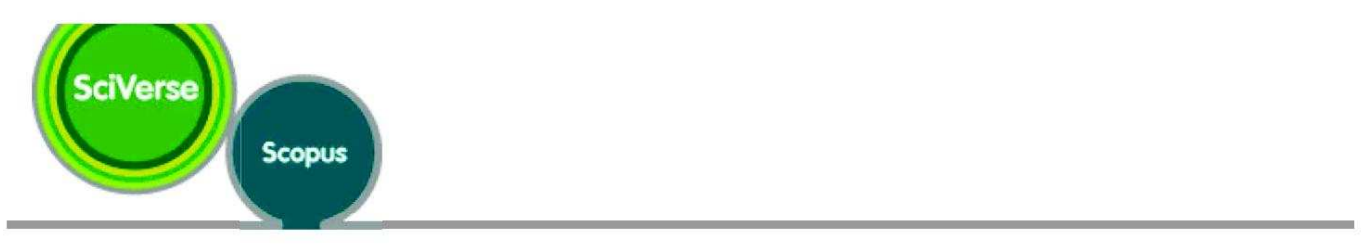

\section{Documents}

Galiano, S.G.G., Osorio, J.D.G.

Non-stationary analys is of spatial patterns of extreme rainfall events in West Africa (2011) IAHS-AISH Publication, 344, pp. 75-81.

Technical University of Cartagena, R and D Group of Water Resources Management, Department of Civil Engineering, Paseo Alfonso XIII, 52, 30203, Cartagena, Spain

\section{Abstract}

Heavy storm events frequently cause extensive damage, and often result in loss of life and property. The objective of this work is to build maps of Annual Maximum Daily Rainfall (AMDR) for various return periods for the Senegal River Basin. However, traditional stationary analyses are not suitable, since meaningful trends have been detected in historical hydrometeorological time series. Therefore, the GAMLSS (Generalized Additive Models for Location, Scale and Shape) tool is applied to fit the parameters of the probability density functions (pdfs). AMDR time series were estimated using observed daily rainfall grids and regional climate models (RCMs). The wide divergence in predicted trends from RCMs imposes the use of ensemble pdfs, which can be built using bootstrapping techniques. The plausible AMDR maps associated with various quantiles, interpolated from these ensemble pdfs, could be used by stakeholders to develop strategies of mitigation and adaptation to climate change impacts on floods events. (C) 2011 IAHS Press.

\section{Author Keywords}

Bootstrapping; Ensemble probability density function; GAMLSS; Maximum daily rainfall; Non-stationarity; Regional climate models

\section{Correspondence Address}

Galiano S.G.G.; Technical University of Cartagena, R and D Group of Water Resources Management, Department of Civil Engineering, Paseo Alfonso XIII, 52, 30203, Cartagena, Spain; email: sandra.garcia@upct.es

Sponsors: International Association of Hydrological Sciences (IAHS); ICCLAS; ICSW; HYDROMET; Int. Assoc. Meteorol. Atmos. Sci. (IAMAS)

Conference name: Hydro-climatology - Variability and Change Symposium, part of the 25th International Union of Geodesy and Geophysics General Assembly, IUGG 2011

Conference date: 28 June 2011 through 7 July 2011

Conference location: Melbourne, VIC

Conference code: 87439

ISSN: 01447815

ISBN: 9781907161193

CODEN: IAPUE

Language of Original Document: English

Abbreviated Source Title: IAHS-AISH Publ.

Document Type: Conference Paper

Source: Scopus

About Scopus

What is Scopus

Content coverage

What do users think

Latest

Tutorials
Contact and Support

Contact and support

Live Chat

About Elsevier

About Elsevier

About Sciverse

About Scival

Terms and Conditions

Privacy Policy

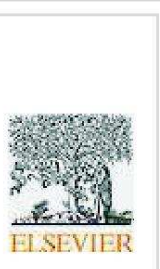





\section{APÉNDICE 2}

Alcance de las patentes y justificación documentada de su importancia y vinculación con la Tesis desarrollada.

\section{A.2.1 Registro de Propiedad Intelectual 1}

\section{- Referencia}

Giraldo Osorio, J. D. y García Galiano, S. G., 2011. Subpixels Toolbox. Técnica de downscaling de imágenes satélite. Seguimiento dinámico de avenidas en grandes extensiones. Comandos GRASS desarrollados: i.subpixel, i.dod, i.isolate. UPCT RPI 08/2011/173.

\section{- Importancia}

- Desarrollo de una herramienta que facilite el análisis de sub-píxeles para la detección de zonas inundadas.

- Integración de los Modelos de Elevación Digital (DEM) en el análisis de inundaciones.

- Codificación de los comandos necesarios, con lenguajes de programación y SIG con licencias GNU que permitan que la metodología de clasificación sea aplicable por los gestores y responsables de las decisiones en países en vías de desarrollo.

- Contribución al desarrollo de estrategias de adaptación al cambio y variabilidad climática frente a inundaciones.

Este software permitió cumplir con los objetivos propuestos en los siguientes artículos, donde se puede verificar el alcance e importancia de la creación de Spells Toolbox:

- Giraldo Osorio, J. D, García Galiano, S. G., 2012. Development of a sub-pixel analysis method applied to dynamic monitoring of floods, International Journal of Remote Sensing, 33 (7), 2277-2295.

\section{A.2.2 Registro de Propiedad Intelectual 2}

\section{- Referencia}

Giraldo Osorio, J. D. y García Galiano, S. G., 2011. Spells Toolbox. Análisis de rachas en series de mapas hidrológicos. Comandos GRASS desarrollados: r.series.spell, r.series.spell.point. UPCT RPI 08/2011/176. 


\section{- Importancia}

- Desarrollo de una herramienta que facilite el análisis de rachas a partir de mapas digitales de variables hidrológicas.

- Construcción de nuevos mapas que describen las características de las rachas, o permiten obtener series puntuales de las mismas.

- Codificación de los comandos necesarios, con lenguajes de programación y SIG con licencias GNU-GPL que permitan que la metodología de clasificación sea aplicable por los gestores y responsables de las decisiones en países en vías de desarrollo.

- Contribución al desarrollo de estrategias de adaptación al cambio y variabilidad climática frente a eventos extremos (sequías, rachas húmedas, rachas de temperaturas máximas, etc.).

Se debe destacar que el software Spells Toolbox, se encuentra entre la transferencia tecnológica de la UPCT a la Spin Off FutureWater S.L. en fase de constitución (aprobada en Reunión de Consejo de Gobierno del 22 de diciembre de 2011).

Este software permitió cumplir con los objetivos propuestos en los siguientes artículos, donde se puede verificar el alcance e importancia de la creación de Spells Toolbox:

- García Galiano, S. G. and Giraldo Osorio, J. D., 2010. Analysis of impacts on hydrometeorological extremes in the Senegal river basin from REMO RCM. Meteorologische Zeitschrift, 19 (4), 375-384.

- Karambiri, H., García Galiano, S. G., Giraldo, J. D., Yacouba, H., Ibrahim, B., Barbier, B. Polcher, J., 2011. Assessing the impact of climate variability and climate change on runoff in West Africa: the case of Senegal and Nakambe River basins, Atmospheric Science Letters. AMMA Special Issue, 12: 109-115.

\section{A.2.3 Registro de Propiedad Intelectual 3}

\section{- Referencia}

Giraldo Osorio, J. D. y García Galiano, S. G., 2011. Series Toolbox. Análisis de series de mapas hidrológicos con variables hidrológicas. Comandos GRASS desarrollados: r.series.new, r.series.new.point. UPCT RPI 08/2011/177.

\section{- Importancia}

- Desarrollo de una herramienta que facilite el análisis de series temporales de mapas digitales de variables hidrológicas. 
- Construcción de nuevos mapas que describen las características estadísticas de la variable hidrológica de interés, o permite obtener series puntuales de la variable.

- Codificación de los comandos necesarios, con lenguajes de programación y SIG con licencias GNU-GPL que permitan que la metodología de clasificación sea aplicable por los gestores y responsables de las decisiones en países en vías de desarrollo.

- Contribución al desarrollo de estrategias de adaptación al cambio y variabilidad climática de patrones espacio-temporales de estadísticos (medias, desvíos, etc.) de variables hidrometeorológicas.

Se debe destacar que el software Series Toolbox, se encuentra entre la transferencia tecnológica de la UPCT a la Spin Off FutureWater S.L. en fase de constitución (aprobada en Reunión de Consejo de Gobierno del 22 de diciembre de 2011).

Este software permitió cumplir con los objetivos propuestos en los siguientes artículos, donde se puede verificar el alcance e importancia de la creación de Series Toolbox:

- Giraldo Osorio, J. D. and García Galiano, S. G., 2011. Building hazard maps of extreme daily rainy events from PDF ensemble, via REA method, on Senegal River Basin. Hydrol. Earth Syst. Sci., 15, 3605-3615.

- Garcia Galiano, S. G., Giraldo Osorio, J. D., 2011. Non stationary analysis of spatial patterns of extreme rainfall events in West Africa. In: Hydro-climatology: variability and change. (Eds. S.W. Franks, E. Boegh, E. Blyth, D.M. Hannah, K.K. Yilmaz). IAHS Publ. 344, IAHS Press, Wallingford, Oxfordshire, United Kingdom. 



\section{APÉNDICE 3}

Técnicas de Bootstrapping

\section{A.3.1 Fundamentos}

Fox (2002) presenta una definición matemática simple del bootstrapping no paramétrico. Supóngase que se tiene una muestra $\mathbf{S}=\left\{X_{1}, X_{2}, \ldots, X_{n}\right\}$ que proviene de una población $\mathbf{P}=\left\{x_{1}, x_{2}, \ldots, x_{N}\right\}$ (aunque $\mathbf{P}$ podría ser definida por una PDF, y de esta manera ser una población infinita). Por ahora, supóngase que $N>>n$.

Ahora, se considerará que se está interesado en algún estadístico $T=t(\mathbf{S})$, el cual es un estimado del parámetro de la población $\theta=t(\mathbf{P})$. La estadística inferencial hará conjeturas sobre la estructura de la población, y a partir de ésta se derivará la distribución muestral de $T$. Esta aproximación puede tener importantes deficiencias. En contraste, el bootstrap no paramétrico permite estimar la distribución muestral del estadístico de forma empírica, sin hipótesis sobre la población y sin derivar la distribución muestral de forma explícita.

La idea clave del bootstrap no paramétrico es que se obtendrá una muestra de tamaño $n$ de entre los elementos de $\mathbf{S}$, muestreando con reemplazo. Llámese la muestra boostrap $\mathbf{S}_{1}{ }^{*}=\left\{X_{11}{ }^{*}, X_{12}{ }^{*}, \ldots, X_{1 n}{ }^{*}\right\}$. Es necesario el muestreo con reemplazo, porque de otra forma se estaría simplemente reproduciendo la muestra original $\mathbf{S}$. En efecto, se está tratando la muestra $\mathbf{S}$ como un estimado de la población $\mathbf{P}$. Esto es, cada elemento $X_{i}$ de $\mathbf{S}$ es seleccionado para la muestra bootstrap con probabilidad $1 / n$, imitando la elección original de la muestra $\mathbf{S}$ de la población $\mathbf{P}$. El procedimiento de extraer muestras bootstrap se repite muchas veces. La muestra bootstrap $b^{\text {th }}$ sería entonces $\mathbf{S}_{\mathbf{b}}{ }^{*}=\left\{X_{b 1}{ }^{*}, X_{b 2}{ }^{*}, \ldots, X_{b n}{ }^{*}\right\}$.

Ahora, calcúlese el estadístico $T$ para cada una de las muestras bootstrap, lo que equivale a $T_{b}{ }^{*}=t\left(\mathbf{S}_{\mathrm{b}}{ }^{*}\right)$. Entonces, la distribución de $T_{b} *$ alrededor del estimado original de $T$ es análogo a la distribución muestral del estimador $T$ alrededor del parámetro poblacional $\theta$. Por ejemplo, el promedio de los estadísticos bootstraped (muestreados con reemplazo), sería

$\bar{T}^{*}=\hat{E}^{*}\left(T^{*}\right)=\frac{\sum_{b=1}^{R} T_{b} *}{R}$ 
Desde la ecuación anterior se estima el valor esperado de los estadísticos bootstraped; entonces

$\bar{B}^{*}=\overline{T^{*}}-T$

es un estimado del sesgo de $T$, esto es $T-\theta$. De forma similar, la varianza bootstrap estimada de $T^{*}$,

$\hat{V^{*}}\left(T^{*}\right)=\frac{\sum_{b=1}^{R}\left(T_{b} *-\bar{T} *\right)}{R-1}$

estima la varianza muestral de $T$.

\section{A.3.2 Intervalos de Confianza Bootstrap}

Los intervalos de confianza pueden ser construidos de varias formas. La teoría normal para los intervalos, supone que el estadístico $T$ se distribuye normalmente (que es, con frecuencia, una aproximación correcta para estadísticos calculados con muestras suficientemente grandes), el intervalo de confianza para $100(1-\alpha) \%$ tiene la siguiente forma:

$\theta=\left(T-\hat{B}^{*}\right) \pm Z_{1-\alpha / 2} \hat{S E}^{*}\left(T^{*}\right)$

donde

$$
\hat{S E}^{*}\left(T^{*}\right)=\sqrt{\hat{V^{*}}\left(T^{*}\right)}
$$

es el estimado bootstrap del error estándar de $T, Z_{1-\alpha / 2}$ es el cuantil $1-\alpha / 2$ de la distribución normal estándar (e. g. para construir el intervalo de confianza al 95\%, donde $\alpha=0.05$, entonces $Z_{1-\alpha 2}=1.96$ ).

Una aproximación alternativa son los intervalos de cuantiles bootstrap. Consisten en utilizar los cuantiles empíricos de $T_{b}$ * para construir el intervalo de confianza de $\theta$. Se han construido $R$ muestras bootstrap del estadístico $T . T_{(1)} *, T_{(2)} * \ldots, T_{(R)} *$ son las réplicas bootstrap ordenadas del estadístico. Entonces el intervalo de confianza es

$$
T_{[(R+1) \alpha / 2]}<\theta<T_{[(R+1)(1-\alpha / 2)]}
$$


donde los corchetes indican el redondeo al entero más cercano. Por ejemplo, si $R=$ 1000 y $\alpha=0.05$, entonces el intervalo de confianza será $T_{25}<\theta<T_{975}$. Este último procedimiento es el utilizado en esta Tesis. 



\section{APÉNDICE 4}

Acrónimos y siglas utilizadas en este documento.

2iE: Institut International de l'Ingénierie de l'Eau et de l'Environnement, Burkina Faso.

AEJ: African Easterly Jet.

AEW: African Easterly Waves (or Tropical Easterly Waves).

AMDR: Annual Maximum Daily Rainfall.

AMDSL: Annual Maximum dry Spell Length.

AMMA: African Monsoon Multidisciplinary Analyses.

CDF: Cumulative Distribution Function.

CRU: Climate Research Unit.

DEM: Digital Elevation Model.

DJF: December - January - February (invierno boreal).

DOD: Drainage on DEM.

DSL: Dry Spell Length.

ENSO: El Niño - Southern Oscillation.

ECMWF: European Centre for Medium-Range Weather Forecasts.

ETM+: Enhanced Thematic Mapper Plus.

GAMLSS: Generalized Additive Models for Location, Scale and Shape.

GCM: General Circulation Model.

GFDL: Geophysical Fluid Dynamics Laboratory.

GFS: Guinean Forest Savanna (ecorregión).

GHGE: Greenhouse Gases Emission.

GNU-GPL: GNU General Public License.

GRASS: Geographic Resources Analysis Support System.

$\mathrm{ICI}$ : Isolated Cluster Identification.

IPCC: Intergovernmental Panel on Climate Change.

IRD: Institut de Recherche pour le Développement, Francia.

ITZC: Intertropical Convergence Zone.

JJA: June - July - August (verano boreal).

LMM: Linear Mixture Model.

MCS: Mesoscale Convective System. 
MODIS: Moderate Resolution Imaging Spectroradiometer.

NGA: National Geospatial-Intelligence Agency.

NASA: National Aeronautics and Space Administration.

OMVS: Organization pour la Mise en Valeur du fleuve Sénégal.

NAO: North Atlantic Oscillation.

PDF: Probability Density Function.

PPE: Perturbed Physics Ensemble.

REA: Reliability Ensemble Average.

RCM: Regional Climate Model.

SA: Sub-pixels Analysis.

SAS: Sahelian Acacia Savanna (ecorregión).

SBC: Schwarz Bayesian criterion.

SCA: Spatial Coherence Analysis.

SIG: Sistema de Información Geográfica.

SRES: Special Report Emission Scenarios.

SRTM: Shuttle Radar Topography Mission.

SST: Sea Surface Temperature.

TSSK: Two Sample Smirnov-Kolmogorov.

UNEP: United Nations Environment Programme.

UPCT: Universidad Politécnica de Cartagena, España.

WAM: West African Monsoon.

WMO: World Meteorological Organization.

WSS: West Sudanian Savanna (ecorregión). 